\title{
E.E. Evans-Pritchard
}

\author{
(1963)
}

\section{La femme \\ dans les sociétés primitives et autres essais d'anthropologie sociale}

\author{
Un document produit en version numérique par Jean-Marie Tremblay, bénévole, \\ professeur de sociologie au Cégep de Chicoutimi \\ Courriel:jmt_sociologue@,videotron.ca \\ Site web: http://www.uqac.ca/jmt-sociologue/ \\ Dans le cadre de la collection: "Les classiques des sciences sociales" \\ Site web: http://www.uqac.ca//Classiques_des_sciences_sociales/ \\ Une collection développée en collaboration avec la Bibliothèque \\ Paul-Émile-Boulet de l'Université du Québec à Chicoutimi \\ Site web: http://bibliotheque.uqac.uquebec.ca/index.htm
}


Cette édition électronique a été réalisée par Jean-Marie Tremblay, bénévole, professeur de sociologie au Cégep de Chicoutimi à partir de :

E.E. Evans-Pritchard, La femme dans les sociétés primitives et autres essais d'anthropologie sociale. Traduit de l'Anglais par Anne et Claude Rivière. Paris : Les Presses universitaires de France, 1971, 248 pages. Collection : Bibliothèque de sociologie contemporaine.

Titre de l'ouvre originale : The position of women in primitive societies and other essays in social anthropology. (Faber and Faber Ltd, Londres, 1965).

Polices de caractères utilisée :

Pour le texte: Times, 12 points.

Pour les citations : Times 10 points.

Pour les notes de bas de page : Times, 10 points.

Édition électronique réalisée avec le traitement de textes Microsoft Word 2004 pour Macintosh.

Mise en page sur papier format LETTRE (US letter), 8.5' x 11 '')

Édition complétée à Chicoutimi, Ville de Saguenay, Province de Québec, le 6 janvier 2005. 


\section{Table des matières}

\section{Préface}

Note des traducteurs

Chapitre 1. - "La méthode comparative en anthropologie sociale" (1963)

Chapitre 2. - "La condition de la femme dans les sociétés primitives et dans la nôtre" (1955)

Chapitre 3. - "Structures politiques des peuples de langue nandi du Kenya" (1940)

Chapitre 4. - "Quelques expressions collectives de l'obscénité en Afrique” (1929)

I. Introduction

II. L'obscénité chez les Ba-Ila

III. L'obscénité chez les Ba-Thonga

IV. L'obscénité chez les Akamba

V. L'obscénité chez les Azande

VI. L'obscénité chez les Lango, les Didinga, les Lugbwara et les Ingassana

VII. Résumé des données et formulation du problème

VIII. Fonction sociale de l'obscénité collective

IX. Relation entre l'obscénité et les actes rituels

X. Fonction économique de l'obscénité

XI. Conclusion

Chapitre 5. - "L'état zande" (1963)

Chapitre 6. - "Le cannibalisme zande" (1960)

Chapitre 7. - La danse (1928)

La musique

Le chant

Les mouvements du corps

Schéma de la danse

Le leadership dans la danse

La fonction sociale de la danse

Le rôle de la danse dans les cérémonies religieuses

Chapitre 8. - "Signification sociale de la dot, en particulier chez les Azande" (1934)

Chapitre 9. - "La vie quotidienne des Nuer dans les campements de saison sèche" (1936)

Chapitre 10. - "Les noms de personnes chez les Nuer" (1948) 
Chapitre 11. - "Tribus et clans Luo" (1949)

$\frac{\text { I. }}{\text { II. }}$
$\frac{\text { III. }}{\text { IV. }}$
$\frac{\mathrm{V} .}{\text { VI. }}$
VII.

Chapitre 12. - "Le mariage coutumier chez les Luo du Kenya" (1950)

$\frac{\text { Simba }}{\text { Por }}$
$\frac{\text { Meko }}{\text { Diero }}$
$\underline{\text { Lupo }}$
Duoko
$\underline{\text { Powo }}$
$\underline{\text { Riso }}$
$\underline{\text { Sepo }}$
$\underline{\text { Miloha }}$
$\underline{\text { Ci Liend }}$
$\underline{\text { Lur }}$
$\underline{\text { Cieno }}$

Chapitre 13. - "Symbolique des noms de BOEUFS chez les Dinka Ngok" (1934)

Chapitre 14. - "Note sur la vengeance des esprits chez les Anuak du Soudan anglo-égyptien" (1953) 


\section{E.E. Evans-Pritchard (1963)}

\section{La femme dans les sociétés primitives et autres essais d'anthropologie sociale.}

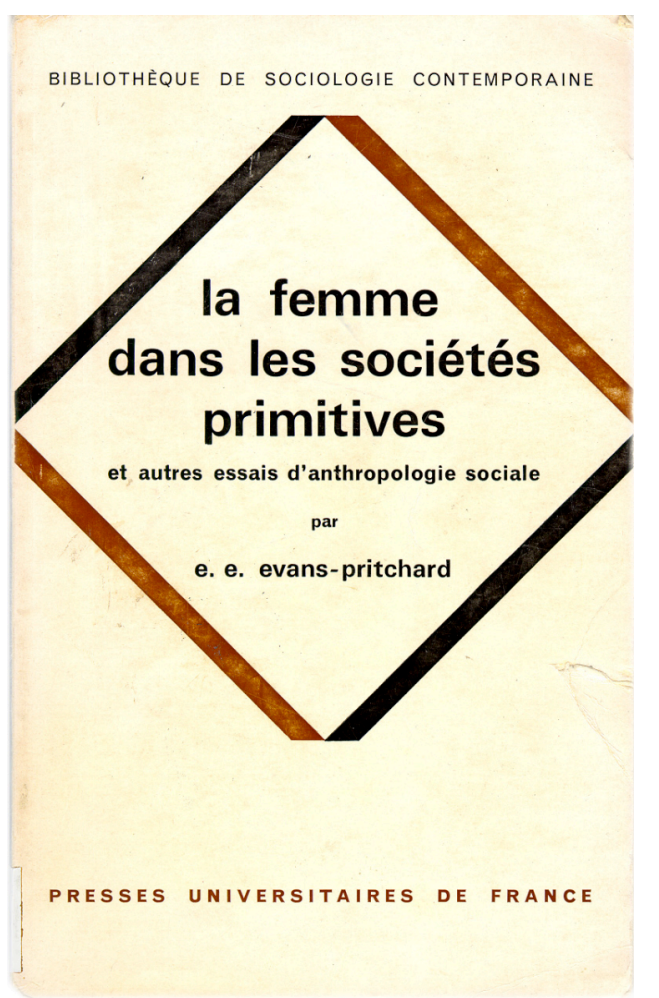

Traduit de l'Anglais par Anne et Claude Rivière. Paris : Les Presses universitaires de France, 1971, 248 pages.

Collection : Bibliothèque de sociologie contemporaine. 


\section{Préface}

Depuis une vingtaine d'années, le nombre des étudiants en anthropologie sociale s'est considérablement, accru, de même que l'intérêt suscité par cette discipline. Pour répondre à ce qui me semblait être un souhait général, je me risquai donc à publier quelques-unes de mes conférences et études sous le titre Essays in Social Anlhropology. J'étais plutôt pessimiste quant au succès de cet ouvrage, mais comme, en fait, il s'est révélé utile, j'ai décidé de rassembler une seconde série d'essais. Ce deuxième volume comprend, d'une part, deux conférences générales dans lesquelles je tente des analyses comparatives, et, d'autre part, divers articles, fruits de recherches sur le terrain, qui sont encore pour la plupart, m'a-ton dit, utilisés dans les universités. Ces articles décrivent des coutumes sans doute disparues, ou tout au moins en voie de disparition, chez les peuples est-africains. Le mélange intentionnel a pour but de montrer que l'anthropologue social ne doit pas se borner à étudier une seule société ou simplement quelques aspects de la vie sociale des peuples primitifs, mais qu'il doit effectuer de nombreuses recherches dans un grand nombre de sociétés. Les Azande, les Nuer, les Dinka et les Anuak sont des peuples du sud du Soudan. Les Nandi et les Luo vivent au Kenya. 
Des collègues et ceux dont j'ai eu l'honneur d'être le professeur m'ont souvent pressé de rééditer les articles que je présente ici. J'ai inclus les deux derniers, sur les noms de bœufs et la vengeance des esprits, pour les tirer de l'obscurité de quelques anciens numéros de revues car, à mon sens, les sujets dont ils traitent ne manquent pas d'intérêt.

Je n'ai apporté que quelques corrections mineures. Comme je l'ai noté dans mon premier volume, je ne m'exprimerais pas aujourd'hui de la même manière qu'il y a trente ou quarante ans, mais ce serait une erreur, je pense, de supprimer ce qui m'apparaît maintenant comme des imperfections et d'opérer un mélange de la pensée et du style d'alors avec ceux d'aujourd'hui. Il est évident que l'anthropologie sociale s'est modifiée et que j'ai moi-même changé. Aussi vaut-il mieux que les écrits de naguère restent ce qu'ils étaient dans l'atmosphère intellectuelle de l'époque. 


\section{Note des traducteurs}

Les noms propres de personne, de région, d'ethnie, de clan... ainsi que les mots vernaculaires, ont été orthographiés comme dans le texte anglais, plus proche que le français d'une écriture phonétique. Aussi le e de «Zande » se prononce-t-il é ; le u de «Babukur » ou de « Nuer» : ou; le gis de « Kipsigis » : guiz; le in de Dinka ne doit pas être nasalisé.

Nous avons observé la règle de l'invariabilité en genre et en nombre de ces noms propres, sauf, à l'instar de l'auteur, dans les chapitres sur l'État et le cannibalisme zande, où le pluriel de certains noms d'ethnies ou de tribus a été marqué, selon l'usage autochtone, par le préfixe A; exemples: un Mbomu, des Ambomu; un clan vongara, la dynastie des Avongara.

Employés comme adjectifs, ces noms propres ne portent pas la majuscule. Exemple : les Luo, le pays luo. Néanmoins, nous nous sommes permis de déroger à cette règle, en des cas exceptionnels, afin de lever une ambiguïté de sens ou de faciliter l'intelligence du texte.

A. et C. RIVIÈRE. 
La femme dans les sociétés primitives (1963)

\section{Chapitre 1 \\ "La méthode comparative en anthropologie sociale" *(1963)}

\section{$\underline{\text { Retour à la table des matières }}$}

Afin d'honorer la mémoire de L. T. Hobhouse, j'ai choisi comme sujet de cette conférence une méthode de recherche qu'il utilisa fréquemment. Vous estimerez peut-être qu'après avoir fait l'objet d'un exposé de Radcliffe-Brown ${ }^{1}$ en 1951, de Ginsberg ${ }^{2}$ et de bien d'autres depuis, une telle méthode ne méritait pas qu'on y revînt. En réalité, c'est seulement une fois à l'œuvre que je découvris l'abondance de la littérature parue sur ce sujet dans les dernières années. Sans espérer innover, je cherche seulement à mettre en relief certaines idées déjà exprimées par d'autres et à déterminer ma position. Je ne m'excuserai donc pas davantage d'une telle remise en question de la méthode comparative, car les implications en sont aussi fondamentales que cruciales.

Tout en circonscrivant mon champ à l'historique de la méthode comparative, notamment parmi les auteurs anglais, je limiterai mes commentaires à quelques exemples d'utilisation de

* Cette conférence fut prononcée le 14 mai 1963 à la London School of Economics and Political Science (L. T. Hobhouse Memorial Trust).

1 A. R. RADCLIFFE-BROWN, The Comparative Method in Social Anthropology, The Huxley Memorial Lecture, J.R.A.I., 1951.

2 Morris GINSBERG, Evolution and Progress, 1961, pp. 194-207. 
cette méthode (il ne sera fait mention ni de Frazer, ni de Max Müller, ni de Westermarck, comme vous l'espériez peut-être). Étudier la méthode comparative impliquerait une revue complète des ouvrages d'anthropologie. Et si on l'entend au sens le plus large, il n'est point d'autre méthode. La comparaison constitue sans aucun doute l'une des démarches essentielles de toutes les sciences et l'un des processus élémentaires de la pensée humaine. De toute évidence, si l'on doit émettre des jugements généraux sur les institutions sociales, on ne peut y parvenir qu'en comparant un même type d'institutions dans un grand nombre de sociétés. Bien entendu, semblable constatation ne date pas d'hier. Ce qui fut plus tard appelé méthode comparative était déjà utilisé par Aristote dans son analyse des différentes formes de constitutions, par Machiavel dans ses recherches sur les conditions qui déterminent le maintien au pouvoir ou la chute des princes (l'art de la politique) et par Montesquieu lorsqu'il étudiait le lien entre les institutions, valeurs, sentiments, climats et les diverses formes de gouvernements, appelées aujourd'hui types idéaux de gouvernements... pour ne citer que trois classiques. Ceux que nous pouvons appeler les précurseurs de l'anthropologie sociale et de la sociologie, Adam Ferguson et John Millar en Angleterre, Turgot, Condorcet et Comte en France, l'utilisèrent pendant tout le XVIIIe siècle. Mais leur méthode apparaît plus illustrative que comparative. Les exemples qu'ils citaient ne visaient qu'à corroborer des thèses issues d'une réflexion hâtive et non systématique. Notons aussi que la connaissance des sociétés primitives étudiées par les anthropologues sociaux était alors extrêmement rudimentaire, bien que ces sociétés aient fortement intéressé la philosophie des lumières.

McLennan, dans son Primitive Marriage (1865) et ses écrits ultérieurs, tenta le premier une étude systématique comparative des sociétés primitives, en puisant ses exemples dans les divers continents. Dans cet ouvrage, il veut démontrer que les groupes de petite dimension (stock groups), l'exogamie, le mariage par rapt, l'infanticide des filles, le système de parenté matrilinéaire et la polyandrie sont des institutions interdépendantes qui subissent, dans toutes les sociétés, des modifications, selon un ordre régulier, chaque société traversant les mêmes stades de développement social. Lorsqu'il n'observe pas la simultanéité de ces institutions, McLennan recourt à la preuve suspecte des «symboles », survivances ou « observances » archaïques, qui signalent qu'une coutume ou une institution existait autrefois. Ainsi, le mariage symbolique par rapt est une survivance prouvant l'existence antérieure du mariage par rapt proprement dit. Sa thèse comporte d'autres déficiences ; néanmoins, en bon juriste, il plaida sa cause avec logique. Quelques-unes de ces déficiences se situent dans l'élaboration même de la thèse : par exemple, il est difficile de concilier le mariage par rapt et ce qu'il estime en être la cause : l'infanticide des filles. Mais la plus sérieuse des critiques contre McLennan réside en ce que les faits n'étaient pas ce qu'il les supposait. Cependant, il ne pouvait probablement pas le savoir. Par exemple, Lowie ${ }^{1}$ mit en évidence le caractère non habituel d'un état d'hostilité permanente entre les peuples primitifs, la rareté de l'infanticide des filles et de la polyandrie. On sait aussi que le matriarcat n'existait pas dans la majorité des races primitives, que les Aborigènes d'Australie n'étaient pas tous matrilinéaires, que la parenté n'était pas nécessairement liée à la consanguinité, que le mariage et la famille n'étaient pas absents de la société primitive, etc. Toutes ces erreurs viciaient l'argumentation de McLennan.

La valeur et la faiblesse de la méthode comparative ainsi utilisée ressortent clairement du travail de pionnier de McLennan. Son mérite principal est d'avoir permis de séparer le

R. H. LOWIE, The History of Ethnological Theory, 1937, pp. 43-49. 
général du particulier, et, ce faisant, de nous fournir des concepts classificatoires importants pour le développement de l'anthropologie sociale. Ces concepts nous induisaient à chercher les causes générales de ce qu'il nous montrait comme étant des institutions du même type, recensées dans un grand nombre de sociétés dispersées à travers le monde entier : l'exogamie, le totémisme, le matriarcat, le mariage par rapt symbolique et l'association de celui-ci avec l'exogamie. D'autre part, la méthode comparative avait été principalement utilisée pour l'élaboration d'hypothèses historiques, qu'en l'absence de preuves historiques on ne pouvait considérer, selon toute probabilité, ni comme justes ni comme erronées. La méthode donna des résultats remarquables en philologie comparée, jurisprudence comparée et mythologie comparée. Mais ces résultats n'étaient dus, comme dans la biologie darwinienne, qu'à son emploi pour expliquer des termes de même origine. Autrement dit, cet emploi se limitait à l'étude d'institutions de peuples ayant une même origine sociale et culturelle. A défaut de preuves irréfutables, du moins atteignait-on à un degré plus élevé de probabilité. Ceci explique l'optimisme enthousiaste de l'historien Freeman ${ }^{1}$ pour la méthode comparative. Les hypothèses de McLennan se heurtèrent aussi aux faits ethnographiques. Comme l'indiquent ses carnets de notes, intéressants à comparer avec les derniers de Frazer, ce que l'on savait alors des peuples primitifs était trop insuffisant et trop incomplet pour justifier de vastes généralisations, et moins que toute autre celles qu'il tenta. Comme l'ont montré des éludes comparatives plus récentes, à l'échelle mondiale, il est impossible à un seul anthropologue, et même à une équipe d'anthropologues, de contrôler les sources ethnographiques portant sur des cultures et des sociétés aussi variées qu'hétérogènes. Et la difficulté ne fait que croître au fur et à mesure que les matériaux ethnographiques augmentent en quantité et en complexité.

Il convient de mentionner ici Herbert Spencer dont la contribution à notre sujet n'a eu d'égal que l'oubli dans lequel elle est tombée par la suite. Huit parties de sa Descriptive Sociology, commencée quelques années plus tôt, parurent entre 1873 et 1881 et n'eurent pas de suite, car leur publication l'avait presque ruiné. Il souhaitait démontrer comment les phénomènes sociaux sont liés entre eux, en classant les données de toutes les sociétés humaines dans des tableaux dont les titres permettaient une rapide comparaison. Les résultats de ce procédé, sur certains sujets, furent présentés dans les deux volumes de The Principles of Sociology (1882-1883). Spencer se donna beaucoup de mal pour prouver que toutes les sociétés humaines appartiennent à l'un on l'autre des deux groupes suivants : les sociétés militaires (qu'il détestait) caractérisées par la coopération forcée et des relations sociales fondées sur le rang, et les sociétés industrielles (qu'il approuvait) caractérisées par la coopération volontaire et par des rapports sociaux contractuels. Mais on pouvait à peine soutenir une typologie aussi simple. Même si une telle classification ne visait qu'à définir des types idéaux, une plus grande subtilité d'analyse s'imposait pour qu'elle acquière de la valeur. Ceci étant, j'ose dire que rien de valable ne résulta de sa tentative de démontrer les corrélations (congruities, un de ses mots favoris) entre la société de type militaire, la polygynie, l'assujettissement de la femme, la profusion du cérémonial (révérences, titres, insignes, etc.), et les corrélations entre la société de type industriel, la monogamie, le statut élevé de la femme et l'absence relative de cérémonial. Lorsque les faits le contredisaient, il disposait d'une formule de défense. En voici un exemple : Spencer croyait que les faits notés sur un échantillonnage de cinquante-deux personnes corroboraient son hypothèse selon laquelle les mutilations corporelles représentaient les signes de l'assujettissement, et qu'il existait donc une relation entre leur degré de gravité et le type de société considéré, que ces

E. A. FREEMAN, Comparative Politics, 1873, pp. I et 302. 
mutilations n'étaient pas caractéristiques des sociétés simples mais de ce qu'il appelle les sociétés composées, disposant d'une organisation militaire et d'un pouvoir politique (avec la subordination qui en découle). Lorsque les faits infirmaient ses dires, comme dans le cas des Boschiman et des Australiens, il se réfugiait derrière le prétexte d'une dégradation de la vie sociale parmi ces peuples. Même si l'on admire l'habileté et l'intégrité intellectuelle de Spencer, il n'en reste pas moins que ses conclusions relèvent de simplifications abusives, car il fonde ses classifications sur des approximations qualitatives grossières que dévalorisent souvent, comme dans tous ses écrits, des analogies biologiques inadéquates; les corrélations appuyées sur des preuves à la fois faibles et sélectionnées demeurent douteuses et vagues, et, qui plus est, si vastes et si générales que nul n'a jamais pu les utiliser. Ces preuves se trouvent cataloguées dans son énorme Descriptive Sociology, qui, pour autant que je sache, n'a jamais pu servir à des fins comparatives profitables, étant donné à la fois le curieux système de classification et la pauvreté de l'information ethnographique de l'époque. Le volume sur l'Afrique fut remis à jour par mon ami Emile Torday en 1930, mais apparemment sans grand profit, si bien que les mandataires de Spencer abandonnèrent la partie. L'ensemble reste une mine d'information délaissée.

Ainsi que nous le montre sa célèbre conférence de 1888 (vingt-trois ans après la parution du livre de McLennan), On a Method of Investigating the Development of Institutions; applied Io Laws of Marriage and Descent, Tylor sentit que les savants ne reconnaîtraient le statut scientifique de l'anthropologie qu'à condition qu'elle offre (ce dont McLennan ne s'était guère préoccupé, pas plus que Spencer d'ailleurs) une méthode d'analyse plus rigoureuse et des résultats chiffrés -improprement appelés résultats statistiques. Tylor voulait montrer que les recherches sur le développement des institutions pouvaient être inventoriées et classées. Il passa en revue les écrits portant sur les lois du mariage et de la progéniture d'environ trois cent cinquante peuples, et les classa en tableaux pour vérifier ce qu'on nomme généralement corrélations (les congruities de Spencer) et que lui appelle adhesions, c'est-à-dire que l'existence d'une certaine coutume entraîne celle d'autres coutumes dans un nombre de cas suffisant pour qu'il ne s'agisse pas de coïncidences dues au hasard. Ainsi pensait-il établir des explications à valeur universelle. En utilisant ce procédé, il s'aperçut que la coutume d'évitement était liée aux formes de résidence, et la coutume de technonymie aux deux à la fois (sans porter attention à la coutume de désigner les femmes par le nom de leur premierné). Il traita d'autres coutumes de la même manière : par exemple le lévirat (sans distinguer entre le lévirat et l'héritage des veuves par le frère du défunt), la couvade, le mariage par rapt, l'exogamie et le système classificatoire des nomenclatures de parenté. Son thème directeur visait à prouver que toutes les sociétés passent invariablement des institutions matriarcales aux institutions patriarcales.

De sérieuses objections s'élèvent contre l'usage que fit Tylor de la méthode comparative, qui présente aussi des problèmes difficiles à résoudre. En face d'une exception à la règle, par exemple lorsque la patrilocalité et le fait pour un mari d'éviter les parents de sa femme se rencontrent ensemble, comme dans bien des cas, il tente de contourner la difficulté à la manière de McLennan, qui, à mon avis, contredit toute sa conception des adhesions, en prétendant qu'il s'agit d'une survivance (notion «géologique » pour lui) d'un état de matrilocalité. Mais s'il considère le mode de résidence comme la constante et la conduite d'évitement comme la variable, on aimerait qu'il explique pourquoi la variable ne change pas quand la constante change, et pourquoi ce qui survit dans certaines sociétés ne survit pas dans d'autres. De plus, en lui supposant pour intention première de démontrer que toutes les 
sociétés passent du système matriarcal au système patriarcal, il convient d'observer d'abord que les peuples qui offrent les meilleurs exemples de système matriarcal, les Nayar, les Minangkabau et les Iroquois, sont loin d'être des peuples primitifs, et qu'ensuite, on peut douter de trouver un exemple certain de la séquence matriarcat-patriarcat au cours de l'histoire, exception faite du relâchement des liens de parenté dans les sociétés urbaines et industrielles modernes. Galton, à ce propos, estima essentiel de savoir dans quelle mesure les coutumes comparées étaient indépendantes, car elles pouvaient dériver d'une source commune et n'être que les duplicata d'un même original, comme ce pourrait être le cas avec la propagation de l'anglais et du système parlementaire. En outre, selon la remarque de Flower, la méthode devait reposer entièrement sur des unités de comparaison de même valeur. La monogamie chez les Veddah de Ceylan et la monogamie en Europe occidentale sont-elles des unités de même valeur? Le monothéisme islamique équivaut-il au monothéisme des Pygmées ? Une difficulté supplémentaire surgit avec la détermination du groupe dans la classification: les Aborigènes d'Australie représentent-ils un groupe ou plusieurs ? Dans ce cas, les Chinois ou les Anglais ne seront-ils qu'une unité correspondant à la horde Penan, par exemple ? Je n'ai jamais cru que, sur de tels sujets, les coïncidences « audelà du seuil de probabilité » aient eu la signification que leur attribuait Tylor. Il reste aussi à savoir si les corrélations qu'il croyait avoir établies n'étaient pas des corrélations entre des phénomènes concrets plutôt que discrets, s'il n'avait pas, en certains cas au moins, démantelé des ensembles puis démontré que les parties de ces ensembles qu'il avait isolées constituaient des touts. Si ses recherches avaient montré que l'aire de distribution des bananes est aussi celle de la couvade, ou que l'aire du lévirat correspond à celle des langues monosyllabiques, « au-delà du seuil de probabilité », elles auraient pu, en fait, poser un problème. De la façon dont il a procédé, on pouvait juger prévisibles les conclusions qu'il a atteintes par la statistique. Quelques autres déficiences appellent une brève allusion. On ne saurait tout exiger d'un pionnier de grand talent et d'esprit original, mais pour que les corrélations établies soient convaincantes et significatives, il faudrait dépasser les simples coïncidences et les simples différences, et dégager les variations concomitantes. Or Tylor n'a jamais publié les preuves sur lesquelles il avait étayé ses conclusions, si bien que nous ne connaissons ni les sources, ni les peuples lui ayant servi à fonder ses théories (la recherche des preuves de Spencer se révèle être aussi une entreprise difficile). Nous ne disposons par conséquent d'aucun moyen de vérifier l'exactitude des résultats revendiqués. Enfin, une autre difficulté que nous rencontrons tous, lorsque nous essayons de comparer les choses (les coutumes) plutôt que certains aspects ou certaines qualités de ces choses, provient de ce qu'elles peuvent, et à certains égards doivent, se ressembler, tandis qu'elles diffèrent sous certains autres angles, si bien que la classification, qui précède nécessairement la comparaison, dépend des critères choisis. Si les critères choisis diffèrent, les conclusions diffèrent.

Je ne fais que mentionner le nom du brillant et savant sociologue hollandais, S. R. Steinmetz, qui, à la fin du siècle dernier, espérait, grâce à l'analyse quantitative d'une énorme variété de données portant sur des sociétés et cultures du monde entier, parvenir à des conclusions significatives. On ne saurait affirmer que, même dans son célèbre ouvrage sur la vengeance et la haine dans les sociétés primitives: Ethnologische Studien zur ersten Entwicklung der Strafe (1894), il ait dégagé d'importantes corrélations à partir d'échantillons de travaux ethnologiques pourtant si soigneusement sélectionnés. La même critique vaut pour ses disciples, et notamment pour l'un d'entre eux, dont nous allons maintenant étudier la recherche, modèle du genre, publiée en anglais : Slavery as an Industrial System de Nieboer (ire éd., 1900). L'auteur y démontre que la forme d'esclavage dont il parle ne se rencontre ni 
parmi les chasseurs, ni parmi les pêcheurs - à l'exception des Indiens de la côte nord de Californie, où prévalent des conditions spéciales, particulièrement l'abondance de nourriture , ni chez les vrais pasteurs où l'esclavage, non seulement ne saurait constituer un avantage, mais pourrait être un désavantage. De même on ne l'observe pas là où la survie nécessite un capital, tels que les traîneaux et les chiens chez les Esquimaux, ni dans les pays où nulle terre n'est sans propriétaire, comme dans la plupart des îles océaniques : l'individu dépourvu de capital pour exploiter des ressources, ou dépourvu de ressources à exploiter, se doit de travailler pour les autres. Dans ces conditions, le travail est libre et la contrainte n'a pas lieu de s'exercer. Au contraire, lorsque le travail permet à chacun d'obtenir une quantité de nourriture suffisante pour lui-même et pour sa famille, c'est alors que la force s'avère nécessaire pour obliger un individu à travailler pour un autre. Telle est la thèse essentielle de Nieboer, et ses conclusions lui paraissent confirmées par une étude de l'histoire économique de l'Angleterre et de l'Allemagne. Mais toutes les difficultés précitées se retrouvent: l'insuffisance des sources (traitées avec un prudent esprit critique, il est vrai), le problème des définitions, par exemple des mots esclavage et servage, ainsi que celui des classifications : dans quelle mesure est-il significatif de classer les Indiens de Californie, de culture très développée, avec les Aborigènes d'Australie ? Il faut aussi noter la complexité des faits, ou, en d'autres termes, la multiplicité des facteurs en jeu, dont beaucoup ne sont pas d'ordre économique (comme le suppose Nieboer) et qui font douter de la corrélation entre l'esclavage et le régime économique. L'histoire de l'esclavage romain ${ }^{1}$, malgré l'imprécision des faits, ne semble pas appuyer sa thèse, pour ne citer qu'un exemple qui l'infirme. Il conviendrait donc d'apporter nombre de réserves. En tout état de cause, ses conclusions ne nous conduisent guère plus loin que nous pouvions nous y attendre, et encore faudrait-il confirmer qu'en règle générale le maintien de l'esclavage soit fonction de son utilité.

Un des mérites de Nieboer est d'avoir évité les présupposés évolutionnistes qui nuisaient à l'analyse des faits chez McLennan, Spencer et Tylor. Il déclare expressément que sa classification des types économiques ou plutôt bionomiques n'a pour but que l'étude des états et non des stades. Il en est de même de l'ouvrage de Hobhouse, Wheeler, et Ginsberg publié en 1915 (vingt-sept ans après la conférence de Tylor, et cinquante après le livre' de McLennan) : The Material Culture and Social Institutions of the Simpler Peoples. An Essay in Correlation. Ce livre représentait une nouvelle tentative d'établir des correspondances entre des états économiques, au sens large, principalement bionomiques, et certaines institutions sociales, dans une optique semblable à celle de Nieboer, mais avec une thématique bien plus large. L'enquête portait sur plus de six cents peuples, parmi les moins connus (Naturmenschen), s'étendait jusqu'au début des civilisations historiques, et menait aux conclusions suivantes: le développement économique s'accompagne, avec évidence, d'un progrès de l'organisation gouvernementale et de la justice publique ; le matrilignage et la matrilocalité dans l'ensemble prédominent légèrement parmi les chasseurs, le patrilignage et la patrilocalité dominent incontestablement chez les pasteurs, tandis que les principes patrilinéaires et matrilinéaires se contrebalancent, à égalité presque, chez les agriculteurs ; les chiffres démentent la corrélation, parfois avancée, entre système matriarcal et agriculture ; la coutume de la dot dans le mariage croît de façon notable avec le progrès de l'économie, et plus nettement encore chez les pasteurs que chez les agriculteurs; la pratique de la polygamie s'étend avec le développement des techniques et atteint son maximum chez les

W. L. WESTERMANN, The Slave Systems of Greek and Roman Antiquity, 1955; M. I. FINLEY (édit.), Slavery in Classical Antiqutly, 1960. 
pasteurs ; la condition de la femme lui est défavorable dans toutes les sociétés primitives, plus encore chez les chasseurs que chez les agriculteurs, mais, dans l'ensemble, elle est pire chez les pasteurs; le progrès des techniques et l'évolution sociale en général favorisent le développement de l'art guerrier, de même qu'ils accusent la subordination de l'esclave et du serf, et la domination des nobles le cannibalisme « réel » est un trait plutôt régional que culturel la pratique de l'infanticide semble diminuer avec le progrès culturel, plus particulièrement chez les pasteurs; le principe communautaire de la tenure des terres prédomine aux stades les plus bas de la culture et garde une légère prépondérance chez les pasteurs.

J'admire les auteurs de cet excellent ouvrage qui ne tentèrent pas d'exagérer leurs mérites, ni d'éliminer les exemples contraires à leur thèse en arguant de survivances, mais je ne pense pas que les résultats obtenus soient à la mesure du travail accompli. J'ai déjà fait allusion aux difficultés que présentaient de telles argumentations, les principales provenant, d'une part, du manque relatif de connaissances sur certains peuples étudiés, et, d'autre part, de données chaotiques, incomplètes et incertaines - même les récits les plus systématiques parus entre 1888 et 1915 seraient traités aujourd'hui avec la plus grande circonspection. De plus, l'étendue du champ de leurs recherches ne laissait pas aux auteurs la possibilité de contrôler suffisamment leurs sources. Je me permettrai de citer ici mon propre terrain de recherches : les Azande d'Afrique centrale dont Schweinfurth est considéré, à tort, comme le principal spécialiste. Ce peuple connu sous le nom de «Niam-Niam» se trouve, dans les classifications, divisé en trois (Azande, Niam-Niam, Adio) voire quatre groupes différents, si l'on y ajoute les Abandia. Surviennent alors les difficultés habituelles : isoler les unités de comparaison, préciser si les institutions appartiennent à la même classe, reconnaître les origines ethniques communes. Il faut aussi déterminer les influences des autres facteurs: facteurs sociaux, facteurs d'environnement, facteurs raciaux... afin de savoir quelle signification donner à une corrélation statistique entre des états de développement culturel et l'incidence numérique d'une institution ou d'une coutume. Hobhouse, Wheeler et Ginsberg avaient parfaitement conscience de ces difficultés et reconnaissaient à la première page de leur ouvrage que "pratiquement il n'était aucune généralisation sociologique qui n'achoppe sur un fait bizarre, pour peu qu'on prenne la peine de le chercher ${ }^{1}$. Une fois de plus, je soulignerai qu'en général les études de ce genre ne font que confirmer des conclusions prévisibles, aussi utiles et intéressantes qu'elles puissent être : à savoir, qu'au progrès économique correspond en gros une évolution du gouvernement, de la justice, de l'art guerrier, de l'esclavage, de la noblesse, de la propriété de la terre selon le rang de l'individu. A un autre égard, ces études n'aboutissent qu'à des corrélations qui, pour autant qu'elles aient un sens, ne révèlent pas les causes de phénomènes tels que, chez les pasteurs, l'infériorité de la condition féminine, la prédominance du principe patrilinéaire, la recrudescence de la polygamie et la quasi-absence de l'infanticide et du cannibalisme. La méthode de corrélation statistique ne peut que nous poser des questions sans nous en donner les réponses.

L'emploi statistique de la méthode comparative, sur des données provenant de tous les continents, a été abandonné chez nous et semble dépassé en Hollande. Cependant, en Angleterre, la recherche, à l'aide de l'analyse comparative, de lois et propositions universelles ne souffrant pas d'exception, continue d'être défendue par Radcliffe-Brown, un des

L. T. HOBHOUSE, G. C. WHEELER et M. GINSBERG, The Material Culture and Social Institutions of the Simpler Peoples. An Essay in Correlation, éd. 1919, p. 1. 
professeurs les plus célèbres et les plus influents en anthropologie; il n'utilise pas les statistiques et sa version de la méthode comparative n'est en pratique qu'un retour à la méthode d'illustration. En réalité, je crois que Steinmetz aurait relégué la plupart de ses écrits au rang de spéculations oisives et Nieboer les aurait considérés comme typiques de ce contre quoi il protestait : l'habitude qu'ont certains écrivains d'élaborer quelque explication plausible d'un phénomène social, et de chercher ensuite des illustrations pour la vérifier, tout en négligeant le reste des matériaux concernant le sujet. En voici quelques brefs exemples. Dans son article : «The Mother's Brother in South Africa », il avait peut-être raison de mettre en doute la notion de survivance, mais ses apports positifs ne me semblent pas, comme on l'a prétendu, un modèle de procédure scientifique; bien au contraire. Il essaie de montrer que, d'après ce qu'il appelle le principe d'équivalence des siblings (frères germains), le sentiment de tendresse envers la mère s'étend à son frère et le sentiment de respect envers le père s'étend à sa sœur ; mais il ne tente pas, dans cet article, de relier ce transfert au système de parenté tout entier (par exemple le frère de la mère est aussi le frère de la femme du père), aux droits de propriété, à l'autorité politique, etc. Les preuves sont sélectionnées, et de plus limitées à cinq illustrations (deux sources ne sont pas citées). Il ignore tous les autres peuples du monde, bien que chez tous il y ait des fils de la sœur et des frères de la mère. Jack Goody attira l'attention sur ces lacunes ${ }^{1}$.

L'argumentation de Radcliffe-Brown, qui s'élabore en cercle vicieux, a été invalidée par l'étude d'autres sociétés, notamment par les renseignements plus récents et plus détaillés de Malinowski sur les Trobriandais. Dans un autre essai, Radcliffe-Brown (dont l'optique sur les relations entre sociologie et histoire est celle de Comte et des théoriciens marxistes) déclare que la forme adoptée par la religion est liée à la forme de structure sociale, de sorte que, là où l'on rencontre un système lignager, on doit s'attendre à trouver un culte des ancêtres, comme en Chine et à Rome. Mais de nombreuses sociétés pratiquant le culte des ancêtres ne présentent pas trace de système lignager, et l'exemple le plus typique de système lignager : celui des Arabes Bédouins qui sont musulmans, contredit aussi sa thèse. Dans une autre étude, il affirme que lorsque l'homme, pour sa subsistance, dépend largement de la chasse et de la cueillette, les animaux et les plantes sont pris pour objets d'une « attitude rituelle »; un tel exemple ne sert qu'à illustrer une loi générale, à savoir : que tout objet ou événement qui influe puissamment sur le bien-être matériel ou spirituel d'une société, tend à devenir objet d'une « attitude rituelle ». Mais une telle assertion est erronée, à moins qu'on entende par «attitude rituelle» une quelconque considération, auquel cas l'expression perd toute sa signification. Bien que foisonnent en mon esprit les exemples qui contredisent cette loi, je n'en citerai qu'un : Needham m'a dit que les Penan n'ont envers le palmier sagoutier aucune attitude qui puisse être qualifiée de rituelle, alors que cet arbre est essentiel à leur subsistance. La loi générale, si ingénument énoncée, ignore, dans son application aux phénomènes totémiques, un grand nombre de données ethnographiques qui, en Afrique spécialement, la contredisent. Quant aux faits observes chez les pasteurs et les agriculteurs, ils ne l'appuient pas non plus. Un dernier exemple : Radcliffe-Brown affirme que là où les sociétés sont divisées en moitiés, les deux moitiés vivent en un état d'opposition qui s'équilibre, et il définit ainsi l'opposition : «Une combinaison d'accord et de désaccord, de solidarité et de différenciation. » Comment pourrait-il en être autrement ? C'est là un truisme contenu dans le mot moitié lui-même, et la même définition pourrait s'appliquer à n'importe quel groupe social. De semblables généralisations appuyées sur quelques exemples choisis,

Jack GOODY, The Mother's Brother and the Sister's Son in West Africa, J.R.A.I., 1959, pp. 61-88. 
ou bien sont trop générales au point d'en être dépourvues de signification, ou bien sont plus précises et reposent sur des preuves peu consistantes, sans tenir compte des contradictions. Je citerai ici le vieux dilemme du sociologue énoncé par Kroeber : «Lorsqu'il a réussi à trouver une formule tellement logique qu'elle ne souffre pas d'exception, à ce moment-là, elle s'éloigne tant des phénomènes que sa valeur opératoire s'annule ${ }^{1}{ }^{1}$.

En réalité, il est évident qu'hormis la validité restreinte de telles hypothèses, à mesure qu'elles visent à une application universelle, leur abstraction s'affine. On peut aller plus loin et dire que toute prétention à l'universalité requiert naturellement une explication historique ou psychologique, plutôt que sociologique, et par là même, fait échec à la sociologie dont le but est d'expliquer les différences plutôt que les ressemblances : par exemple, si l'on veut soutenir l'existence d'un nombre très réduit de thèmes et même l'unicité de thème dans les contes de fées, en les considérant en termes de structure fonctionnelle ${ }^{2}$, alors on doit presque abandonner une explication en référence à une typologie des institutions, à moins qu'on ne puisse montrer que des contes structurellement semblables prennent une signification différente chez des peuples différents, ce qui reste à prouver. Plus la prétention à l'universalité s'accroît, plus l'interprétation causale devient subtile et plus elle perd de son contenu sociologique.

J'aimerais mettre en relief l'importance des différences pour l'anthropologie sociale en tant que discipline comparative, car il faut noter que, dans le passé, on a souvent mis l'accent sur les ressemblances, comme dans The Golden Bough, alors que ce sont les différences qui semblent réclamer une explication sociologique. C'est là un problème compliqué, car les institutions doivent d'abord se ressembler sous certains aspects avant de se différencier sous d'autres, mais nous ne nous appesantirons pas pour l'instant sur ce problème. Une seule illustration éclairera le sens de ce que nous voulons dire. Si nous étudions les peuples méditerranéens, nous nous intéressons peut-être moins à leurs ressemblances, qui peuvent avoir des origines raciales, géographiques, psychologiques et historiques, qu'à ce qui les différencie, et c'est là que l'explication sociologique a toutes chances d'être pertinente. Nous nous demandons comment un Grec diffère d'un Espagnol, d'un Italien ou inversement, et pourquoi?

L'usage de la méthode comparative, que nous avons étudié jusqu'à maintenant, conserve encore la vogue en Amérique sous le nom de cross-cultural approach. Social Structure (1949) de Murdock en offre le meilleur exemple. Une critique approfondie de ce livre demanderait un long développement qu'il ne me semble pas mériter. Ses sèches classifications et ses définitions terminologiques m'apparaissent d'une valeur limitée, bien qu'on puisse l'utiliser comme guide pour la fréquence et la répartition de certaines coutumes. A propos de ses conclusions, on peut se demander avec Köbben (dont j'ai suivi les pas sur un terrain qu'il avait si bien exploré) si le jeu en vaut la chandelle. Aussi, suis-je d'accord avec Franz Steiner, Schapera, Needham et d'autres qui jugent ce livre plein de contradictions, d'affirmations et de suppositions sans fondement solide. L'étude statistique, qui porte sur deux cent cinquante sociétés, présente, en plus des déficiences déjà notées : échantillonnage pauvre, regroupement grossier, critères de classification arbitraires et inadéquats, un manque de discernement presque incroyable dans l'utilisation des sources. La plupart du temps,

\footnotetext{
A. L. KROEBER, History and Science in Anthropology, A.A., 1935, p. 561.

V. PROPP, Morphology of the Folktale, 1958 (paru d'abord en russe en 1928).
} 
Murdock ne possède qu'une seule référence pour chaque peuple, bonne, mauvaise, indifférente, et toujours en anglais. Il regroupe ces références comme si elles étaient toutes de même valeur, et utilise la même source pour plusieurs peuples sans se soucier de sa validité. Si les faits sont faux ou douteux, que dire des conclusions? Je n'en fournirai que deux exemples. On nous assure que l'introduction de l'Islam chez les Kababish du Soudan a détruit l'exogamie clanique en instaurant le mariage préférentiel avec la fille du frère du père » ( $p$. 48). Quelle preuve avons-nous de cette affirmation et que signifie-t-elle ?

Une fois de plus, je me reporte à mes recherches sur les Azande. Pourquoi choisir comme référence sur ce peuple le professeur et Mme Seligman, pour lesquels j'ai du respect et de la sympathie, mais qui n'ont vécu ni en pays zande, ni dans ses environs ? Pour savoir si les chiffres de ses tableaux tiennent compte des Azande, ou bien de tel autre peuple, beaucoup de travail s'avère nécessaire pour un résultat incertain. Toujours à propos des Azande, je rencontre cette affirmation: "Certains Azande de la haute noblesse peuvent épouser leurs filles » (p. 13) ; affirmation déroutante en ce sens que tous les membres du clan royal, des milliers d'hommes, peuvent cohabiter avec leurs filles s'ils le désirent, mais il est permis de se demander si cette cohabitation constitue un mariage. Puis (pp. 226 et 239) il fait mention des Azande comme d'un peuple où l'on ne signale pas de clans. Pourtant C. et B. Seligman (pp. 500-501) donnent toutes les informations nécessaires à ce sujet. Il est vraiment ironique de constater qu'ils écrivent : « Les clans azande sont légion. »

Je pourrais citer de nombreux exemples de l'usage erroné des sources, mais, en m'en tenant aux Azande, je dois mentionner un disciple de Murdock : Horton. Köbben, auquel je me réfère, remarque qu'Horton présente de nombreux exemples d'une exactitude douteuse (p. 143). Un spécimen particulièrement remarquable nous en est offert à propos des Azande. Il cite Seligman (p. 516) pour montrer que les rapports sexuels entre personnes non mariées (il veut dire avec des jeunes filles célibataires) n'entraînent pas de sanctions, puisque l'habitude en a été introduite par les Européens. Il ne donne aucune référence pour cette affirmation, prise en outre hors de son contexte, lequel montre clairement qu'au moment où les Seligman écrivaient, de tels rapports étaient adultères, puisque les jeunes filles, à leur nubilité, étaient presque toujours déjà mariées, au sens légal c'est-à-dire coutumier du terme. Cette utilisation des preuves me semble fort peu satisfaisante. Finalement, le paradigme de Murdock sur l'évolution sociale m'apparaît inintelligible en dépit des efforts considérables que j'ai faits pour le comprendre.

Quatre-vingt-quatre ans s'étaient écoulés entre la publication de l'ouvrage de McLennan et celui de Murdock, et il est bon de se demander ce qui a été accompli jusqu'à ce jour, après bientôt cent ans d'efforts visant à formuler des lois générales en anthropologie sociale, par la comparaison d'institutions collectées dans le monde entier, et plus spécialement par l'utilisation des statistiques. A mon sens, cet énorme travail n'a produit que fort peu de résultats sûrs et significatifs. Les efforts réalisés pour appliquer la méthode comparative avec une plus grande rigueur, en réduisant les recherches à des sujets limités ou à des sociétés d'un certain type, montrent bien que d'autres anthropologues partagent mon opinion. Il faut admettre que ces limitations de champ présentent leurs propres difficultés : par exemple, des sociétés d'un même type de structure peuvent se révéler très différentes du point de vue culturel, et inversement, des sociétés appartenant à la même aire culturelle peuvent offrir une plus grande diversité structurelle. Lorsqu'on rencontre des sociétés très voisines parleur structure, leur culture et leur environnement, il semblerait que l'on dispose des meilleures 
conditions pour une comparaison détaillée, contrôlée et intensive, qui contraste avec une étude statistique extensive ; mais, même alors, certaines difficultés précitées subsistent, telles que la valeur et la précision inégales des données.

Durkheim préféra procéder de cette manière en utilisant des données ethnographiques plus précises que ces informations rassemblées par accident, souvent inexactes, isolées de leur contexte, non contrôlables par l'étudiant et récupérées dans le monde entier. L'exemple de son professeur, Fustel de Coulanges, l'avait peut-être orienté dans cette voie. Sa propre étude sur la religion des Aborigènes d'Australie centrale offre un exemple typique de l'usage restreint de la méthode comparative dans les circonstances les plus favorables, tout comme les essais de Mauss sur les Esquimaux et ceux de Hertz sur la représentation de la mort. Nous pouvons citer chez nous les études de Maine sur les institutions juridiques indo-européennes et le travail de Robertson Smith sur la structure sociale et le sacrifice chez les Sémites.

Plus récemment, plusieurs d'entre nous, Schapera, Eggan, moi-même et d'autres professeurs, avons recommandé cette sorte de recherche comparative intensive et limitée, comme la mieux en mesure de fournir des résultats valables. Son champ d'étude, Schapera le délimiterait géographiquement, en songeant principalement à l'Afrique du Sud, où il fit des recherches si exceptionnelles. Sans aucun doute, cette délimitation géographique se révèle bien adaptée lorsque l'on rencontre des affinités culturelles, une parenté linguistique par exemple. Par contre, elle ne conviendrait pas si bien à la région que j'ai particulièrement étudiée, le sud du Soudan, en raison de sa grande diversité culturelle et sociale. Il serait nécessaire de sélectionner, à l'intérieur même de cette région, les Nilotes par exemple, bien que pour une comparaison intensive l'hétérogénéité de leur groupe en ce qui concerne l'environnement, la culture, l'histoire, aussi bien que le manque d'uniformité et l'insuffisance des données, m'aient paru trop importants pour que des résultats significatifs puissent en être dégagés. Même une tentative d'étude comparative sur les seuls peuples nilotiques du nord, les mieux connus, ne m'a pas permis d'établir des corrélations dignes d'être signalées. Je ne pense pas que, dans l'ensemble, cette sorte de comparaison, si adéquate soit-elle, ait donné ailleurs beaucoup de résultats en dehors d'une certaine possibilité de classification élémentaire par types. Celle-ci a plus de chance de mener à des conclusions historiques qu'à des lois, calquées sur celles des sciences de la nature, qu'ont souhaité établir tous les anthropologues utilisateurs de la méthode comparative. Ce but s'est affirmé de plus en plus nettement depuis Montesquieu (ou si l'on veut depuis Comte) jusqu'à nos jours. Il n'est que de citer les indications méthodologiques de Radcliffe-Brown basées sur des données ethnographiques insuffisantes, ou les assertions de Murdock, qui, jugeant avoir atteint le but, par un procédé statistique douteux, affirme que les éléments de l'organisation sociale «se plient à des lois naturelles propres, avec une exactitude à peine moins frappante que celle qui caractérise les permutations et les combinaisons d'atomes en chimie ou de gènes en biologie » (pp. 183, 259 et 283).

Les limites de l'emploi de la méthode comparative ont permis à l'étudiant, il est vrai, un plus grand contrôle de ses matériaux, mais il est aussi possible de dire que plus on réduit l'échantillonnage, plus une corrélation risque d'être ou une simple coïncidence, ou une cohérence logique, ou une compatibilité morale, que l'on pourrait prendre par erreur pour une interdépendance fonctionnelle. Pour citer un exemple: Gluckman fit de louables efforts afin de prouver que la rébellion et les autres formes de conflits servent à maintenir la suprématie du pouvoir royal, et que la forme de structure sociale détermine le degré de stabilité du 
mariage. Ces propositions contiennent l'argument implicite suivant: si les sociétés qu'il étudie vérifient sa thèse (ce dont je ne suis pas convaincu, même après les récentes modifications qu'il y a apportées), les conclusions doivent atteindre une valeur générale, sinon, comment fournir la preuve de la signification des corrélations supposées. Elles n'éclairent pas, il faut bien le dire, les luttes des Carolingiens pour le pouvoir ou les taux relatifs des divorces en Angleterre et en Espagne de nos jours. Aucune loi générale ne peut être formulée par ce procédé. La théorie de Durkheim et de Mauss, selon laquelle une seule expérience bien contrôlée suffit à établir une loi sociologique, n'a rien de convaincant en anthropologie sociale. Elle suffit pour une hypothèse, non pour une loi, si par «loi » on entend une généralisation ne souffrant pas d'exception. Un seul exemple négatif, qui ne peut être codifié dans les termes habituels, contraint à abandonner ou à reformuler la loi ; trouver un tel exemple ne réclame pas une longue recherche.

Cette réserve émise, on peut soutenir que les études comparatives à petite échelle ont été plus profitables que les très vastes études statistiques. On peut même aller plus loin et affirmer que l'étude intensive d'une seule société peut se révéler plus féconde qu'une comparaison littéraire à n'importe quelle échelle. Tous les anthropologues expérimentés savent que des observations sur le terrain permettent de tester beaucoup plus rigoureusement une théorie que des recherches littéraires. L'information nécessaire pour résoudre un problème fait habituellement défaut parce que ce problème ne s'était pas posé dans les écrits ethnographiques antérieurs. Prenons l'exemple de Malinowski. Lorsqu'il eut terminé ses recherches sur les Trobriandais, nul, moins que lui, ne se souciait de méthode comparative. Pourtant, serait-il exagéré de se demander si son compte rendu sur les institutions trobriandaises n'apportait pas davantage au développement de l'anthropologie sociale que toutes les ambitieuses études comparatives qui l'avaient précédé, ne serait-ce que parce qu'il détruisait nombre de propositions sociologiques admises depuis longtemps et encore fortement enracinées. En outre, il serait difficile de contester, si l'occasion s'en présentait, que les études particulières de cette espèce, effectuées depuis lors, ont davantage contribué au progrès de l'anthropologie que les études comparatives dont nous avons parlé. Et ce semble être particulièrement le cas des études témoins sur le terrain où la comparaison ne s'opère pas de manière implicite entre la propre société de l'anthropologue et celle qu'il étudie, mais entre deux sociétés primitives dont les modes de vie et de pensée diffèrent des siens propres.

Il est vrai que lorsque le capital théorique du passé sera épuisé, on court le danger de voir le sujet se réduire à une succession d'études ethnographiques isolées, auquel cas il n'y aurait plus de place pour l'anthropologie sociale en tant que discipline scientifique particulière. Radcliffe-Brown souleva un grave problème lorsqu'il écrivit : "Sans études systématiques comparatives, l'anthropologie deviendra de l'historiographie et de l'ethnographie » ${ }^{1}$. On ne voit que trop clairement ce qu'il veut dire, mais je crois qu'il ne tenait pas suffisamment compte du fait que les études anthropologiques prenaient une direction différente des études littéraires comparatives qui lui étaient familières et, qu'en tout cas, il poursuivit durant ses dernières années. La recherche littéraire n'a cessé de céder du terrain à ce que j'ai appelé quelque part, peut-être assez improprement, la méthode expérimentale: méthode comparative d'une certaine façon, puisqu'il n'en existe pas d'autres, mais d'une espèce toute différente, qui semble mieux convenir aux procédés habituels des sciences naturelles. Cette substitution de la recherche expérimentale sur le terrain à la recherche comparative littéraire 
constitue un des mouvements les plus importants de ce siècle pour l'anthropologie sociale. Par exemple, j'ai écrit un livre sur la sorcellerie chez les Azande. D'autres anthropologues peuvent étudier, et ont étudié, la sorcellerie dans des sociétés différentes. A la lumière de leurs écrits, on pourrait se rendre compte si certaines de mes conclusions ont des chances d'être généralisées, tandis que d'autres, si l'on considère les observations et les interprétations comme a priori correctes, ne seront valables que pour les Azande ou pour quelques sociétés particulières. Si l'on effectue un nombre suffisant de recherches sur ce sujet, on aboutira à des conclusions générales et certaines, auxquelles je ne voudrais pas cependant accorder le statut de lois universelles. Je ne vois pas quelle autre procédure adopter. Il va de soi que l'on n'obtiendra d'abondants résultats de cette manière qu'à la condition que chaque chercheur soit au courant des hypothèses avancées par les autres et soit préparé à les tester. Il ne doit pas les ignorer comme Kluckhohn qui, dans son étude sur la sorcellerie Navaho, ne tint pas compte de mon ouvrage antérieur. Puis-je me permettre de donner un autre exemple de mon propre travail ? Lorsque je commençai à étudier les Azande, je m'aperçus vite, qu'à bien des égards, les sortilèges et les mythes de leur magie différaient de ce que Malinowski avait décrit comme caractéristique de la magie trobriandaise. Je supposai que l'on pouvait expliquer ces différences par la divergence des règles qui présidaient à l'utilisation de la magie dans les deux sociétés. Ce n'était là qu'une hypothèse limitée dont j'ignore encore la pertinence Il serait facile à n'importe quel chercheur sur le terrain de la tester, bien que trente ans se soient écoulés depuis, mais autant que je sache, personne ne s'y est employé. La difficulté de progresser apparaît très nettement si chaque nouvelle recherche n'est pas basée sur des hypothèses, afin de les confirmer, de les infirmer ou de les modifier. Je suggérerais que c'est en essayant de résoudre des petits problèmes de cette espèce, et non en tentant de vastes généralisations, que nous progresserons, par apports fragmentaires et à pas lents il est vrai, mais en nous appuyant solidement sur les faits ethnographiques.

Ceci m'amène à conclure. Je suppose que nul ne contestera l'axiome suivant : il n'est pas d'autre méthode en anthropologie sociale que l'observation, la classification, la comparaison, sous une forme ou sous une autre. Rien de fondamental ne diffère dans la méthode utilisée par Montesquieu et dans celle étudiée par Radcliffe-Brown et d'autres. Mais depuis la parution de L'esprit des lois, deux cents ans se sont écoulés, pendant lesquels on peut se demander, une fois de plus, ce qui a été accompli par la méthode comparative et sous quelle forme. Peu de choses, certes, qui méritent d'être comparées aux lois dégagées dans les sciences naturelles pendant ces deux siècles. Nous ne nous sommes pas rapprochés de la mathématique sociale envisagée par Condorcet, ni des lois de succession et de coexistence que, selon Comte, la méthode historique (la méthode comparative) devait nous révéler. La méthode n'a pas tenu ses promesses.

Pour quelles raisons? La méthode est semblable pour toutes les sciences, seules les techniques varient : des phénomènes différents exigent des traitements différents. Pourquoi alors les sciences sociales demeurent-elles à la traîne ? On a dit parfois que la connaissance plus approfondie et plus sûre des faits permettrait d'établir des lois de la vie sociale, mais il semble que ce soit le contraire. Les anthropologues pouvaient spéculer sur les sociétés primitives, tant qu'elles étaient peu connues. Maintenant la difficulté s'accroît car les exemples négatifs, confirmés par la recherche de professionnels chevronnés, surgissent de toutes parts pour mettre en échec la théorie générale. L'historiographie peut ici nous secourir. 
Comme Collingwood le note ${ }^{1}$, les historiens positivistes avaient l'habitude d'affirmer que lorsqu'un nombre suffisant de faits serait amassé, les lois de l'histoire se dégageraient ; mais heureusement occupés à compiler des montagnes de faits nouveaux, ils en oublièrent finalement les lois.

Parmi d'autres raisons, on avance aussi la complexité des phénomènes pour rendre compte de la lente progression de l'anthropologie sociale. Comte, en dépit de sa verbosité, comprenait clairement la méthode scientifique. Il nous a enseigné que, dans la hiérarchie des sciences, une science ne pouvait se développer que lorsque la précédente s'appuyait sur de solides fondements. Donc, puisque la sociologie, la reine des sciences, se place au sommet de la hiérarchie, elle a été la dernière à naître, car elle a dû attendre le développement de la psychologie, qu'il a appelé physiologie cérébrale. La raison de cette séquence, avec, à une extrémité les mathématiques, à l'autre la sociologie, en est la complexité accrue des phénomènes, au fur et à mesure qu'on s'élève dans l'échelle, si bien que ceux qu'étudie la sociologie sont les plus complexes de tous. Il faut en outre reconnaître que, plus la complexité s'accroît, plus la généralité des concepts respectifs de chaque science diminue. Il est vrai aussi que les institutions les plus simples des peuples primitifs peuvent offrir une déroutante complexité, car les facteurs en jeu sont si nombreux et si variés qu'il serait difficile d'isoler les corrélations entre des variables indépendantes, en supposant qu'elles existent. Mais ceci n'explique pas suffisamment pourquoi l'anthropologie sociale, en dépit du nombre de recherches effectuées durant les quelques dernières décades, n'a pas atteint un niveau de généralisation plus élevé. On a dit qu'en anthropologie sociale il n'était pas possible de procéder à des expériences comme dans les sciences naturelles. Mais on peut objecter que certaines sciences naturelles ne disposent pas de possibilités expérimentales très étendues; que si nous n'utilisons guère la méthode de laboratoire, la grande variété des sociétés qui s'offrent à notre observation, de même que l'histoire des institutions, expérimentent pour nous, en dehors même de notre contrôle. De plus, l'élément expérimental joue un grand rôle dans notre recherche sur le terrain. On peut sans doute avancer d'autres raisons : par exemple que la méthode comparative a été utilisée à des fins trop ambitieuses, et que la comparaison a surtout porté sur les coutumes, sur les « choses », plutôt que sur des relations quantitatives entre des qualités ou propriétés. Cette accusation nécessiterait une autre conférence pour l'étayer.

Mais ces défauts et ces difficultés, isolés ou conjugués, ne justifient pas de façon convaincante l'insignifiance des résultats obtenus, face à l'ampleur du but fixé. Serait-il donc téméraire de mettre en question la notion de base, si longtemps admise, de l'existence de lois sociologiques telles qu'on les recherchait; de se demander si les faits sociaux, en dehors de leur complexité remarquable, ne diffèrent pas des faits étudiés par les sciences inorganiques et organiques, à tel point que ni la méthode comparative, ni aucune autre ne conduiront à la formulation de généralisations comparables aux lois de ces sciences. Il nous faut nous préoccuper de valeurs, de sentiments, de buts, de volonté, de raison, de choix, aussi bien que de circonstances historiques. Certains phénomènes sociaux, il est vrai, se produisent inconsciemment et même involontairement, le langage par exemple (ce qui peut expliquer que l'étude scientifique du langage aussi bien du point de vue historique que structurel soit plus précise que celle d'aucune autre activité sociale), mais on ne saurait affirmer la même chose de l'organisation d'une armée, de sa stratégie, de sa tactique. Dans une certaine mesure,

R. G. COLLINGWOOD, The Idea of History, 1946, p. 127. 
il n'est pas douteux, comme l'a remarqué Adam Ferguson, que bien que l'homme puisse choisir, il ne sait pas en définitive où le mène son choix. Tout le monde reconnaît que, dans le domaine de l'homme, on ne peut prédire l'avenir avec exactitude. Les découvertes et les décisions de demain qui influeront sur le développement futur nous sont inconnues aujourd'hui, au mieux pouvons-nous oser de vagues prévisions. Personne ne niera l'existence de principes limitatifs de l'organisation sociale, mais à l'intérieur de ces limites tout est possible. Les hommes choisissent continuellement la direction de leurs affaires, mais s'ils trouvent leur première décision désavantageuse, il n'est pas au-delà de leurs possibilités d'en prendre une deuxième qui la modifie. Le nier, c'est ignorer non seulement le rôle des valeurs et des sentiments, mais aussi celui de la raison dans la vie sociale. " La faute, cher Brutus, n'est pas dans nos étoiles. » En examinant la nature des institutions sociales, nous sommes passés du domaine de la loi naturelle à celui de la loi positive, pour reprendre la vieille distinction grecque entre la philosophie naturelle et la philosophie morale que Montesquieu a exploitée dans un célèbre passage : "L'homme, comme être physique, est, ainsi que les autres corps, gouverné par des lois invariables. Comme être intelligent, il viole sans cesse les lois que Dieu a établies, et change celle qu'il établit lui-même ${ }^{1}$.

Au cours de cette conférence, je n'ai pas eu l'intention de définir ce que devraient être les buts de l'anthropologie sociale, comment cette discipline pourrait ou devrait se développer, ni ce que sera son avenir, mais plutôt de décrire ce qui me semble avoir été ses buts et son évolution pendant les deux siècles écoulés.

Je ne crois pas que, quel que soit le résultat final, une existence passée à étudier les modes de vie et de pensée primitifs - il y aura quarante ans l'an prochain que je m'y consacre - soit mal employée, ni que la description de ces modes de vie puisse être inutile à la compréhension des êtres humains. Je n'ai pas de regrets. Peut-être devrais-je me considérer d'abord comme ethnographe, ensuite seulement comme anthropologue social, car je crois que la compréhension réelle des faits ethnographiques doit précéder toute véritable analyse scientifique.

Si je ne puis partager l'optimisme de mon professeur et ami Ginsberg (successeur de Hobhouse), lorsqu'il pense que, durant les cent prochaines années, le progrès des sciences sociales a des chances d'égaler celui des sciences biologiques et même celui des sciences physiques, mon scepticisme ne signifie pas que, à mon avis, nous devrions abandonner la recherche de ces constantes que les diverses formes de la méthode comparative peuvent permettre d'établir. Il serait intéressant de les trouver, mais si nous n'y réussissons pas, nous aurons au moins approfondi, au cours de notre recherche, notre compréhension de la société humaine.

Baron de MONTESQUIEU, L'esprit des lois, I, I. 


\section{BIBLIOGRAPHIE \\ du chapitre 1}

$\underline{\text { Retour à la table des matières }}$

COLLINGWOOD (R. G.), The Idea of History, 1946.

EGGAN (Fred), Social Anthropology and the Method of Controlled Analysis, A.A., 1954. , Social Organization of the Western Pueblos, 1950.

EVANS-PRITCHARD (E. E.), The Morphology and Function of Magic, A.A., 1929.

Witchcraft, Oracles and Magic among the Azande, 1937. FERGUSON (Adam), An Essay on the History of Civil Society, 1767.

FINLEY (M. 1.), édit., Slavery in Classical Antiquity, 1960.

FREEMAN (E. A.), Comparative Politics, 1873.

GINSBERG (Morris), Evolution and Progress, 1961.

GLUCKMAN (Max), Kinship and Marriage among the Lozi of Northern Rhodesia and the Zulu of Natal, African Systems of Kinship and Marriage, 1950.

$\longrightarrow$ Order and Rebellion in Tribal Africa, 1963.

GOODY (Jack), The Mother's Brother and the Sister's Son in West Africa, J.R.A.I., 1959, pp. 61-88.

HOBHOUSE (L. T.), WHEELER (G. C.) et GINSBERG (M.), The Material Culture and Social Institutions of the Simpler Peoples. An Essay in Correlation, 1915.

KLUCKHOHN (Clyde), Navaho Witchcraft, Papers of the Peabody Museum, 1944.

HÖBBEN (A. J.), New Ways of Presenting an Old Idea: the Statistical Method in Social Anthropology, the Curl Prize Essay, J.R.A.I., 1952.

KROEBER (A. L.), History and Science in Anthropology, A.A., 1935.

LEACH (E. R.), Review of Murdock's Social Structure, Man, 1950, n 169.

LÉVI-STRAUSS (Claude), Les limites de la notion de structure en ethnologie, Sens et usages du terme structure dans les sciences humaines et sociales, 1962.

LINT (Jan de) et COHEN (Ronald), One Factor Magic : a Discussion of Murdock's Theory of Evolution, Anthropologica, N.S. Z (1), 95-104, 1960.

LOWIE (R. H.), The History of Ethnological Theory, 1937.

MONTESQUIEU (baron de), L'esprit des lois, 1748.

MOORE (F. W.), édit., Readings in Cross-Cultural Methodology, 1961.

MURDOCK (G. P.), Correlations of Matrilineal and Patrilineal Institutions, Essays in the Science of Society, 1937. 
Social Structure, 1949.

Evolution in Social Organization, Evolution and Anthropology : a Centennial Appraisal, 1959, pp. 126-143.

NEEDHAM (Rodney), Notes on Comparative Method and Prescriptive Alliance, Bijdragen T.-L. \& V., 1962.

NIEBOER (H. J.), Slavery as an Industrial System, $2 e$ éd., 1910.

POPPER (Karl), The Poverty of Historicism, 1957.

RADCLIFFE-BROWN (A. R.), The Mother's Brother in South Africa, S.A.J.S., 1924.

, The Sociological Theory of Totemism, Fourth Pacific Science Congress, 1929.

, Religion and Society, The Henry Myres Lecture, J.R.A.I., 1945.

— The Comparative Method in Social Anthropology, The Huxley Lecture, J.R.A.I., 1951.

SCHAPERA (I.), Some Comments on Comparative Method in Social Anthropology, A.A., 1953.

SPENCER (Herbert), Descriptive Sociology, 1873-1881.

, The Principles of Sociology, 2 vol., 1882, 1883.

SPENCER (Robert F.), édit., Method and Perspective in Anthropology. Papers in Honor of Wilson D. Wallis, 1954.

STEINER (Franz), Review of Murdock's Social Structure, B. J. Soc., 1951.

STEINMETZ (S. R.), Ethnologische Studien zur ersten Entwicklung der Strafe, 2 vol., 1894.

TYLOR (E. B.), On a Method of Investigating the Development of Institutions ; applied to Laws of Marriage and Descent, J.A.I., 1889.

— Review of Steinmetz's, Ethnologische Studien, The Academy, janv. 1896.

WESTERMANN (W. L.), The Slave Systems of Greek and Roman Antiquity, 1955. 


\section{Chapitre 2}

\section{"La condition de la femme dans les sociétés primitives et dans la nôtre" * (1955)}

$\underline{\text { Retour à la table des matières }}$

Lorsque vous avez eu l'amabilité de me demander cette conférence, je vous ai répondu que ce serait pour moi un honneur, mais que j'ignorais tout de l'évolution des problèmes féminins en Angleterre, et j'ai suggéré que l'on me permette de parler d'un sujet qui me concerne professionnellement : la condition de la femme dans les sociétés primitives, et qu'en même temps, je tente de dégager les différences essentielles, qui m'ont frappé, ainsi que beaucoup d'autres anthropologues, entre la condition féminine, d'une part dans les sociétés primitives, et d'autre part dans la nôtre. Une réflexion sur la condition de la femme dans les sociétés primitives est d'autant plus intéressante qu'aucune étude sur l'évolution du statut féminin ne saurait l'omettre. En fait, une telle réflexion a souvent servi à appuyer l'un ou l'autre point de vue féminin, dans la littérature scientifique et polémique. Herbert Spencer prétendait qu'il n'est peut-être pas de meilleure façon d'éclairer le progrès moral de l'humanité

Ce discours à la mémoire de Mrs Fawcett fut prononcé le 25 octobre 1955 à Bedford College (Université de Londres) (a).

(a) A l'époque où je l'ai prononcée, je ne pensais pas que cette conférence méritât la publication. En la relisant, je trouve que certains aspects appellent quelques considérations supplémentaires. 
que d'opposer la condition de la femme chez les sauvages et sa condition dans les sociétés civilisées les plus évoluées.

Je ne crois pas, il est vrai, que la personne en l'honneur et en souvenir de qui cette conférence est donnée, ait été en aucune manière concernée ou intéressée par les peuples primitifs. Mais, en plus de ses talents intellectuels, Mrs Fawcett possédait une nature charitable ainsi que le sens de l'humour, et je pense que cela lui aurait plu et l'aurait amusée qu'une des conférences qui portât son nom eût pour thème une comparaison entre la condition de la femme dans notre société et celle de la femme dans les sociétés primitives. Je ne puis cependant affirmer avec la même assurance qu'elle aurait accepté certaines des conclusions auxquelles je suis parvenu, ou qu'elle aurait entièrement approuvé ma façon d'examiner et de commenter les faits. Elle était à la tête du mouvement féministe, et je dois avouer tout de suite qu'il se peut que j'encours votre censure, soit parce que je n'ai jamais vraiment compris le mouvement féministe, soit par mon manque d'enthousiasme à son égard. Néanmoins, les circonstances, et de ce fait les courants d'opinion, ont tellement changé qu'il se pourrait bien que Mrs Fawcett, si elle était encore en vie aujourd'hui, acceptât quelquesunes des idées que je vais émettre. Elle n'aurait certainement pas dédaigné d'en discuter, car c'était une personne des plus sincères et des plus honnêtes.

Quand, à la fin du siècle dernier et au début de celui-ci, les anthropologues Westermarck, Hobhouse, Ploss et Bartels, Mason, etc. -, qu'ils appartiennent à un groupe ou à un autre, par la comparaison de récits sur les sociétés primitives du monde entier, tentèrent de déterminer la condition de la femme dans ces sociétés, tout ce que l'on en connaissait se limitait à des relations de voyageurs, de missionnaires, d'administrateurs. Ces récits, toujours superficiels par rapport aux études modernes, abondaient fréquemment en préjugés. En réalité, ils nous permettent souvent de comprendre la société victorienne au moins autant que les sociétés primitives qu'ils décrivent, et il est intéressant de comparer les impressions produites par les peuples primitifs sur les esprits victoriens avec celles produites sur les esprits du XVIIIe siècle. Quand les voyageurs parlaient du statut de la femme dans les sociétés primitives, ils se référaient évidemment au statut de la femme en fonction de celui de l'homme. Si l'observateur portait un jugement - ce qui était toujours le cas -ce jugement dépendait de la conception qu'il se faisait des rapports réels ou idéalement souhaités entre les sexes, dans sa propre société et sa propre classe, au moment où il écrivait. Se référant a ces modèles, il voyait et décrivait la condition féminine comme supérieure ou inférieure, généralement inférieure par rapport à celle de la société victorienne.

Il convient de conserver à l'esprit que les relations sur la vie sociale des peuples sauvages, sur lesquelles les premiers anthropologues basèrent leurs conclusions, étaient généralement écrites par des hommes, et des hommes de la bourgeoisie, ayant souvent, lorsqu'ils étaient originaires d'Angleterre, une formation évangélique. Pour juger du statut de la femme sauvage, leur point de comparaison n'était pas la femme et la fille de l'ouvrier agricole ou industriel, mais leur propre mère et leur propre sœur. Leurs observations étaient alors groupées par des anthropologues issus, dans l'ensemble, du même milieu, et qui, de plus, se considéraient comme des progressistes, à l'avant-garde de la bataille livrée contre les dernières citadelles de la superstition et du privilège. Ils tendaient, en conséquence, à parler des coutumes et croyances sauvages, le regard fixé sur les changements qu'ils désiraient apporter dans leurs propres institutions. Les preuves étaient la plupart du temps si incomplètes et si contradictoires que l'auteur, par la sélection qu'il opérait, la présentation 
qu'il en faisait et l'importance qu'il leur donnait, pouvait largement discréditer ce à quoi il était opposé. On peut citer les exemples de Engels, quant à la famille primitive et à l'idée de propriété, et ceux de Frazer quant aux religions primitives.

Il faut aussi se souvenir que les anthropologues de cette période subissaient fortement l'influence des écrits de Darwin et d'Huxley. Rien d'aussi facile que de construire un plan d'évolution sociale ou super-organique à partir des théories de l'évolution biologique, et Herbert Spencer, notamment, ne s'en priva pas. En toute logique, si on avait posé le postulat de l'évolution biologique de l'homme, il devait y avoir aussi évolution sociale et culturelle; et le statut de la femme, d'une manière ou d'une autre, devait s'intégrer dans le plan, ce qui ne présentait pas de grandes difficultés pour les anthropologues victoriens, dont une des préoccupations favorites était les relations entre sexes. En fait, seule la religion pouvait rivaliser d'intérêt pour ces puritains incroyants. De toute évidence, puisque l'homme descendait de quelque ancêtre simiesque, sa vie sexuelle avait dû, à un certain moment, être elle-même simiesque, et la tâche des anthropologues était de montrer quelle place tenait la caverne du singe par rapport au salon victorien. On pouvait spéculer sur les premiers stades de ce développement, mais la ligne générale en était claire, puisque les points extrêmes étaient déterminés : la guenon et la «lady» victorienne. Il y eut les hordes d'Akinson, l'hétairisme de Bachofen, les groupes de petite dimension (stock groups) infanticides de McLennan, la promiscuité primitive et les groupes syndiasmiques de Morgan et de Sir John Lubbock, etc. Pour saisir quels niveaux de fantaisie pouvaient atteindre ces spéculations, il suffit de lire: The Position of Women in Primitive Society (1914) de C. Gasquoine Hartley, un de mes amis de jeunesse, qui était la personnification même des premiers progressistes édouardiens.

Les recherches des anthropologues ont désormais mis en évidence l'inexistence des formes sociales naguère postulées. Il est également clair qu'aucune recherche des préhistoriens, de quelque importance qu'elle soit, ne nous dira jamais ce qu'ont été les conditions sociales des premiers hommes. Par ailleurs, un changement complet s'est produit dans l'orientation des anthropologues. Le temps n'est plus, du moins pour le moment, où l'on fabriquait des théories évolutionnistes purement spéculatives et incontrôlées. Il est aussi passé le temps d'un féminisme et d'un antiféminisme exacerbés, éléments d'une bataille dans laquelle les protagonistes des mouvements appelés progressistes et leurs opposants se confondaient en une mêlée générale. Est-il une jeune personne qui puisse aujourd'hui lire Three Guineas de Virginia Woolf sans se demander quelle pouvait bien être la cause de tant d'indignation? Comme le note Mrs Fawcett dans son autobiographie, ce sont certainement des « problèmes désuets et anachroniques ».

La voie est maintenant libre pour examiner comment la condition de la femme varie, si elle varie, avec les types de culture et de structure sociale, sans qu'il soit besoin de prendre les armes au nom de qui que ce soit ou de quoi que ce soit. Les sociétés primitives nous fournissent un champ d'observation fécond de par leur nombre et leur variété. Leur importance découle autant de cette raison que du fait qu'elles soient primitives, pour qui s'intéresse à la condition sociale de la femme. Malheureusement aucun travail comparatif systématique n'a été entrepris récemment sur ce sujet. Si l'on désire des renseignements il est nécessaire d'accomplir la tâche difficile du recours aux sources, à savoir : les nombreuses monographies ethnographiques. Il se peut que le manque d'intérêt explique, cri partie au moins, l'absence de tentative en vue de regrouper les résultats de la recherche moderne en 
une seule étude, le statut de la femme dans notre société ayant cessé d'être un problème capital. Quoi qu'il en soit, je souhaiterais vous soumettre quelques réflexions sur ce sujet.

Il est difficile, sinon impossible, de juger objectivement de la condition de la femme dans une société primitive quelconque ou dans les sociétés primitives en général. En définitive, tous nos jugements se fondent sur nos propres opinions et habitudes. Sans une étude approfondie, ils risquent en outre d'être superficiels, et basés sur des apparences qui nous sont étrangères, plutôt que sur la réalité sociale qui les sous-tend, ou, en d'autres termes, sur l'expression culturelle des relations entre les sexes, plutôt que sur le contenu psychologique et moral de ces relations. Nous observons, par exemple, que dans certaines sociétés les femmes rampent devant leur mari ou que les personnes d'un sexe ne mangent pas en présence de l'autre. Si nous considérions ces comportements, selon notre propension, comme les signes de l'abjecte servilité féminine ou d'une attitude d'extrême réserve, voire d'hostilité entre les sexes, nous tirerions des conclusions entièrement erronées.

A partir de telles évidences, les théoriciens de l'époque victorienne, Herbert Spencer notamment, admettaient généralement que, dans les sociétés primitives, la femme était un bien que l'on achetait et vendait, qu'elle était traitée comme une esclave ou même comme un animal, qu'elle ne jouissait ni de la sympathie ni du respect. On pensait que les relations entre les sexes ne dépassaient guère celles des bêtes, et que le mariage, comme nous l'entendons, n'existait pas. Trop souvent, ces jugements, à caractère fortement subjectif, ne reposaient que sur des récits très fragmentaires et se calquaient sur les modèles quelque peu arbitraires de nos propres sentiments.

Il est vrai que l'on peut avancer certains critères apparemment objectifs du statut de la femme, à savoir par exemple, que, lorsqu'il y a homicide, la compensation versée pour la femme est inférieure à celle versée pour l'homme, que les deux sexes n'ont pas de semblables facilités pour divorcer ; mais ces critères peuvent n'être pas considérés comme réellement objectifs, car ils fixent ce que le statut de la femme devrait être. Néanmoins, si l'on veut aboutir, ne serait-ce qu'à des tentatives de conclusion, afin de savoir si le statut de la femme dans les sociétés primitives lui est favorable ou non (d'après nos modèles), on doit se servir de tels critères. Un des essais les plus complets effectué dans ce sens a été celui d'Hobhouse, Wheeler et Ginsberg: The Material Culture and Social Institutions of the Simpler Peoples (1915). Ces auteurs utilisent une grande variété de critères, comme Nieboer avant eux, mais ils attachent plus de poids aux droits garantis à la femme par la coutume et par la loi : ainsi se préoccupent-ils de savoir si le mari a le droit de battre sa femme, si la femme est protégée par la loi ou par sa famille, si la femme exécute les gros travaux à la maison, si la femme peut jouer un rôle de chef ou prendre part aux conseils tribaux. Les conclusions auxquelles ils ont abouti, semblables dans l'ensemble à celles de Nieboer, montrent que la condition de la femme, dans les sociétés les plus simples, lui est défavorable, et que, de ce point de vue, il n'existe pas de grandes différences entre chasseurs, agriculteurs et pasteurs. La méthode d'estimation, basée sur des données insuffisantes, comme les auteurs l'ont reconnu, ne permettait pas de détermination quantitative, ni de distinction entre ce que nous appelons les droits du mari et de la femme, et leurs relations réelles. Ils ne pouvaient non plus prendre en considération les nombreux impondérables qui, plus que la loi et même la coutume, déterminaient les relations entre époux. 
Les impondérables furent justement mis en valeur par un célèbre anthropologue américain, Robert H. Lowie, dans son excellent ouvrage : Primitive Society (1921). Il admet que dans la grande majorité des sociétés, non seulement dans les plus simples, mais aussi dans les plus complexes, la femme, d'après nos standards moraux les plus avancés, a une position moins désirable que l'homme. Il estime aussi que l'opinion populaire, ainsi que Spencer le supposait, selon laquelle le statut de la femme est un indice de développement culturel, se trouve en complète contradiction avec les données ethnographiques. Cependant, le passage de son livre, sur lequel je veux attirer votre attention, concerne les impondérables. Il écrit: "Les relations entre homme et femme sont multiples et il est dangereux d'en surestimer un aspect particulier. A la théorie, on ne doit attacher qu'une moindre importance. Certes, elle influence la pratique, mais seulement dans une certaine mesure. Théoriquement, le musulman Kirgiz peut divorcer d'avec sa femme à son gré. Pratiquement, il ne le fait que très rarement. La métaphysique chinoise identifie au mal le principe féminin de l'univers, et le statut légal de la femme est d'une abjecte infériorité. Cela n'a pas empêché bon nombre de Chinoises d'établir leur suprématie sur leur foyer par la simple force de leur personnalité, ni de jouer un rôle appréciable dans la littérature et les affaires. Autrement dit, il est important de préciser ce qu'énoncent le droit coutumier ou écrit et la théorie philosophique sur les droits et les devoirs de la femme. Mais il est encore plus important de savoir si la pratique sociale se conforme à la théorie ou la dépasse, comme il arrive fréquemment. »

Dans l'ensemble, les découvertes des anthropologues modernes s'accordent pour démontrer que le statut de la femme parmi les peuples primitifs a été mal compris et sousestimé. Par exemple, on admet maintenant que l'assimilation de la dot à une vente est une grossière erreur d'interprétation. Les études les plus autorisées sur les peuples primitifs, durant ces dernières années, tendent à mettre l'accent sur l'influence de la femme, sur son habileté à se défendre, sur l'estime dans laquelle on la tient, et sur l'importance de son rôle dans la vie sociale. Ainsi, pour citer à nouveau R. H. Lowie, celui-ci soutient, à propos des Indiens Crow d'Amérique du Nord, que : « la femme n'est pas du tout un bien meuble », que «sa condition est loin d'être défavorable», $\mathrm{qu}^{\prime}$ «il est peu d'interdits religieux la concernant », que «socialement elle jouit d'une assez grande liberté » et que «dans l'ensemble, la femme Crow bénéficie à la fois d'une situation stable dans la vie tribale et d'une bonne part de ses avantages ». Bien qu'on ne puisse affirmer la même chose de tous les peuples primitifs, on pourrait trouver, à propos de bon nombre d'entre eux, des assertions similaires dans la littérature moderne.

De peur que ces opinions ne soient considérées que comme le reflet d'une complaisance masculine, je citerai des conclusions semblables d'anthropologues féminins. Par exemple, Phyllis Kaberry, de l'Université de Londres, montre de manière indubitable, et contrairement à ce qu'on a souvent dit et cru, que les femmes des hordes aborigènes d'Australie s'intéressent à toutes les activités sociales et y participent souvent efficacement, que l'homme et la femme sont, au plein sens du terme, des partenaires avec des devoirs et des privilèges réciproques, au sein d'une union la plupart du temps stable. Dans une étude plus récente sur la condition économique de la femme chez les Bamenda du Cameroun britannique, le même auteur montre en quelle haute estime on tient la femme, de quelle liberté et de quelle responsabilité elle jouit au moins dans certaines sociétés ouest-africaines. Pour confirmation, je vous renvoie aux écrits de Ruth Benedict, de Margaret Mead et d'autres anthropologues féminins. 
Cependant, la question la plus pertinente n'est peut-être pas celle de la supériorité ou de l'infériorité de la femme dans les sociétés primitives par rapport à notre propre société. Si nous nous demandions plutôt sous quels angles diffère la condition féminine, il est plus vraisemblable que nous apprendrions quelque chose d'intéressant tant sur les sociétés primitives que sur la nôtre. Avant d'essayer de répondre à cette question, il me faut émettre quelques observations générales. Lorsque j'évoque la condition de la femme dans notre société, j'en parle telle qu'elle apparaît aujourd'hui en Angleterre. De toute évidence, la situation en Angleterre s'est modifiée, sous bien des aspects, au cours des diverses périodes de l'histoire ; et à certains égards, elle diffère énormément aujourd'hui de celle de beaucoup de pays d'Europe et d'Amérique. Lorsque je parle du statut actuel de la femme en Angleterre, il me faut m'appuyer en grande partie sur mon expérience des gens avec lesquels j'ai vécu ou que j'ai rencontrés, car je n'ai pas été capable de découvrir un livre que l'on puisse considérer comme une étude sociologique du statut de la femme dans la société anglaise contemporaine. En fait, un article récent de McGregor (Université de Londres) dans The British Journal of Sociology (1955), traitant des études consacrées à la condition de la femme en Angleterre entre 1850 et 1914, m'a convaincu de l'absence de toute recherche de ce genre. Naturellement, les ouvrages sur la femme abondent, je suppose même que la plupart des livres parlent des femmes, mais je songe plutôt à ces œuvres qui traitent spécifiquement de la femme, ces œuvres nombreuses pour ou contre ce qu'on a coutume d'appeler le mouvement féministe, ces œuvres sur la femme en général ou sur ce qu'on juge être la féminité. En réalité, au cours de mes lectures en vue de cette conférence, j'ai passé de bien agréables heures, dont je vous suis reconnaissant, en compagnie de remarquables dames anglaises du siècle dernier, qui m'ont beaucoup appris : Maria Jewsbury, Harriet Martineau, Elisabeth Fry, Angela Burdett-Couts, etc. ; j'ai lu des historiens, des satiristes, des romancières. Mais pour valables que soient les indications que l'on rencontre dans les histoires, les biographies, les belles-lettres et les romans, elles ne fournissent pas les preuves permettant de dégager des conclusions sociologiques adéquates sur la femme moyenne dans la société anglaise. Comme je n'ai mené aucune étude sociologique personnelle dans ce pays, je suis dans une situation certes pas meilleure, peut-être même pire que celle de mon auditoire, pour émettre des affirmations. Si l'on souhaite dépasser ses propres impressions et ses lectures générales, il faut, ici encore, recourir à des sources variées, ce que je n'ai pu faire moi-même que de façon superficielle.

Lorsque je parle de sociétés primitives, et je crois être mieux placé que vous pour juger, puisque j'ai passé des années à les étudier sur place et dans la littérature, je dois signaler qu'étant donné la multitude de sociétés de cette espèce, il est difficile de généraliser. Cependant, bien qu'on trouve toujours des exceptions, ce que je dis des sociétés primitives est vrai en général, c'est-à-dire est vrai de la plupart des sociétés primitives pour lesquelles nous disposons d'informations suffisantes. Il convient d'ajouter aussi que la majorité des assertions concernant la vie de la femme primitive s'expliquent plus par son appartenance à des sociétés de technique et d'économie rudimentaires que par sa qualité de femme. Aussi est-il difficile de comparer la condition de la femme dans notre culture et dans notre société avec sa condition dans des cultures et des sociétés primitives, sans comparer les cultures et les sociétés elles-mêmes sous maint autre aspect. Naturellement, la femme aborigène australienne a une vie plus pénible et socialement plus restreinte que nos sœurs et nos filles, mais il n'en va pas différemment de l'homme aborigène comparé à nos frères et à nos fils. 
Il me semble qu'un des faits qui frappe d'abord l'étranger chez un peuple primitif, c'est l'absence de femme adulte célibataire. Toutes les filles trouvent un époux et se marient, à notre avis, habituellement très jeunes. Si l'on remarque, par ailleurs, que dans une société de technique et d'économie rudimentaires, les travaux domestiques occupent la femme à plein temps, la charge des jeunes enfants n'entrant même pas en ligne de compte, on s'aperçoit, sans qu'il soit besoin d'autre considération, de la différence essentielle entre la femme moderne civilisée et sa sœur sauvage. Chez nous, la femme peut choisir entre le mariage et le célibat. Si elle opte pour le célibat, elle peut consacrer sa vie à l'enseignement, à la recherche, à l'administration, aux bonnes oeuvres, etc., ou bien si elle se marie, elle peut allier sa vie conjugale avec une profession, un emploi ou avec toutes sortes d'activités intéressantes hors de son foyer. La femme primitive n'a pas le choix, et étant donné les devoirs qui accompagnent le mariage, elle est rarement en mesure de participer à la vie publique. Mais si, de notre point de vue, nous pouvons la considérer comme désavantagée à cet égard, elle ne se considère pas comme telle, et n'envie pas ce que nous estimons être les privilèges masculins. Elle ne désire pas que les choses soient autrement, et elle serait fort étonnée de savoir que, dans notre société, beaucoup de femmes sont célibataires et sans enfant.

Dans les sociétés primitives, la possibilité ne s'offre à toutes les femmes d'acquérir un mari qu'en raison de l'autorisation quasi générale et de la pratique, à divers degrés, de la polygamie. En conséquence, toutes les femmes peuvent se marier, et la coutume permet aussi aux veuves de se remarier sans difficulté, en règle générale souffrant exceptions, avec un proche parent du mari décédé. Ceci constitue une autre différence évidente entre ces sociétés et la nôtre, car lorsque je dis que, dans notre société, une femme peut choisir de se marier ou non, j'entends par là qu'elle pourrait, si elle le voulait, à l'instar de beaucoup de femmes, refuser le mariage. Mais statistiquement, dans une société monogame, cette affirmation est fausse ; ce fut d'ailleurs l'un des principaux arguments féministes et l'un des plus efficaces : puisque beaucoup de femmes de notre pays ne pouvaient se marier, de plus grandes possibilités de s'engager dans des professions et des activités publiques devaient leur être accordées. L'argument ne s'appliquait en fait qu'à la bourgeoisie. Comme Mrs Craik l'écrivait: «Le mal principal qui ronge la vie de la femme est le besoin d'activité », d'une activité digne et respectable.

J'ai entrepris une comparaison entre la femme dans les sociétés primitives et dans la nôtre à propos du mariage, et notre débat devra d'abord porter sur quelques différences entre les relations du mari et de la femme dans les deux types de sociétés. La femme primitive adulte est avant tout une épouse dont la vie se concentre sur sa maison et sa famille. Nous ne pouvons comparer le statut de la femme que dans deux types de sociétés où la femme a un rôle comparable. Il n'est pas possible, par exemple, de comparer la condition de la femme britannique dans l'industrie avec celle de la femme primitive, parce que les peuples primitifs ne possèdent rien de comparable à l'industrie moderne. Mais les institutions matrimoniales et familiales forment les institutions de base des sociétés primitives comme de la nôtre. Dans ce domaine, on peut donc opérer quelques comparaisons, car nul n'a encore décelé un peuple primitif dépourvu d'une forme quelconque de mariage et de famille.

Un étranger en visite dans une société primitive observera que les parents ont autant d'enfants qu'il leur est possible. Rares sont les pratiques d'infanticide et d'avortement. Aucune femme ne demeure volontairement sans enfant et la stérilité totale est en vérité le pire des malheurs qui puisse lui arriver. La grande majorité des femmes donnent le jour à plusieurs 
enfants, mais beaucoup meurent en bas âge, La femme primitive ignore les moyens contraceptifs et je suis persuadé que si on lui en parlait, elle s'étonnerait fort d'apprendre que certaines personnes limitent le nombre de leurs enfants. Elle en désire autant que possible, en partie parce que la mère et le père d'une famille nombreuse jouissent d'un grand prestige, mais aussi parce que fils et filles aident de différentes manières leurs parents dans l'entretien de la maison, renforcent la famille par les alliances du mariage et sont une assurance pour la vieillesse. La situation se présente très différemment en Angleterre de nos jours, où les enfants constituent une dépense importante pour leurs parents, qui n'en peuvent attendre qu'une faible, sinon aucune, contrepartie. Telle est certainement une des raisons, parmi d'autres, pour lesquelles la contraception est aujourd'hui pratiquée par une grande partie de la population et à tous les niveaux sociaux. Si notre société devait se composer surtout d'aînés et de cadets, quelles en seraient les conséquences génétiques, psychologiques et sociales ? Je l'ignore. Peut-être seraient-elles considérables. Quoi qu'il en soit, j'attire l'attention sur le fait que, si notre discussion doit se limiter plus ou moins à une comparaison entre la condition de la femme mariée dans la société primitive et dans la nôtre, cela implique une comparaison plus approfondie entre la famille primitive entendue comme un tout et la famille dans notre société. L'une des différences les plus évidentes entre elles réside dans le rôle des enfants, et en conséquence dans l'attitude procréatrice des parents.

Si notre étranger séjourne au milieu d'un peuple primitif pendant longtemps, en apprend la langue, participe à sa vie quotidienne, il découvrira rapidement que bien que l'amour sexuel s'y manifeste à profusion, il existe rarement un sentiment correspondant à ce que nous entendons par amour romantique. Quelques indigènes d'Afrique du Sud se plaignirent devant une commission gouvernementale que l'amour avait été introduit chez eux par les Européens. Nous sommes maintenant conditionnés, bien qu'il nous ait fallu des siècles, par la poésie, le théâtre, le roman, les journaux, le cinéma, la radio, la télévision et la publicité, pour admettre que l'amour romantique doit précéder le mariage, qu'il le précède en fait, et qu'il en est la seule justification. La jeune primitive, bien qu'elle ait naturellement des préférences, comprendrait difficilement l'amour sentimental et son rôle dans le mariage. De notre point de vue, il se peut qu'elle se prive d'un raffinement de la vie anglaise moderne, mais elle n'en a pas conscience, et, en outre, elle se marie sans illusion: elle n'attend rien d'autre que ce qui sera, et risque donc moins les déceptions. Il en va de même de l'homme qui, en l'absence de ce qu'on appelle «le mythe de la femme», se marie avec un être humain et non sa représentation idéalisée.

J'ai remarqué qu'il était impossible de comparer convenablement la condition de la femme dans les sociétés primitives avec sa condition chez nous, sans considérer l'ensemble des relations familiales dans les deux cas. Cependant, une comparaison entre la famille primitive et la famille anglaise d'aujourd'hui réclamerait un travail considérable, et je ne puis que mettre en évidence un aspect important de leur différence et suggérer une des causes de cette différence. Dire que les relations conjugales sont fortement influencées par ce qu'ont été les relations entre enfants et parents devient un lien commun. A mon avis, Malinowski avait donc certainement raison de critiquer les psychanalystes qui ne prenaient pas suffisamment en considération le fait que leurs conclusions se fondaient uniquement sur l'étude de sujets élevés dans un milieu familial tout à fait différent de celui que l'on trouvait dans les autres parties du monde et même parmi d'autres strates sociales en Europe. Il est vrai qu'il testait leurs découvertes sur un peuple, les Trobriandais, à descendance, héritage et résidence 
matrilinéaires. Mais chez les peuples primitifs patrilinéaires, les habitudes diffèrent aussi des nôtres, d'une manière significative.

Il suffit peut-être d'attirer l'attention sur une seule différence majeure : celle des relations mère-fils et père-fille. Aussitôt qu'un garçon sort de l'enfance, il ne subit plus que la seule autorité paternelle. Sa mère cesse d'avoir son mot à dire dans son éducation et d'exercer sur lui une réelle influence. D'autre part, la fille vit sous le contrôle presque exclusif de sa mère, et, en dehors des accords prénuptiaux, son père s'intéresse peu à ses affaires. En conséquence, on ne rencontre pas dans la famille primitive de mères trop possessives à l'égard de leurs fils, ni de filles trop sentimentalement attachées à leurs pères. Les diverses inadaptations qui pourraient en découler sont pratiquement absentes de la famille primitive.

Ces inadaptations que j'évoque, si évidentes dans notre société et plus encore aux ÉtatsUnis, vous sont devenues familières par la littérature psychologique et psychiatrique, et de tels sujets conviennent peut-être mieux à la discussion professionnelle que publique. Il me semble cependant que je dois mentionner l'une d'entre elles, l'homosexualité masculine, parce qu'elle concerne le thème de notre débat, également parce que, si l'on en croit tous les rapports et les études, elle a pris des proportions très inquiétantes, voire alarmantes. Le psychiatre américain Kardiner la décrivait comme une "répulsion massive du mâle pour la femelle »; et la plupart des psychiatres semblent s'accorder sur l'un des facteurs déterminants de ce phénomène : la domination de la mère et par conséquent la soumission du père. Ce symptôme d'une profonde dysnomie sociale est totalement absent des sociétés primitives. Lorsqu'on le rencontre, il constitue un fait exceptionnel ou un expédient passager, ou bien il joue un rôle culturel et par suite ne pose pas de problème social.

Il convient aussi de garder en mémoire le manque relatif d'intensité dans les relations familiales parmi les peuples primitifs, phénomène sans aucun doute en liaison avec un système de parenté. Nos familles vivent en petites communautés isolées. Les liens de parenté en dehors de la cellule familiale sont faibles, et notre société mobile nous sépare. On sait fort bien que, dans les sociétés primitives, il est un large éventail de personnes qu'un enfant appelle « père », « mère », « frère », « sœur », qu'il considère comme tels, et envers qui il agit comme tels. Ces personnes vivent généralement dans son entourage immédiat, si bien que, depuis son enfance, il les côtoie tous les jours et apprend à les traiter comme membres de sa famille au sens large. En conséquence, les sentiments et intérêts intrafamiliaux s'étendent à un très grand nombre de parents. Les primitifs n'ont pas notre sentiment écrasant de l'exclusivité familiale - la famille étant ce «nous » opposé au reste de la société : « eux». La société est pour eux, en grande partie, une extension des relations familiales. Et ces relations ne sont pas comme chez nous isolées par des cloisons étanches. Dans les sociétés les plus primitives, chaque foyer déborde sur l'autre et leurs membres se mêlent dans une vie commune, d'où l'intimité autant que le désir d'intimité sont absents. Il n'existe donc pas la même concentration émotionnelle que dans un minuscule cercle de personnes. On s'en aperçoit plus spécialement à la mort du père ou de la mère de famille. Leur fils ou leur fille peut être profondément affecté par cette perte, mais sans comparaison possible avec notre propre douleur.

Une autre caractéristique des sociétés primitives, ainsi que des sociétés orientales, qui frappe fortement un Anglais, c'est la non-ingérence mutuelle dans les affaires de l'autre sexe, contrairement à ce qui semble se passer chez nous, notamment entre époux. Je suis 
constamment tenu de parler d'époux, car la femme primitive est épouse. A la maison, le couple marié a sa vie de famille avec ses enfants, mais hors de la maison, le mari et, à un moindre degré, la femme mènent une vie sociale indépendante. Lui s'occupe de ses affaires, elle des siennes, sans souci du partenaire qui n'est pas concerné. Le mari vaque aux affaires publiques, mais aussi à sa vie sociale sans emmener sa femme avec lui. La femme mène sa propre vie sociale sans la présence constante de son mari. Là encore, avantages et inconvénients se compensent. Le sens de l'amitié entre homme et femme et spécialement entre mari et femme n'est pas aussi fort que dans notre société. Mais il en résulte moins de confusion entre les personnalités, une perte moins grande de cette qualité distinctive des sexes issue en partie d'une différenciation sociale, et à certains égards d'une spécificité sociale.

Une des raisons de la non-ingérence mutuelle dans les affaires du partenaire provient d'une division du travail entre les sexes, plus nette que chez nous. La confusion des rôles ne s'est développée que très récemment dans la société anglaise, car il y a peu de temps encore, une femme mariée se consacrait en général à sa famille et à ses enfants ; son mari participait aux affaires publiques et, selon les besoins, gagnait sa vie et celle de sa famille hors du foyer. C'est ce qui se passe dans la communauté primitive. Les hommes, et non les femmes, participent aux conseils, à la guerre, aux rites religieux, aux procédures judiciaires, etc., et leur contribution à l'économie familiale provient de tâches qui requièrent non seulement de la force mais aussi l'éloignement $\mathrm{du}$ foyer. La femme, elle, est assujettie aux corvées quotidiennes de la maison : préparation des repas, ménage, soin des enfants. Il paraît évident, lorsque nous comparons son sort à celui de la femme moderne, que le progrès technique et économique dans notre civilisation a permis à cette dernière de participer à un éventail plus large d'activités et d'acquérir ainsi une expérience sociale plus riche, ce qui a eu pour conséquence notoire d'effacer les différenciations sociales entre sexes, sans qu'il en résulte nécessairement un avantage pour la femme. Dans la société primitive, les sphères d'activité de chacun des sexes sont clairement délimitées : si la femme demeure en dehors des activités masculines, l'homme ne cherche pas à rivaliser avec elle dans les activités féminines, et ne s'immisce pas dans les problèmes domestiques traditionnellement du ressort féminin. Si cette limitation constitue une restriction pour la femme primitive, elle lui assure aussi une garantie.

Ceci me conduit au problème de l'autorité à l'intérieur de la famille. Dans les sociétés primitives, l'homme détient invariablement l'autorité, bien que dans certaines sociétés et dans certaines circonstances les vieilles femmes puissent aussi l'exercer. Comme vous le savez, il est des sociétés dans lesquelles l'héritage et la succession s'opèrent par la lignée utérine. Mais dans de telles sociétés, la femme, dans la mesure où elle échappe à l'autorité paternelle ou maritale, subit celle de son oncle maternel ou de son propre frère. Les sociétés matrilinéaires, moins nombreuses cependant que les sociétés patrilinéaires, se rencontrent à l'état le plus typique parmi des peuples comme les Nayars de la côte Malabar de l'Inde et les Manangkabau de Sumatra, dont les cultures sont bien loin d'être primitives. Mais on ne les rencontre pas au sein des grandes civilisations du monde, toutes patriarcales. Pour éviter une discussion sur le problème quelque peu particulier de l'autorité dans les sociétés de type matriarcal, je ne ferai état que des sociétés primitives les plus courantes dans lesquelles, à son mariage, la femme échappe à l'autorité paternelle pour subir l'autorité maritale. En fait, la coutume et les conventions limitent l'autorité du mari. Sa femme possède bien des moyens de l'influencer, d'obtenir gain de cause, et la plupart du temps elle y réussit. Mais l'autorité du mari comme telle n'est pas mise en cause. Ni la femme, ni qui que ce soit, ne conteste que 
c'est à lui, et à lui seul, de prendre les décisions importantes en ce qui concerne la maison, l'éducation des enfants, les fiançailles des filles et des fils, etc. Dans l'Angleterre d'aujourd'hui, il est indéniable que l'homme a perdu la plus grande part de l'autorité dont il jouissait en tant que mari et père. En réalité, je crois possible de dire qu'il a pratiquement perdu sa position de chef de famille et que souvent il tend à adopter dans son foyer une attitude défensive ou même passive.

Mais la condition de la femme anglaise moderne ne s'est-elle modifiée qu'à son avantage ? Je me le demande. Je ne suggère pas que l'autorité incontestée du mari, dans les sociétés primitives, évite toutes les controverses, disputes et autres désagréments domestiques. Loin de là. D'une certaine façon, ils surgissent de la nature même de la famille en tant qu'institution. Mais je les crois plus superficiels et plus limités, dès lors qu'ils ne mettent pas fondamentalement en cause la position de l'homme en son foyer et ne touchent pas, comme chez nous, à presque toutes les sphères d'activités dans lesquelles le couple ou l'un de ses membres est engagé. Les possibles désaccords n'affectent pas la vie sociale de la famille tout entière, évitant ainsi des souffrances à la femme, aussi bien qu'au mari et aux enfants.

A la base de toutes les différences que j'ai notées, il en existe une fondamentale entre l'attitude de la femme primitive et celle d'un grand nombre de femmes anglaises modernes. Il est difficile de la décrire en quelques mots, mais on pourrait la résumer de la sorte : la femme primitive, si l'on ne respecte pas ses droits et privilèges, se plaint publiquement et rend la vie impossible à son mari et à tous, mais elle ne ressent pas le désir d'un changement de statut. En considérant sa condition sociale de l'extérieur et de notre point de vue, on peut dire qu'elle vit en état d'infériorité, qu'elle le sent peut-être, mais qu'elle n'en éprouve aucun ressentiment. Elle s'estime différente de l'homme et jouissant, par conséquent, d'un statut social différent. Pour définir son attitude dans notre propre langage, nous suggérerions qu'il s'agit moins pour elle d'un problème de niveau que d'un problème de différence de statut. La femme primitive ne se considère pas comme appartenant à une classe défavorisée, face à la classe des hommes dont elle voudrait devenir socialement l'égale. Elle n'a jamais entendu parler d'égalité sociale et ne souhaite pas non plus ressembler à l'homme. Dans notre propre société, le problème n'est pas aussi simple, parce que la condition de la femme, objet de spéculation philosophique aux XVIIIe et XIXe siècles, s'est trouvée mêlée à toutes sortes de mouvements libéraux et égalitaires, d'où sont nées les idées d'assujettissement de la femme et d'émancipation auxquelles J. S. Mill, dans son fameux essai de 1869, donna une grande impulsion, et qui furent, plus tard, énergiquement défendues par le mouvement féministe.

J'ai fourni quelques exemples - modestes, mais j'espère peu communs - de ce que je considère comme des différences entre la femme (ou plutôt l'épouse) anglaise moderne et sa sœur primitive, et je conclurai maintenant mon exposé par quelques considérations générales qui résultent de notre débat. Sans doute avez-vous été frappés de ce que la plupart des différences que j'ai notées - et j'ai dû opérer un choix - sont des différences que nous remarquons, non seulement quand nous comparons notre propre société contemporaine avec les sociétés primitives, mais aussi quand nous la comparons avec la société anglaise, notamment celle de l'Angleterre rurale d'avant la révolution industrielle. A cette époque, les parents, comme dans les sociétés primitives, ne limitaient pas le nombre de leurs enfants, on ne considérait pas l'amour romantique comme l'unique raison du mariage, les liens de parenté jouaient un rôle plus important, les femmes se préoccupaient (à quelques exceptions près 
dans les villes, car l'industrialisation est un phénomène relatif) presque exclusivement des problèmes domestiques, l'homme était le chef de famille, et ni sa femme, ni ses enfants ne contestaient son autorité, bien qu'ils la bravassent parfois dans la mesure où ils mettaient en cause son exercice. En réalité, quand nous lisons les récits et les romans des périodes antérieures de notre histoire, nous nous apercevons qu'en ce qui concerne la grande masse de la population, la condition de la femme, dans la société et dans son foyer, était, pour l'essentiel certainement, semblable à celle de la femme dans les sociétés primitives. Les changements qui se sont produits dans le statut de la femme, et qui ont conduit aux différences que nous avons remarquées entre les sociétés primitives et la nôtre, doivent donc être rattachés aux grandes révolutions techniques et sociales du siècle dernier. En conséquence, ce qu'il nous faut opposer, ce n'est pas tant le statut de la femme dans les sociétés civilisées et son statut dans les sociétés primitives, que son statut dans une société fortement industrialisée et son statut dans une société préindustrielle. Il n'est donc pas possible de traiter de ce qu'on appelle le progrès de la femme au siècle dernier, sans tenir compte de l'ensemble des changements intervenus à cette époque-là : industrialisation, urbanisation, mécanisation, nationalisation, laïcisation et bien d'autres révolutions sociales. Comment ces vastes mouvements ont-ils affecté la vie de la femme est un sujet fort complexe qui attend encore qu'un historien-sociologue s'y intéresse. Tout ce que je puis vous proposer maintenant se limite à quelques observations générales et sommaires dans le cadre restreint de notre recherche.

Lorsque nous nous demandons si les bouleversements sociaux ont été favorables à la condition de la femme, nous posons là une question à laquelle il est presque impossible de répondre, étant donné le nombre de facteurs en jeu, la difficulté de les déterminer quantitativement et l'absence de critères fixes à partir desquels nous pourrions porter un jugement. Néanmoins, une personne qui a vécu longtemps parmi nombre de peuples primitifs peut détourner son esprit de nos conditions sociales pour le diriger vers les leurs et parvenir à une comparaison. Ce faisant, je songe à certaine caractéristique de la vie primitive qui nous apparaît désavantageuse pour la femme en tant que telle, et par référence avec sa condition dans notre société. Mais je pense aussi à certains inconvénients qui accompagnent l'amélioration de cette condition et qui constituent le prix dont il la faut payer. Au cours de cette conférence, j'en ai cité quelques exemples : l'impossibilité de se marier, l'inadaptation psychologique dans la vie familiale, qui, en l'absence d'étroites relations de parenté, se trouve porteuse d'une charge émotionnelle trop lourde, l'incertitude quant au statut, avec les confusions et les frictions qui en découlent, l'ennui, etc. Faut-il y ajouter la pudibonderie et la prostitution décrite avec tant de complaisance par Lecky comme étant « en définitive le plus efficace garant de la vertu ».

Si l'on conserve présente à l'esprit l'idée que pour chacune des frustrations dont elle souffre, la femme primitive reçoit des compensations auxquelles la femme, chez nous, ne peut prétendre, on parvient à la conclusion suivante : tout bien considéré et pesé, on ne saurait affirmer, sans de grandes réserves, que la femme soit beaucoup plus favorisée dans notre société que dans les sociétés primitives. De peur que vous ne considériez cette affirmation comme extravagante ou même absurde, je m'empresse de redire que je parle de la condition de la femme par rapport à celle de l'homme. Manifestement, la femme anglaise jouit de facilités, de loisirs, de luxes, profite des avantages du christianisme, de l'éducation, de la médecine, de la mécanisation, en fait de tout ce que nous appelons civilisation et qui fait défaut à la femme primitive ; mais il en va de même pour l'homme. En réalité, on peut 
raisonnablement soutenir que, si la femme moderne est remarquablement mieux nantie que la femme primitive, cela ne découle en rien d'un changement de son statut dans la société, c'està-dire vis-à-vis de l'homme, mais d'innovations qui ont également affecté homme et femme. Le statut de la femme demeure relativement stable pour l'essentiel et en dépit de toutes les apparences formelles et conventionnelles. Ceci admis, on peut supposer que certains changements intervenus chez nous dans la situation de la femme, et qui contrastent avec les conditions primitives, se révéleront transitoires, particulièrement ceux de date récente, survenus dans des circonstances historiques très particulières.

J'irai même plus loin. Il me semble difficile de croire que la condition relative des sexes puisse subir d'importantes et durables modifications dans un proche avenir. Les sociétés primitives, les sociétés barbares, les sociétés historiques d'Europe et d'Asie offrent presque toute la gamme des institutions concevables. Mais dans toutes ces sociétés, abstraction faite de la forme des structures sociales, l'homme conserve l'ascendant. Ceci est peut-être d'autant plus évident que le degré de civilisation est plus élevé. Autant que j'en puisse juger et sans vouloir être provocant, c'est un fait. Peut-être en serait-il mieux autrement. Les féministes en donnent pour raison qu'on a toujours refusé aux femmes l'occasion de prendre le commandement. Nous pouvons alors nous demander comment elles ont accepté que l'occasion ne leur en soit jamais offerte; la seule force brutale ne peut suffire à en rendre raison. Les faits semblent plutôt suggérer que des facteurs biologiques et psychologiques profonds, aussi bien que des facteurs sociologiques, interviennent, que les relations entre les sexes peuvent être seulement modifiées par des changements sociaux et non pas radicalement transformées (ce qui, si je comprends bien, est la conclusion de Lecky). Il est indubitablement avéré que dans toutes les sociétés les petites filles et les adolescentes se modèlent, éventuellement avec l'aide de leurs aînées, sur l'image de la femme dans leur société ; mais soutenir avec certains écrivains, Margaret Mead par exemple, et Simone de Beauvoir, dans son remarquable ouvrage: Le deuxième sexe (1949), que les différences sociales et les différences de tempérament entre les sexes ne sont que le produit d'un conditionnement culturel, est une réification qui n'explique rien, et certes pas un trait aussi universel que le leadership masculin.

Je suggérerais que, en dépit des décrets officiels, ce qui a changé, c'est moins la condition relative des sexes dans la société, que le statut, au sens le plus large du terme, de l'individu, homme ou femme ; et je soutiendrais que la condition de la femme dépend en définitive de notre condition commune : nous subsistons ou disparaissons ensemble. Ce point de vue ne correspond certes pas à celui de la plupart des premières féministes - leur cause en était d'autant affaiblie - qui ne menaient leur lutte que contre la ségrégation des sexes et la domination masculine. Il eût mieux valu la présenter, comme d'autres le firent, comme un aspect du combat général pour l'obtention des droits sociaux fondamentaux et de la dignité de tous les êtres humains : homme ou femme, adulte ou enfant, riche ou pauvre, bien portant ou malade, noir ou blanc. Le problème du statut de la femme perd sa signification face à celui-ci plus général et plus fondamental.

Une étude des sociétés primitives et des sociétés de culture plus avancée confirme mon opinion, valable au moins en tant qu'a priori. Je ne saurais citer ici toutes les preuves tirées des sociétés primitives, mais je puis affirmer, en m'appuyant à la fois sur mes propres recherches et sur mes lectures, que dans ces sociétés où une fraction de la population vit dans la servilité, la condition de la femme, spécialement dans la vie conjugale, est 
proportionnellement inférieure à celle de l'homme. Ce fait apparaît souvent avec évidence dans les sociétés où la masse de la population est assujettie à une classe dirigeante qui l'exploite. Selon la remarque pertinente de Montesquieu, deux choses sont étroitement liées : le pouvoir despotique du prince entraîne naturellement la servitude de la femme ; l'esprit de la monarchie, la liberté de la femme. Encore faut-il saisir, évidemment, ce qu'il entend par despotisme et monarchie.

En dernier lieu, le statut de la femme, en particulier son statut dans le foyer, dépasse le champ de l'analyse sociologique. C'est au fond une question morale, et s'il appert qu'on doive considérer les découvertes de la recherche sociologique pour porter un jugement, le jugement lui-même doit découler de quelque code moral. Après tout, le mariage n'est pas une simple relation entre l'homme et la femme en tant que mâle et femelle, c'est beaucoup plus l'adaptation mutuelle d'individus dans une certaine relation sociale... Les difficultés qui surgissent tiennent à la situation elle-même. Elles ne surviennent pas seulement entre époux ou entre membres de sexes opposés, mais aussi entre parents et enfants, entre frères et sœurs, professeurs et élèves, patrons et employés, citoyens d'un pays et d'un autre, etc. Dans la mesure où les rapports entre sexes ne sont pas seulement des relations sexuelles, mais d'autorité, de leadership, de contrôle, de coopération et de concurrence, des problèmes apparaissent dans chaque secteur social et dans chaque société. L'acceptation des différences sociales, une certaine indulgence et la reconnaissance de l'autorité résolvent mieux ces problèmes que les revendications d'égalité absolue, sinon les antagonismes deviennent inévitables et une vie sociale paisible et harmonieuse impossible. La reconnaissance de l'autorité, loin d'entraîner une infériorité, exprime la seule vraie forme d'égalité entre les hommes : une utilité sociale identique.

Tous ceux dont les opinions méritent considération reconnaissent que la valeur d'une personne ne peut être jugée sur sa seule place dans la société, mais doit l'être d'après beaucoup d'autres critères. L'estime accordée à un individu ne découle pas seulement de son statut social, de sa richesse, de son éducation, de ses chances, de ses qualifications occasionnelles, mais aussi et surtout de ses qualités intellectuelles, de son tempérament et de sa sagesse. De telles qualités n'ont rien à voir avec le sexe d'une personne, ni avec le statut social des sexes, quelque importance que celui-ci puisse avoir.

Pour conclure, je veux seulement ajouter que, malgré les insuffisances de cette conférence, dont je suis le premier à reconnaître les déficiences, je la crois plus pertinente que beaucoup de discussions sur la condition féminine. J'ai beaucoup lu pour préparer cette conférence, mais sans réel profit, je dois l'avouer. Ce qui m'a le plus frappé au cours de mes lectures, ce n'est pas tant telle observation ou telle idée, que l'insuffisance et l'absence presque totale de recherche scientifique sérieuse sur les questions que nous avons abordées, aussi bien dans les sociétés primitives que dans la nôtre. J'irai jusqu'à souhaiter, non seulement que cette recherche soit menée à bien, mais qu'elle soit effectuée sous la direction de ce collège auquel j'ai l'honneur de m'adresser, et au nom de l'illustre dame dont nous honorons la mémoire. 


\section{BIBLIOGRAPHIE \\ du chapitre 2}

$\underline{\text { Retour à la table des matières }}$

COURTNEY (Janet E.), The Adventurous Thirties, 1933.

CRAIK (Mrs), A Woman's Thoughts about Women, N.D. FAWCETT (Millicent Garrett), What I Remember, 1924. HARTLEY (C. Gasquoine), The Position of Women in Primitive Society, 1914. HOBHOUSE (L. T.), Morals in Evolution, 2 vol., 1906.

JAMES (B. B.), Women of England, 1907-1908.

KABERRY (Phyllis M.), Aboriginal Woman, 1939. - Women of the Grassfields, 1952.

KARDINER (Abram), Sex and Morality, 1955.

LECKY (W. E. H.), History of European Morals, 1924 éd. (1re éd., 1869). LOWIE (Robert H.), Primitive Society, 1929 éd.

— The Crow Indians, 1935.

MALINOWSKI (B.), Sex and Repression in Savage Society, 1927. MASON (0. T.), Woman's Share in Primitive Culture, 1895.

McGREGOR (0. R.), The Social Position of Women in England, 1850-1914; a Bibliography, The British Journal of Sociology, 1955.

MEAD (Margaret), Sex and Temperament, 1935.

MEAKIN (A. M. B.), Women in Transition, 1907. MILL (J. S.), The Subjection of Women, 1869.

MILLAR (John), The Origin of the Distinction of Ranks or an Inquiry into the Circumstances which give rise to Influence and Authority in the different Members of Society, $4 \mathrm{e}$ éd., 1806 (1re éd., 1771).

MONTESQUIEU (Charles-Louis de Secondat, baron de), L'esprit des lois, 1748. NIEBOER (H. J.), Slavery as an Industrial System, 1910 éd. (ire éd., 1900). 
PLOSS (H. H.), BARTELS (M.) et BARTELS (P.), Woman, An Historical Gynaecological and Anthropological Compendium, édit. par E. J. DINGWALL, 1935 (ire éd. allemande, 1885).

SPENCER (Herbert), The Principles of Sociology, 2 vol., 1882, 1883. WEST (D. J.), Homosexualily, 1955.

WESTERMARCK (Edward), The History of Human Marriage, 3 vol., 1921 (1re éd., 1891).

The Origin and Development of the Moral Ideas, 2 vol., 1906. WIETH-KNUDSEN (K. A.), Feminism, trad. anglaise, 1928.

WOOLF (Virginia), Three Guineas, 1938.

YOUNG (G. M.), Victorian England, Portrait of an Age, 1936. 


\section{"Structures politiques des peuples de langue Nandi du Kenya" * (1940)}

Cet article se propose d'analyser brièvement la littérature sur les peuples de langue nandi du Kenya afin de dégager entre eux des similitudes et des différences de structure. Plus précisément, 'Cette recherche vise à découvrir si leurs structures politiques appartiennent au même type.

Le groupe de langue nandi, parmi les peuples du Kenya, comprend : les Nandi, les Keyu (Elgeyu), les Tuken (Kamasya), les Kipsigis (Lumbwa), les Terik (Nyangori), les Kony (Elgonyi), les Pok (Lako), les Suk (Pokwut), les Endo, les Marakwet et les petites communautés de chasseurs Okiek (Dorobo) ${ }^{1}$. Ces peuples parlent des dialectes d'une même langue et, à l'exception des Okiek, se ressemblent beaucoup des points de vue physique et culturel. On observe chez tous, sauf chez les Suk, pasteurs nomades des plaines, et dans les communautés de chasseurs, un même type d'environnement, un même genre de vie

Texte paru pour la première fois dans Africa, 1940. Mme Marion CARR, MM. A. R. RADCLIFFEBROWN, J. H. DRIBERG et J. G. PERISTIANY en révisèrent les épreuves.

1 Carte, p. 62, préparée par M. G. W. B. HUNTINGFORD. 
économique et un même intérêt pour l'élevage. Dans quelle mesure peut-on leur reconnaître des structures sociales similaires, le problème vaut la peine d'être étudié. Le livre que Peristiany ${ }^{1}$ a consacré aux Kipsigis nous fournit, pour la première fois, une étude scientifique portant sur un peuple de langue nandi, à partir duquel nous pouvons établir des comparaisons. La littérature, plutôt médiocre, sur les autres membres du groupe, manque de précision ${ }^{2}$. Seule celle sur les Nandi, les Suk et les Keyu peut supporter l'analyse.

J'emploie le passé lorsque je me réfère aux témoignages des spécialistes. Les organisations politiques des peuples du Kenya ont subi des changements depuis qu'ils ont écrit, et, assez souvent d'ailleurs, ils parlaient d'institutions qui, déjà au moment de leurs recherches, avaient, au moins partiellement, cessé de fonctionner.

Le mode de distribution écologique semble avoir été généralement le même parmi les Kipsigis, les Nandi, les Suk et les Keyu. Ils ne vivaient pas dans des villages, au sens d'agglomérations, mais dans des habitations isolées, accrochées aux flancs des collines,

Quant à l'organisation territoriale, elle présentait de nombreuses ressemblances chez ces quatre peuples. Chacun de leurs pays se divisait en secteurs territoriaux que nous pouvons appeler provinces, qui constituaient non seulement des divisions géographiques mais aussi des unités politiques. Chez les Kipsigis, il existait quatre provinces (emet, pl. emotinwek). Chacune d'entre elles avait ses propres organisations judiciaires et militaires, ses propres rites de récolte, ses propres cérémonies de changement de classe d'âge. Chez les Nandi, divisés en quinze provinces (pororiet, $\mathbf{p l}$. pororosiek), chacune opérait ses propres razzias. Bien que nos sources restent assez vagues, il existait probablement quelque organisation judiciaire basée sur le district (partie de province). Chaque province avait ses propres fêtes des récoltes, et selon Huntingford, ses propres cérémonies d'initiation. Les Suk se répartissaient aussi en secteurs territoriaux. Il est fort vraisemblable que ces provinces n'offraient pas la même netteté politique, ni la même cohésion, que chez les Kipsigis et les Nandi, mais elles revêtaient probablement une signification politique plus grande que Beech et Barton ne le suggèrent. Dundas remarque que chaque district possédait des droits de chasse sur son propre territoire et conduisait son initiation indépendamment des autres. Beech fait état de huit secteurs, Barton également, mais qui ne se recoupent pas exactement chez les deux auteurs. Dundas parle de onze, mais il est difficile de le suivre sur ce point. Autant qu'on en puisse juger, ces secteurs territoriaux ressemblaient fort aux provinces Nandi et Kipsigis. Chez les Keyu, Massam distingue seize petits secteurs politiques, parfois en guerre les uns contre les autres. Les différents districts de secteurs conduisaient séparément leurs rites d'initiation et leurs cérémonies de changement de classe d'âge.

J. G. PERISTIANY, The Social Institutions of the Kipsigis, 1939.

2 Principaux ouvrages et articles sur les Nandi: A. C. HOLLIS, The Nandi, their language and Folklore, 1909; Charles HEMSTED, The Tribal Organisation of the Nandi, J.E.A.U.N.H.S., 1923; G. W. B. HUNTINGFORD, The Nandi Pororiet, J.R.A.I., 1935, et Miscellaneous Records relating to the Nandi and Kony Tribes, ibid., 1927. Sur les Keyu : J. A. MASSAM, The Cliff Dwellers of Kenya, 1927; et sur les Suk: M. W. H. BEECH, The Suk, their Language and Folklore, 1911 ; K. R. DUNDAS, Notes on the Tribes inhabiting the Baringo District, East Africa Protectorate, J.R.A.I., 1910; Juxon BARTON, Notes on the Suk Tribe of Kenia Colony, ibid., 1921. Les quelques références relatives aux autres membres du groupe ne méritent pas d'être citées. 
Le point le plus faible des récits sur les Nandi, les Keyu et les Suk réside dans l'explication de l'organisation territoriale de ces peuples. Après nous avoir présenté une division en secteurs que nous avons appelés provinces, on nous indique que ces secteurs se subdivisaient eux-mêmes en sous-secteurs, mais sans nous signaler la nature de ces soussecteurs, ni leur rôle dans l'ensemble du système territorial. Il nous faut deviner quelle était l'organisation d'une province et les règles qui présidaient aux relations de ses différentes parties. Le livre de Peristiany sur les Kipsigis nous permet de hasarder une hypothèse.

Peristiany explique que plusieurs maisons voisines formaient une unité sociale distincte, le hameau (temet), où un ancien exerçait une certaine fonction judiciaire. Plusieurs hameaux constituaient un village disséminé (kokwet). Ces villages relativement peu éloignés n'en étaient pas moins des unités sociales très distinctes. Chacun possédait un chef judiciaire (kiruogindet), un leader économique chargé des problèmes d'assistance mutuelle pour le travail agricole du village, et un chef guerrier organisant la défense. Le village était l'unité de base du système territorial. Plusieurs villages formaient ce que Peristiany nomme un « groupe », que nous pouvons appeler district qui se trouvait sous l'autorité, judiciairement, du grand juge de district (kiruogindet neo), militairement, d'un chef guerrier, rituellement, d'un maître des cérémonies. Les districts se groupaient en provinces dont nous avons déjà parlé. Chez les Kipsigis, la province était le groupe organisé le plus important, mais les lois s'étendaient à toutes les provinces qui se coalisaient parfois pour se défendre. Le peuple Kipsigis n'avait donc pas en tant que totalité de système politique organisé. On peut dire qu'il appartenait, à certains égards, à un système politique inorganisé plus vaste, qui comprenait les provinces nandi avoisinantes. Point n'est besoin d'approfondir ici cette question.

Les Nandi jouissaient très probablement d'une organisation similaire, mais nous ne saurions l'affirmer, vu la pauvreté de la littérature qui les concerne. Hemsted et Huntingford parlent de paroisses (koret, pl. korotinuek) et Hemsted précise que chaque koret possédait son kokwet, arbre sous lequel se réunissaient les membres de la paroisse présidés par un ancien (boyot). Il prétend qu'une remarquable cohésion sociale régnait dans ce groupe. Il est probable que nous sommes ici en présence de l'organisation d'un kokwet kipsigis, et que le boyot des Nandi exerçait une fonction semblable à celle du chef de village kipsigis (poyot $a b$ kokwet). HOLLIS fait mention d'une subdivision de la province, sorte de paroisse (siritiet, pl. siritaiik) qui groupait trente à cinquante guerriers sous la direction d'un olaitoriot responsable devant le kiruogindet de la province. Selon lui, il s'agissait d'une unité administrative de la province. Hemsted parle d'un siritiet comme d'une milice de district, et Huntingford comme d'un petit groupe de guerriers. Le mot s'employait peut-être pour décrire l'organisation militaire d'un groupe local appartenant à la province. Comme chez les Kipsigis, bien que la province nandi ait été le groupe politique organisé le plus vaste, les lois s'appliquaient à tout le pays.

Quant aux Suk et aux Keyu, nous savons seulement que leurs provinces comportaient des subdivisions, dont nous ignorons la signification politique. Il est cependant clair que leur organisation territoriale ressemblait à celle des Kipsigis.

Peristiany eut peut-être tendance à trop formaliser le système territorial des Kipsigis, et à trop le segmenter. Mais il n'est pas douteux que de nombreux groupes existaient, non seulement chez les Kipsigis, mais parmi les autres tribus de langue nandi. Les méthodes d'enquête qu'utilisèrent les observateurs inexpérimentés ne leur permirent pas de dégager une 
organisation territoriale, notamment par absence d'une délimitation géographique précise des groupes.

Nous savons maintenant que l'organisation judiciaire des Kipsigis se fondait sur l'organisation territoriale. Nous ne nous étonnerons donc point de n'être pas parvenus à éclaircir le cas des autres peuples, puisque leur organisation territoriale n'a pu être définie. Là encore, il se peut que Peristiany ait présenté une image trop formelle de la hiérarchie judiciaire chez les Kipsigis. Néanmoins, il a montré l'existence d'une organisation judiciaire basée sur les structures territoriales. Il est évident, d'après les sources que nous possédons, que d'autres peuples avaient une semblable organisation, même si elle n'a pas été perçue.

Peristiany décrit comment la cause qu'on ne pouvait résoudre au hameau était portée devant le juge du village qu'aidaient les anciens des hameaux constitutifs. Si elle ne pouvait être résolue par l'autorité du village, on la portait devant le juge du district assisté des délégués des villages constitutifs. En cas de différend entre des individus de divers districts, l'affaire se réglait devant une cour formée des juges de districts et de leurs assistants.

Nous ne disposons d'aucune information précise sur le système judiciaire nandi. HOLLIS suppose qu'il n'en existait aucun et que les parties concernées réglaient leurs différends par des négociations directes. Il note cependant que les dirigeants réels des provinces étaient ce qu'il appelle des «conseillers» (kiruogindet, pl. kiruogik), bien qu'en fait ils aient été responsables de l'ordre et de l'organisation militaire devant le prophète (orkoiyot), à travers ses représentants. Un olaitoriot responsable devant le kiruogindet était à la tête de chaque paroisse. L'auteur décrit donc une sorte d'organisation administrative, mais il est probable qu'il confond les systèmes judiciaire et militaire, tous deux basés, chez les Kipsigis, sur des divisions locales. Hemsted parle d'un conseil des capitaines de toutes les classes d'âge (kiruogik), présidé par le kiruogindet ap boriet. D'après Huntingford, dans chaque province un conseil d'anciens exerçait l'autorité.

Certes, il serait hasardeux de présumer que l'organisation nandi ait été la réplique exacte de celle décrite par Peristiany chez les Kipsigis. Mais on peut supposer, sans aucun risque, que les kiruogik nandi équivalaient, dans l'ensemble, aux kiruogik kipsigis et que, si Peristiany a décrit correctement le système judiciaire des Kipsigis, un système semblable, à base locale, existait chez les Nandi.

Beech et Barton remarquent que les Suk n'avaient pas de «chefs » mais que chaque «village », terme par lequel ils désignent le hameau, avait à sa tête un homme âgé. Un certain nombre de «villages » se rassemblaient autour de l'un de ces hommes âgés (ki-ruwokin, pl. ki-ruwok-u), ou conseiller, qu'assistaient les anciens. Beech ajoute que son autorité était faible et sa fonction non héréditaire. Peut-être en était-il ainsi, mais dans sa description, nous discernons les grandes lignes d'une organisation qui devait, à bien des égards, ressembler à celle des Kipsigis. Massam note que les Keyu n'avaient pas de chefs, au sens habituel du terme. Là encore, nous trouvons le conseiller (ki-ruwek-in) choisi, comme chez les Suk, en fonction de ses aptitudes et de sa richesse. Ces conseillers présidaient, sous un arbre, des assemblées locales (ka-kiruok), des réunions d'anciens. Avec évidence, on reconnaît là le conseil d'anciens du village kipsigis (kapkiruok). 
Chez toutes ces tribus, un individu qu'on pourrait appeler «prophète» assumait d'importantes fonctions rituelles. Il célébrait certains rites magiques au nom de tout le peuple. Son rôle principal consistait à prédire le résultat des razzias et à exécuter des pratiques magiques pour en assurer le succès. Il ne jouait aucun rôle profane. Il symbolisait, peut-on dire, les sentiments d'unité entre les provinces politiques dont se composait un peuple, face aux communautés hostiles avoisinantes. L'incertitude de son statut, son manque d'influence dans le domaine profane, reflétaient bien l'inorganisation et l'incoordination entre les différentes provinces. Chez les Kipsigis, un tel prophète s'appelait laibon. Sa fonction principale consistait à prévoir le résultat des razzias en perspective. C'était l'homologue de l'orkoiyot ( $\mathrm{pl}$. orkoiik) héréditaire nandi qui prédisait le résultat des razzias et accomplissait des rites magiques pour leur succès. Il exerçait aussi certaines fonctions rituelles en rapport avec les semailles, la pluie, la fertilité des femmes et des animaux. Toutes les sources s'accordent sur son absence presque totale d'autorité profane. Beech remarque que, chez les Suk, personne ne jouissait, près du peuple tout entier, d'une telle réputation. Cependant, Barton mentionne un individu (wuregoion) chargé de prédire le résultat des razzias, d'effectuer des rites magiques pour leur succès, et qui était aussi maître des cérémonies en l'honneur du bétail. Ses fonctions apparaissent comme entièrement rituelles. Selon Massam, chez les Keyu, personne ne bénéficiait d'une estime comparable à celle de l'orkoiyot nandi, cependant, avant une razzia, on consultait un prophète (moiyet).

Le statut relatif de ces personnages sacrés semble être un indice de la cohésion entre les unités politiques qui composaient les différents peuples. Ainsi, ce statut était plus élevé chez les Nandi et les Kipsigis que chez les Suk et les Keyu, et de toute évidence un plus grand degré de cohésion régnait au sein des provinces nandi et kipsigis. C'est chez les Nandi que ce statut était probablement le plus élevé.

Au point de vue développement du système juridique, tous ces peuples se situent à peu près au même niveau. Peristiany note que, chez les Kipsigis, dans toutes les causes, du ressort civil pour la plupart, l'autodéfense soutenue par l'opinion publique constituait le mode principal de conciliation. Le règlement des querelles au niveau des cours d'arbitrage s'accompagnait de rites qui lui donnaient un caractère sacré. Le rôle des juges se limitait à celui de conseillers qui annonçaient officiellement les décisions prises avec l'accord général de toutes les parties en présence. Néanmoins, un système de châtiment punissait le malfaiteur récidiviste ou l'individu dangereux pour la communauté. Celle-ci pouvait le bannir publiquement et le mettre à mort ou le déclarer maudit, ce qui, croyait-on, entraînait sa mort.

Le même état de choses régnait chez les Nandi. Le combat constituait la seule réparation possible de l'adultère. La négociation directe entre les parties en cause réglait les querelles. La communauté punissait l'inceste et le vol. La différence principale entre les récits que nous possédons sur les Nandi et les Kipsigis est la suivante : tandis que Peristiany nous a décrit un système de cours pour le règlement des querelles, les sources dont nous disposons pour les Nandi ne font état d'aucune institution judiciaire ${ }^{1}$.

Il semble que, chez les Suk, la procédure adoptée pour le règlement des querelles ait été l'autodéfense appuyée sur l'opinion publique. Cependant aucun récit ne nous en fournit un

Hemsted parle d'un conseil réglant les querelles d'héritage chez les Nandi, mais aucun autre auteur n'en fait état. Nous avons de bonnes raisons de mettre en doute son assertion à ce sujet. 
compte rendu très clair. Beech signale que le jugement était prononcé en accord avec l'avis de la majorité des anciens et des guerriers. L'action collective punissait les crimes. Selon lui, chez les pasteurs suk, la communauté poussait les parents d'un sorcier à le mettre à mort, «à moins, ajoutait un informateur, qu'ils ne s'y refusent, auquel cas l'affaire était close ». Toujours d'après le même auteur, un sorcier récidiviste était mis à mort chez les agriculteurs suk. Parmi ceux-ci, il en allait de même pour le voleur récidiviste que, sous la pression communautaire, son propre frère devait tuer. Les récits de Beech suggèrent que les difficultés d'application des jugements étaient grandes. Beech, Dundas et Barton ne décrivent aucune procédure qui puisse relever, à proprement parler, d'institutions judiciaires.

Bien que les récits de Massam sur les Keyu relatent fréquemment des jugements prononcés par les anciens et les conseils, à propos de problèmes interindividuels, il est évident que de tels jugements se limitaient à exprimer l'opinion coutumière. Selon cet auteur, les lois relevaient toutes plus ou moins d'un droit civil. Cependant, la communauté punissait les malfaiteurs récidivistes, et, avec l'accord de la parenté, obligeait un voleur confirmé à se pendre. Elle sanctionnait également le sorcier si celui-ci nuisait régulièrement, en l'obligeant aussi à se pendre, avec le consentement de ses parents. Massam ajoute que, si un homme avait des rapports avec une femme mariée, les femmes de la communauté s'emparaient de ses biens et démolissaient sa case. Quiconque agissait de façon antisociale pouvait être banni par le conseil des anciens de la communauté.

Les Nandi, les Kipsigis, les Keyu et les Suk avaient le même système de classes d'âge, trait dominant, parmi d'autres, de leurs structures sociales. Par l'échelle des multiples classes, on accédait successivement du stade d'enfant à celui de guerrier puis à celui d'ancien. Chez les Nandi, on comptait sept classes (ipinda, pl. ipinuagik) de même que chez les Kipsigis (ipinda, pl. ipinwek). Les Keyu et les Suk (pen) en avaient huit ${ }^{1}$. Chez les Nandi, HOLLIS signale un intervalle de sept ans et demi entre le passage initiatique d'une classe à une autre. Hemsted parle de douze ans et Huntingford de quinze. Beech estime à environ quinze ans cet intervalle chez les Suk, tandis que pour Barton la périodicité n'est pas fixée. Chez les Kipsigis, Peristiany parle d'une période irrégulière de quinze à vingt ans. Il écrit qu'en règle générale les garçons d'une classe n'étaient pas initiés pour entrer dans la classe voisine mais dans la classe alterne (tel était probablement toujours le cas des fils aînés). Quelquefois ils sautaient même deux classes, mais ils n'étaient jamais initiés pour entrer dans la classe immédiatement supérieure. Chez les Keyu, Massam signale qu'il y avait toujours une classe intermédiaire entre celle du père et celle du fils. Il s'agit probablement de la règle appliquée ailleurs aux fils aînés. Parmi ces quatre peuples, le système de classes d'âge était cyclique. Chaque classe se stratifiait en de nombreuses divisions ou sous-classes. Chez les Nandi, les Kipsigis et les Suk, on en comptait trois. Chez les Keyu, il semble n'y en avoir eu que deux. Quand une nouvelle classe était initiée, et que par la circoncision elle accédait au stade de guerrier, tandis que les membres de ce groupe passaient à celui d'anciens, d'importantes cérémonies se déroulaient chez les Nandi, les Kipsigis et les Keyu. Selon Beech et Barton, les Suk ne connaissaient aucune cérémonie correspondante.

L'organisation militaire des provinces avait pour base, chez ces quatre peuples, les classes d'âge d'un district, et la coordination de l'action ne dépassait pas les circonscriptions locales. Chez les Kipsigis, l'organisation défensive se composait probablement de la totalité des

\footnotetext{
Barton mentionne douze classes chez les Suk, mais n'en dénombre que dix. Il les dit totémiques.
} 
effectifs d'une localité, divisés en sections locales, mais l'organisation offensive, l'organisation des razzias, comprenait au niveau de la province - naguère de la province entière, jadis des deux régiments de la province - les effectifs de la classe d'âge des guerriers assistés du plus jeune groupe de la classe des anciens. Chez les Kipsigis, l'organisation offensive militaire d'une province se basait sur la combinaison du système de classes d'âge et du système de régiments. Nous ne savons pas si l'organisation défensive des Nandi différait de leur organisation offensive. Dans une province, l'organisation offensive reposait sur les effectifs de la classe des guerriers. Comme l'explication en sera donnée plus loin, nous ignorons si l'organisation offensive des Nandi se fondait sur une combinaison du système de classes d'âge et du système de régiments, ou sur une combinaison du système de classes d'âge et du système territorial, ou sur une combinaison des trois. Quant à l'organisation offensive des Suk et des Keyu, encore moins connue, elle s'appuyait certainement sur le système de classes d'âge.

Autant que l'on puisse en juger d'après la littérature, le même système de clans totémiques existait chez les Nandi, les Kipsigis, les Suk et les Keyu. Les clans nandi (oret, pl. ortinuek) et kipsigis (oret, pl. ortinwek) ne pratiquaient généralement pas l'exogamie, et une assertion de Massam nous conduit à penser qu'il en était de même chez les Keyu.

Barton et Dundas affirment que les clans suk (or, pl. orten) étaient exogames, mais la confusion de leurs récits nous montre leur incompréhension de la nature des groupes qu'ils décrivent. Les clans nandi, kipsigis et suk se subdivisaient en de nombreuses branches souvent dénommées «familles » ou «sous-clans ». Chez les Kipsigis, lorsque ces branches ne se subdivisaient pas davantage, elles pratiquaient l'exogamie. Si elles se subdivisaient, les plus petits groupes pratiquaient l'exogamie, mais non les branches plus importantes. Les Nandi semblent avoir appliqué une règle similaire. Si nous ignorons l'attitude exacte des Suk et des Keyu à ce sujet, il est probable que leurs habitudes s'apparentaient à celles des deux autres peuples. Barton cite nombre de subdivisions claniques chez les Suk. Massam ne fait pas allusion à de semblables divisions chez les Keyu, mais son récit laisse supposer leur existence.

Peristiany pour les Kipsigis, Huntingford pour les Nandi, Massam pour les Keyu, Barton et Dundas pour les Suk, font état de la dispersion des clans ou branches de clan. Aucune relation ne semble avoir existé entre les clans ou les sous-clans de ces quatre peuples et les divisions territoriales.

Chez les Kipsigis, ni le clan ni le sous-clan ne représentaient un lignage, c'est-à-dire un groupe de gens liés par la généalogie. Le même phénomène se retrouve chez les Nandi et probablement chez les Suk et les Keyu. Chez les Nandi et les Kipsigis, les fonctions judiciaires appartenaient à la branche du clan et non au clan lui-même. La branche d'un clan kipsigis (kot-ap-chi) se devait de venger l'homicide d'un de ses membres et d'assister ceux-ci en les indemnisant pour les homicides et les autres torts. Selon HOLLIS, chez les Nandi, le clan assumait ces fonctions ; mais, en réalité, il se réfère à une branche du clan qu'il appelle «famille». Les écrits sur les Suk et les Keyu ne nous permettent pas de semblables affirmations quant aux fonctions de la branche du clan. Chez les Kipsigis et les Nandi, un homme qui tuait un membre du clan était rituellement souillé, mais il ne pouvait s'ensuivre ni vengeance, ni indemnisation. Rien ne nous permet de dire si telle était aussi la règle chez les Suk et les Keyu. 
Les clans n'assumaient de rôles politiques dans aucun de ces quatre peuples. L'organisation territoriale, l'organisation des régiments et l'organisation des classes d'âge ne dépendaient pas des structures claniques. Dans les relations politiques, seule la plus petite portion d'un clan avait une certaine importance, en cas de poursuites judiciaires et de règlements de querelles, et ses fonctions étaient toujours subordonnées à l'organisation locale. L'autorité n'était nulle part fondée sur le système clanique mais toujours sur les systèmes territorial, de régiments et de classes d'âge.

Autant qu'on puisse l'affirmer, les Nandi, les Keyu et les Suk, ainsi que probablement les autres tribus du groupe de langue nandi du Kenya, possédaient des organisations sociales comparables dans leurs grandes lignes à celles des Kipsigis. Cependant, d'après nos sources, les Kipsigis différaient des autres peuples par le rôle que jouait leur organisation en régiments dans la structure sociale. Barton affirme nettement « qu'à la différence des Nandi, les Kipsigis ne se répartissaient pas en secteurs géographiques à base militaire » ${ }^{1}$.

Selon Peristiany, on rencontrait, chez les Kipsigis, deux types d'organisation militaire. L'organisation défensive reposait sur le système territorial. Chaque village possédait sa troupe de guerriers sous l'autorité d'un chef dépendant lui-même du chef de district qui commandait toutes les troupes des villages. L'organisation offensive des razzias avait à la fois une base plus large et un principe différent. Elle se constituait de quatre régiments (puriet, pl. puriosiek) dont les membres provenaient de tous les coins du pays. Chaque membre appartenait au régiment de son père. Ces régiments n'agissaient pas en coordination ; seuls les membres d'une même province se battaient ensemble. Les sections provinciales des régiments se groupaient deux par deux, et chaque groupe menait ses razzias indépendamment de l'autre. Après que ce système eut conduit à une sévère défaite infligée par les Kisii, on en adopta un autre. Les forces offensives d'une province furent alors divisées en quatre compagnies qui reçurent chacune un nombre égal de guerriers provenant des quatre régiments. L'organisation antérieure en régiments ne se maintint que pour les cérémonies. Cette nouvelle organisation semble indiquer une tendance à rompre avec l'ancien système de régiments en tant qu'organisation militaire, et à le remplacer par une organisation militaire répondant mieux aux modifications de territoires. Cependant, les chefs de l'organisation militaire offensive étaient choisis sur la base du système de régiments et non sur la base du système territorial comme dans le cas de l'organisation défensive. Il en allait de même en ce qui concernait le recrutement des combattants d'une province qui n'appartenaient plus qu'à une seule organisation militaire. Les participants aux razzias faisaient partie de la classe des guerriers.

Bien que les régiments kipsigis aient été organisés en unités militaires et rituelles à l'intérieur d'une seule province, en tant que parties d'un système social ils constituaient des groupes qui dépassaient les limites des provinces et établissaient des liens entre des personnes séparées par des divisions territoriales. Un homme d'un puriet qui quittait sa province pour aller vivre dans une autre, appartenait jusqu'à sa mort au puriet où il était ne. Ses fils en étaient également membres. Chez les Nandi, au contraire, l'organisation en régiments se calquait sur l'organisation territoriale, et un régiment se composait des hommes d'une province. Le pororiet désignait à la fois la province et les hommes d'une province.

Juxon BARTON, Notes on the Kipsigis or Lumbwa Tribe of Kenya Colony, J.R.A.I., 1923, p. 46. 
Selon Huntingford, lorsqu'un homme quittait son pororiet pour s'établir dans un autre, après trois ans de résidence, il devenait membre de ce second pororiet. L'organisation militaire des Nandi n'apparaît donc pas comme structurellement distincte de l'organisation territoriale. C'est au moins la conclusion à laquelle je suis parvenu à partir de récits quelque peu contradictoires.

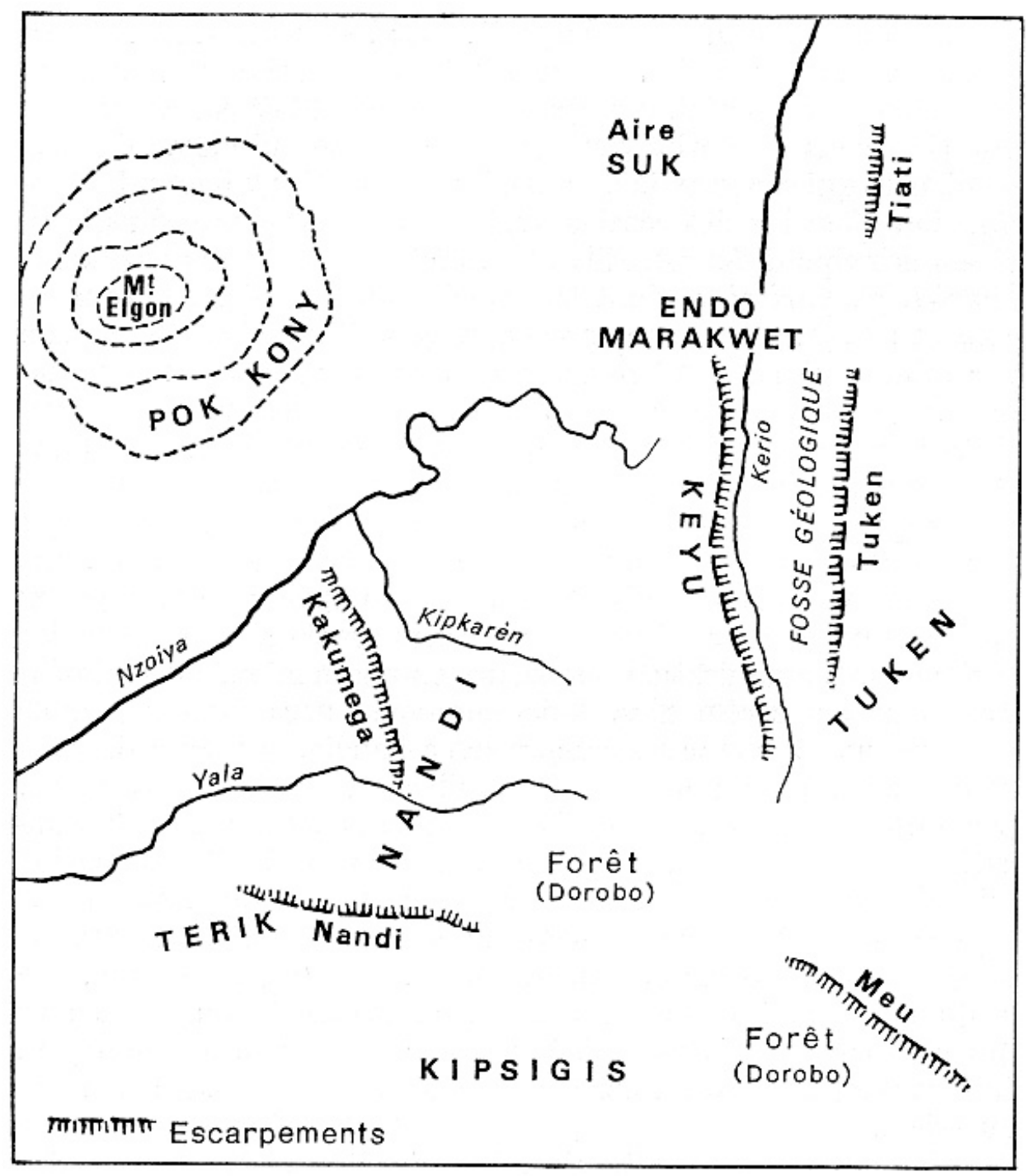

Zones occupées par les peuples de langue nandi du Kenya 
D'après HOLLIS et Huntingford, le pays nandi se divisait en six ${ }^{1}$ régions géographiques (emet, pl. emotinuek) sans signification politique, à la différence de l'unité territoriale ou province kipsigis (emet). L'unité politique nandi correspondant à la province kipsigis était le pororiet. Cet énoncé n'aurait entraîné aucune confusion, si le pororiet n'avait pas été décrit par certains, sans explication supplémentaire, comme étant un régiment. Dans son vocabulaire, HOLLIS cite ce mot comme équivalent d' « une compagnie de guerriers », alors qu'Huntingford oppose le système de régiments et le système territorial. Il précise que le pororiet est une division militaire qui a une signification territoriale.

Les récits de HOLLIS, Hemsted et Huntingford démontrent clairement que les pororosiek nandi étaient des groupes territoriaux à fonctions politiques. Tandis que les puriosiek kipsigis ne dépendaient d'aucune circonscription locale particulière mais se rencontraient dans toutes, les pororosiek nandi, à une ou deux exceptions près ${ }^{2}$, représentaient des unités territoriales bien définies. La nature de l'organisation militaire, dans les récits, demeure assez obscure. On ne peut établir avec certitude si l'organisation territoriale et l'organisation en régiments formaient un tout ou si elles différaient en dépit de leur coïncidence spatiale. Ainsi, tandis qu'Hollis décrit le siritiet comme une paroisse ou division territoriale d'une province (pororiet), Huntingford conteste cette affirmation et en fait une section de régiment. Hemsted la désigne comme étant une compagnie de district appartenant à un régiment. Il identifie ainsi l'organisation du régiment et l'organisation territoriale en ce qui concerne les activités militaires.

Hemsted est le seul à donner des détails sur l'organisation de l'armée. Chaque division, dit-il, avait son conseil, le «Conseil du clan» (kirwagel-ap-b oriel). On ne sait exactement s'il se réfère à une province (pororiet) ou à un district de province. Ce conseil, avant tout conseil de guerre, se composait des capitaines des compagnies de guerriers des différentes classes d'âge (kirwagik) sous la présidence du kirwagindet-ap-boriet. Il ajoute que les guerriers groupés en régiments, chacun sous l'autorité d'un capitaine du clan (kiplayat-apboriet), se divisaient en compagnies de districts (siritaiik) dirigées par un chef, le kiptayat. Ce récit reste très confus. La traduction de boriet par clan est presque certainement erronée, ce terme correspond bien entendu au régiment (puriet) kipsigis. En fait, la description de Hemsted des conseils de chefs de compagnies de guerriers des différentes classes d'âge concorde exactement avec la description faite par Peristiany du conseil kipsigis qui se réunissait pour choisir les nouveaux chefs de régiments à l'entrée des plus âgés dans la classe des anciens.

Malheureusement, Hemsted, lorsqu'il dresse la liste des provinces nandi, ne cite pas le terme vernaculaire. Certains les appellent pororosiek (sing. pororiet) et on se demande alors si Hemsted distinguait entre la province et le régiment quand il employait boriet pour désigner ce dernier. Huntingford estime que les Européens emploient parfois à tort, au lieu du terme pororiet, celui de poriet, lequel a en réalité le sens de «bataille». Cependant, Peristiany fait autorité pour la désignation du régiment kipsigis par le mot puriet.

\footnotetext{
Comme ces auteurs semblent avoir inclus dans leur liste deux des provinces kipsigis, il apparaîtrait que les Nandi, comme les Kipsigis, aient été divisés en quatre régions géographiques principales.

2 Selon Huntingford, quatre d'entre eux se scindèrent et une partie de chacun de ces pororosiek se dirigea vers le nord où elle s'installa.
} 
L'ignorance, chez les experts, de la signification des termes ethnologiques est à l'origine d'une grande part de la confusion, accentuée par l'absence de définition claire des mots employés. Ainsi, hormis le cas de pororiet, puriet, boriet et oret (clan), l'expression kirwagindet présente une certaine ambiguïté. Hemsted semble utiliser ce mot pour désigner un ex-chef de régiment nandi, tandis qu'il emploie kiptagat pour un chef en activité. Peristiany, dans les deux cas, se sert de kiptagat et réserve l'appellation kiruogindet pour les juges locaux. Barton parlant aussi des Kipsigis (op. cil., p. 46) utilise le mot kirowokindet pour le chef en activité. Cependant, il ne s'agit que de divergences mineures. Apparemment, les régiments nandi se formaient sur la base des provinces, à la différence des régiments kipsigis.

Nous n'avons pratiquement aucun élément nous permettant d'affirmer l'existence, chez les Keyu et les Suk, d'une organisation semblable à celle des régiments kipsigis. Elle se rapprochait plutôt de celle des Nandi. Beech suppose que les anciens des provinces Suk élisaient deux hommes de la classe des guerriers pour commander les combattants.

Je conclurai cette brève analyse en dégageant quelques caractéristiques significatives des structures sociales des peuples de langue nandi.

1. - Le groupe des peuples de langue nandi appartient à un type de sociétés africaines décentralisées. On ne rencontre chez eux aucune société à l'autorité fortement centralisée et aux institutions gouvernementales développées. Chez ces peuples, le système politique se composait d'un certain nombre de segments locaux qui se subdivisaient à leur tour. Ses institutions juridiques et militaires ne dépendaient pas d'une organisation bureaucratique dont l'autorité centrale aurait lancé ses mots d'ordre par le truchement de délégués administratifs et militaires. L'organisation politique était démocratique. Les membres d'une communauté locale réglaient leurs propres affaires, et ils étaient équitablement représentés aux conseils qui statuaient sur les problèmes des communautés locales plus vastes auxquelles ils appartenaient. Bien que la loi s'étendît à toute la région qu'habitait un peuple, les organisations judiciaire, militaire et rituelle étaient propres à chaque section territoriale. Lorsque les habitants d'une province tout entière étaient concernés, l'organisation était provinciale, lorsque seuls les habitants d'une unité territoriale réduite étaient en cause, l'organisation se limitait à cette unité : le district, le village ou même le hameau. Au lieu d'une délégation d'autorité issue d'un pouvoir central, il y avait délégation d'autorité des petites unités locales vers celles de plus grande importance. Les groupes territoriaux organisés les plus vastes étaient les provinces, l'emet kipsigis, le pororiet nandi. Cependant, toutes les provinces appartenaient à un même système politique, et des groupes sociaux d'origine non territoriale chevauchaient les segments locaux. Les différentes provinces possédaient des intérêts semblables plutôt que communs. La position indéterminée des prophètes dans la structure politique en est le reflet.

2. - Le système décentralisé des peuples nandi était basé sur un principe structurel différent du type nilotique, dans lequel la structure lignagère et la structure territoriale allaient presque toujours de pair. J'ai décrit une société de ce type, celle des Nuer du Soudan. Les Luo du Kenya en sont un autre exemple. Les groupes territoriaux recoupaient les groupes lignagers de façon telle qu'on pouvait toujours se référer au système territorial en terme de 
structure lignagère, et lorsqu'il existait une organisation, elle reposait sur une base lignagère. Les segments locaux s'identifiaient structurellement avec les segments lignagers dans les relations politiques. On peut dire que chez ces peuples nilotiques, les clans avaient des fonctions politiques. Une corrélation existait peut-être entre la fonction politique du clan et sa structure lignagère.

Les clans kipsigis, nandi, suk et keyu, dont nous parlons, pas plus qu'aucun de leurs segments, ne recoupaient les communautés locales. Leur structure ne sous-tendait ni le système territorial, ni l'organisation territoriale. Aussi n'avaient-ils pas une structure lignagère, mais une forme différente de segmentation. Le terme «sous-clan », d'usage fréquent en anthropologie, peut s'employer pour désigner un segment clanique dont la structure ne repose pas sur la généalogie. Il existe peut-être une corrélation entre l'absence de fonction politique et l'absence de structure généalogique dans le système clanique.

3. - Parmi ces peuples, la structure politique ne correspondait pas à la structure sousclanique. A la structure clanique se substituaient un certain nombre d'autres institutions dont le rôle politique mérite une plus large explication. Chez les Nandi, le système de régiments, en correspondance avec le système territorial, semble avoir été, d'une part, le principe structurel de ce système, et d'autre part, avec le système de classes d'âge, la base de l'organisation de segments locaux plus importants, au moins pour certaines activités. Chez les Kipsigis, le système territorial ne correspondait à aucun autre système social local, mais, là encore, le système de régiments et le système de classes d'âge étaient à la base de l'organisation de certaines activités dans les segments locaux plus importants. Pour d'autres activités, l'organisation des segments locaux se fondait presque entièrement sur un principe territorial. Pour certaines raisons, il se peut que l'organisation locale nandi ait été presque entièrement basée sur le principe territorial, mais les renseignements dont nous disposons ne nous permettent pas de porter un jugement définitif dans ce sens. La pauvreté de l'information empêche une analyse structurelle claire des systèmes des Keyu et des Suk. Nous pouvons cependant conclure, d'après les minces preuves que nous possédons, que par les traits principaux de leurs organisations et systèmes sociaux ils se rapprochaient des Nandi.

4. - Parmi tous les peuples de langue nandi, les classes d'âge apparaissent comme l'un des traits structurels dominants. Les classes s'étendaient au-delà des frontières provinciales et même d'un peuple à l'autre. Chez les Kipsigis, l'organisation des razzias à l'intérieur d'une province se fondait sur la combinaison du système de classes d'âge et du système de régiments ; chez des Nandi, sur la combinaison du système de classes d'âge et du système de régiments ou du système territorial ou sur les trois à la fois. Chez les Suk et les Keyu, le système de classes d'âge avait certainement des fonctions militaires, bien que l'on ignore l'organisation précise de leurs razzias.

5. - Dans une classification structurelle des peuples est-africains, les traits significatifs de ces quatre sociétés sont : un système territorial de provinces comprenant une organisation politique basée sur le principe territorial dans le domaine judiciaire, et, en certains cas, dans le domaine de la défense militaire l'absence d'autorité centrale et d'institutions 
gouvernementales des opérations collectives de procédure criminelle; la non-importance politique du système clanique; et la présence d'un système de classes d'âge à fonctions politiques, notamment dans l'organisation militaire offensive. Le système de régiments peut distinguer les Kipsigis des autres peuples, mais des recherches approfondies les montreraient peut-être, à cet égard, moins différents que les études ne le suggèrent. 


\section{Chapitre 4 \\ "Quelques expressions collectives de l'obscénité en Afrique" * (1920)}

\section{I - Introduction}

$\underline{\text { Retour à la table des matières }}$

Pour quiconque vit parmi des peuples primitifs, il n'est pas rare de rencontrer des expressions verbales et comportementales de «l'obscénité ». Fréquemment cette « obscénité » n'est ni proférée par un individu sous le coup de la colère, ni condamnée au nom du mauvais goût, mais collective, autorisée et même prescrite par la société. Certains Européens s'excusent de nous présenter une telle indécence, mais en même temps, ils nous en fournissent un récit complet et impartial; quelques-uns, comme Junod, Smith et Dale essaient même de nous expliquer ces « indécences ».

Cet article, qui parut pour la première fois en 1929 dans le J.R.A.I., faisait partie d'une thèse de doctorat en philosophie présentée à l'Université de Londres, sur «The Social Organization of the Azande of the Bahrel-Ghazal Province of the Anglo-Egyptian Sudan ». Les matériaux sur le Soudan avaient été rassemblés en 1926-1927, lors d'une expédition pour le compte du gouvernement du Soudan anglo-égyptien. Une aide financière supplémentaire fut accordée par la Royal Society et les Laura Spelman Rockfeller Trustees. 
Cependant, la plupart des Européens, bien qu'incapables de découvrir les obscénités de leur propre société, les perçoivent parmi les indigènes parce qu'elles leur sont étrangères, et les condamnent sans appel. Il semble que le sens moral du Blanc, offensé par ces obscénités, rende compte de telles condamnations. Inutile d'ajouter qu'aucun de ces critiques ne tente une quelconque explication. En conséquence, ces obscénités sont stigmatisées comme antisociales et souvent condamnées par la loi. On les décrit comme «au-delà de la bestialité » ou « trop horribles pour être répétées ».

Dans cet article, je me propose de rassembler les récits les mieux documentés sur les obscénités en Afrique, afin que les données azande, objets de mon étude, ne constituent pas des exemples isolés, mais puissent être comparées à tout un ensemble de faits sociaux analogues.

\section{II - L’obscénité chez la Ba-Ila}

\section{$\underline{\text { Retour à la table des matières }}$}

Chez les Ba-Ila de la Rhodésie du Nord ${ }^{1}$, les chants licencieux sont non seulement autorisés, mais essentiels au rituel en certaines circonstances : les semailles, la pêche lwando, la mise à l'eau d'une nouvelle pirogue, la fonte du fer, l'initiation, les funérailles et les makubi. Ils accompagnent également la danse kashimbo (II, p. 191).

Lors des semailles, de la fonte du fer, de la pêche, de la mise

l'eau des pirogues, les chants érotiques sont liés à une activité économique ; dans les autres cas, à un cérémonial religieux.

Mais bien que Smith et Dale nous citent les circonstances économiques à l'origine de ces chants érotiques, leurs récits manquent souvent de détails. A propos des semailles, on sait que la femme, aidée des membres de sa maison, "commence à houer le sol et à entasser l'herbe et les détritus jusqu'à ce qu'ils soient assez secs pour les brûler, tandis que, pendant ce temps, son mari houe son propre petit lopin. Aussitôt le sol préparé, on procède aux semailles » (1, p. 137). Il semble que ce soit durant ce travail collectif de préparation du sol et d'enfouissement des graines par toute la maisonnée, que les femmes chantent ces chants licencieux,

Lors de la pêche lwando, les hommes quittent le village et campent au bord d'une rivière. Une grande nasse de roseaux lâchement tressés sert d'épuisette ; les hommes s'avancent dans la rivière en poussant cette nasse devant eux, et y enferment les poissons qu'ils sortent ensuite

\footnotetext{
${ }^{1}$ Toutes les références sur les Ba-Ila proviennent du Révérend Edwin W. SMITH et du capitaine A. M. DALE, The Ila-speaking Peoples of Northern Rhodesia, 1920.
} 
de l'eau. Nous voici à nouveau en présence d'une activité collective d'envergure, mais nous ignorons comment les chants s'intègrent dans cette opération (1, pp. 161-162, 168-169).

D'après les descriptions de fabrication des pirogues et d'après une photo de l'une d'elles achevée, il est évident que le transport à la rivière représente un énorme travail qui nécessite un grand nombre de participants. Nous aimerions, là encore, des détails supplémentaires sur la manière dont les chants accompagnent cette activité (1, pp. 200-202).

Chez les Ba-Ila, la fonte du fer est un travail long et important que règlent de sévères tabous. Il faut couper des arbres pour en faire du charbon de bois, extraire le minerai de fer, puiser de l'eau, amasser de l'argile et construire les fours. Il semble que tous les hommes des villages participent à ces opérations effectuées loin des habitations et qu'ils vivent dans des abris spécialement dressés pour la circonstance. Quant aux chants érotiques, nous savons seulement qu'ils accompagnent les travaux.

«Lorsque notre informateur raconte que le langage grossier n'est pas prohibé, il pense particulièrement aux chants, la plupart du temps licencieux, exécutés pendant les opérations » (I, p. 208). Les auteurs donnent quelques exemples de ces chants, Shampala yamwandauka ( "L'homme avec son gland nu tout éclaté se ressent du printemps qui le fait éjaculer») (I, pp. 207-208).

Les cérémonies religieuses de l'initiation, des funérailles et les fêtes en l'honneur des « divinités » autorisent la licence.

«A ces occasions, notamment celles du mwandu et du chisungu, liées à l'initiation des filles, les danses et les chants sont particulièrement obscènes. Peut-être possèdent-ils quelque sens occulte - nous l'avons noté en ce qui concerne les chants - mais leur raison apparente est d'exciter au maximum les passions. » On nous dit aussi que, lors de l'initiation des filles, « la danse du chisungu dure pendant deux ou trois jours et deux ou trois nuits et qu'elle occasionne une débauche sans frein » (II, pp. 18-26). On y danse également le kashimbo.

Pendant les fêtes funéraires, les femmes endeuillées chantent des chants érotiques dont Smith et Dale donnent plusieurs exemples :

«Ma!ma_! ma_! diakomena itoni diakwe !

ndia mulolobozho

hudikwete kudilolobola. »

(«Mon Dieu 1 Mon Dieu ! Que son pénis est long 1 Il n'en finit pas 1 Il a dû joliment servir. »)

Les auteurs continuent : «Lorsque nous avons exprimé notre étonnement d'entendre les femmes chanter ainsi - car ce sont les femmes qui chantent -, les anciens nous ont répondu par un proverbe : Ushildilwe taitwa ku bushu «( On ne passe pas devant le visage d'une personne en deuil »), c'est-à-dire il (ou elle) a toute licence pour faire ce qui lui plait. Dans les circonstances habituelles, il serait tabou pour les femmes de proférer de telles paroles devant les hommes ; mais, aux funérailles, toute contrainte disparaît. Chacun fait ce qui lui plaît. On peut enlever la paille des toits de chaume, piller les champs de céréales; toutes les 
passions se déchaînent et personne ne peut se plaindre de dommages, de vol, d'adultère » (II, p. 113).

Parmi les Ba-Ila, une fête annuelle au moins, appelée ikubi (pl. makubi), est célébrée en l'honneur de chaque «demi-dieu » ou esprit ancestral de marque. Les auteurs résument ainsi ce qui s'y passe : "Les makubi se déroulent toujours avec la même uniformité. Bière, danses, chants licencieux abondent. Les hommes chargent de tous côtés avec leurs lances. La débauche règne. A bien des égards, on peut comparer cette fête annuelle aux Saturnales » (II, p. 191).

Avant d'aborder les Thonga, je résumerai ce qui touche aux Ba-Ila :

1) La société ne tolère pas habituellement les chants licencieux ;

2) Elle les autorise : Soit lors d'une cérémonie religieuse importante ;

3) Soit lors d'activités collectives d'ordre économique, particulièrement longues et pénibles.

\section{III - L'obscénité chez les Ba-Thonga}

\section{Retour à la table des matières}

Nous observons que chez les Ba-Thonga du Transvaal ${ }^{1}$, les chants et les comportements érotiques sont autorisés à peu près dans les mêmes circonstances que chez les Ba-Ila, mais il est souvent plus difficile de différencier les occasions d'ordre économique des occasions d'ordre religieux, car chants et danses accompagnent parfois un travail effectué en relation avec une cérémonie.

On apprend que dans le sungi, ou camp de la circoncision, les tabous sexuels les plus sévères doivent être respectés par tous les occupants, et que les gens du village ne doivent ni faire de bruit, ni se quereller. "Chose étrange, pendant ce temps, le langage obscène est permis, recommandé même - c'est là un contraste que nous trouverons souvent pendant les périodes de marge. Quelques-unes des formules contiennent des expressions tabous en temps ordinaire. Lorsque les femmes apportent la nourriture à quelque distance de l'entrée du sungi, les bergers qui vont la prendre peuvent leur adresser toutes les paroles impures qu'ils veulent. Les mères ont elles-mêmes le droit de chanter des chansons obscènes lorsqu'elles pilent le maïs pour le sungi » $(1$, p. 80$)$.

«La coutume veut que les femmes, les mères des circoncis, apportent une grande quantité de farine de maïs bouillie, au moins le double de ce qui est nécessaire aux garçons.

\footnotetext{
Toutes les références sur les Ba-Thonga sont puisées dans Henri A. JUNOD, Mœurs et coutumes des Bantous; la vie d'une tribu africaine, Payot, 1936.
} 
Si l'une d'elles venait à y manquer, elle serait punie à un moment donné. Elles déposent leurs marmites à une certaine distance, assez loin pour qu'elles ne puissent rien voir du ngoma, et elles crient : «Ha tsoo! Nous brûlons !» Ce qui veut dire : «Nos têtes sont «endolories d'avoir porté si loin les marmites. » Les bergers accourent à leur rencontre et leur répondent par des plaisanteries d'un goût douteux : « Nous savons bien ce qui vous « brûle, etc. N'est-ce pas la règle du ngoma ?»» $(\mathrm{I}, \mathrm{p} .82)$.

Les formules secrètes des initiés sont particulièrement obscènes. Lorsqu'on veut savoir si un garçon a été circoncis, on lui dit «La bête qui doit être ouverte en commençant par derrière » s'il a été initié, il répond : « le crocodile », puis ajoute : « le couteau qui circoncit» $(1$, p. 96). La formule fait allusion à la manière dont le crocodile mâle recouvre sa femelle et à celle dont un garçon viole la jeune fille vierge afin de tracer la voie aux adultes qui suivront. L'auteur, estimant beaucoup de ces formules trop triviales, les traduit en latin.

Nous remarquons donc que les chants et expressions obscènes accompagnent les rites importants de la circoncision, de même que le pilage particulièrement laborieux d'une grande quantité de maïs pour les circoncis.

Dans les clans du Nord, lorsque les filles atteignent l'âge nubile, on les isole. "Puis commence une période de marge qui dure un mois. Trois ou quatre jeunes filles sont initiées ensemble... Chaque matin, on les conduit à l'étang et on les plonge dans l'eau jusqu'au cou. D'autres jeunes filles initiées ou des femmes les accompagnent en chantant des chansons obscènes et chassent à coups de bâton tout homme qui se trouve sur le chemin, car aucun homme ne doit voir la jeune fille pendant cette période. Si un homme approchait par hasard du groupe, les femmes lui poseraient les questions contenues dans les formules secrètes de l'école de la circoncision, non pas les longues et les compliquées, mais celles qui sont courtes, et probablement celles qui renferment les mots obscènes... Lorsque le cortège des femmes qui accompagne les initiées est rentré, ces dernières sont enfermées dans la hutte. Leurs mères adoptives ou d'autres femmes les tourmentent, les pincent, les égratignent ; il faut qu'elles écoutent également les chansons obscènes qu'on leur chante » (1, p. 169).

Deux ou trois mois après la mort d'un Ba-Thonga a lieu une cérémonie à laquelle assistent tous les membres de la famille du défunt et au cours de laquelle on détruit la case mortuaire. Une partie du rituel consiste à sacrifier un chevreau et quelques volailles. "Alors, pendant que les batukula et les vieillards étaient occupés auprès des victimes, les découpant et pressant le psany (herbe à demi digérée) hors des entrailles, les autres assistants se mirent à chanter et à danser. D'abord une femme âgée, au teint très clair et à la face méphistophélique, très grande, avec un sourire étrangement licencieux, vint au milieu de la place, ouvrit tout grands ses bras et suma, se mit à chanter. En même temps qu'elle chantait, elle accomplissait avec ses cuisses une étrange mimique. Cette mimique prit de plus en plus un caractère lascif ; elle devint une véritable danse du ventre, tellement immorale que les hommes baissèrent les yeux comme s'ils avaient craint qu'elle n'enlevât tous ses vêtements... Les paroles de ses chants étaient également d'un caractère équivoque. Elle décrivait une femme adultère allant, pendant la nuit, d'une hutte à l'autre chercher des amants et frappant aux parois... Tout ceci nous semble très immoral. N'oublions pas cependant que, dans l'esprit des Thonga, ces chants qui sont tabous dans la vie ordinaire, sont tout spécialement appropriés aux périodes de deuil. «Ces femmes ont été découvertes par la mort de leur mari, me disait Mboza, rien ne les retient plus. Elles sont pleines d'amertume lorsqu'elles exécutent ces danses lascives. » La 
raison en est peut-être plus profonde car ce ne sont pas seulement les veuves qui chantent ces paroles. Nous sommes encore dans une période de marge, la période du deuil, et ces phaseslà de la vie sont marquées pour les Bantous par cet étrange contraste : prohibition des rapports sexuels en même temps qu'un flot éhonté de paroles et de gesticulations obscènes » (1, pp. 152-154).

Chez tous les peuples africains, la construction d'une nouvelle habitation constitue peutêtre la tâche à caractère économique la plus importante, aussi s'accompagne-t-elle souvent de rites religieux. Parmi les clans du nord du pays des Thonga existe une période spéciale de transfert d'un village à un autre qui dure environ un mois, pendant lequel les tabous les plus rigoureux doivent être respectés.

«Lorsque tous les murs sont prêts, les toits sont portés de l'ancien au nouveau village par tous les hommes ensemble. Ils soulèvent chaque toit sur leurs épaules après avoir enlevé le chaume qui les recouvre et sortent du village, non par l'entrée principale, mais par l'une des portes de derrière, élargie dans ce but. Une large route a été préparée à travers la brousse. Ils la suivent en marchant aussi vite qu'ils le peuvent et en chantant les chants obscènes qui sont réservés à ces occasions spéciales. Dans ces chants, ils insultent les femmes qui les accompagnent en portant les paniers, les mortiers, les pilons. "Le village est brisé, il en est de même pour les lois ordinaires de la vie. Les insultes qui sont tabou sont maintenant permises » (Mboza). Cette suspension de la moralité dans le langage n'est autorisée que le jour où les toits sont portés au nouveau village. Quelques jours plus tard, les femmes prennent leur revanche lorsqu'elles enduisent d'argile le sol des huttes ; elles aussi chantent leurs chants et insultent les hommes. Mais tout cela n'est qu'une plaisanterie. C'est un grand jour de réjouissance pour les tinamu qui se taquinent mutuellement autant qu'il leur plaît. Pendant cette période un homme peut manquer de respect même à sa grande mukonwana » (1, p. 306). Chez les Ba-Thonga, ces chants ne sont pas autorisés lors du transfert d'une seule case.

Bien que la construction d'un nouveau village débute et s'achève par des cérémonies, cette activité est plutôt d'ordre économique que rituel. On n'exécute des chants obscènes que dans les deux cas suivants d'activité collective : lors du trainsport fatigant et déplaisant des toits de cases et lors du bousillage du sol.

Lors d'une période de sécheresse prolongée, les Ba-Thonga offrent un sacrifice. «Puis les femmes se rassemblent. Elles doivent ôter tous leurs vêtements et s'entourer seulement les reins avec des herbes, et, marchant à petits sauts, chantant un chant spécial Mpfula, nana! ( "Pluie, tombe »), elles se rendent à tous les endroits où des enfants nés prématurément ont été enterrés dans le sable des collines, elles exhument ce qui reste de ces petits corps dans des tessons, et réunissent toutes ces matières impures en un lieu secret, de telle sorte que les enfants ne puissent pas voir ce qu'elles font. En outre, elles versent de l'eau sur ces tombeaux afin de les «éteindre » (timula). Le soir du même jour, elles vont enterrer ces impuretés dans la boue, près de la rivière. Aucun être masculin ne doit s'approcher durant l'opération ; les femmes auraient le droit de frapper l'imprudent et de lui poser les questions obscènes des formules de la circoncision; l'homme leur répondrait alors en employant les paroles les plus impures qu'il saurait trouver, car tous les tabous du langage sont supprimés ce jour-là ; la nudité elle-même n'est plus défendue, car, disait Viguet, "c'est la loi du pays ». Chacun consent à la suppression des règles ordinaires de la vie » (II, p. 273). 
La cérémonie pour faire tomber la pluie se caractérise donc par l'obscénité dans le langage et par la nudité, qui sont strictement interdites en temps normal.

La dernière cérémonie Thonga décrite est celle du nunu. Le nunu est un insecte qui provoque de grands ravages parmi les haricots et le maïs. Les chefs envoient les femmes ramasser cet insecte sur les tiges de haricots et une jeune fille, une jumelle, va les jeter dans le lac voisin. «Derrière elle, marche toute la troupe des femmes, les bras, la taille et la tête couverts d'herbe, portant des branches de manioc aux grandes feuilles qu'elles agitent de-ci de-là, en chantant... » (II, p. 394).

Lorsque la jumelle a jeté les insectes dans le lac, « les hurlements sauvages commencent à s'élever toujours plus forts, et les femmes chantent leurs chants obscènes (la ku ruketela), qu'elles n'oseraient jamais chanter en temps ordinaire, et qui sont réserves pour ces cérémonies, pour la recherche de la pluie et pour la chasse du nunu » $(11, p .395)$.

En analysant les descriptions de ces attitudes licencieuses, nous observons que :

1) Comme chez les Ba-Ila, ces formes spécifiques d'obscénité dans les chants, les paroles et les actes, ne sont pas habituellement autorisées par la société.

2) Lorsqu'elles sont permises, c'est au cours de cérémonies importantes liées aux rites de la circoncision des garçons, de l'initiation des filles, des fêtes funéraires, des cérémonies de la pluie et du nunu.

3) $\mathrm{Ou}$ bien en relation avec quelque activité économique collective pénible et longue : le transport des toits, le bousillage des sols lors de la construction d'un nouveau village, ou encore le pilage du maïs, par une femme (ou plusieurs), pour le camp de la circoncision.

\section{IV - L'obscénité chez les Akamba}

\section{$\underline{\text { Retour à la table des matières }}$}

Je ne veux pas surcharger cet article d'exemples, mais il convient de mentionner, quant à notre sujet, les apports de l'excellent traité de Lindblom sur les Akamba du Kenya d'Afrique orientale ${ }^{1}$.

Chez les Akamba, filles et garçons vont ensemble au camp d'initiation où ils chantent des chansons liées aux rites de la circoncision. "Certaines, exécutées en alternance par les garçons et les filles, sont extrêmement obscènes. En voici un exemple

\footnotetext{
1 Toutes les références sur les Akamba sont puisées dans The Akamba in British East Africa, 1920.
} 
« Les garçons entonnent :

«Hae, hae, écoute!

Le kino (sexe féminin) est idiot,

Il se tient sous les vêtements.

Hae, hae !»

« Et les filles répondent :

«Sais-tu, eeh, écoute !

Le kea (sexe masculin) est idiot.

Il se tient entre les testicules;

Il est idiot,

Il laisse le kino s'engraisser à ses dépens »

ce qui signifie : les rapports sexuels amaigrissent l'homme et font grossir la femme. Tout comme les chants, la conversation se révèle d'un goût fort douteux. D'après mon informateur, elle vise d'emblée à montrer qu'aucun sentiment de honte n'a place en ces circonstances, alors que, dans la vie quotidienne, une grande pudeur entoure ces sujets » (p. 50).

Dans les actes, l'obscénité est de règle comme dans les chants. Chacun des initiés, au cours des secondes cérémonies de la circoncision, doit maintenir son pénis jusqu'à érection. Alors, on attache à la verge un morceau de bois, et l'initié se promène ainsi au milieu des éclats de rire. Pendant ces mêmes cérémonies, on creuse un petit trou dans le sol, et les initiés doivent, chacun leur tour, éjaculer dans ce trou, symbole du vagin. Chaque initié doit aussi copuler avec un autre initié pour montrer comment il accomplit l'acte sexuel avec une femme. "Les chants sont d'une indescriptible licence. » Il y a débauche générale et les inities, armés de bâtons, terrorisent tout le pays (p. 65).

En période de sécheresse, les femmes akamba exécutent des rites semblables à ceux décrits par Junod chez les Ba-Thonga. Elles battent le tambour et marchent d'un village à l'autre, puis, en force, elles se rendent chez le sorcier.

En 1911, Lindblom rencontra le cortège des femmes et nota quelques-uns de leurs chants :

$\ll$ Ea, eeh!

Nous venons de loin

Chercher du sel pour le kino (sexe féminin).

Pénis dresse-toi.

Uh, uh !»

ce qu'il traduit librement par : «Nous voulons de la pluie pour avoir de quoi nourrir nos maris qui ne peuvent accomplir leurs devoirs sexuels si la faim les affaiblit. » 
Lindblom dit que les chants de la circoncision, d'âge immémorial, sont les mêmes dans tout l'Ukamba, tandis que les chants ordinaires sont locaux et éphémères. Il ne précise pas si les chants pour faire tomber la pluie se transmettent d'une génération à l'autre (p. 276).

Chez les Akamba, nous nous apercevons que

1) Certaines expressions collectives de l'obscénité sont habituellement prohibées par la société ;

2) Elles sont autorisées aux cérémonies de la circoncision et aux cérémonies pour faire tomber la pluie.

\section{V - L'obscénité chez les Azande}

$\underline{\text { Retour à la table des matières }}$

Je m'intéressai à ce problème de l'obscénité prescrite par la société, lorsque je me trouvai parmi les Azande de la région de Bahr-el-Ghazal au Soudan anglo-égyptien ${ }^{1}$. Les Azande ont quelques chants particulièrement obscènes qu'ils considèrent comme déplacés et choquants si on les chante en dehors d'un certain contexte social déterminé. Quelques années après la mort d'un homme ou d'une femme, ses parents offrent un festin à l'occasion duquel on place un tas de pierres sur la tombe du défunt. Ce festin nécessite des préparatifs considérables. Parmi ceux-ci, le plus gros travail consiste, pour les femmes, à battre une grande quantité de mil qu'on laissera germer avant de le moudre. Le mil sert à fabriquer la bière dont on boira quelques pots au festin, mais dont la plupart seront distribués, selon un système d'échange, par le maître du festin, à sa belle-famille.

Après une cérémonie religieuse en l'honneur de l'âme des défunts, on entasse le mil au centre de la concession, puis les femmes, les parentes, les voisines du maître du festin commencent à le battre avec de longs fléaux. Ce faisant, elles tournent autour du mil en chantant des chants obscènes, les seuls transmis de génération en génération qui soient connus dans le pays tout entier ; les autres sont régionaux et transitoires. On les chante sur un rythme rapide, en mesure avec les coups de fléaux qui frappent le grain en cadence et à intervalles réguliers. Généralement, une ou deux femmes dirigent l'opération. Elles se tiennent au milieu, mènent les chants et exécutent des mouvements obscènes. Voici l'un de ces chants ${ }^{2}$

« Na ta pumbo, wili mbia nenge ro yo.

Pour une étude complète sur les Azande, cf. LAGAE, Les Azande, 1926, et LARKEN, An Account of the Zande, Sudan Notes and Records, 1926, et Impressions of the Azande, ibid., 1927.

2 C'est la Major Larken qui me fit d'abord remarquer l'existence de ces chants dont je donne ici un ou deux exemples. 
«Maîtresse du festin, tu as une petite pierre dans ta vulve.

Ba semba li pumbo, wili mbia nenge ro yo. Maître du festin, tu as une petite pierre dans ta vulve.

Agume pumbo, wili mbia nenge ro yo.

Parents qui assistez au festin, vous avez une petite pierre dans votre [vulve.

Wili kawili pumbo, wili mbia nenge ro yo.

Fils de mon frère qui participe au festin, tu as une petite pierre dans [ta vulve.

A ta pumbo, wili mbia nenge ro yo. »

Vous tous qui êtes au festin, vous avez une petite pierre dans votre [vulve. »

Pendant ces chants, les hommes restent à l'écart de peur d'être insultés.

Environ un an après le battage du mil, le festin a lieu. Plusieurs jours auparavant, les femmes écrasent le grain germé pour en faire de la bière. De six à dix meules de pierre, et même plus, sont disposées en cercle à l'abri d'un grenier.

Tout d'abord, les anciens célèbrent une cérémonie en l'honneur de l'âme des morts, à laquelle s'ajoutent des rites magiques pour se protéger des sorciers et pour influencer les échanges rituels de biens lors du festin. Lorsqu'ils ont terminé, ils se mettent à chanter les plus connus de ces chants qui accompagnent le battage ou la mouture. Après quelques couplets, ils quittent les meules et les femmes viennent s'installer pour commencer à moudre. La farine tombe et s'entasse au centre.

Une ou deux femmes dirigent toujours les chants. Le travail ne cesse jamais, les femmes qui chantent vont remplacer celles qui sont fatiguées de moudre. La mouture se fait au rythme des chants : toutes les femmes poussent leurs meules en cadence.

Elles commencent la mouture par ce chant :

«Oo, oo, ngongo li ngoto, nenge oo ee ee !»

«Oo! Oo! A la verticale du clitoris, le vagin oo - ee - ee ! »

Cette phrase sert de refrain tout au long du chant qui comporte de nombreux couplets comme ceux-ci :

« Kira o, kira o, nenge na yemba ro o. »

«Pénis o ! Pénis o ! Le vagin t'appelle. »

ou :

« Agbio o, agbio, agbio ba kira yo zegio. »

«Les parents de la belle-famille o ! Les parents de la belle-famille 1 Les [parents de la belle-famille sortent leur pénis. » 
Je ne cite que quelques lignes de ce très long chant.

Les allusions aux parents sont strictement interdites en temps normal. Les chants s'accompagnent de gestes obscènes, et les hommes se gardent bien d'approcher les femmes pendant ce travail.

On m'a dit que lors de la construction du pont sur la rivière Sueh en mars 1927, les hommes exécutèrent certains de ces chants, durant ce travail long et fastidieux pour le compte du gouvernement.

Les expressions grossières et insultantes, proférées sous le coup de la colère, abondent chez les Azande comme chez nous. Mais elles ne sont certainement pas obligatoires et elles conduisent fréquemment au tumulte et à des poursuites judiciaires. D'autre part, lorsqu'un neveu pille les biens de son oncle maternel, faute contre laquelle aucun châtiment n'est prévu dans la loi indigène, le comportement des femmes de l'oncle semble voisin de ceux déjà décrits dans cet article. Les femmes déchirent leur cache-sexe de fibres et se précipitent toutes nues derrière l'intrus en lui criant des insultes grossières accompagnées de gestes impudiques. Nous mentionnons ces cas où, bien que l'obscénité soit permise, elle n'est ni obligatoire, ni liée à une réaction communautaire.

Les sociétés secrètes azande offrent aussi des exemples d'obscénité, mais on sait peu de chose de ces associations. Mes notes sont trop incomplètes pour fournir un récit détaillé de leurs cérémonies. Je ne voulais pas, lors de ma première visite, avoir l'air trop inquisiteur visà-vis de ces associations condamnées par les Blancs. L'indécence de ces cérémonies secrètes semble s'apparenter à celle des cérémonies d'initiation, comme on l'observe, pour les Wapare d'Afrique de l'Est, dans le Jahrbuch des Städtischen Museums für Völkerkunde zu Leipzig, 1913.

Résumons les données azande :

1) L'obscénité, normalement interdite par la société, est approuvée en certaines occasions ;

2) Elle est autorisée lors du battage et de la mouture collective du mil pour préparer les fêtes des défunts ;

3) Elle est aussi permise dans certaines coutumes ayant trait aux relations entre neveu et oncle maternel, et dans les cérémonies des sociétés secrètes.

Il existe probablement beaucoup d'autres exemples d'obscénité dans les sociétés africaines, mais une extrême prudence de jugement s'impose sur ce sujet, car les comptes rendus relèvent souvent de simples opinions ou impressions exprimées par un observateur inexpérimenté et partial. 


\section{VI - L'obscénité chez les Lango, les Didinga, les Lugbwara et les Ingassana}

\section{Retour à la table des matières}

Il m'est agréable de pouvoir compléter les données de cet essai par les observations d'un homme aussi impartial que Driberg. Dans son ouvrage sur les Lango d'Uganda, il décrit les cérémonies obscènes qui accompagnent la naissance des jumeaux, et il ajoute, dans une communication orale, que les chants de ces cérémonies se transmettent d'une génération à l'autre à la différence des autres chansons à l'existence passagère. Il pense qu'on les enseigne probablement au cours de l'initiation des filles. Lors des cérémonies d'initiation des garçons et des filles de cette tribu, on exécute des chants obscènes, de même que durant les cérémonies pour faire tomber la pluie. Dans ce dernier cas, un symbolisme sexuel obscurcit souvent la signification des chants, par exemple :

« Kwaich obolo ibe te oduru. »

«Le léopard se frotte la queue au figuier. »

(Le pénis se frotte aux parois du sexe féminin.)

Driberg nous précise aussi que l'initiation des hommes et des femmes chez les Didinga du Soudan anglo-égyptien est à l'origine d'une débauche générale accompagnée de chants licencieux. Chez ce même peuple, la construction des cairns, près des plantations, est, pour les femmes, l'occasion de chants érotiques. Elles jurent ensuite sur ces cairns de ne pas garder le produit des récoltes pour leur seul usage, mais d'en faire bénéficier le clan tout entier. Les jeunes filles chantent aussi des chants obscènes, loin des oreilles masculines, lorsqu'elles coupent et transportent les herbes pour la construction des cases ${ }^{1}$.

Chez les Lugbwara d'Uganda, à la saison des plantations, les hommes exécutent une danse spéciale accompagnée de chants érotiques. Durant cette danse, ils arrivent délibérément à une semi-érection du pénis. Parvenu à ce stade, chaque individu se retire rapidement de la danse, y revient une fois le pénis devenu normal, puis répète sa performance.

J'ajouterai une remarque inédite provenant de mes propres recherches sur les Ingassana de la province Fung du Soudan anglo-égyptien. Ce peuple possède une association spéciale d'individus qui dansent et chantent lors des mariages, de la naissance des jumeaux et de la maladie des enfants. Ils accompagnent leurs exhibitions de petits jeux obscènes avec un

Pour une étude sur les Didinga, cf. DRIBERG, People of the Small Arrow, et Didinga Customary Law, Sudan Notes and Records, 1925. 
phallus en bois. Les chants aussi sont probablement licencieux, en accord avec le caractère sexuel de ces cérémonies.

\section{VII - Résumé des données et formulation du problème}

Retour à la table des matières

Les données de Driberg et les nôtres, collectées en Uganda et au Soudan anglo-égyptien, complètent notre analyse de l'obscénité chez les Ba-Ila, les Ba-Thonga, les Akamba et les Azande.

Il faut, tout d'abord, faire ressortir que ces manifestations d'obscénité sont habituellement interdites. Les gens les considèrent comme tout à fait déplacées et elles sont absolument prohibées en dehors des occasions signalées. Elles appartiennent à ce domaine de la vie mentale que la société stigmatise comme grossier et vulgaire et qui doit être refoulé par l'individu. En de rares occasions, cependant, la société, non seulement autorise, mais prescrit ces exhibitions. L'analyse de ces occasions conduit à l'explication fonctionnelle des types de conduite érotique mentionnés dans cet article.

Certaines circonstances, que nous pouvons décrire comme magico-religieuses, autorisent l'obscénité. Ce sont :

- les cérémonies d'initiation (Ba-Ila, Ba-Thonga, Akamba, Lango, Didinga);

- les cérémonies funéraires (Ba-Ila, Ba-Thonga);

- les festins en l'honneur des esprits (Ba-Ila);

- les cérémonies de la pluie (Ba-Thonga, Akamba, Lango) ;

- la cérémonie pour protéger les récoltes des insectes nuisibles (Ba-Thonga);

- le vol aux dépens de l'oncle maternel (Azande);

- les cérémonies des sociétés secrètes (Azande, Wapare, etc

- les cérémonies pour la naissance des jumeaux (Lango, Ingassana);

- le mariage et la maladie des enfants (Ingassana);

- la cérémonie pour protéger les récoltes (Didinga);

- la danse des semailles (Lugbwara).

Le second type de circonstances où l'obscénité est admise se caractérise par un travail collectif long et pénible. Il est impossible de distinguer clairement entre les deux groupes, car beaucoup d'occasions que nous avons considérées comme rituelles ont un aspect économique, par exemple les cérémonies pour faire tomber la pluie. Bien des travaux s'intègrent dans un cycle de cérémonies, tels le battage et la mouture du mil chez les Azande. Si nous employons l'adjectif « rituel » dans un sens très large, alors toutes les occasions d'expression d'obscénités présentées dans cet article peuvent être classées comme « occasions rituelles ». Mais il serait préférable de proposer un sous-groupe où seraient incluses les 
obscénités qui accompagnent les travaux : les semailles, la fonte du fer, la pêche, la mise à l'eau des pirogues (Ba-Ila), le pilage du maïs, le transport des toits, le bousillage des sols (BaThonga), le battage, la mouture, le transport des pierres lors d'un travail pour le compte du gouvernement (Azande), la construction des cairns (Didinga), la coupe et le ramassage des herbes (Didinga).

Tout en essayant d'expliquer ces obscénités, je tenterai d'abord d'envisager pourquoi les interdits de la société se relâchent dans les circonstances précitées. Je me propose ensuite de définir le but de ce relâchement dans certaines cérémonies et son rôle économique dans le travail. Je poursuivrai ainsi l'analyse effectuée à partir des données collectées, car je crois que la méthode adéquate d'interprétation d'un élément culturel consiste à le décrire en termes généraux, auxquels on se réfère pour expliquer comment il intervient en tant qu'élément spécifique dans la vie d'une société. Cet élément culturel se rencontre simultanément dans de nombreux contextes différents, associé à des éléments différents, dont on ne peut saisir la signification que par l'analyse de chaque contexte spécifique. En conséquence, un élément comme celui que nous avons décrit peut avoir un nombre indéfini de fonctions sociales, mais il possède une caractéristique fonctionnelle commune à toutes.

Avant de fournir notre propre explication, nous nous attacherons à celles proposées par deux des auteurs auxquels nous avons emprunté la majeure partie de nos données pour cet essai.

Notre intention est d'expliquer comment des actions normalement tabou sont en certaines occasions des modes d'expression collective imposés.

\section{VIII - Fonction sociale de l'obscénité collective}

\section{$\underline{\text { Retour à la table des matières }}$}

Comment expliquer que l'obscénité soit de règle en certaines circonstances ? Smith et Dale proposent une explication basée sur la considération quelque peu mystique du prétendu dynamisme des Ba-Ila, terme par lequel ils désignent toutes les idées magiques et religieuses de ce peuple. Ils nous déclarent que l'on exécute les chants, que l'on doit les exécuter, lorsque les forces dynamiques entrent intimement en jeu. Ils concluent: «En temps ordinaire, l'anormal est tabou, mais aux circonstances exceptionnelles correspondent des choses exceptionnelles dont le but est de restaurer l'équilibre habituel » (Il, p. 84).

Cette explication, tout comme celle du dynamisme, semble quelque peu mystique, et je ne saisis pas la conclusion, car je ne considère pas la mise à l'eau d'une pirogue, les semailles, la pêche, comme des circonstances exceptionnelles, même si elles sont saisonnières. 
La théorie de Junod et apparemment celle de Lindblom s'inspirent des études de Van Gennep dans Les rites de passage. Suivant cette théorie, les chants obscènes sont justifiés par le fait qu'on les chante lors de périodes «marginales » qui interviennent entre des rites de « ségrégation » et des rites d' " agrégation ». Ainsi, par exemple, la construction d'un village représente chez les Ba-Thonga une période «marginale » d'un mois ou plus, durant laquelle les lois ordinaires sont suspendues et de nombreux tabous particuliers renforcés. Ces actes et ces chants obscènes ont pour but de mettre en relief la suspension des lois ordinaires durant les «périodes marginales » des rites de passage. C'est pourquoi des occasions semblables entraînent une obscénité semblable dans les rites de passage de l'initiation et des funérailles.

La généralisation de cette explication soulève des objections évidentes, car elle ne s'applique qu'à quelques cas parmi ceux présentés dans cet article. Les cérémonies de la pluie et du пипи que décrit Junod ne peuvent être classées comme rites de passage, tout au moins au sens admis de l'expression. Le battage de l'éleusine et la mouture du grain chez les Azande, les semailles, la pêche, les cérémonies du makubi chez les Ba-Ila ne sont certainement pas des rites de passage.

Ainsi, nous ne pouvons admettre la théorie selon laquelle ces obscénités auraient pour fonction de recréer ces conditions normales lors d'une situation anormale (Smith et Dale), ni celle directement opposée selon laquelle la fonction des obscénités serait de créer une période marginale entre des rites de « ségrégation » et des rites d' « agrégation » (Junod).

Cependant, nous conserverons présentes à l'esprit les observations de ces auteurs : l'obscénité se situe souvent en périodes exceptionnelles, comme la sécheresse, et souvent en périodes de transition, comme l'initiation, mais nous essaierons d'atteindre à une explication plus générale que la leur.

L'explication que j'avance pour rendre compte de ces expressions spécifiques de l'obscénité est très simple. A mon sens et sous un certain angle, il y a identité de fonction sociale entre le tabou, en tant qu'interdit spécifique, et ces expressions de l'obscénité.

Qu'est-ce qu'un tabou ? Selon la terminologie des religions de Notes and Queries on Anthropology, "un tabou se limiterait à décrire un interdit lié à une sanction magicoreligieuse ». En outre, «d'autres interdits sont observés dans les sociétés archaïques, les interdits légaux imposés par l'autorité et les interdits coutumiers qui ne reposent que sur la désapprobation sociale, mais le terme tabou doit être réservé au cas défini ci-dessus » (p. 262, 4e éd.).

Plusieurs raisons rendent ce mode de définition peu satisfaisant. Par exemple, selon l'état du développement politique de chaque société, des interdits exactement semblables seront, dans des sociétés différentes, classés dans des catégories différentes. Il est toujours difficile de déterminer l'origine de la sanction d'un interdit. Pour un même interdit, elle peut s'appuyer sur la désapprobation sociale, sur l'autorité politique aussi bien que sur les forces magicoreligieuses. Où classerons-nous la prohibition de l'inceste ? Sa sanction est-elle d'origine magico-religieuse, provient-elle de l'autorité, ou repose-t-elle simplement sur la désapprobation sociale? Tout dépend du développement spécifique de chaque société. 
Je ne pense pas qu'un tabou doive être traité comme une entité séparée, définie par sa sanction, mais je suis d'avis de le considérer toujours comme un interdit faisant partie intégrante d'une activité sociale. Sa sanction devrait généralement dépendre de l'activité sociale elle-même, si un tabou alimentaire ou un tabou sexuel est transgressé avant la pêche, celle-ci ne sera pas fructueuse; si un tabou est transgressé avant l'exécution de rites magiques, la magie restera sans effet.

On ne tient habituellement pas compte du fait que, chez les indigènes, le tabou se place au premier rang des préoccupations, et que son importance découle non pas de l'interdiction d'une chose ou d'une autre, mais de ce que cette chose ou cette autre font partie de la vie quotidienne, d'une activité routinière, d'une habitude, par exemple, les tabous portant sur les rapports sexuels, sur l'alimentation ou sur la boisson. C'est parce que l'indigène n'est pas autorisé à se comporter comme de coutume que son attention est centrée sur l'activité à laquelle s'attache l'interdit. De cette façon, se fixe dans l'esprit de l'individu l'importance des activités à valeur sociale. Je considère que c'est là une des fonctions essentielles du tabou.

Nous pouvons envisager sous le même angle les interdits relatifs aux personnes. Les relations d'un homme avec sa sœur sont mises en relief, moins par une impossibilité de rapports sexuels que parce qu'elle est la seule partenaire qui lui soit interdite. Pour une raison semblable, les relations d'un homme avec sa belle-mère prennent un caractère exceptionnel $\mathrm{du}$ fait que, de toutes les femmes, elle est la seule qu'il ne puisse rencontrer et à qui il ne puisse parler. Les attitudes à l'égard du chef sont souvent réglées par de semblables tabous, par exemple en Nouvelle-Zélande et à Samoa. Cependant, dans cet article, mon intention est moins de parler des tabous que des dérogations aux interdits. De même que la tradition de répétition d'une activité spécifique renforce le tabou, de même la tradition contrôle socialement et règle les circonstances du comportement obscène. Tout comme la caractéristique principale du tabou est d'interdire à l'individu d'accomplir ce qui est habituellement permis, la caractéristique principale de l'obscénité est d'autoriser l'individu à faire ce qui est normalement prohibé. Une fonction commune du tabou et des comportements obscènes est de rompre la routine quotidienne et de mettre ainsi en relief la valeur sociale ${ }^{1}$ de l'activité à laquelle ils sont liés.

Un exemple Ba-Ila rapporté par Smith et Dale illustre bien le rôle semblable du tabou et des comportements obscènes. Pour un Ila, rien n'est plus affreux que les rapports d'un homme avec sa sœur ou avec sa fille, pourtant: «Si un individu souhaite s'attirer la chance, non seulement il recourt aux fétiches, mais suivant les prescriptions de son guérisseur, il doit avoir des rapports incestueux avec sa sœur ou avec sa fille avant de se lancer dans son entreprise » (I, p. 261). Nous nous trouvons face à cette conduite sacrilège au moment où nous aurions cru que les tabous sexuels et alimentaires se seraient alliés à la magie. Chez les Ba-Thonga, à la mort d'un homme, ses femmes se prostituent rituellement, alors que, dans une telle circonstance, on aurait pu s'attendre à de sévères tabous sexuels.

Je pense que l'explication proposée nous aidera à comprendre les comportements d'obscénité obligatoire et stéréotypée. Elle ne rend pas compte de tous les types d'obscénité et n'essaie pas d'en éclairer la psychologie. Elle ne vise qu'à expliquer pourquoi l'obscénité collective, socialement contrôlée et stéréotypée, est associée à certaines activités sociales.

Pour le concept de 4 valeur sociale », cf. A. R. RADCLIFFE-BROWN, Andaman Islanders, passim, 


\section{IX - Relation entre l'obscénité et les actes rituels}

$\underline{\text { Retour à la table des matières }}$

J'ai déclaré plus haut que le tabou on pourrait dire la même chose de l'obscénité stéréotypée ne devrait pas être traité comme une entité distincte définie par sa sanction, mais qu'il devrait être considéré en relation avec l'activité dans laquelle il joue un rôle. Il nous a donc été possible d'aboutir à une généralisation qui explique la relation de l'obscénité stéréotypée en général, avec des activités de valeur et d'importance sociales. Il nous faut encore montrer son rôle spécifique dans des activités spécifiques.

Nous avons remarqué que les obscénités collectives font généralement partie d'importantes cérémonies, telles que celles qui accompagnent un décès, la naissance des jumeaux, la sécheresse, le passage initiatique à l'état d'homme. Toutes ces circonstances contiennent une terrible force émotionnelle très dangereuse pour l'individu qui les vit et pour la société. Les émotions refoulées de colère, de peur, de chagrin, de douleur, atteignent un tel degré qu'elles ont besoin de s'exprimer dans une activité ; à moins que cette activité ne soit canalisée dans des directions inoffensives, elle peut être dommageable à l'individu et cause de bouleversement pour la société. En de telles circonstances, la société absout et même ordonne des actes qu'elle interdit et pénalise habituellement.

On sait cependant que ces inclinations élémentaires, sexuelles par exemple, essentielles au maintien de la société aussi bien qu'à celui de la vie individuelle, n'en sont pas moins, dans leur expression incontrôlée, incompatibles avec la forme de culture la plus primitive qui nous soit connue. Pour préserver et transmettre la culture, les satisfactions individuelles doivent être subordonnées aux fins sociales, ce qui contraint l'individu à bien des expériences pénibles : le travail, l'obéissance à une autorité, la limitation des désirs par les conventions sociales, le respect de nombreux interdits et prescriptions qui modèlent et transforment les inclinations communes de l'espèce (les instincts). Tous les systèmes sociaux, aussi variés que complexes, semblent s'être développés comme le nôtre ; les systèmes supérieurs comme ceux que nous avons construits -science, art, religion - retrouvent tous leur source énergétique dans ces inclinations élémentaires, sexuelles particulièrement. Par un processus, auquel les psychanalystes ont donné le nom de sublimation, les inclinations les plus défavorables au développement de la culture sont devenues les sources mêmes qui l'ont alimenté.

Un tel processus peut cependant manquer son but. Une répression trop brutale de ces inclinations élémentaires ne conduit pas l'individu à mettre ses forces au service d'une fin culturelle, mais provoque chez lui des obsessions, des désillusions, le suicide parfois, si bien 
qu'il n'est en rien utile à la société. Freud et son école sont à l'origine de cette vision de la culture, et ils ont mis en évidence comment certaines sociétés laissent, dans une certaine mesure, libre cours à ces inclinations élémentaires, et comment la nôtre devrait les imiter, ce qui serait beaucoup moins dangereux que de les réprimer brutalement.

J'ai jugé bon d'attirer l'attention sur cette vaste théorie de la culture afin que notre explication de l'obscénité lors des cérémonies rituelles ne soit pas isolée, mais qu'elle s'intègre dans un ensemble de coutumes sociales à fonction similaire, à savoir, canaliser de façon inoffensive l'activité occasionnée par une tension émotionnelle dangereuse pour l'individu et cause de bouleversement pour la société.

Quelques exemples nous aideront à comprendre. Parmi de nombreuses tribus, les cérémonies d'initiation des garçons et des filles sont l'occasion d'obscénité. Le trait commun à ces initiations (ainsi qu'à celle des sociétés secrètes) est la rigueur avec laquelle on traite les initiés, lesquels doivent supporter de sévères flagellations, des tracasseries, des tourments, la faim, la soif, un travail fatigant, des danses épuisantes, d'âpres humiliations, le viol, la sodomie et beaucoup d'autres expériences. Non seulement leur mode de vie provoque une tension émotionnelle, mais les sévérités, les souffrances, la fatigue tendent à conduire les individus à rechercher une compensation immédiate, probablement d'ordre sexuel ${ }^{1}$.

La sévérité des cérémonies d'initiation fait essentiellement partie de leur but éducatif, qui ne saurait être atteint si elles ne conduisaient qu'à des passions soudaines et intempestives. L'existence pour l'initié d'un secteur d'activités autorisées, alternant avec ses expériences pénibles et fatigantes, contrebalance celles-ci, tout en conservant son but à la discipline infligée.

Si nous prenions chaque occasion type où s'exprime l'obscénité collective : la mort, la naissance des jumeaux, la sécheresse, etc., nous pourrions montrer que chacune d'entre elles, comme l'initiation, provoque chez l'individu une grande tension émotionnelle, dont l'expression n'est pas laissée au hasard, mais déterminée par la société et canalisée par la tradition.

Nous n'avons cependant pas l'intention de considérer séparément chaque cas, les conclusions ci-dessus fournissent une explication psychologique valable pour tous. Nous ajouterons donc un appendice à nos généralisations :

L'obscénité collective et imposée a pour fonction générale la mise en évidence de la valeur sociale de l'activité à laquelle elle est liée.

La plupart des occasions entraînant ce type d'obscénité représentent des phases critiques de la vie humaine, dangereuses pour l'individu autant que pour la société. L'autre fonction de l'obscénité est donc de fournir un moyen socialement autorisé d'exprimer cette émotion.

«Lorsque, par exemple, la réalité est trop pénible, la vie trop difficile à supporter, les activités mentales de l'individu ont tendance à retourner à leurs formes élémentaires avec toutes les conséquences qui en découlent » (JONES, Papers on Psycho-analysis, p. 5). 


\section{X - Fonction économique de l'obscénité}

\section{$\underline{\text { Retour à la table des matières }}$}

Nous avons noté que l'obscénité, en particulier les chants obscènes, accompagne souvent un travail collectif déterminé. Avant d'expliquer cette relation spécifique, j'attirerai l'attention sur une autre, celle existant entre les tabous et les travaux à caractère économique.

Tout travail est désagréable et réclame une impulsion ${ }^{1}$. Les impulsions proviennent d'incitations, de stimuli concomitants, de la participation d'autres personnes et de divers facteurs avec lesquels les économistes nous ont familiarisés. Ces impulsions se révèlent particulièrement nécessaires lors d'un travail collectif long et pénible. Dans cette entreprise d'ordre économique, le tabou agit comme élément d'impulsion. Je n'ai pas encore cité d'exemples de tabous associés aux diverses entreprises précédemment décrites, aussi en présenterai-je maintenant deux.

Lorsque les Ba-Thonga désirent construire un nouveau village, ils rassemblent d'abord les matériaux de construction. Puis, avant de commencer à bâtir les cases, le chef quitte l'ancien village qu'il ne reverra peut-être jamais. Il emmène sa première femme, et, le soir, ils ont des rapports sexuels à l'endroit choisi pour le nouveau village. Le lendemain, le reste du village arrive. Alors débute une période de déménagement d'environ un mois (buhlapfa). Pendant ce mois, les travailleurs doivent respecter certains tabous - prohibition absolue des rapports sexuels, défense de se laver. Les motifs invoqués pour expliquer ces tabous sont les suivants : les relations sexuelles nuiraient au chef du village, les ablutions feraient tomber la pluie. D'autres tabous s'attachent également à ce buhlapfa. Il est interdit d'allumer du feu dans le nouveau village avant son achèvement, la cuisine doit se faire à l'extérieur ; il est aussi défendu de piler le maïs, de danser, de siffler, ce qui attirerait les sorciers (1, p. 306 sq.).

Cet exemple montre bien le rôle du tabou dans le travail. Les plaisirs sexuels, ceux de la toilette et de la danse, la cuisine à l'intérieur du village sont interdits, la routine quotidienne de la vie familiale est interrompue jusqu'à l'achèvement de la tâche.

Voici un autre exemple. J'ai déjà mentionné la fonte du fer chez les Ba-Ila. Lors de cette longue et difficile opération, les ouvriers vivent loin de leurs foyers. Tandis qu'ils habitent dans leurs abris temporaires, «si l'un d'entre eux désire se rendre au village, il ne doit en

Il n'est pas nécessaire d'épiloguer sur le désagrément du travail et sur la nécessité de surmonter la répugnance qu'il inspire. Il est cependant intéressant de noter l'étymologie du mot « travail ». Le mot français travail s'apparente à l'italien travaglio qui signifie «souffrance »; au Moyen Age, on utilisait le mot «douloureux » dans le sens de « laborieux P; le mot italien lavoro vient du latin labor : douleur ; quant au mot grec [mot en grec] il signifie à la fois «travailler » et «souffrir », tout comme l'hébreu assab (JONES, ibid., p. 175). 
aucun cas avoir des rapports avec sa femme. Il n'a pas le droit d'entrer chez lui, en particulier, il ne doit pas s'asseoir sur son lit, mais s'accroupir à la porte de sa case ; là, il peut manger, si sa femme lui prépare quelque chose. Quant aux femmes qui demeurent au village, elles n'ont le droit ni de se laver, ni de se parfumer, ni de porter des parures qui pourraient attirer les hommes. Elles ressemblent à des femmes au veuvage récent ». Tandis qu'ils fabriquent les fours, les hommes ne doivent pas boire de l'eau, mais uniquement du namenze (1, p. 207).

De nouveau, nous remarquons comment les hommes sont privés du confort et des plaisirs de la vie au foyer jusqu'à l'accomplissement du travail, et comment les femmes ont interdiction d'user de tout ce qui pourrait favoriser les désirs sexuels nuisibles à la société.

Pour étudier la fonction de l'obscénité, j'emploierai la méthode utilisée pour examiner le rôle du tabou dans une activité d'ordre économique.

Nous savons que l'obscénité stéréotypée, spécialement les chants obscènes, accompagnent certains travaux spécifiques. Malheureusement, de ce point de vue, nos connaissances sur les Ba-Ila, les Ba-Thonga et d'autres, manquent de précision pour que nos conclusions atteignent à une parfaite certitude. Les chants obscènes ont une signification rythmique et érotique. J'analyserai d'abord l'aspect rythmique.

J'ai montré que, chez les Azande, ces chants impriment leur rythme au travail. Lorsqu'elles battent le mil ou broient le grain germé, toutes les femmes exécutent les mêmes mouvements sur un rythme rapide. Les Ba-Ila accomplissaient probablement les semailles, la pêche, la fonte du fer, la mise à l'eau des pirogues au rythme de leurs chants érotiques, mais nos sources ne sont pas suffisamment explicites. A défaut de précision, on peut supposer que les Ba-Thonga n'auraient pas pu transporter le toit de leurs cases et piler le maïs en chantant, à moins que leurs mouvements n'aient suivi, jusqu'à un certain point, le rythme de leurs chants.

La coordination du travail et du rythme est un sujet important pour les théoriciens. Il est bien connu que le travail rythmique diminue la fatigue ${ }^{1}$. La valeur fonctionnelle du rythme s'accroît lors d'un travail collectif, car il permet de coordonner harmonieusement les mouvements des travailleurs qui participent à l'opération.

Je ne m'attarderai pas davantage sur ce sujet maintes fois repris, car, plus tard, j'espère traiter plus longuement des relations du rythme et du travail chez les Azande.

Les chants décrits ci-dessus ne se caractérisent pas seulement par leur rythme, mais aussi par leur signification érotique. On peut considérer le privilège accordé aux travailleurs de chanter des chansons vulgaires, condamnées comme choquantes et indécentes en temps ordinaire, comme un des palliatifs à la lassitude et à la monotonie du travail. Quiconque a observé les indigènes battre le mil ou moudre le grain pendant des heures, sait combien ce travail est épuisant. Quiconque a vu des hommes transporter le toit d'une case sur une longue distance, ou bien une femme piler le grain, sait combien la première tâche est pénible et la 
seconde monotone. Les palliatifs offerts aux travailleurs leur permettent d'accomplir plus efficacement les travaux fatigants : transport de pirogues, semailles, pêche, fonte du fer ${ }^{1}$.

\section{XI - Conclusion}

$\underline{\text { Retour à la table des matières }}$

Résumons nos conclusions :

1) Certains types de comportements obscènes uniquement collectifs sont habituellement interdits, sauf en certaines circonstances où ils sont autorisés et même prescrits.

2) Ces circonstances de grande importance sociale se classent, grosso modo, en deux groupes : les cérémonies religieuses et les activités collectives d'ordre économique.

Nous expliquons l'obscénité de la façon suivante :

1 Seligman a souligné la «dramatisation » de ces expressions sociales de l'obscénité. Il a aussi attiré mon attention sur l'explication psychanalytique des données de cet article. Ma propre explication de la relation obscénité-travail s'accorde bien avec la théorie psychanalytique selon laquelle la fonction de l'obscénité est d'agir comme une impulsion et un palliatif du travail, et selon laquelle les actes d'obscénité résultent d'un heurt entre la nécessité du travail (principe de réalité) et le désir d'éviter l'épuisement (principe de plaisir). Cependant les psychanalystes développent leur thèse en montrant que le même mot-symbole désigne à la fois le travail et l'accouplement, non seulement parce que certains de leurs attributs sont Semblables, si bien que les deux activités distinctes se trouvent associées dans l'esprit, mais parce qu'il y a une raison spécifique à l'extension du terme initial d'accouplement pour désigner son équivalent symbolique, le travail. D'après ces auteurs (Cf. JONES, ibid., p. 177, et SPERBER, Imago, 1912, « über den Einfluss sexueller Momente auf Entstehung und Entwicklung der Sprache »), l'homme primitif dépasse son inertie et sa répugnance pour le travail en sexualisant ses tâches. Il les traite comme 4, un équivalent ou un substitut de la fonction sexuelle ». J'avoue douter de la signification donnée à leur terminologie, par les auteurs de cette école. Que veulent-ils dire quand ils écrivent que les tâches sont des «équivalents » ou des « substituts » des actes sexuels ? On peut admettre que, chez les peuples primitifs, une grande partie de l'énergie consacrée au travail le serait au plaisir sexuel. Il est probablement vrai aussi que l'intérêt sexuel stimule le travail. Mais, s'il en est ainsi, 19, fatigue provoquée par un travail pénible et épuisant devrait conduire à une tentative de soulagement dans le plaisir sexuel; or celui-ci est impossible, puisque le désir sexuel est sévèrement réprimé pour permettre l'achèvement de l'entreprise. Certes, un mode alternatif de détente s'offre alors sous forme de symbolisme érotique et de chants, mais je ne comprends pas par quel processus le travail est ainsi sexualisé ou, plus précisément, je ne comprends pas la signification d'une telle affirmation. Je préfère simplement faire état de la théorie. Je ne pense pas que soit très fort l'intérêt sexuel consciemment lié à l'obscénité. A mon avis, son importance découle, non pas de son caractère sexuel, mais de la suppression de l'interdit habituellement imposé par la société : cette suppression constitue pour les travailleurs un remède tonifiant. 
1) L'annulation, par la société, de certains interdits met en relief la valeur sociale de l'activité.

2) Elle canalise l'émotion humaine vers des formes d'expression inoffensive aux moments critiques.

3) Elle offre aux travailleurs un stimulant et une compensation lors des travaux collectifs pénibles et d'une certaine manière, par exemple avec des chants, elle coordonne les activités. 


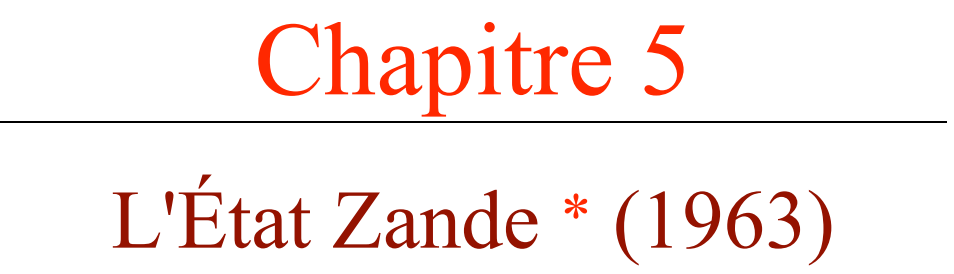

Retour à la table des matières

Je consacrerai cette conférence à l'étude des institutions politiques azande. La théorie anthropologique classique reposait en grande partie sur des recherches effectuées parmi des peuples dépourvus d'institutions politiques développées, et pendant longtemps, on n'accorda pas, à l'organisation politique des peuples primitifs, l'attention qu'elle méritait. Les sociétés africaines en particulier présentent une gamme relativement étendue d'institutions politiques, et déjà l'étude de ce secteur de l'anthropologie sociale a beaucoup progressé. En prenant pour exemple les Azande, j'ai l'intention de résumer les conclusions générales sur la nature de leurs institutions politiques, et ce faisant, d'attirer l'attention sur la complexité des matériaux qu'offrent les sociétés du même type, et sur la nécessité, en les étudiant, de prendre en considération les données historiques disponibles. Pour certaines sociétés, de telles données font défaut, ou, si elles existent, elles montrent combien peu de changements sont intervenus durant la période en question. Mais, chez les Azande, la situation politique, au moins sous certains de ses aspects importants, n'avait pris que depuis peu la forme qu'observèrent les premiers voyageurs européens, et elle devait subir d'autres changements dans les années à suivre. Au cours de ce survol, il m'est difficile d'éviter de me référer fréquemment à ma

Cette conférence fut prononcée à la demande du Royal Anthropological Institute, le 23 juin 1963, et publiée dans le J.P.A.I. en 1963. 
propre recherche des années 1927 à 1930, principalement sur le vieux royaume de Gbudwe au Soudan. Quant aux observations des autres anthropologues, je leur ai accordé la considération qu'elles méritaient dans mes publications antérieures citées en référence.

Une description des institutions politiques azande réclame, dans une certaine mesure, une reconstitution, car si la vie sociale en général, autant qu'on Puisse l'affirmer, ne s'est guère modifiée après l'installation de l'administration européenne, il n'en a pas été de même des institutions politiques. Le pouvoir des princes régnants s'affaiblit, de même que les privilèges et services qui en dépendaient. On maintint les cours, mais les compagnies militaires qui y étaient attachées disparurent. Le tribut en nature et en travail diminua, et, par voie de conséquence, l'hospitalité de la Cour. On consultait encore les oracles des princes, mais les officiers européens n'y attachaient aucune considération, ils étaient les seuls à déterminer les sanctions. Les princes gouvernaient les provinces comme auparavant, mais le gouverneur suprême, le roi, à qui ils juraient autrefois allégeance, avait été remplacé par le commissaire de district. Cependant, si bien des coutumes avaient disparu, beaucoup demeuraient, qui suffisaient pour permettre d'observer le système traditionnel, même partiellement décadent. Dans la région où j'ai effectué mes recherches, l'implantation administrative avait été lente jusque dans les années récentes.

Mes informateurs étaient donc pour la plupart des gens élevés dans le vieux système dont ils conservaient un vivant souvenir, Ils avaient vécu en contact avec les cours des rois et des princes, avant l'ère européenne. Les schèmes de pensée peuvent subsister même lorsque les institutions qui les ont modelés ont perdu de leur actualité. Les traditions orales concernant l'histoire nous furent utiles aussi, d'autant que, comme nous, les Azande prennent la succession de leurs gouvernants comme point de repère historique. Les récits de valeur inestimable fournis au début du siècle par de Calonne-Beaufaict (1921), Hutereau (1909 ; 1922) et Czekanowski (1924), puis plus tard par Mgr Lagae (1926), Vanden Plas (1921), le major Larken $(1926$; 1927) et d'autres, m'apportèrent une aide supplémentaire qui me permit de reconstituer le système politique, confirmant ainsi les traditions historiques. Ces indications nous reportent soixante ans en arrière. Et nous avons encore la chance de bénéficier d'autres informations. Durant la deuxième moitié du siècle dernier, nombre de voyageurs européens visitèrent le pays zande. Trois d'entre eux, Piaggia, Junker et Casati y ont résidé longtemps, et nous ont laissé, de même que Schweinfurth, de longues descriptions sur le mode de vie zande (Antinori, 1868 Schweinfurth, 1873 ; Junker, 1890 ; 1891 ; 1892 ; Casati, 1891 Piaggia, 1941). Sans doute faut-il utiliser les premiers récits avec un prudent esprit critique. On peut s'interroger sur la validité des témoignages, comme j'ai essayé de le faire dans mon article sur «Le cannibalisme zande » (Evans-Pritchard, 1960 a) ${ }^{1}$. Mais ils nous apportent, sur les conditions politiques qui prévalaient à l'époque, des renseignements importants et certainement exacts dans l'ensemble. Pour l'essentiel, ils confirment aussi les traditions consignées par des observateurs beaucoup plus récents. Ces récits nous reportent un siècle en arrière, mais dans la mesure où ils s'appuient sur des traditions orales de l'époque où ils furent écrits, ils nous offrent une connaissance de l'histoire zande remontant jusqu'à la première moitié du XIXe siècle et môme jusqu'au XVIIIe

Les caravanes arabes commencèrent leur commerce d'ivoire et d'esclaves en pays zande aux environs de 1860 , ce qui dans certaines régions provoqua immédiatement des troubles.

Chapitre suivant de cet ouvrage. 
Vers 1870, augmentant la confusion, arrivèrent les forces du gouvernement égyptien qui ne furent pas sans ajouter à la cruauté et au chaos de l'époque, lors du renversement de leur gouvernement par les Derviches au Soudan. A l'extrémité sud, un quatrième groupe d'Arabes, commerçants et marchands d'esclaves, venus de Zanzibar et de la côte est-africaine, ne firent qu'accroître le désordre. Les royaumes azande, plus organisés que certains peuples des alentours, purent mieux résister aux envahisseurs, et éviter ainsi l'exploitation et l'esclavage auxquels furent soumis quelques-uns de leurs voisins. Mais leur histoire se compliqua avec les entreprises des Arabes. Aux rivalités et aux guerres dynastiques, s'entremêlèrent des forces et des événements différents de par leur origine et de par leur évolution. A la fin du siècle, les Européens arrivèrent: les Français et les Belges par l'ouest, et plus tard les Britanniques par le nord. Apparemment, ils combattaient les Derviches, mais chaque grande puissance essayait aussi d'étendre ses possessions africaines aux dépens des autres puissances, et les Azande se trouvèrent inévitablement impliqués dans cette conjoncture. Leur territoire fut finalement conquis et divisé entre les trois Empires coloniaux. Le dernier royaume à préserver son indépendance, celui de Gbudwe, la perdit en 1905.

Longtemps avant que l'arrivée des Arabes et des Européens ne vienne ajouter au désordre, régnait une situation confuse et troublée. Guerres et querelles dynastiques abondent dans l'histoire zande. Probablement vers le milieu du XVIIIe siècle, une partie considérable des Ambomu, sous la conduite de leur maison royale Vongara, quittèrent leur pays d'origine, les vallées des rivières Mbomu et Shinko, maintenant en République centre-africaine, et se dirigèrent vers le sud-est, c'est-à-dire vers l'ex-Congo belge, puis vers l'est, le sud et enfin vers le nord, pour s'installer au Soudan. Au cours de ces migrations, ils conquirent de vastes territoires, repoussant ou assujettissant un certain nombre de peuples étrangers dont les descendants s'assimilèrent plus ou moins aux conquérants ambomu pour former le peuple zande d'aujourd'hui. Aux guerres presque incessantes contre les peuples étrangers s'ajoutaient les guerres entre les rois et les princes avongara pour le partage du butin.

J'analyserai brièvement quelques-unes des conséquences de cette expansion, mais, avant de le faire, je voudrais mettre en évidence, qu'étant donné la diversité des expériences historiques dans les différentes régions azande, ce serait un travail gigantesque d'écrire l'histoire complète des Azande. Selon diverses appréciations, on a estimé que leur nombre variait de 750000 à deux ou trois millions et même plus. On peut considérer qu'en gros, ils occupent un territoire allant de 150000 à 250000 kilomètres carrés, qui s'étend de la savane au nord à la forêt tropicale au sud. Écrire l'histoire de ce peuple revient donc à écrire un ensemble d'histoires locales. Chaque région connaît sa propre histoire et ignore celle des autres. Elle l'ordonne autour de la succession de ses rois, mais aucun système de dates ne nous permet de relier les événements d'un royaume avec ceux d'un autre. Dans une certaine mesure, de Calonne a entrepris ce travail auquel j'ai ajouté ma contribution, avec une courte histoire du royaume de Gbudwe (Evans-Pritchard, 1956 a) et une série de textes vernaculaires sur les événements historiques et les institutions politiques (Evans-Pritchard, 1955-1957). Cependant, en dépit de la diversité écologique et historique, il semble que les institutions politiques aient été partout fondamentalement semblables.

Une des conséquences de ces guerres, de ces mouvements et de ces migrations parait être l'accroissement de la puissance des Avongara. Les Azande eux-mêmes affirment que la guerre et l'apport de nouveaux éléments lors de chaque conquête renforçaient l'autorité de leurs chefs. Une autre conséquence fut la complexité des brassages ethniques. J'ai recensé 
plus de vingt peuples (à défaut d'informations complètes, une plus grande précision est impossible) dans l'amalgame zande, dont bien des individus appartiennent encore à d'autres groupes étrangers (Evans-Pritchard, 1958 a). La détermination certaine des origines ethniques s'avère difficile, mais il est probable qu'à l'exception de la région gouvernée par la dynastie des Yakpati, les individus d'ascendance étrangère dépassent aisément le nombre des Ambomu, et même dans cette région où la plus grande partie des Ambomu sont supposés avoir émigré, le recensement d'environ 33000 à 34000 habitants révèle que $29 \%$ seulement des personnes recensées sont des Ambomu. Certains peuples étrangers forment encore, sous l'hégémonie vongara, des communautés parlant, en même temps que le zande, leur propre langue : le soudanais, le bantou et le nilotique. Au Soudan seulement, dans la région zande, on parle sept ou huit langues différentes en plus du zande. Avant que les Arabes ne refoulent les Azande vers le sud, on en comptait encore davantage. Lors de mon séjour sur le terrain, des anciens parlaient encore d'autres langues autrefois utilisées par des peuples entiers maintenant dispersés dans cette phase finale d'assimilation culturelle. Ces éléments étrangers présentent tous les stades d'intégration allant de la fusion politique avec autonomie culturelle jusqu'à l'assimilation complète, politique et culturelle.

Nous noterons quelques résultats de ce processus. Là où la majorité était étrangère, comme dans les régions bordant les zones d'expansion, la maison régnante semble avoir été plus autocratique, plus distante et avoir exigé une soumission que les Ambomu n'auraient pas acceptée. L'extension d'une politique colonisatrice en fut une autre conséquence. Les Avongara avaient pour politique, non seulement de laisser un peuple soumis sur son territoire, mais aussi de confier son gouvernement à ses propres chefs, n'exigeant que la seule reconnaissance de leur suprématie et un tribut en prestation de travail et en biens. Ainsi les principaux citoyens ambomu ou d'ethnies assimilées étaient-ils encouragés à s'établir en territoire conquis, ce qui occasionnait une plus grande dispersion et un plus grand brassage des clans. Ces colonisateurs, leurs parents et leur suite formaient des noyaux à partir desquels se répandaient le langage, les habitudes, les coutumes et les institutions politiques, jusqu'au moment où, à l'exception des régions où ils constituaient de vastes communautés géographiquement isolées, les étrangers se confondaient avec les Ambomu et finissaient par se considérer avec ceux-ci comme des Azande. A un certain moment, une branche de la maison royale établissait sa Cour au milieu de ces étrangers, les encourageant à participer plus largement aux affaires politiques, accélérant ainsi le processus d'assimilation rendu plus aisé par la correspondance entre les modes de vie auxquels conquérants et conquis étaient accoutumés. Au Soudan, tout au moins, il n'y eut aucune tentative de révolte chez les peuples conquis, et leur sort n'était en rien pénible. Très rapidement, ils participaient aux conquêtes des armées azande. Sans les obstacles créés par les Arabes, puis par les Européens, le processus de conquête, de colonisation et d'intégration, aurait pu se poursuivre jusqu'à ce que l'extension à certaines zones écologiques ait obligé les Azande à abandonner leur mode de vie traditionnel, car leur organisation politique dépassait celle de la plupart de leurs voisins. Nous remarquons aussi que les mélanges ethniques provoquèrent une différenciation de classe difficilement déterminable, entre les Ambomu et les Auro d'origine étrangère, qui se traduisait par des divergences de valeurs, d'habitudes et de langage. A ces égards, un Mbomu se considérait comme supérieur, spécialement du fait de ses nombreuses visites aux Cours princières. Cependant, la fréquence des migrations, la mobilité sociale et l'abondance des mariages mixtes, favorisèrent, un peu plus à chaque génération, la disparition de cette différenciation de classe. 
De toutes ces considérations, il s'ensuit qu'un des résultats de l'expansion vongarambomu est la dispersion des clans ambomu ainsi que des peuples sous leur joug. Il n'est peutêtre pas absolument vrai, comme les Azande aiment à le croire, qu'autrefois, les clans ambomu aient vécu en communautés plus ou moins séparées, chacune sur son propre territoire et sous l'autorité de ses propres anciens, et que c'est seulement à la prise du pouvoir par les Avongara que les clans se mélangèrent. Mais il est significatif qu'ils entretiennent cette idée, qu'ils considèrent que la dispersion des clans et, consécutivement, l'atrophie de leur leadership héréditaire, facilitèrent l'avènement de la prédominance politique des Avongara. Les clans sont certainement aujourd'hui très largement dispersés. Dans tout le pays zande du Soudan, on trouve des membres des principaux clans ambomu et de ceux de certains peuples assimilés; et, selon les renseignements recueillis sur les royaumes azande situés hors du territoire soudanais, ils seraient répartis à travers toute la région occupée par les Azande (Evans-Pritchard, 1959).

La présence d'un grand nombre de clans ne s'observe pas simplement au niveau de chaque royaume et de chaque province, mais dans chaque région. Dans une petite communauté typique de 263 adultes mâles (dont $30 \%$ d'Ambomu), 63 clans étaient représentés ; dans une autre, sur 54 adultes mâles (dont $17 \%$ d'Ambomu), on trouvait 26 clans représentés; enfin dans une troisième de 99 adultes mâles (dont $13 \%$ d'Ambomu), on comptait 37 clans. Les communautés locales azande ne sont donc aujourd'hui, en aucune manière, des groupes claniques. Bon nombre de personnes, dans chaque communauté, peuvent être unies par la parenté ou alliées par le mariage, mais, en tant que groupe, chaque communauté constitue une unité politique et administrative ayant devoir d'allégeance envers un roi ou un gouverneur de province, à travers son délégué résident. C'est ce qui donne à la communauté son unité et son originalité, et c'est seulement en ce sens qu'elle forme un groupe local (Evans-Pritchard, 1960 b). J'ajouterai que les Azande prétendent que les Ambomu sont plus largement représentés dans les provinces autrefois gouvernées par les rois, car ils aiment conserver leurs relations avec la monarchie, en tant que descendants des premiers affidés de la maison royale vongara. Il reste encore à déterminer le réalité de cette affirmation en analysant les chiffres du recensement.

Il est possible que la dispersion des clans explique, jusqu'à un certain point, l'importance de leur nombre. Les Azande disent que les clans furent dispersés, puis que leurs fragments le furent à nouveau, si bien qu'individus et groupes perdirent contact avec les autres membres de leurs clans, fondèrent de nouveaux groupes généalogiques, se rattachèrent aux clans ambomu ou à de vastes clans étrangers, de sorte qu'on ne sait plus qui appartient ou n'appartient pas à la souche mère. Au recensement, on dénombra 188 clans représentés chacun par au moins 25 adultes mâles inscrits, mais beaucoup d'autres offraient une moindre représentation. On n'en connaît pas encore le nombre, mais si l'on considère que l'enquête ne porta que sur une partie du pays zande du Soudan, que la preuve est faite de la présence en pays zande, hors du Soudan, d'éléments ethniques différents de ceux des royaumes soudanais, on peut conclure à l'existence de plusieurs centaines de clans. L'origine étrangère de beaucoup d'entre eux se manifeste dans leurs noms, dont les Azande essaient d'expliquer la signification, après assimilation aux sons de leur propre langue, des syllabes dont ils sont composés (Evans-Pritchard, 1956 b).

Le nombre des clans et leur dispersion posent des problèmes historiques confus et emmêlés qui se compliquent encore du fait que des personnes d'un même clan peuvent avoir 
des totems différents. Les Azande expliquent ces divergences par l'habitude, mentionnée plus haut, qu'avaient les individus et les groupes de se rattacher à des clans connus, parfois désignés sous le nom de kpamiakpamia, agrégat, clan. Peu importe l'explication; le fait est que les membres d'un même clan à l'intérieur d'une même communauté locale peuvent avoir des totems différents. J'ai dénombré 127 totems pour les Azande du Soudan : 57 mammifères, 22 oiseaux, 34 reptiles, 1 crustacé, 13 insectes (Evans Pritchard, 1956 c). Sur un échantillonnage de 42 clans, parmi les mieux connus, dans un cas seulement tous les membres du clan avaient le même totem. Parmi les gens qui prétendent appartenir à un même clan et ont des totems différents se rangent les Agiti et les Abakpuro. Sur 289 Agiti, 225 ont pour totem le serpent rungbu (les serpents sont les totems azande les plus communs, spécialement chez les Ambomu), 40 la «bête tonnerre » (les Azande considèrent le tonnerre comme une créature), 21 le léopard, 2 le serpent rungbara et un le serpent d'arbre. Sur 62 Abakpuro, 6 ont le serpent rungbu, un la «bête tonnerre », 5 le léopard, 48 un chat sauvage et 2 la genette. Ces données sont une preuve supplémentaire du peu d'importance de l'appartenance à un clan dans la société politiquement bien organisée des Azande (EvansPritchard, 1961).

Comme il fallait s'y attendre, la contrepartie de ce mélange ethnique est une culture composite, reconnue comme telle par les Azande. L'économie originale mbomu a dû subir de grandes modifications au cours des cent ou deux cents dernières années. La plupart des plantes vivrières des Azande : manioc, patates douces, maïs, arachides, tabac, toutes d'origine américaine, n'ont pu atteindre le pays zande avant le XVIe siècle, et probablement pas avant le XVIIe, le XVIIIe, et même le XIXe siècle, et uniquement par l'intermédiaire d'un autre peuple africain, puisqu'elles se répandirent avant la pénétration européenne. Les Azande ont également appris à cultiver la banane ainsi que leur ressource essentielle d'aujourd'hui l'éleusine, de même que le ficus (dont ils utilisent l'écorce intérieure pour la fabrication des pagnes) et leurs principales légumineuses. En tenant compte de ces indications et de quelques autres, on peut supposer qu'avant d'entreprendre leurs migrations, les Ambomu étaient d'abord chasseurs et secondairement agriculteurs, tandis qu'aujourd'hui les Azande sont surtout agriculteurs. Ce changement d'économie aide à comprendre leurs migrations et possède également une signification politique, car le développement politique qui se produisit certainement lors de l'expansion territoriale, l'accroissement du pouvoir, l'extension de l'organisation administrative chez les Avongara et l'assimilation des peuples étrangers sont peut-être liés à la plus grande stabilité qu'entraîna la dépendance à l'égard des récoltes (Evans-Pritchard, $1960 \mathrm{c}$ ),

Sous certains aspects, la culture zande est faite de pièces et de morceaux. Des articles ultérieurs montreront que leur art, leur artisanat, une grande partie de leurs techniques ainsi que certaines de leurs institutions importantes furent empruntés par les Ambomu aux peuples étrangers voisins ou assujettis ${ }^{1}$.

Le facteur de cohérence et de stabilité de ce mélange hétérogène d'éléments ethniques et culturels fut l'organisation politique supérieure des Avongara-Ambomu qui leur permit d'imposer leur langue et leurs institutions aux peuples conquis. J'examinerai maintenant cette organisation. Les Azande vivaient traditionnellement dans des habitations isolées, séparées les unes des autres par quelques centaines de mètres de brousse, parfois davantage. Les

Parmi ces articles, lire : A Further Contribution to the Study of Zande Culture, Africa, 1963. 
premiers voyageurs notèrent qu'à certains moments, les habitations disparaissaient complètement pendant plusieurs kilomètres, et ils se rendirent compte qu'ils passaient alors d'un royaume à un autre. Ces royaumes semblent avoir été organises sur le même modèle.

Durant l'expansion zande, plusieurs dynasties organisèrent des conquêtes, mais les membres des différentes maisons royales étaient tous de la même famille ou du même clan, c'est-à-dire des Avongara. Je laisse de côté le clan des Bandiya, d'origine ethnique très différente, qui règne sur les Azande de la région située à l'extrémité ouest du pays, ainsi que sur le peuple allié des Nzakara, encore plus à l'ouest. De Dampierre étudia cette région et rectifia mes erreurs dues à des sources antérieures. Je laisse également de côté un petit groupe d'Azande, les Adio ou Makaraka qui occupent l'extrémité nord-est du pays zande, et sont soumis, non pas à l'autorité des Avongara, mais à celles de leurs propres anciens. Les dynasties avongara descendent toutes du roi Ngura, chef du peuple mbomu, il y a sept ou huit générations, et furent fondées par ses fils et ses petits-fils sur les territoires qu'ils s'étaient partagés. Je crois que nous pouvons nous fier aux témoignages azande à ce sujet.

Comment les Avongara devinrent-ils les maîtres des Ambomu ? La question reste ouverte. On les a dit d'une race étrangère (non-mbomu) et d'intrus, mais aucune preuve ne vérifie cette thèse. Les faits connus ne confirment en rien l'opinion, d'ailleurs contraire à la logique, selon laquelle ils ont un langage secret. Une telle opinion peut ne provenir que de l'habitude des petits-fils des princes de parler le zande en inversant les syllabes (EvansPritchard, 1954). Les Azande ont leur propre version de l'origine de leur maison royale (Evans-Pritchard, 1957 a), et, en dépit de quelques invraisemblances, je la pense la plus plausible : un individu nommé, croit-on, Basenginonga domina un peuple divisé, grâce à sa sagesse et à son hospitalité. Ses descendants accrurent leur autorité et leur puissance, affirment les Azande, au cours de migrations et de guerres. De nombreux exemples de l'histoire africaine prouvent que bien des individus ont ainsi rassemblé des partisans et fondé des dynasties : tels Chaka, Sebotoane, Moshesh et d'autres. Plus proches des Avongara, nous avons le cas des Bandiya déjà mentionnés et celui des Mangbetu qui règnent sur un ensemble de peuples dont les institutions politiques ressemblent à celles de leurs voisins azande. Seul le dernier cas nous fournit la preuve certaine que l'initiative d'un seul homme (peut-être un Vongara) ait pu être à l'origine d'une dynastie régnante. L'arbre généalogique de Basenginonga à Ngura demeure incertain, en revanche, il y a tout lieu de considérer l'arbre généalogique de Ngura jusqu'à nos jours comme réellement exact.

Les descendants de Ngura fondèrent six dynasties dont les généalogies ont été soigneusement conservées par de nombreux observateurs, et de Calonne dressa la carte des territoires qu'ils gouvernaient. Des descendants de chaque dynastie se fixèrent comme souverains dans les royaumes indépendants dont le nombre variait de temps à autre, selon les vicissitudes des guerres. A aucun moment il n'est possible de préciser leur nombre, d'abord en raison du manque d'informations adéquates, mais aussi en raison de l'obligation, qui deviendra bientôt manifeste, de définir les éléments constitutifs d'un royaume en termes relatifs. Cependant, si nous définissons un royaume comme une région dont les habitants reconnaissent la souveraineté d'un seul homme, qui n'en reconnaît lui-même aucune, alors, vers 1880 , il y avait une quinzaine de royaumes. Leur étendue variait, mais leur traversée nécessitait toujours plusieurs jours de marche. Le royaume de Gbudwe s'étendait approximativement sur 25000 kilomètres carrés. Il nous faut deviner le chiffre de leur population, mais, d'après certaines indications, elle ne descendait vraisemblablement pas au- 
dessous de 50 000, et atteignait probablement le voisinage de 100000 dans les royaumes les plus vastes comme celui de Gbudwe.

Cependant, bien qu'un royaume ait des frontières nettement délimitées par les rivières, lorsque les Azande débattent des relations entre souverains et sujets, ils mettent l'accent sur l'allégeance personnelle plutôt que sur la tenure du sol. En un sens, il est certain que le roi possédait les terres et qu'il pouvait en expulser un sujet, que tout individu, vivant sur le domaine où il exerçait son pouvoir, devenait ipso facto son sujet. Mais personne n'était lié à une terre particulière, ni à une région. La densité de la population était faible - quatre habitants au kilomètre carré, moins peut-être - et les gens s'installaient où il leur plaisait et déplaçaient fréquemment leurs cases. La propriété foncière avait peu d'importance dans le contexte politique. Il n'était pas question de payer un fermage sous forme de tribut ou de prestations de travail. En outre, si un Zande n'était pas satisfait de son sort dans un royaume, il s'en allait dans une autre. Cette migration devait sans doute s'opérer avec discrétion, néanmoins il partait et jurait allégeance au souverain du royaume où il allait désormais vivre. L'identification des habitants d'un royaume par référence à une personne et non à un territoire illustre bien la considération des relations en terme d'allégeance personnelle plutôt que de propriété, de souveraineté plutôt que de possession. Ainsi les habitants d'un royaume étaient avuru Wando, sujets ou partisans de Wando, ceux d'un autre royaume étaient avuru Malingindo, sujets ou partisans de Malingindo, etc. On peut comparer cette désignation de la communauté politique à celle utilisée en Europe avant que l'idée de possession ne la supplante, mais tandis qu'en Europe elle se définissait par référence à un peuple -roi des Francs, due des Normands, roi des Anglais - chez les Azande, elle se définissait en référence au souverain. Lorsqu'ils parlent d'un royaume, les Azande ne pensent pas tant à ses frontières qu'aux relations d'un ensemble de sujets avec une personne. Les rois se souciaient plus de gagner des partisans que des terres, durant leur lutte pour le pouvoir; l'acquisition de nouveaux territoires résultait de celle de nouveaux sujets. Nous verrons qu'en tant que territoire, un royaume ne durait pas longtemps. A la mort de son roi, il se subdivisait en royaumes séparés sous l'autorité de ses fils aînés, et les habitants de chaque nouveau royaume devenaient les sujets de souverains indépendants, alors que ces souverains avaient reconnu la suprématie de leur père. En conséquence, on ne peut comparer un royaume zande au royaume de Bunyoro, par exemple, où l'on a une succession de monarques, dont l'autorité peut être clairement définie en termes territoriaux, tout comme la succession des dynasties en Angleterre.

Pour comprendre le mode de désignation des communautés politiques chez les Azande, ainsi que la conception qu'ils s'en faisaient, peut-être faut-il s'en rapporter à leur passé, au temps de leurs grandes migrations durant lesquelles des populations entières se déplaçaient, comme nos ancêtres les Germains. Le lien entre les chefs de ces hordes migrantes et leurs partisans ne pouvait être alors que personnel, et non pas fonction d'un territoire quelconque. La manière de s'adresser au souverain nous laisse aussi penser que la maison royale vongara n'a pas toujours joui du prestige que notèrent les premiers voyageurs européens, mais qu'elle l'a acquis au cours de ses migrations et des guerres qui les accompagnaient, comme les Azande eux-mêmes le disent et comme d'autres sources nous le confirment : "La guerre fait le roi. » Ce n'est que par rapport à l'histoire, au passé, que les Azande mettent l'accent sur l'aspect territorial du royaume. Ils se réfèrent alors aux possessions du roi, kumbo Ezo, le royaume (ou l'héritage) d'Ezo. 
Avant de décrire l'organisation structurelle d'un royaume zande, il convient de préciser le sens des termes employés, en particulier la traduction du mot zande gbia. Ce mot peut être utilisé pour désigner une personne de rang supérieur, dans un rapport d'autorité et de subordination qui, pour les Azande, se retrouve dans toutes les relations sociales, mais au sens strict il ne s'applique qu'aux membres du clan des nobles avongara. Il nous faut cependant opérer une distinction entre les divers statuts des membres de la noblesse. Le roi gouvernait un royaume du type défini ci-dessus. Je ne parle de «princes » que dans un sens politique : ces membres de l'aristocratie régnaient sur la province d'un royaume, représentant le monarque qui les nommait et devant qui ils étaient responsables. Par le mot «nobles », je désigne tous les membres du clan aristocratique, et j'attribue à ce mot un sens social plutôt que politique, car bien que roi et princes aient aussi été des nobles, la plupart des membres du clan aristocratique n'ont joué récemment aucun rôle politique, sinon mineur, tout en jouissant d'une position sociale supérieure accompagnée de certains privilèges. Je désigne par commoner governor le citoyen ordinaire (commoner), nommé par le roi à la tête d'une province, pour opérer une distinction entre les gouverneurs issus du peuple et les gouverneurs issus de la noblesse, les princes. Les Azande emploient souvent le mot «Azande » pour différencier les citoyens ordinaires des nobles, mais je ne l'utiliserai que dans un sens culturel plus vaste, pour désigner le peuple tout entier : l'aristocratie vongara, les descendants de leurs premiers partisans ambomu et les descendants des peuples conquis, les Auro.

Le roi divisait son royaume en provinces délimitées par des rivières. Il s'en réservait une grande au centre. Dans chacune des autres, il nommait un représentant qui gouvernait en son nom. Sa position centrale lui permettait de ne pas être trop éloigné de la Cour de ses gouverneurs, mais elle lui permettait aussi d'éviter les attaques surprises d'un autre royaume. Les attaquants auraient dû franchir une ou plusieurs provinces-frontières, laissant ainsi à tout le royaume le temps d'être alerté. De larges sentiers conduisaient de la Cour royale aux Cours provinciales, et les gouverneurs devaient veiller à ce que les herbes trop hautes soient coupées au ras du sol de chaque côté. Junker présente la gravure d'un homme accomplissant cette besogne. Au début du règne d'un roi, les gouverneurs étaient souvent des citoyens ordinaires, car, en général, le roi se méfiait de ses parents. Mais lorsque ses fils grandissaient - même parfois dès l'adolescence - il leur confiait ses principales provinces. Chaque gouverneur exerçait, dans sa province, la même autorité que le roi dans la sienne; les fonctions des gouverneurs ne différaient de celles du roi que par leur extension; leurs Cours construites sur le modèle de la Cour royale fonctionnaient de la même façon. Cependant, un roi pouvait destituer un gouverneur selon son bon vouloir s'il le soupçonnait de déloyauté, de lâcheté ou de mauvaise gestion, et il pouvait, à son gré, le transférer d'une province à une autre. Aux environs de 1900, la structure administrative du royaume de Gbudwe, que j'ai analysée en détail (Evans-Pritchard, 1960 d), semble avoir compté vingt-six provinces, la sienne y compris, gouvernées en majeure partie par ses fils. De temps à autre, le nombre des provinces d'un royaume variait et le roi pouvait en modifier les frontières s'il le jugeait bon. Leurs dimensions se modifiaient aussi, et dans une certaine mesure, leur degré d'autonomie. Celle que gouvernait le second fils de Gbudwe, Mange, à l'est de la rivière Sueh, était si vaste, si peuplée et si éloignée de la Cour, qu'il régnait presque en souverain indépendant, et, bien qu'il reconnût formellement l'autorité de son père, il s'en tenait à l'écart.

L'aménagement administratif de chaque province se calquait dans l'ensemble sur celui du royaume. Chaque province constituait un royaume en miniature. Le prince dans sa province était comme le roi dans son royaume. Il installait sa Cour au centre pour la protéger des raids 
et la rendre accessible à ses délégués. Ces délégués, ou députés, correspondaient aux gouverneurs des provinces du royaume, mais jouissaient d'un pouvoir et d'une autorité moindres. Ils étaient chargés des différents districts qui, pour des raisons administratives, composaient la province, et ils faisaient respecter les ordres du gouverneur. Ils étaient responsables devant lui du bon ordre, du paiement du tribut en nature et en travail, du règlement des querelles qui ne nécessitaient pas l'intervention du gouverneur lui-même, du service militaire, etc. De même que des sentiers conduisaient de la Cour royale aux Cours provinciales, d'autres sentiers plus étroits menaient de la Cour provinciale aux habitations des délégués du gouverneur de province.

La question de succession ne se posait pratiquement pas dans ces provinces ou fiefs. J'emploie ce mot sous toutes réserves car les provinces ressemblaient peu aux fiefs de la féodalité européenne. Lorsqu'un prince mourait, le roi le remplaçait, soit par un jeune frère utérin du défunt, soit par quelqu'un d'autre, à son gré. Il envoyait un homme de la Cour présenter le nouveau prince à ses sujets qui l'acceptaient, à titre officiel, après quelques discours pour lui enjoindre de gouverner sagement. A cet égard, les sujets n'avaient pas le choix. Un prince pouvait, s'il le voulait, charger un de ses jeunes frères ou de ses fils aînés, responsable devant lui, d'exercer l'autorité sur une partie de son territoire, sans que le roi s'y oppose. Celui-ci considérait le fief comme un tout et ne traitait qu'avec le prince qui en avait la responsabilité. Un prince ne pouvait aliéner son fief. A la mort du roi, lorsqu'une province était gouvernée par un noble et non pas par un citoyen ordinaire, le fief devenait de facto royaume indépendant et son prince devenait roi, s'il survivait aux luttes meurtrières qui survenaient alors.

Il faut souligner que si l'autorité royale était reconnue dans tout le royaume, que si les gouverneurs, nobles ou citoyens ordinaires, payaient soigneusement le tribut au roi, lui rendaient visite chaque mois ou à peu près (Mange était une exception) et évitaient de lui déplaire, il n'en reste pas moins que le roi n'exerçait son pouvoir que sur une partie restreinte de ce royaume ; pour le reste du territoire, il devait s'en remettre à d'autres. Son droit effectif sur ses sujets passait donc aux mains de ses gouverneurs, et en particulier de ses fils aînés, qui eux-mêmes en déléguaient une partie à leurs représentants. Étant donné les dimensions d'un royaume zande et l'état rudimentaire du réseau de communications, on imagine qu'il y avait une limite à l'étendue du territoire et à la population sur lesquels un homme pouvait exercer personnellement et directement son contrôle.

Le système de provinces peut être considéré comme une structure segmentaire complexe, au sein de laquelle naissaient certaines oppositions. Celles-ci s'exprimaient sous forme de jalousie et d'inimitié, en particulier entre les princes des provinces voisines. Des combats s'ensuivaient parfois, se limitant habituellement à des échauffourées sans importance, car le roi les considérait tous comme ses sujets et punissait le coupable. En dépit des changements de gouverneurs, un particularisme local tendait à subsister, car les gens maintenaient des liens avec celui qui avait été leur seigneur à un moment donné. Les gens de la province du fils de Gbudwe, Rikita, n'étaient pas seulement avuru Gbudwe, sujets de Gbudwe, mais aussi avuru Rikita, sujets de Rikita, distincts des sujets des autres princes. C'était avec Rikita et non avec son père qu'ils avaient des contacts personnels, c'était par rapport à lui qu'ils concevaient leurs devoirs. Cependant, des rivalités se développaient entre les princes, mais non pas entre les princes et le roi, car celui-ci était trop puissant pour qu'aucun de ses fils ne le renversât. Il avait à sa disposition immédiate une force efficace, et pouvait compter sur 
l'appui de ses autres gouverneurs, trop jaloux les uns des autres pour s'allier contre lui. Donc, s'il est vrai que, à l'instar de ce qui se passait dans l'Europe féodale, les liens unissant un sujet à son chef immédiat étaient plus forts, parce que plus personnels, que ceux le rattachant à son roi lointain, personne n'avait cependant à choisir entre deux allégeances.

Dans chaque royaume zande pour lequel nous bénéficions d'informations suffisantes, le même processus se répétait. Le roi établissait ses fils dans les provinces. Même de son vivant, ses fils aînés, installés depuis longtemps dans les plus vastes provinces, jouissaient d'une indépendance presque totale et régnaient en leur propre nom, dans des États autonomes. A la mort du roi, ils acquéraient leur autonomie complète et s'employaient à agrandir leur patrimoine aux dépens des peuples voisins et de leurs frères plus faibles. Lorsqu'ils avaient conquis leur propre royaume, ils établissaient leurs fils et ainsi de suite, de génération en génération. Aucun doute ne subsiste sur de tels faits consignes à plusieurs reprises, notamment par de Calonne.

On peut imaginer que durant la période de pleine expansion, il y a de cela quatre ou cinq générations, les fils aînés, qui, à la mort de leur père, héritaient d'un vaste patrimoine, agrandissaient leur domaine aux dépens des peuples étrangers plutôt que de s'épuiser en guerres civiles dans le but de dominer. Lorsque l'expansion fut enrayée, parce que limitée dans la plupart des directions, et lorsque chaque territoire de la maison royale eut acquis une certaine stabilité, les princes ne disposèrent plus que d'une seule possibilité d'agrandir leur domaine : conquérir ceux de leurs frères ou de leurs autres parents. La guerre civile et l'élimination de la plupart des princes offraient la seule alternative à une fragmentation sans fin.

Considérons maintenant les rapports entre gouvernants et gouvernés, entre le roi ou son représentant et ses sujets, et la manière dont on les envisageait de part et d'autre. Des deux côtés, on estimait que découlaient du mythe même de l'origine du clan vongara les principales fonctions royales : fonctions judiciaires, militaires, économiques ou fiscales, de même qu'administratives. Comme chaque gouverneur avait dans sa province les mêmes fonctions que le roi dans la sienne, ce que je dis du roi vaut simultanément pour ses gouverneurs, à moins que je ne précise le contraire.

Toutes les affaires d'une certaine importance, les crimes et préjudices qui nécessitaient un châtiment ou une compensation, se réglaient à la Cour royale, habituellement devant le roi lui-même qui prononçait les jugements et infligeait des sanctions en fonction de la coutume et des cas antérieurs. Il quittait ses appartements personnels pour rendre la justice tous les deux ou trois jours. Les hommes de la Cour, assis en demi-cercle devant lui, l'assistaient. La plupart des affaires azande touchaient à la sorcellerie ou à l'adultère. Comme les preuves péremptoires faisaient habituellement défaut, il fallait déterminer les faits en interrogeant l'oracle (Evans-Pritchard, 1937). Tandis que chacun pouvait consulter son propre oracle dans ses propres affaires, seul l'oracle du roi ou d'un de ses représentants accrédités avait valeur en matière de justice. L'organisation de la justice reposait donc entièrement entre les mains du roi et son premier devoir était de s'assurer qu'elle fonctionnait bien, et qu'aux yeux des Azande, les jugements rendus étaient équitables et raisonnables. En tant que seul maître de la justice, le roi recevait des honoraires et des dons des parties gagnantes. 
En plus de son rôle de juge suprême, le roi assurait aussi le commandement en chef des forces de son royaume. Les compagnies de guerriers, divisées en compagnies d'adultes et en compagnies de jeunes gens, possédaient chacune leurs propres officiers. Les compagnies de jeunes gens avaient leurs casernes près de la Cour. Quelques-uns d'entre eux y résidaient généralement. Ces soldats de carrière, pour ainsi dire, étaient des volontaires. Le prince n'avait aucun mal à les recruter. Entre autres incitations, il leur offrait la perspective de faciliter leur mariage ; or, trouver une épouse dans une société où la plupart des femmes se mariaient aux nobles ou aux citoyens les plus riches et les plus âgés, posait souvent un problème. Lors d'un raid à travers un royaume voisin ou d'une guerre entre deux royaumes, tous les effectifs d'une province ou d'un royaume pouvaient être mobilisés et incorporés dans l'armée pour la durée des combats. Les hommes de la province attaquée devaient se rendre immédiatement au lieu de bataille et n'offraient donc pas la résistance d'une force organisée. Des raids frontaliers (basapu) menés par les gouverneurs des provinces limitrophes se produisaient tous les ans ici ou là. Lors de l'attaque d'une province, les provinces voisines venaient à son secours, mais ces raids n'occasionnaient pas une mobilisation générale du royaume. Les échauffourées de cette espèce ne duraient que quelques heures, et ne semblent guère avoir eu d'autre but que de relever le prestige d'un prince et de prouver la loyauté de ses sujets. Cependant leur fonction sociologique était le maintien d'un système politique dans des royaumes aux forces égales : l'état latent d'agitation menaçante faisait de la frontière une limite politique et accentuait les allégeances respectives à tel ou tel roi et à ses représentants (Evans-Pritchard, 1957 b). Les guerres ou les campagnes de grande envergure entre royaumes, ou contre les Arabes et les Européens (sungusungu wura), qui pouvaient se prolonger plusieurs jours et même plusieurs semaines, étaient assez rares. Durant les trentesix ans que dura son règne, Gbudwe n'en mena que neuf. Lors de ces luttes, le roi envoyait des messagers à ses gouverneurs de provinces avec ordre de mobiliser leurs forces et de se rendre, avec leurs compagnies, sur le lieu des combats, si le royaume se trouvait en danger et s'il s'agissait d'une opération défensive. Seul le roi pouvait ordonner la mobilisation générale du royaume, et il semble que, d'ordinaire, il dirigeait personnellement la stratégie et les tactiques de la campagne, avec l'aide de son oracle, encore que chaque contingent ait été sous les ordres directs du gouverneur de sa province d'origine et qu'il ait eu à résoudre ses propres problèmes d'intendance (Evans-Pritchard, $1957 \mathrm{c}$ ). L'organisation militaire du royaume reposait donc entre les mains et du roi et de ses représentants; c'est surtout en temps de guerre que les habitants d'un royaume réalisaient leur appartenance à une unité distincte, à une communauté politique sous l'égide d'un roi. La participation des hommes d'un royaume à la guerre prouvait leur dépendance vis-à-vis d'un roi et non d'un autre, et c'était la seule activité qui les regroupait tous. L'action militaire collective d'un royaume zande constituait le plus sûr critère de délimitation de ce royaume.

Le roi se considérait comme responsable, au sens large, de l'ordre publie, du maintien des communications et de la transmission des nouvelles sur l'étendue de son royaume. Il n'intervenait pas dans les affaires intérieures des gouverneurs de provinces, à moins que leurs rivalités ne les conduisent à des échauffourées ou que soit flagrante l'incapacité de l'un d'eux. Mais il ne supportait pas qu'ils prennent des libertés avec lui, et il s'attendait à être averti, par messagers, en cas d'événements fâcheux ou d'attaque éventuelle du royaume, ainsi qu'à être tenu au courant de la situation dans les provinces, tous les mois ou tous les deux mois, par les gouverneurs en personne. Il insistait particulièrement sur le devoir des gouverneurs de provinces limitrophes, chargés de surveiller les frontières, de s'informer à l'avance, par leurs espions et leurs oracles, de l'imminence d'une attaque, et sur leur devoir de se rendre 
immédiatement au secours de la province menacée, le cas échéant. Le roi, dans sa province centrale, de loin la plus vaste, assumait, à travers ses délégués, des fonctions administratives équivalentes à celles des gouverneurs dans les leurs.

Le maintien d'une cour de justice, d'une organisation militaire et administrative impliquait la présence de nombreuses personnes à la Cour : les fils du roi, ses parents, les gouverneurs en visite, les délégués qui présentaient leurs hommages, les jeunes soldats qui résidaient dans les casernes, les plaignants qui attendaient leur procès, les pages, les suppliants et les autres, qu'il fallait nourrir pendant leur séjour qui pouvait se prolonger audelà de plusieurs jours, voire de plusieurs semaines dans les provinces où les gouverneurs n'avaient pas toujours, au moment précis, les moyens d'aider les solliciteurs qui ne seraient pas restés s'ils avaient obtenu satisfaction immédiate. Leur présence nécessitait une quantité de nourriture que le roi ne pouvait acquérir que par le travail et les dons de ses sujets. Il avait trois manières de se procurer la nourriture qu'il offrait à ses hôtes. Les compagnies militaires travaillaient dans les plantations royales. Dans sa province, les compagnies et les femmes des guerriers adultes défrichaient la brousse pour préparer les récoltes royales, les sarclaient et les moissonnaient. Des détachements, envoyés par chacun des commoner governors du royaume, les aidaient dans les tâches les plus pénibles, notamment dans le défrichement. Il ne faut pas confondre la culture de ces jardins par un travail public et celle des jardins privés du roi. Ses femmes et leurs servantes, aidées de ses pages personnels, s'occupaient de ces derniers. En théorie, comme en pratique probablement, les récoltes cultivées par les sujets servaient, dans l'ensemble, à nourrir les étrangers et non les propres habitants de la maison royale, mais il est probable qu'en cas de besoin on puisait à l'une ou l'autre source. Le second apport de nourriture provenait du tribut, sorte de taxe que payaient au roi les sujets de sa propre province. Mais, bien qu'une certaine pression ait pu être exercée sur les personnes qui ne venaient pas régulièrement à la Cour pour les contraindre à verser leur contribution, les Azande la considéraient plutôt comme une sorte de souscription qui, de temps à autre, permettait d'approvisionner leur souverain en biens dont ils bénéficiaient lors de leur séjour à sa Cour. Les délégués de chaque district recueillaient auprès des gens les produits de saison provenant des jardins ou de la brousse, et les envoyaient à la Cour. Les gens offraient aussi une partie du gibier qu'ils tuaient. Encore une fois, ce tribut n'était pas destiné au roi et à ses femmes, mais servait à entretenir l'hospitalité à la Cour. Si un sujet souhaitait contribuer à agrémenter le menu personnel du roi avec un mets particulièrement savoureux, du gibier par exemple, ou de la bière, il lui en faisait don personnellement et de façon privée. La troisième source de biens en nature, dont seul le souverain suprême bénéficiait, était le tribut annuel que lui envoyaient les gouverneurs de provinces. Chaque année, des caravanes apportaient au palais royal une partie du tribut versé aux gouverneurs de provinces par leurs sujets. La préparation de la nourriture se faisait sous la responsabilité du roi. Elle incombait à ses femmes et à leurs servantes qui devaient, par conséquent, être fort nombreuses - j'en reparlerai plus loin.

Outre les revenus alimentaires que lui fournissaient gouverneurs et sujets, le roi recevait encore, pour sa propre maison, de nombreux objets d'art fabriqués par les artisans : paniers, chapeaux, nattes, pagnes de fibres, tabourets, jattes, marmites, etc. Dans le cas du royaume de Gbudwe, certains produits, rares dans sa province, abondaient dans d'autres et étaient donc doublement bienvenus. On lui offrait encore des lances, des objets de métal ouvragé et d'autres articles de valeur comme prix d'amendes, comme honoraires de la cour de justice, comme butin de guerre, etc. Certains de ces objets lui servaient à acquérir de nouvelles 
épouses, mais il devait en distribuer la plupart à ses guerriers et aux suppliants qui imploraient son aide, non seulement dans sa province, mais aussi dans celles des gouverneurs. Les prisonniers de guerre, garçons et filles, lui étaient remis, mais il en redistribuait un certain nombre ; de même, il faisait don d'épouses à ses courtisans et à ceux qui avaient servi dans ses compagnies militaires. Ces femmes, en dehors des captives de guerre, semblaient avoir été offertes au roi en guise d'amende ou arrachées aux hommes qui avaient encouru la disgrâce royale. J'ignore si les princes offraient souvent des épouses à leurs sujets pour les récompenser, mais je crois le fait assez rare ; pourtant les Azande et leurs princes considéraient ce geste comme l'exemple suprême de la munificence royale.

Dans une économie de subsistance, sans monnaie ni marché, un souverain n'avait guère d'autre solution que de distribuer le surplus de nourriture et d'objets tels que chapeaux, pagnes de fibres, etc. Quoi qu'il ait pu échanger les lances contre des femmes, la plupart du temps, il les donnait à ses sujets, car un roi ou un prince ne pouvait repousser un solliciteur, même s'il le faisait attendre longtemps. Ce que nous venons de décrire constitue, certes, une forme d'échange de dons, mais, pour sa compréhension exacte, une telle notion requiert des considérations supplémentaires. Le sujet offrait des présents à son souverain, celui-ci les redistribuait; mais la signification psychologique du don diffère dans chacun des cas. Le sujet remplit son devoir et le souverain accepte gracieusement le tribut offert. Il fait une faveur au sujet en acceptant son don, puis en lui distribuant ses largesses : dans les deux cas, le sujet est en état d'infériorité, et, bien qu'il s'agisse d'échanges, ceux-ci se situent sur un plan différent. La plupart des donateurs ne recevaient rien en retour, car ils ne fréquentaient pas les cours. La nourriture et les autres dons étaient partagés entre les serviteurs de la Cour : le corps de garde, les parents du roi, les pages, les délégués, les gouverneurs et leur suite, etc. Dans l'ensemble, le peuple assurait la subsistance de la Cour. Il restait cependant un surplus au roi et aux princes. Il ne faut pas oublier non plus que grâce à cette distribution de nourriture et de cadeaux, les souverains pouvaient maintenir une organisation politique qui leur permettait d'accumuler une fortune par les amendes, les honoraires, les présents destinés à s'assurer les privilèges, etc. Ils étaient les membres les plus riches de la société, comme le prouvaient leurs vêtements (simples, mais de meilleure qualité que ceux des citoyens ordinaires), leur air de bien nourri, et plus particulièrement leur abstention des travaux manuels et le nombre de leurs femmes qui dépassait, et de beaucoup parfois, celui que pouvaient atteindre les citoyens les plus fortunés. Dans une économie telle, les femmes constituaient la vraie richesse par leur travail et par les enfants qu'elles mettaient au monde, par leurs fonctions de production et de reproduction. Plus un homme avait de femmes, plus il pouvait offrir l'hospitalité, plus il était entouré de personnes qui travaillaient pour lui et le servaient, et plus il avait d'enfants. Outre l'avantage économique que leur procurait le nombre important de leurs épouses, les souverains exploitaient aussi leurs sujets qui devaient travailler pour eux et leur verser un tribut. Si les services assurant, grâce aux dons de tous, le maintien de l'État, bénéficiaient à tous, il est vrai que tous n'en profitaient pas dans la même mesure. La strate supérieure de la société, les Avongara, recevait des avantages plus substantiels que les citoyens ordinaires. Cependant, ce serait une erreur de raisonner en termes purement économiques. Les rois ne désiraient pas tant la richesse que le prestige personnel ; ne pourrait-on même soutenir que les princes aimaient les biens dans la mesure où le don qu'ils en faisaient leur assurait un renom ? Ce renom importait au premier chef et, dans l'esprit du vulgaire, les avantages économiques constituaient la rétribution du pouvoir qu'il s'employait à soutenir. 
Il nous faut noter ici que la fonction royale zande était purement profane, dénuée de toute attribution religieuse et magique. Le pouvoir royal se suffisait à lui-même. Les relations entre souverain et sujets étaient d'ordre pratique, et naturels les services mutuellement rendus. Les Azande se considéraient comme les enfants-sujets d'un roi qu'ils estimaient être le père et le souverain du peuple. En lui payant un tribut, ils lui exprimaient leur respect, et lui, en retour, offrait l'hospitalité à quiconque lui rendait visite. Lorsqu'on s'entretient avec eux, les citoyens azande s'avouent souvent batailleurs et indisciplinés, et ils admettent que, sans leurs nobles maîtres, le désordre régnerait, car ils refuseraient d'obéir à l'un de leurs pairs, ce que je pense exact. Ils reconnaissent le bien-fondé d'une autorité supérieure dont nul ne peut contester les décisions, et c'est pourquoi ils lui obéissent. Ils acceptaient autrefois qu'en dernier ressort le roi utilisât la force si ses décisions étaient mises en question, ses ordres non exécutés, et si quelqu'un agissait à l'encontre des lois, des coutumes, des bonnes manières. Le roi avait la force à sa disposition avec ses compagnies militaires, sa suite, ses délégués et en général les membres de sa Cour. Il leur ordonnait d'exécuter ou de mutiler un individu, de saisir ses femmes, de détruire sa maison, et personne ne contestait son droit d'agir de la sorte. Cependant, il existait un frein au despotisme. Si un prince essayait de trop exiger de ses sujets, poursuivait leurs femmes, se montrait cruel, ceux-ci avaient la possibilité de réagir. Ils pouvaient cesser de lui rendre visite, l'isolant par là même. S'ils sentaient que ni leur personne, ni leurs biens n'étaient plus en sécurité, ils s'en allaient dans une autre province et juraient allégeance à son gouverneur. Un prince impopulaire s'apercevait aussi qu'au cours des guerres civiles qui suivaient la mort de son père, ses sujets lui refusaient leur aide. Mais il existait un autre élément modérateur peut-être plus efficace encore que la crainte de perdre des sujets ou de se voir refuser leur aide. Les royaumes azande se classaient dans la catégorie de Montesquieu où régnait le principe de l'honneur. Rois et princes se sentaient obligés de se conduire selon les traditions attachées à leur statut, d'être courtois envers leurs sujets, de ne pas dépasser la limite prescrite par la coutume et d'agir avec dignité. L'un dans l'autre, si le pouvoir royal semblait illimité, du moins en théorie, en pratique le souverain le déléguait à des représentants, qui n'avaient de possibilité, d'action que dans la mesure où ils conservaient la confiance de ceux auprès de qui ils étaient envoyés. Les premiers voyageurs notèrent l'abîme qui séparait l'apparence de la réalité. En dernier ressort, le roi ne pouvait obtenir que ses sujets lui obéissent que pour autant que ceux-ci aient décidé de le faire.

A mon sens, le secret du succès de l'organisation administrative zande résidait dans la délégation de l'autorité. A tous les niveaux, le roi ou le prince gouvernait par ses représentants ; gouverneurs, délégués, chefs des compagnies militaires. Sans eux, il était impuissant. Il quittait rarement sa maison et passait la majeure partie de son temps avec ses femmes et ses jeunes enfants. Être non seulement lointain, mais aussi solitaire, il n'aurait eu aucune idée de ce qui se passait, encore moins aurait-il pu le contrôler, sans le loyalisme de ceux qu'il nommait aux diverses charges. Ces hommes transmettaient à d'autres l'autorité du roi, si bien qu'en y participant, beaucoup de personnes accroissaient leur propre prestige. En définitive, l'autorité du roi était fonction de sa répartition du haut en bas de l'échelle. En un mot, les délégués surtout exerçaient l'autorité royale et en jouissaient. Ils pouvaient parfois la circonvenir et l'utiliser au mieux de leurs intérêts et de leur prestige. Bien des choses s'accomplissaient au nom du roi, qu'il n'avait jamais commandées et dont il n'entendrait peutêtre jamais parler. On exécutait des gens en son nom, mais non sur son ordre. Nous voulons dire par là que sous l'apparent despotisme du souverain se cachait toute une bureaucratie qui servait la puissance royale pour le privilège d'y participer, par simple plaisir personnel, par amour du pouvoir, même d'un pouvoir délégué, et qui réussissait grâce à ses relations 
particulières avec le souverain et à des visites fréquentes à sa jour. Je dirai maintenant quelques mots des aménagements de la Cour (Evans-Pritchard, 1957 d).

Le roi vivait au milieu de son royaume comme la reine des abeilles au milieu de sa ruche. Sa Cour se divisait en trois parties : la cour extérieure, la cour intérieure, les appartements privés ou harem. Dans la cour extérieure, on réglait les problèmes judiciaires, on décidait les guerres; le roi en personne ou, en son absence, un de ses fils, traitait les questions administratives ; quotidiennement on y envoyait la nourriture pour le personnel de service, les membres des compagnies, les délégués, et pour tous ceux que l'ambition amenait régulièrement à la Cour ; quant aux citoyens ordinaires, à moins qu'ils n'aient eu quelque problème spécial à y résoudre, ils s'en tenaient à l'écart, car si la Cour pouvait attirer des récompenses : emploi, don, renommée, elle Présentait aussi des risques : on pouvait être accusé de magie, de sorcellerie ou d'adultère avec les femmes du roi et on courait le danger d'être ensorcelé par des rivaux jaloux des faveurs royales. La plupart des gens, spécialement les étrangers, préféraient rester chez eux et s'occuper de leurs affaires. Le cas échéant, ils portaient leurs ennuis devant le délégué du roi dans leur district. Une petite cour intérieure appelée «le lieu des secrets », et près de laquelle étaient construites les cases des pages du roi, séparait la cour extérieure des appartements privés. Les nobles qui mangeaient entre eux et les fonctionnaires les plus âgés y recevaient leur nourriture. Un page les mandait depuis la cour extérieure lorsqu'on avait apporté les jattes et les marmites des appartements privés. Le roi pouvait aussi s'y entretenir, loin de la foule, avec les hauts dignitaires de sa suite. Un sentier reliait cette cour au harem royal qui occupait une vaste superficie, en raison du nombre important d'épouses, et du fait que chacune d'elles avait sa propre case et son propre jardin; dans le voisinage se trouvaient aussi les lieux de culture. Les femmes devaient cultiver ce qu'il leur fallait pour elles et leurs enfants, puis préparer leur nourriture ainsi que celle des sujets des deux cours royales. Le roi nommait cuisinière l'une d'entre elles, qui seule préparait ses repas. A la Cour du roi ou l'un riche prince, les femmes étaient organisées en compagnies, semblables aux compagnies militaires, avec chacune à sa tête une matrone que nous appellerons reine. Les sujets entretenaient les cultures royales publiques dont ils offraient le produit, ainsi que leur tribut en nature, à l'une ou l'autre reine qui les redistribuait à ses suivantes chargées de préparer la nourriture pour les cours. Le maître donnait aux reines, et parfois aux autres femmes, des servantes, habituellement des jeunes filles, qui effectuaient les besognes les plus pénibles. Tôt ou tard, le roi avait des rapports avec elles secrets, dit-on, par crainte des ragots du harem - et, si elles lui donnaient des enfants, il leur accordait une case et elles acquéraient le statut d'épouse.

Une des caractéristiques les plus importantes de la Cour royale zande, et qu'on ne retrouve pas dans les monarchies avec lesquelles l'histoire nous a familiarisés, est la dimension des familles engendrées par les nombreuses épouses. Certains rois avaient des douzaines de femmes. En fait, un souverain pouvait demander la main de n'importe quelle jeune fille célibataire bien qu'il dût payer une dot ; quoi qu'il en soit, les gens étaient heureux de compter leurs filles parmi les épouses royales à cause du prestige et des avantages qu'ils pouvaient en tirer. Le roi ne traitait pas cependant sa belle-famille avec le respect qu'on attendait d'un citoyen ordinaire. Les relations entre roi et sujet n'étaient pas modifiées par la relation beau-fils - beau-père. Comme le roi avait des rapports conjugaux avec toutes ses femmes, on peut facilement imaginer pourquoi le clan royal est maintenant le plus important probablement des clans azande. Au recensement effectué en territoire soudanais, il était le plus nombreux avec 1382 mâles, adultes ou presque. Si sa représentation dans les autres 
parties du pays zande est du même ordre, le clan des Avongara compte probablement plus de 10000 personnes de tous âges. En admettant qu'elles descendent toutes du roi Ngura qui vivait il y a sept ou huit générations, le nombre est étonnant mais non pas improbable. On peut le comparer à l'accroissement des familles royales des peuples Shilluk et Anuak, ou à celui de la famille Sanusi en Cyrénaïque où la loi coranique limitait la polygamie. Cet accroissement du clan royal des Azande contribua à la formation d'une caste ou classe aristocratique, non pas à une simple différenciation entre souverains et sujets, mais à l'émergence d'une strate privilégiée qui, de par sa naissance, jouit d'un statut supérieur, bien que la plupart de ses membres ne détiennent aucune charge. Ils évitent autant que possible le travail manuel, mais la plupart ne peuvent s'y soustraire totalement, et, à chaque génération, la situation devient pour eux plus difficile. Leur accroissement entraine une diminution proportionnelle de leur prestige et de leur richesse, et, à part quelques-uns, ils sont de plus en plus obligés de vivre comme des citoyens ordinaires. Aussi nous faut-il distinguer aujourd'hui entre les princes et la classe aristocratique, noble par naissance, mais sans autorité politique. Je parle de ce qui se passait il y a trente ans, lors de mon séjour chez les Azande. La situation fut différente dans le passé. Au fur et à mesure que nous reculons dans le temps, le nombre des Avongara diminue. Certaines indications nous laissent supposer qu'ils vivaient moins largement lorsqu'ils étaient moins puissants. Jadis, en outre, les Azande agrandissaient leurs possessions et les fils de la famille royale pouvaient être dotés d'une province, si bien que leur densité était plus faible et que les termes «gouvernant» et «noble » étaient synonymes. Il faut aussi se rappeler que nombre d'entre eux périrent à la guerre ou furent assassinés. Nous reviendrons bientôt sur ce point.

Les fils des princes avaient une vie familiale plus courte et moins intime que les fils des citoyens ordinaires. Ils restaient avec leur mère jusqu'à l'approche de la puberté. Le roi ou leurs frères aînés leur enjoignaient alors de quitter le harem et de construire leur case à proximité de la cour extérieure. A partir de ce moment, ils n'entraient plus jamais au harem. Ils ne voyaient leur père que lorsqu'il venait dans la cour extérieure, et ne voyaient leur mère qu'occasionnellement, lorsqu'ils s'arrangeaient pour la rencontrer à la limite du domaine royal. Si leur père les gâtait dans leur jeune âge, il leur fallait supporter par la suite ce qu'aucun fils de citoyen ordinaire n'endurait. Ils avaient souvent faim. Leurs frères aînés se servaient d'eux pour leurs corvées, et leur administraient une correction s'ils n'accomplissaient pas le travail rapidement et convenablement. Cette éducation les habituait certainement à l'indépendance et de surcroît leur formait le caractère. La plupart des Européens qui rencontrèrent les rois et les princes azande au temps de leur puissance leur ont rendu hommage. Rusés et impitoyables, les souverains avongara, s'ils voulaient survivre, devaient ressembler aux princes italiens du temps de Machiavel. Mais ce que les Européens admiraient en eux, c'était la dignité naturelle de leur maintien, leur fierté sobre, leurs manières courtoises, leur cordialité, leur sang-froid, leur réserve, leur intelligence, leur prudence, etc. (Evans-Pritchard, 1957 e). La fierté et la réserve, la timidité même, l'attitude distante en certains cas, les manières raffinées, la simplicité des atours, la perspicacité de jugement, le degré supérieur d'intelligence, la pondération et l'air d'autorité sont autant de caractéristiques que j'ai rencontrées chez presque tous les princes, et même chez les nobles sans responsabilités politiques. Au premier coup d'œil, on pouvait les distinguer des citoyens ordinaires.

Les fils aînés recevaient de leur père, alors qu'ils n'étaient encore parfois que des adolescents, le gouvernement des provinces du royaume. S'ils étaient très jeunes, un 
courtisan en qui le roi avait confiance les guidait. Tôt ou tard, le roi dotait tous ses fils d'un fief, à moins qu'il ne les jugeât incompétents. Une mutuelle réserve présidait aux relations entre ces princes et leur père. L'étiquette de la Cour interdisait toute intimité entre eux et imposait une certaine retenue. Les pères soupçonnaient toujours leurs fils aînés d'essayer de séduire leurs épouses, mais ces soupçons reposaient davantage sur les révélations des oracles que sur des faits réels. Bien que les cas de rébellion des princes contre leurs pères aient été fort rares, ceux-ci craignaient toujours d'être les victimes de la déloyauté de leurs fils ou d'un assassinat comploté par leurs sorciers. Je reparlerai des relations entre frères après quelques considérations sur les filles des rois.

Il n'est pas douteux que les Avongara, surtout les hauts dignitaires, n'approuvaient pas, dans l'ensemble, le mariage de leurs filles avec des citoyens ordinaires, même si parfois ils les leur accordaient. Les hommes ne sollicitaient pas de telles unions qui semblent avoir été, en général, instables et peu satisfaisantes. Les maris n'exerçaient qu'un faible contrôle sur leurs épouses de sang royal, qui n'étaient pas préparées par leur éducation oisive à mener la vie rude et active d'une femme du peuple. Les maris n'appréciaient pas particulièrement les rapports incestueux que les nobles avaient régulièrement avec toutes leurs parentes, à l'exception de leurs sœurs germaines, car ils savaient que si un homme rendait visite à sa demi-sœur ou à sa cousine, il mettrait peut-être à profit son séjour pour avoir des relations avec elle. De plus, le statut social des fils issus de ces unions mixtes présentait quelque ambiguïté : nobles et gens du peuple avaient tendance à trouver qu'ils se donnaient des airs. En conséquence, bien qu'il soit difficile de le savoir exactement, il semble que les jeunes filles de noble naissance aient été données en mariage à des parents, parfois pour nouer une amitié, mais que, le plus souvent, leur père ou leurs frères les aient gardées comme maîtresses, car les nobles n'hésitaient pas à avoir des relations sexuelles avec leurs propres filles ou leurs propres sœurs par lignée paternelle. Les autres princesses devenaient plus ou moins courtisanes, comme les princesses Shilluk, passant, suivant leur fantaisie, d'un homme à l'autre, noble ou roturier, et elles finissaient probablement dans le harem de quelque prince, mais sans que nous sachions si c'était à titre de cousine ou d'amante ou les deux à la fois. On leur donnait alors des servantes et elles menaient une vie facile. Les Azande ajoutent que beaucoup s'adonnaient à des pratiques lesbiennes. Comme la femme vivait en état de soumission dans la société zande, sa participation aux affaires politiques, impliquant une autorité sur les hommes, aurait été considérée comme inconvenante.

Revenons aux fils des rois. Il convient de penser les lois de succession aux fonctions royales en termes de réalités historiques plutôt qu'en termes de principes, car il ne semble y avoir eu ni droit d'aînesse, ni aucun ordre de succession. Et même, à proprement parler, il n'y avait pas de succession. Bien sûr, les fils aînés bénéficiaient d'une position plus avantageuse que les jeunes fils pour hériter de leur père à sa mort, puisqu'ils étaient déjà fermement établis comme gouverneurs de principautés presque autonomes. Lorsque, dans le passé, il leur était loisible d'étendre leur domination aux dépens des peuples étrangers, ils se souciaient peu d'essayer d'annexer les provinces de leurs pairs, mais la compétition devenait plus âpre lorsqu'il s'agissait d'acquérir le domaine royal, avec son prestige et sa population nombreuse. Des combats réglaient le différend, chaque prétendant recevait le soutien de sa province ; on m'a dit qu'en fait le prince qui jouissait de la confiance des sujets de son défunt père avait toutes chances de l'emporter, leur soutien se révélant décisif. Ainsi Gbudwe, qui, à la mort de son père, se trouva dépourvu de province, au moins d'importance, obtint cependant la charge du domaine paternel, parce qu'il fut choisi par les guerriers du roi défunt. On 
comprendra facilement que c'était là, pour un prince, une puissante incitation à se comporter correctement avec ses sujets. Les gouverneurs de provinces ne pouvaient compter que sur le loyalisme à leur égard. Leurs sujets ne leur étaient attachés, ni par allégeance traditionnelle à une lignée généalogique locale, ni par fidélité à un clan prédominant dans la région, ni par un lien utérin du prince avec sa principauté, ni par quelque autre manière observable dans les sociétés africaines. Pour cette même raison, les princes ne s'opposaient pas au roi.

Les rivalités dynastiques, tout au moins au cours des périodes plus récentes de l'histoire zande, occasionnèrent des guerres et des crimes fréquents auxquels les Arabes et les Européens ajoutèrent leur part. Les faits prouvent, avec certitude, que l'histoire zande forme une longue suite de parricides, de fratricides, de meurtres de fils et de cousins, comme l'histoire visigothe (EvansPritchard, 1958 b). Effroyable massacre! Très peu de rois mouraient de mort naturelle et même ceux qui, à notre avis, mouraient de la sorte, périssaient, au regard des Azande, pour qui toute mort est provoquée intentionnellement, sous l'effet des malédictions ou de la magie de leurs parents. Seuls les plus rusés et les plus impitoyables survivaient à ces luttes. Nous pouvons reprendre la citation de Ganshof à propos des querelles entre fils et petit-fils de Clovis, au vie siècle : «ils ressemblaient à des bêtes sauvages qui s'entredéchiraient »(Ganshof, 1952). Ces rivalités, principalement entre frères, surgissaient du fait qu'à la mort du roi aucun fils n'héritait de son domaine. Chacun devenait monarque dans son ancienne province. Les plus faibles trouvaient plus commode de rendre hommage aux plus forts. Les plus forts luttaient pour la possession du domaine personnel du roi défunt. Lorsque ce différend était résolu, ils se battaient, parce que, comme je l'ai précédemment noté, c'était lors des guerres que les sujets prouvaient leur loyauté au souverain, mais les Azande interprétaient différemment l'action en disant que les rois faisaient la guerre par fierté, pour l'honneur et la renommée. La situation ressemblait fort à celle de la royauté franque, pour la raison suivante : dans les deux cas, pendant la vie du roi aussi bien qu'à sa mort, le royaume était divisé entre ses fils. La prévision des luttes de domination et de survie qui auraient invariablement lieu à la mort de leur père ne pouvait pas ne pas affecter l'attitude des princes azande les uns envers les autres, et nous ne serons pas surpris de ce que rivalité, suspicion et haine aient été les sentiments des frères royaux les uns vis-à-vis des autres. On comprendra aussi que, dans ces circonstances, la rébellion d'un prince contre son père eût été folie, car aucun d'entre eux n'était assez puissant pour triompher seul, et leurs rivalités personnelles prévenaient toute alliance efficace susceptible de renverser la monarchie.

J'ai essayé de brosser un vaste tableau du système politique zande, dans la mesure où la reconstitution est possible, en insistant plus spécialement sur la place de la royauté, et j'ai mis en évidence certains aspects qui me semblent significatifs, si l'on veut comprendre les institutions politiques de ce peuple en particulier, et des États africains en général. Je n'ai donné que peu de détails, car ils sont contenus dans les ouvrages et articles cités dans la bibliographie qui suit. Je ne pense pas qu'une recherche plus approfondie puisse aujourd'hui fournir beaucoup d'autres éléments que ceux que j'ai apportés sur les sujets traités. Ce sont, comme disent les Azande, de vieilles choses, des choses du passé (kuru pai). Je me demande s'il vit encore un seul Zande qui se souvienne avoir vu le roi Gbudwe. Cependant, une recherche complémentaire nous conduirait à noter les changements des institutions au cours d'un demi-siècle de domination étrangère, et contribuerait à les éclairer rétrospectivement. 


\section{BIBLIOGRAPHIE du chapitre 5}

ANTINORI (0.), 1868. Viaggi di 0. Antinori e C. Piaggia nell' Africa Centrale, Boll. Soc. Geog. Ital., 1, pp. 91-165.

CALONNE-BEAUFAICT (A. de), 1921. Azande, Bruxelles.

CASATI (Gaetano), 1891. Ten years in Equatoria and the return with Emin Pasha, trad. anglaise, 2 vol., Londres et New York.

CZEKANOWSKI (Jan), 1924. Wissenschaftliche Ergebnisse der deutschen Zentral-AfrikaExpedition 1907-1908, unter Führung Adolf Friedrichs Herzog zu Mecklenburg, Bd 6, 2 : Forschungen in Nil-Kongo-Zwischengebiet, Leipzig.

EVANS-PRITCHARD (E. E.), 1937. Witchcraft, Oracles and Magie among the Azande, Oxford.

- $\quad$ 1954. A Zande Slang Language, Man, 1954, 289.

- $\quad$ 1955, 1956, 1957. Zande Historical Texts, Sudan Notes and Records, 36, pp. 123145 ; 37, pp. 20-47 ; 38, pp. 74-99. (Others are about to appear in Kush and elsewhere.)

- $\quad 1956 a$. A history of the kingdom of Gbudwe, Zaire, 10, t. 1, pp. 451-491

t. II., pp. 675-710, 815-860.

1956 b. Zande Clan Names, Man, 1956, 62.

1956 c. Zande Totems, Man, 1956, 110.

$1957 a$. The origin of the ruling clan of the Azande, Southwest. J. Anthrop., 13, pp. 322-343.

1957 b. Zande Border Raids, Africa, 27, pp. 217-231.

1957 c. Zande Warfare, Anthropos, 52, pp. 239-262.

1957 d. The Zande royal court, Zaire, t. I, pp. 361-389, 493-511 ; t. II, pp. 687713.

1957 e. Zande kings and princes, Anthrop. Quart., 30, pp. 61-90.

$1958 a$. The ethnic composition of the Azande of Central Africa, Anthrop. Quart., 31, pp. 95-118.

1958 b. An historical introduction to a study of Zande Society, Afr. Stud., 17, pp. $1-15$.

1959. The distribution of Zande clans in the Sudan, Man, 1959, 24. - 1960 a. Zande Cannibalism, J. R. Anthrop. Inst., 90, pp. 238-258. 
$1960 \mathrm{~b}$. Zande clans and Settlements, Man, 1960, 213.

$1960 \mathrm{c}$. A contribution to the study of Zande culture, Africa, 30, pp. 309-324.

$1960 \mathrm{~d}$. The organization of a Zande kingdom, Cahiers d'Études Africaines, 4, pp. 5-37.

1960 e. The ethnic origin of Zande office-holders, Man, 1960, 141.

1961. Zande clans and totems, Man, 1961, 147.

GANSHOF (F. L.), 1952. Feudalism, trad. anglaise, Londres, p. 3.

HUTEREAU (A.), 1909. Notes sur la vie familiale et juridique de quelques populations du Congo belge, Ann. Mas. du Congo Belge, sér. 3.

- 1922. Histoire des peuplades de l'Uele et de l'Ubangi, Bruxelles.

JUNKER (Wilhelm), 1890. Travels in Central Africa during the years 1875-1878, trad. anglaise, Londres.

1891. Travels in Central Africa during the years 1879-1883, trad. anglaise, Londres.

- 1892. Travels in Central Africa during the years 1882-1886, trad. anglaise, Londres.

LAGAE (C. R.), 1926. Les Azande ou Niam-Niam, Bruxelles.

LARKEN (P. M.), 1926. An account of the Zande, Sudan Notes and Records, 9, pp. 1-55.

1927. Impressions of the Azande, Sudan Notes and Records, 10, pp. 85-134. PIAGGIA (Carlo), 1941. Le memorie di Carlo Piaggia a cura di G. Alfonso Pellegrinetti, Florence.

SCHWEINFURTH (Georg), 1873. The Heart of Africa, trad. anglaise, Londres, 2 vol.

VANDEN PLAS (V._H.), 1921. La langue des Azande, vol. 1 : Introduction historicogéographique, Ghent. 


\section{Chapitre 6 \\ "Le cannibalisme zande" * (1960)}

\section{$\underline{\text { Retour à la table des matières }}$}

Malgré la difficulté de reconstituer avec quelque certitude le passé d'un peuple primitif, à partir de ses traditions et de ses souvenirs, de telles sources, rassemblées par un enquêteur compétent, présentent, dans la plupart des cas, une valeur supérieure aux récits des premiers Européens. Ces récits, lorsque les problèmes analysés réclament plus qu'une simple observation et d'élémentaires conclusions, doivent être examinés avec soin et utilisés avec précaution, notamment lorsqu'il s'agit d'un sujet aussi extraordinairement attirant que le cannibalisme des sauvages, qui semble avoir été d'un intérêt morbide pour les Européens. Je ne dis pas que les voyageurs mentirent délibérément sur ce qu'ils avaient vu, ou inventèrent ce qu'ils n'avaient pas vu, mais il est certain qu'ils émirent souvent des affirmations sans les prouver, qu'ils témoignèrent d'un réel manque de jugement dans l'interprétation des faits et qu'ils embellirent leurs descriptions de suppositions et d'exagérations, même lorsqu'ils avaient reçu une formation scientifique.

Le problème qui se pose est le suivant: les Azande d'Afrique centrale étaient-ils cannibales ? Nombre de voyageurs visitèrent le pays zande avant la domination européenne et même avant la tentative malheureuse du gouvernement égyptien d'y imposer ses lois. On pourrait donc croire facile de résoudre le problème, car ces voyageurs venaient du nord en

\footnotetext{
Paru dans le J.R.A.I., 1960.
} 
compagnie d'Arabes et de Nubiens, tous convaincus que les Niam-Niam - alias les Azande étaient cannibales. Ainsi prévenus, ils étaient à l'affût de preuves confirmant leurs suppositions. Cependant, lorsque nous examinons, comparons et jugeons leurs déclarations, nous nous mettons à douter de la validité de leurs témoignages et de leurs interprétations. Le problème n'est donc pas aussi simple que je l'ai exprimé, car il faut tout d'abord savoir ce qu'on entend par «Azande », par « cannibalisme », et dans quelle mesure on le pratiquait le cas échéant.

Le commerçant gallois, John Petherick, fut le premier Européen à entrer en contact avec les Azande. Nous ignorons de quels Azande exactement il s'agissait, mais probablement d'immigrants installés à la périphérie nord-est de la zone d'expansion zande et très mélangés d'éléments étrangers. Il rapporte les propos des Baer (Mberidi), vivant au nord du pays zande, et décrivant leurs voisins du sud «comme des guerriers et des sauvages qui immanquablement se régalaient de leurs victimes ennemies » (1861, pp. 455-456). On l'informa aussi (1861, pp. 468-469) que si un esclave échappé de chez son maître zande était repris, on le tuait et on le mangeait. Il ajoute encore qu'il «sut par les Neam-Nam, qui apparemment s'enorgueillissaient de leur réputation de cannibales, que les vieillards et tous ceux que l'on supposait proches de la mort étaient tués et mangés ». La connaissance qu'avait Petherick des Azande était négligeable, et sur le sujet en question, ses propos relèvent de simples ouïdire. Sa femme qui l'accompagna lors de son dernier voyage répète avec lui dans Travels in Central Africa que les Azande étaient cannibales (1869, 1, p. 280). Au sujet des Abarambo, confondus désormais avec les vrais Azande qui les ont complètement assimilés, politiquement, socialement et culturellement, ils écrivent : «Dès qu'un individu expire, on échange son corps contre des lances, au plus offrant : pour un cadavre en bon état, on en donne entre quinze et vingt. L'acheteur, après avoir mis de côté ce qu'il lui faut pour sa consommation personnelle, vend le reste au détail comme un boucher le fait avec un mouton» $(1869,1$, p. 275). Petherick n'alla jamais chez les Abarambo; là encore, il ne peut donc s'agir que de ouï-dire.

L'Italien Carlo Piaggia, chasseur et marchand, fut le premier Européen à pénétrer profondément en pays zande. Il y résida de 1863 à 1865, principalement à la Cour du roi Tombo. Ses aventures furent collationnées, d'après ses renseignements, par son ami le marquis 0. Antinori, également chasseur et commerçant. Antinori montre très clairement (1868, p. 124) que, bien que Piaggia ait vécu plusieurs mois seul dans l'intimité des Azande, il n'avait rencontré aucun indice de ce qu'on appelle habituellement cannibalisme, si ce n'est un cas isolé de vengeance: «Più accanite poi sono le zuffe che avvengono con tribù straniere ; in allora spingono la rete di vendetta fino a divorarsi le carni degli uccisi, ed il Piaggia in una di queste guerre ne fu testimonio : d'onde par sia venuta lorola trista celebrità di cannibali. » Néanmoins, dans son Memorie, paru en 1941 (Passamonti l'avait utilisé auparavant dans sa biographie de Piaggia), il note (p. 290) : "Gli uomini in armi si riuniscono nei boschi e vengono presto a soffrire la fame, perchè la selvaggina, atterrita dal rumore delle armi, fugge lontano. Spesso stanno di fronte, senza attaccar battaglia, anche dieci e quindici giorni. Cos! quando vengono alle mani sono pieni di odio e di fame, e si affrettano, cessato il combattimento, a tagliar le carni migliori ai caduti, per arrostirle e mangiarle. » Il ajoute encore (1941, p. 196) que lors d'une partie de chasse avec de jeunes garçons, ils atteignirent un coin de brousse où les sujets du roi Tombo et ceux de son frère, le roi Bazingbi, s'étaient battus quelques semaines plus tôt. Le sol était jonché de cadavres en putréfaction et il remarqua, avec étonnement, qu'à la plupart d'entre eux il manquait certaines 
parties du corps. Il entendit ses compagnons dire qu'on les avait mangées. «Domandai chi avesse mangiato le carni che vedevo tagliate con ferro, ed essi risposero: Gli uomini di Tombo. » Il rapporte encore un épisode désagréable dans lequel le roi était impliqué (pp. 234-235). Tombo invita Piaggia à manger. Comme il n'arrivait pas à identifier la viande qu'on lui présentait, il en aurait pris aussi peu que les convenances le lui permettaient, si Tombo ne l'avait pressé d'en manger davantage. Le repas achevé, il partit se promener en brousse. Il s'entendit interpeller et aperçut dix sauvages immobiles, appuyés sur leurs lances. Jetant un nouveau regard, il vit un homme pendu à la branche d'un arbre, les pieds traînant à terre. "Giratogli attorno vidi due grandi tagli nelle natiche, dalle quali era stata asportata gran parte della carne. Ritornato verso i selvaggi, che apparivano melanconici, domandai che cosa fosse accaduto. Tombo - risposero - ha sorpreso quest'uomo con una sua donna proprio in questo punto. Lo ha ucciso con un colpo di lancia, Io ha attaccato all'albero e tagiliata una parte delle sue carni Io ha lasciato cos!. Infatti io avevo scorto una larga ferita sul petto che non aveva dato una goccia di sangue. » Il lui vint alors à l'idée que c'était des fesses humaines que le roi Tombo lui avait fait manger. Il retourna en hâte pour demander une explication, ce qui lui valut la réponse déconcertante : "Il mio popolo è anche il tuo e se tu non fai vedere che Io mangi quand' è cattivo, non rispetterà le tue donne. È cos! che fanno tutti i grandi. » Cependant, dans ce qui semble être une autre version de la même histoire, les détails diffèrent tellement qu'on en arrive à douter de la véracité de son témoignage (1941, p. 236). L'uomo est devenu un giovinetto che poteva contare i diece anni, exécuté pour un délit autre que dans le premier cas, et Tombo et quelques-unes de ses femmes se partagent le repas. Piaggia ne put assurer que Tombo et ses femmes mangeaient la chair du jeune garçon, et il crut préférable de ne pas s'en informer. "Poichè anche riflettevo alle di loro parole in dei primi giorni che restai solo fra loro ebbi più volte da inquietarmi verso loro, che mi ridevano dicendo che il bianco era cattivo per mangiare troppo sale, ma le carni dovevano essere buone. E cosi ogni qualvolta avevo da inquietarmi, mi sentivo dire : il bianco ha mangiato molto sale. » (Nous ignorons exactement en quelle langue Piaggia s'entretenait avec ses hôtes.) La seule indication que nous ayons nous laisse supposer que, même à la fin de son séjour chez les Azande, il ne parlait qu'imparfaitement leur langue, si bien que je suis sceptique lorsqu'il déclare qu'il surprit des conversations. Il ne pouvait évidemment comprendre les Azande au cours de ses premières journées parmi eux, aussi nous faut-il admettre, ou bien qu'il était accompagné de quelques personnes qui parlaient arabes ou bien qu'il laissait aller son imagination. L'éditeur de Piaggia, Pellegrinetti, insinue (1941, pp. 7-21) que le Memorie fut écrit de mémoire et que l'auteur a pu faire quelques concessions au sensationnel, sur le conseil de ses amis.

Cependant, ni les écrits de Petherick, ni ceux d'Antinori ne sont à l'origine de la réputation de cannibalisme des Azande. Depuis le Moyen Age, chez les Arabes d'Égypte et du Soudan, la tradition s'est transmise, de l'existence, en Afrique centrale, d'un peuple cannibale désigné, avec quelques variations, sous le nom de Niam-Niam. Les récits des Arabes firent leur apparition dans la littérature européenne à la fin du XVIIIe siècle, dans les oeuvres de W. G. Browne (1799), de C.-L. du Couret (1854) (peut-être est-ce un faux, une de ces petites plaisanteries à la Alexandre Dumas ; mais même si l'auteur a pu broder, je crois le document authentique) et du baron Henri Aucapitaine (1857). Ils demeuraient trop vagues et trop fantaisistes pour permettre aucune précision sur les faits et sur leur origine. Lors de la pénétration dans la région de Bahr-el-Ghazal par les voyageurs et les commerçants, chacun ajouta sa quote-part au mythe. Ainsi le Savoyard Jules Poncet, dont la compagnie commerça avec les Azande de l'est à la fin des années 1850 et au début des années 1860, et bien qu'il ne 
les ait jamais vus lui-même, écrivait, en s'appuyant sur le témoignage de ses employés : «L'anthropophagie des Niam-Niam ne saurait être contestée désormais. Les gens de nos expéditions parmi lesquels se trouvaient des Européens ont été témoins de trop de faits pour qu'il y ait encore le moindre doute à cet égard. Ils enterrent leurs morts et ne mangent que les ennemis qu'ils tuent à la guerre. Leurs femmes les suivent dans leurs chasses et leurs expéditions contre leurs voisins, et ramassent, lors du carnage, les pieds et les mains des morts qui sont leurs morceaux de préférence » (pp. 38-39). De même, le naturaliste allemand Theodor von Heuglin, qui n'avait jamais visité personnellement le pays zande mais qui l'avait seulement traversé au nord en 1863, note (p. 206) que pour les marchands arabes du Soudan, le mot «Niamanian» signifie «mangeurs», spécialement «mangeurs d'hommes». Ces marchands déclaraient aussi que les Azande avaient des crocs, des têtes de chiens et des queues.

Cependant, quelles que soient les croyances qui avaient cours au Soudan, l'opinion scientifique aurait peut-être hésité à accepter les simples ouï-dire rapportés par des commerçants et des chasseurs, si Georg Schweinfurth, éminent botaniste russo-allemand de grande renommée, n'avait ajouté son témoignage aux leurs. En 1870, Schweinfurth voyagea pendant trois mois, avec une grande caravane arabe, dans les régions les plus à l'est du pays zande. Il commença par des allusions au cannibalisme (1873, vol. 1, p. 317) : un jour, six femmes esclaves brûlèrent dans une case ; «tandis qu'ils aidaient à nettoyer les débris, les esclaves niam-niam, récemment capturés, ne déguisèrent pas leur plaisir en sentant l'odeur de chair brûlée ». Plus loin, il note $(1873,1$, p. 449) qu'au centre de la concession zande se trouve un poteau «sur lequel on suspend les trophées de chasse. Sont fixés à ce pieu les crânes d'espèces peu communes, de splendides cornes d'antilopes et de buffles, auxquels s'ajoutent des crânes humains, des mains et des pieds desséchés 1 ». Ces allusions devinrent bientôt affirmations. Les sujets du roi Wando étaient cannibales. «Les preuves des habitudes de cannibalisme que nos regards étonnés rencontraient étaient trop évidentes pour être récusées. Près des cases, parmi les tas d'ordures, on voyait des os humains qui portaient la marque indiscutable des coups de hache ou de couteau ; et tout autour, sur les branches des arbres, pendaient des mains et des Pieds humains à demi réduits à l'état de squelette. Cependant, ils n'étaient ni assez secs, ni suffisamment cachés par les feuilles, si bien qu'une épouvantable et intolérable puanteur emplissait l'atmosphère» (1873, 1, p. 517). Il admet qu'il pouvait y avoir des exceptions, qu'il avait entendu parler de voyageurs, dont Piaggia, qui, au cours de leur passage en pays zande, plus à l'ouest (dans les domaines des rois Tombo et Bazingbi), n'avaient rencontré aucun signe de cannibalisme, et de certains chefs, comme Wando, qui repoussaient violemment l'idée de manger de la chair humaine. Cependant, il écrit (1873, II, p. 18) : "Tout bien considéré, ce que j'ai entendu et ce que j'ai vu, il ne fait aucun doute, à mon avis, que les Niam-Niam sont anthropophages ; ils ne cachent pas leurs appétits sauvages, portent ostensiblement au cou les dents de leurs victimes, ornent de crânes humains les poteaux dressés près de leurs habitations pour y exposer leurs trophées. On vend partout de la graisse humaine qui, absorbée en trop grande quantité, empoisonne, dit-on. En temps de guerre, on mange des gens de tous âges, mais plus particulièrement les vieillards qui sont de faciles et impuissantes proies face à la rapacité du conquérant. Si un individu solitaire et isolé meurt, sans que ses parents s'en préoccupent, il est sûr d'être dévoré dans le pays même où il vit. En bref, tous ceux qui seraient chez nous livrés au couteau de l'anatomiste, sont ici promis à ce destin mélancolique. » Les Azande ne niaient pas leur cannibalisme et bien que certains d'entre eux aient été si dégoûtés à l'idée de manger de la chair humaine qu'ils refusaient de partager le repas d'un cannibale $(1873,11$, p. 18), la 
plupart étaient cannibales $(1873,1$, p. 510). Il cite encore une autre preuve : quelques jours après un raid effectué chez les Babukur par les Azande et les Nubiens réunis, des indigènes, probablement des Azande, lui apportèrent des crânes fraîchement bouillis (déposés plus tard au Musée d'Anatomie de Berlin). Ils avaient entendu dire qu'il donnait des anneaux de cuivre en échange des crânes. C'est aussi quelques jours après ce raid que Schweinfurth - alors de passage au voisinage du mont Bangenzi - se trouva, au cours d'une de ses randonnées, dans une concession où une vieille femme, quelques jeunes garçons et filles, coupaient des calebasses en vue du repas, tandis qu'un homme jouait de la mandoline. Scène idyllique ? Que non 1 «Au milieu de l'espace qui séparait les deux cases, exposé au plein soleil de midi, sur une natte, un nouveau-né gisait, râlant ; je doute qu'il ait eu plus d'un jour. On me dit que c'était l'enfant d'une esclave capturée lors du dernier raid. La mère que l'on avait emmenée plus loin avait dû abandonner son bébé afin de pouvoir accomplir ses tâches domestiques sans autre préoccupation. La malheureuse petite créature condamnée à une vie si transitoire allait constituer un plat délicieux, et le groupe des sauvages vaquait à ses occupations habituelles jusqu'à ce que le pauvre petit être ait rendu l'âme et soit plongé dans le chaudron bouillant » (1873, Il, p. 223). Schweinfurth pensa d'abord intervenir, puis, par prudence, se ravisa.

La réputation légitime de Schweinfurth comme botaniste n'implique pas que nous devions nous fier également à ses observations ethnographiques. Par exemple, il note (1873, 1, pp. 90-91), à tort, que les ares et les flèches étaient les armes principales des Nuer. En fait, ils ne s'en servirent Jamais. Bien qu'il passât nombre de jours au milieu des camps d'éleveurs dinka, il ne remarqua pas une des caractéristiques frappantes de leurs troupeaux : la forme artificiellement imposée aux cornes des taureaux. Un dernier exemple, parmi d'autres : il signala (1873, 1, p. 18) que chez les Mangbetu, dont il fut l'hôte du roi, les seules mutilations corporelles pratiquées étaient le percement des oreilles et la circoncision. Il n'avait pas noté, aussi incroyable que cela puisse paraître, la déformation artificielle des crânes. Le pire est que, par endroits, il semble avoir pillé Piaggia sans le mentionner. Lorsqu'il écrit que les rois azande simulaient parfois des accès de colère et coupaient la gorge de leurs sujets de leurs propres mains (1873, Il, pp. 21-22), il est probable qu'il ait emprunté le fait au récit de Piaggia-Antinori (p. 112). Si l'on compare les deux auteurs, il ne fait aucun doute que Schweinfurth ait copié Piaggia (p. 125), lorsqu'il note par exemple (1873, 11, pp. 27-28) que les Azande ne versaient pas de dot lors du mariage, que si un homme désirait se marier il s'en remettait au roi ou à l'un de ses représentants pour lui trouver une femme, que lors de la cérémonie nuptiale, le souverain, accompagné de musiciens, de chanteurs et d'amuseurs, conduisait la mariée, en cortège, jusqu'au domicile du mari, et qu'un festin avait lieu ensuite. Il est faux, entre autres, que les Azande ne versaient pas de dot. Il est inutile de discuter l'origine des erreurs de Piaggia, mais de peur qu'on n'argue d'un changement de coutume entre le séjour de Schweinfurth et le mien, je dois faire remarquer que, quelques années seulement après Schweinfurth, Junker visita le pays zande et signala le paiement de la dot chez les Azande (1890, p. $344 ; 1892$, p. 261). Il ne faut pas oublier non plus que Schweinfurth ne resta chez les Azande que quelques semaines, que dans les circonstances troublées d'alors, il n'avait que peu d'occasions d'observer les mœurs, qu'il voyageait avec une grande caravane d'Arabes, accompagnés de leurs esclaves et de leur suite, qui devaient éviter tout contact Personnel avec la population, qu'il ne parlait pas zande et que les gens qu'il prit pour des Azande n'en étaient peut-être pas. Je reviendrai plus tard sur ce point. Ajoutons qu'en décembre 1870 , ses carnets contenant apparemment tout ce qu'il avait noté jusqu'à cette date, y compris sur les pratiques cannibales, brûlèrent, aussi dut-il se fier à sa mémoire; il est 
difficile de savoir s'il récrivit immédiatement, ou plus tard, ce qui avait disparu dans ce désastre.

Nous n'avons donc pas à nous incliner devant l'autorité de Schweinfurth à ce sujet. Lorsque nous examinons les preuves qu'il cite à l'appui de son affirmation selon laquelle la majorité des Azande étaient cannibales, il nous faut d'abord mentionner que sa connaissance de ce peuple était médiocre, limitée et superficielle (comment peut-il parler de la majorité des Azande ?) et qu'ensuite ce qu'il dit relève la plupart du temps de la pure conjecture et du simple ouï-dire. Il répète probablement ce que lui racontaient ses amis arabes et nubiens. Quant à l'histoire du bébé, elle sonne faux. Même si nous mettons de côté la question de la présence de ce bébé plusieurs jours après le raid, cette histoire va à l'encontre de tout ce que nous savons sur la façon dont les Azande traitent les enfants et qui nous interdit de croire qu'ils l'auraient laissé mourir s'ils avaient pu le sauver. Mais en dehors de ces considérations, Schweinfurth ne donne aucune preuve attestant que le bébé fut plongé dans la marmite puis mangé, ou qu'on avait même l'intention de le faire cuire et de le manger. En fait, cet exemple ne fournit aucune preuve concluante du cannibalisme des Azande. Je n'oublie pas les crânes, les mains, les pieds, les colliers de dents humaines et la graisse humaine vendue dans tout le pays. Il faut bien admettre qu'il vit au moins quelquefois des crânes humains, des pieds et des mains pendus à des poteaux et aux branches des arbres. Mais cela ne prouve pas que leurs possesseurs avaient été mangés, car les Azande rapportaient parfois de semblables trophées de la guerre. «Les crânes frais bouillis des Babukur » ne signifie rien, à mon avis, car on savait que Schweinfurth payait pour obtenir les crânes et on peut imaginer qu'il voulait des crânes et non des têtes. Quant à la vente de graisse humaine, je n'y crois pas. D'après son récit, il apparaît que Schweinfurth n'avait qu'une idée vague de ce qui se passait autour de lui, et je vois pas comment, même si l'on vendait de la graisse humaine, il aurait pu le découvrir. En outre, il ne dit ni en avoir vu, ni en avoir vu vendre. Il faut cependant rendre son dû au témoignage de Schweinfurth; les récits des autres témoins nous éclaireront sur sa valeur et nous permettront de le juger.

Mais s'il est quelqu'un, parmi les explorateurs du pays zande, dont le nom peut faire autorité, c'est Wilhelm Junker, docteur en médecine russo-allemand, qui passa, après 1875, plus de dix ans à explorer l'Afrique centrale, principalement en pays zande. Il parle peu de cannibalisme, si ce n'est pour remarquer qu'il était encore plus répandu chez les Mangbetu que chez les Azande, que les victimes de guerre étaient mangées (1892, p. 161), et que cette pratique valut aux Azande le surnom de «Niam-Niam », et aux Adio (Azande de l'Est) celui de «Makaraka », qui tous les deux signifient mangeurs d'hommes (1891, pp. 233-234). En y réfléchissant bien, il semble bizarre qu'un bon observateur comme Junker, qui séjourna parmi les Azande presque autant d'années que Schweinfurth avait séjourné de semaines, fasse à peine mention du cannibalisme, et qu'il n'avance aucune preuve pour appuyer ses assertions à ce sujet. Apparemment Junker, qui traversa le pays zande en tous sens, ne vit ni crânes, ni pieds, ni mains, ni graisse humaine, ni nouveau-nés destinés à la marmite. Son silence est d'autant plus significatif qu'il n'est pas le seul à n'en rien dire. Le colonel Chaillé Long, un Américain ignare, de caractère plutôt désagréable, au service du gouvernement égyptien, se rendit en 1875, avec l'explorateur australien Ernst Marno chez les Azande Adio (Makaraka) qui s'étaient établis à l'extrémité est du pays zande. Il déclare, comme tout le monde, que les Niam-Niam étaient cannibales (pp. 273-274), mais il n'en cite qu'une seule preuve (p. 287) lorsqu'il permit à ses compagnons azande d'attaquer les Nyangwara (qu'il appelle Yanbari), il déclare qu'ils brûlèrent au moins vingt villages et croit qu'ils mangèrent ensuite les gens qu'ils 
avaient tués. Néanmoins, il ne s'agit guère que d'une supposition, et l'auteur ajoute qu'il n'essaya pas de se renseigner. Le récit de cet incident par Marno (p. 134) ne nous éclaire pas davantage, à mon avis. Bien sûr, il parle des «menchenfressenden Niamniam » : "Als die Mohamedaner ins Land kamen, soll auch bei den Makraka Antropophagie allgemein gewesen, jetzt dürfte dieselbe in dem Seriben-Gebiete (centres administratifs et commerciaux) wohl ziemlich erloschen sein und nur gelegentlich ausgeübt werden. » Il pensait que lorsqu'un peuple était cannibale, il mangeait aussi les chiens, ce que faisaient les Azande. De même Schweinfurth (1873, 11, pp. 10-11) considérait que manger les chiens était le premier pas vers le cannibalisme. Geyer (p. 305) reprenait peutêtre son idée lorsqu'il suggérait une connexion possible entre le cannibalisme et le fait de manger les chiens. On ne s'étonne pas que Potagos ne nous parle guère de cannibalisme, car il reste laconique dans l'ensemble. Ce médecin grec qui visita les Azande de l'Ouest en 1876 et 1877 note qu'ils sont tous cannibales (p. 283), mais n'en donne aucune preuve. Le témoignage de l'officier et cartographe italien Gaetano Casati nous est d'une tout autre utilité, car il séjourna en pays zande ou dans les alentours, d'avril 1881 à août 1884. Il remarque (1891, 1, p. 207) que la chair des morts et des victimes de guerre constitue un plat apprécié, mais ses preuves ne dépassent guère la conjecture et le ouï-dire. Un prince lui aurait avoué $(1, p .177)$ que le goût de la viande de singe ressemblait à celui de la chair humaine. Il est amusant de noter que le roi Wando, qui nous est présenté avec admiration par Schweinfurth et Emin Pasha comme un opposant farouche du cannibalisme, soit dénoncé ici comme un cannibale si avide que lorsqu'il rendit visite à Ringio, chef d'une section des Azande Adio, il lui demanda de la chair humaine, alors que son hôte lui offrait une grande variété de chairs animales : «Oh, mon cher ami, dit-il, après une si longue abstinence, je meurs d'envie de manger de la chair humaine » (I, p. 253). (On peut se demander en quelle langue se fit une aussi remarquable et incroyable déclaration.) Casati ne nous cite qu'un seul exemple précis de cannibalisme (I, p. 297): Durant le siège d'Amadi par les Derviches, soixante-dix Arabes suspectés de sympathie avec l'ennemi furent livrés par le haut commandement aux Azande Bombe (Adio) qui soutenaient le gouvernement, et «après qu'ils eurent égorgé les victimes comme des bêtes, les anthropophages affamés les mangèrent ». Mais Casati ne se trouvait ni à Amadi, ni aux environs à cette époque. D'autre part, on sait que tous ses carnets de notes furent détruits lors de sa capture par le roi Kabarega du Bunyoro, si bien qu'il dût rédiger de mémoire, et on sait aussi qu'il avait tendance à épicer ses récits de sensationnel. Ce que je veux mettre en évidence, c'est que Casati, qui passa quelque trois ans parmi les Azande, dans un pays où le cannibalisme était considéré comme une pratique générale, ne put nous citer un seul cas dont il ait été témoin. Lui non plus n'évoque ni crânes, ni mains, ni pieds, etc.

C'est un autre Italien, Romolo Gessi, gouverneur de la province de Bahr-el-Ghazal au Soudan égyptien, qui ajoute un détail horrible au récit macabre de Schweinfurth. Entre 1878 et 1880, il fut chargé d'anéantir la révolte du leader des esclaves de cette région, Suliman Ziber Bey. Un bon nombre d'Azande ou de gens qu'il prenait pour des Azande, des indigènes amis, venus de l'ouest, étaient rattachés à son armée. Il écrit (1892, p. 255) : «De toutes nos troupes, seuls les Sandeh et les Makrakas restèrent en bonne santé en mangeant de la chair humaine. Aussitôt la bataille, ils coupaient les pieds des morts comme morceaux de choix et ouvraient les crânes dont ils conservaient les cervelles dans des marmites. »Cependant, il ne prétend pas avoir observé lui-même ces actes révoltants. Jamais, il ne s'était rendu au pays des Azande, et sa connaissance de leurs habitudes et de leurs coutumes semble minime. En 1879, peu de temps après la fin de la campagne contre Suliman, deux missionnaires anglicans, le Rév. C. T. Wilson et le Dr R. W. Felkin, rendirent visite à Gessi et ils notent, 
dans leur ouvrage (Wilson et Felkin, 1882, 11, p. 168), que lorsque Gessi fut assiégé à Dem Idris et que ses troupes mouraient de faim, la plupart de ses soldats Niam-Niam mangeaient les morts. Comme ni l'un ni l'autre n'assistèrent à ce siège, ils reçurent cette information de Gessi lui-même. On ne peut donc la considérer comme une confirmation de son propre récit. Aucun de ces deux missionnaires n'était jamais allé en pays zande et la déclaration suivante de Felkin (1882, Il, pp. 187-188) doit être traitée avec une semblable réserve : «Il ne faut pas conclure du fait que les soldats Niam-Niam mangeaient les victimes de guerre, qu'ils n'étaient cannibales qu'en cas de famine ou de guerre. C'était pour eux une habitude normale de manger les morts, et on laissait parfois mourir les enfants afin que parents et amis puissent satisfaire leur horrible penchant. » Comment Felkin avait-il pu recueillir une telle information? Le gouverneur de la province équatoriale fut, à partir de 1878, Emin Pasha (Eduard Schnitzer). Il devint célèbre aussi bien par ses études rigoureuses et détaillées d'histoire naturelle que par sa résistance aux Derviches et sa soi-disant délivrance par Stanley. En 1883, il écrit (Emin Pasha, 1888, p. 206) que les Pygmées Akka mangent de la chair humaine, qu'il en a des preuves, sans dire en quoi elles consistent, que chez les Mangbetu le cannibalisme est si répandu que pratiquement on n'enterre jamais personne et que l'on échange ouvertement les cadavres. Il ajoute que les Mangbetu qui ne mangent pas de chair humaine sont des exceptions « tout comme les Azande qui s'en abstiennent, les chefs Wando et Yangara doivent à ce fait une part de leur réputation ». Il n'apporte aucune preuve confirmant que les Azande sont cannibales et il ne pouvait guère en avoir en dehors de rumeurs, car les seuls Azande avec qui il eut des contacts, superficiels et de courte durée, étaient les Adio, à l'extrémité est du pays zande. Son allusion au roi Wando laisse supposer qu'il basait son affirmation sur l'ouvrage de Schweinfurth plutôt que sur son expérience personnelle.

A cette époque, le mythe était si bien établi que tous les voyageurs qui décrivaient leur passage au pays des Azande se croyaient obligés de parler de leur cannibalisme, bien qu'ils n'en aient personnellement rencontré aucun indice. En réalité, la réputation de mangeurs d'hommes des Azande conférait au voyageur une certaine distinction. Les auteurs, les uns après les autres, répètent cette accusation sans apporter d'autres preuves, quand ils en donnent, que des ouï-dire.

L'officier belge, Florimund Delanghe, aidé d'un grand nombre d'Azande, sujets de Ukwe, le fils de Wando, attaqua en avril 1894 le camp de Renzi, frère d'Ukwe, qui s'enfuit en laissant derrière lui un important butin et une centaine de morts. Laconiquement, Delanghe remarque, sans préciser davantage (Lotar, 1946, p. 192): «Les ennemis tués furent mangés. » Vient ensuite un passage remarquable (Lotar, p. 193). Ukwe lui dit : "Wando est venu me trouver pendant la nuit pour me dire que mes gens devaient absolument couper la tête de Renzi, s'ils le prenaient, pour le punir d'avoir si mal agi envers nous, les Européens, qui avons fait des fils de Wando des gens puissants et heureux. Wando ne veut pas qu'on mange encore les cadavres; il veut qu'on rapporte les têtes au camp comme pièces à conviction. »

Ainsi le grand Wando, mort en 1892 ou 1893, cité comme adversaire du cannibalisme par Schweinfurth et Emin Pasha, comme un de ses adeptes par Casati, fait-il une dernière apparition fantomatique pour s'y opposer. Le capitaine Guy Burrows, officier anglais au service du gouvernement du Congo libre de 1894 à 1897, note qu'il est «confirmé que toute la race est cannibale »(Burrows, 1898, pp. 60-61), que les Azande habitant au sud de la 
rivière Welle laissent leurs femmes et leurs enfants manger de la chair humaine, tandis que les hommes se bornent à manger leurs victimes de guerre. Jusqu'ici les Azande n'étaient que cannibales ; ils ne chassaient pas pour se procurer de la chair humaine (Burrows, 1899, p. 44). Harold Reynolds (un prospecteur ?) mentionne (1904, p. 244) qu'il a entendu de vieux Azande avouer qu'ils avaient mangé de la chair humaine, " mais maintenant cette habitude a disparu ». Enrico Craffen et Edoardo Colombo, deux Italiens dont je n'ai pu retracer la carrière ${ }^{1}$, rédigèrent un compte rendu sur les Azande. Ils y déclarent: les Azande sont cannibales (1906, pp. 769 et 792); d'après eux la chair du chimpanzé est presque aussi savoureuse que la chair humaine (pp. 773, 799), ils traitent bien les prisonniers de guerre dont ils font leurs esclaves, ils ne les tuent que rarement pour les manger, sauf en cas d'incapacité physique pour le travail manuel (pp. 785-786); en temps de paix il est très difficile de les surprendre en train de manger de la chair humaine car ils vont alors au fond de la forêt. « La chair humaine n'aime pas tout le monde, disent les Azande, et cela signifie que certains d'entre eux éprouvent du dégoût à la manger » (p. 792).

Dès le début de ce siècle, des missionnaires catholiques commencèrent à pénétrer à l'intérieur du pays zande. Parmi ceux-ci, celui qui se révèle pour nous le plus important est le vicaire apostolique de l'Afrique centrale, Franz Xaver Geyer, qui, en 1906, partit en reconnaissance missionnaire vers le sud depuis Wau jusqu'au royaume de Tembura, autrefois celui de Ndoruma, fils d'Ezo. Dans son ouvrage, dont il semble avoir emprunté bien des données à Schweinfurth, il écrit (1914, p. 305) : "Dass der Ruf der Njam Njam als Menschenfresser begründet ist, steht ausser Zweifel. Den gleichen Ruf haben die Pambia und Abarambo. Aber vorzugsweise gelten die Njam Njam als das Volk der Menschenfresser mit Auszeichnung. Mehrere haben mir frei erklärt, das sie Menschenfleisch gegessen, das sie es aber seit Ankunft der Engländer nicht mehr getan haben. Andere bestätigten, das dieses Fleisch schmackhaft sei und besonders das vom Schenkel und Oberarm. Einer bekannte, dass bei ihm zu Hause erschlagene Feinde ganz gebraten und die Brusthöhle mit Bananen ausgefüllt worden seien. Die meisten jedoch antworteten ausweichend oder leugneten es offen, da sie merkten, dass wir es für eine verabscheuungswürdige Sitte halten, oder weil sie gar fürchteten dass sie nachträglich dafür zur Rechenschaft gezogen würden. Fest steht, dass früher die Menschenfresserei ihnen keinen Abscheu eingeflösst liait, und dass jetz öffentlich niemand mehr es wagt. » Geyer ne s'aventure pas à déterminer les motifs du cannibalisme : besoin de viande ou rite religieux ? Mais il fut frappe que ses informateurs insistassent sur le fait que l'on mangeait les ennemis tués ou capturés à la guerre, et qu'en conséquence il pouvait s'agir d'un acte de vengeance; peut-être encore d'une espèce de sacrifice où surnaturel et naturel se mêlaient.

Carmignani cite (1954, pp. 41-43) des missionnaires catholiques qui parlent des Azande dans les premiers numéros de La Nigrizia que je n'ai pu consulter. L'un de ces missionnaires est le même P. Geyer qui semble alors (1906) beaucoup moins convaincu du cannibalisme zande (peut-être était-ce avant son voyage au pays zande). Voici ce qu'il écrit : "Sulla verità o no di questo fatto cercai spesso d'informarmi; ma è difficile sapere qualche cosa di positivo. Ormai conoscendo essi che questo è tenuto per un costume barbaro, non Io confessano più, quand'anche Io avessero avuto. Solo dalle gente estranea ai Niam-Niam, ai Bambia ed agli Abarambo, giacchè tutti e tre questi popoli godono fama d'esser cannibali, si

M. A. Lecointre a découvert depuis que E. Craffen était officier de la Force publique. Un certain O. G. Colombo (est-ce le même ?) était sous-officier dans la même Force publique. 
pub saperne qualche cosa. Mi lu detto che i detti popoli avevano ed hanno ancora il costume di mangiare i nemici uccisi, se Io possono fare di nascosto. Credo però che oggidì sotto il governo nessuno osi farlo. » Un autre père de Vérone, P. Moroni, dit que leurs missionnaires peuvent affirmer que : "è difficile trovare un Niam-Niam di una certa età, che non abbia almeno assaggiato un qualche pezzo di coscia e spolpato una costola umana. Naturalmente il Governo anglo-egiziano ha fatto ogni sforzo, per far scomparire questa vergogna ; per cui il cannibalismo va facendosi sempre più raro; anzi credo possa dirsi senz'altro scomparso ». Le P. G. Bertola (1912, p. 102) qui connaissait bien les Azande les appelle, de même que le P. F. Zattoni (1913, pp. 43-47, 58-62, 72-76), « cari cannibali ».

Quant à la valeur à accorder à ces sortes d'indices, il n'est qu'à consulter le récit de Mgr Geyer sur la manière dont il jugea bon d'aborder le problème du cannibalisme chez les Azande. Un jour qu'il entrait avec sa suite dans une colonie zande au nordouest de Wau (1914, p. 109), « fragten wir diese ersten Njam Njam, die uns gegenübertraten, scherzend, ob sic Menschenfleisch essen Zähnefletschend und faut lachend versicherten sic in gebrochenem Arabisch «Essen Menschen keine » ». Il n'est pas étonnant qu'ils rirent aux éclats en entendant cette curieuse salutation, d'autant qu'il s'agissait d'Azande expatriés qui se considéraient certainement comme évolués. Plus remarquable encore (1914, p. 194) fut son entrevue avec Bujo, un Zande qui vivait à l'époque dans une communauté villageoise zande, près de Wau. C'était un garçon d'une quinzaine d'années, au teint cuivré, aux membres déliés et au visage agréable. "Auf die Frage, ob er Menschenfleisch geniesse, entgegnete er entrüstet : "Wir gehorchen der Regierung und tun es nicht, nur die anderen « Njam Njam tun es. » » Ce charmant et svelte garçon, non seulement se considérait comme évolué - il avait pris un nom arabe, Hasan - mais était aussi un aristocrate parmi les aristocrates. C'était le fils de Mvuto, lui-même fils aîné de Ndoruma, le plus célèbre des fils du roi Ezo, lui-même fils du roi Bazingbi. On ne s'étonne pas qu'il ait répondu avec indignation à ce qu'il estimait être une question insultante.

Le capitaine R. G. Anderson du Corps médical de la Royal Army rédigea un compte rendu sur les Azande de la région Meridi du Soudan anglo-égyptien, qui ne constituent qu'une minorité au milieu d'un grand nombre de peuples étrangers auxquels ils sont mêlés (Baka, Mundu, Avokaya, «Jur », etc.). Le travail du capitaine Anderson (lequel est devenu par la suite un de mes bons amis) reste vague. Son auteur mal informé, en dehors de ses observations purement médicales, se montre dépourvu de sens critique en matière ethnographique. Il note (Anderson, 1911, p. 240) que les Azande ont des habitudes cannibales auxquelles s'adonnent les habitants du Congo belge et il mentionne (p. 274) la mort du capitaine Roenning, alors commandant de Kiro et de Lado (administrés parles Belges à cette époque) qui, en se rendant à Boma en 1908, fut capturé, « dépecé et mangé cru » (il cite un «rapport officiel » sans donner la référence; il ne dit pas non plus où, comment, pourquoi et par qui, fut dévoré le malheureux capitaine). En dehors de ce cas incertain, il n'apporte aucune preuve justifiant que les Azande étaient alors ou furent jamais cannibales, et il admet la difficulté d'obtenir une information exacte à ce sujet. Il dit vaguement que les individus tués, blessés ou capturés lors des raids, ainsi que les personnes de tribu zande sur le point de mourir, étaient mangées. Il pense que, selon les croyances indigènes, consommer la chair de ses ennemis rendait celui qui la mangeait féroce, belliqueux et sage, mais ne fournit pas la raison qui l'a conduit à cette supposition. Il suggère une relation possible entre cannibalisme et homosexualité. L'histoire du capitaine Roenning fut reprise par un fonctionnaire britannique, Basil Spence, qui prétend aussi (1920, p. 300) 
que les Belges et les Français relatèrent un nombre incalculable de cas de cannibalisme et que l'habitude de manger de la chair humaine avait été donnée par les Ambomu aux peuples qu'ils avaient conquis, et non l'inverse. Mais nous ne devons pas prendre trop au sérieux le témoignage d'un fonctionnaire qui écrit (Spence, 1920, p. 301) : «Les Azande de tous rangs admettent invariablement, parfois volontiers, parfois après de nombreuses questions, qu'autrefois leur tribu pratiquait le cannibalisme. Mieux, des centaines d'entre eux admettent effectivement avoir mangé de la chair humaine avant les deux dernières décennies. » Certains voyageurs se contentent de remarquer, comme le capitaine von Wiese (Mecklenburg, 1913, p. 210), que les Azande sont « les plus dangereux cannibales d'Afrique centrale », ou comme sir C. N. E. Eliot (1902, p. 618) que les Azande Makaraka « ont la réputation d'être cannibales, mais qu'ils sont braves et utiles en tant que soldats ».

J'ai maintenant cité tous les voyageurs et premiers administrateurs les plus importants. Avant de tenter de tirer quelques conclusions de ces récits, nous examinerons les témoignages de ceux qui vinrent plus tard et qui, dans leurs écrits, parlent surtout des Azande : les administrateurs, missionnaires, ethnologues et anthropologues. Puisqu'il est reconnu que les pratiques cannibales cessèrent, sauf peut-être secrètement, après l'établissement de l'administration européenne, ces observateurs n'étaient donc plus à même d'observer des preuves tangibles de leur existence comme l'avaient pu les premiers voyageurs, qu'ils en aient découvert ou non. Mais d'un autre côté, en ce qui concerne les preuves orales, les derniers venus eurent l'avantage, ou bien de séjourner longtemps parmi les Azande, ou bien de parler leur langue avec une certaine facilité, ou bien de pouvoir poursuivre leurs recherches à la lumière d'une meilleure connaissance ethnologique et anthropologique, ou bien de réunir plusieurs de ces possibilités à la fois.

L'ethnographe polonais, Jan Czekanowski, qui explora cette région d'Afrique en 1907 et 1908, écrit (1924, p. 30) : " Autrefois les aristocrates considéraient la chair humaine comme une part importante de leur menu. Même aujourd'hui, le cannibalisme n'a pas complètement disparu chez les Azande quoique les Européens fassent de leur mieux pour le supprimer. » Il ajoute qu'ils ne mangeaient pas les membres de leur propre tribu, mais seulement les prisonniers de guerre et les esclaves, bien qu'on lui ait rapporté que les Angbai, et eux seulement, étaient supposés manger leurs propres morts. Il ajoute encore que « les Azande du Nord, ceux qui vivaient le long du Bomu (Mbomu), avaient cessé leurs pratiques cannibales, disait-on, avant l'arrivée des Européens ». On peut se demander pourquoi ? (Nilis et de la Kéthulle confirment son observation : ils notent que les Banjias - la branche Abandiya des Azande - n'étaient pas cannibales (Anon., p. 428), mais il semble que Potagos la contredise (1885, p. 295), bien qu'on ne sache pas très clairement de qui il parle.) Dans un autre passage (p. 52), Czekanowski note que, outre les esclaves et les prisonniers, on mangeait peut-être les gens isolés et sans défense. Il cite le cas de deux princes, Bokoyo et Zunet, que l'on tenait pour particulièrement coupables de cannibalisme. Il mentionne encore (p. 112) que les femmes n'auraient pas été admises à manger de la chair humaine. Administrateur au Congo belge, de Calonne-Beaufaict, qui mena avec tant de vitalité et d'enthousiasme ses recherches ethnographiques, n'aborde pas le sujet. Un autre Belge, soldat de carrière, Armand Hutereau, qui servit l'Etat libre du Congo de 1896 à 1909 et de 1911 à 1912, conduisit une mission ethnologique. Avant de le citer, je voudrais attirer l'attention sur les déficiences de sa description des modes de vie zande : il était meilleur ethnologue qu'ethnographe. Cependant, il ajoute, sur le cannibalisme, quelques détails curieux que les autres ne mentionnent pas ; il serait intéressant de savoir d'où il les tient. Il écrit (Hutereau, 1909, p. 37) que les Azande 
avaient l'habitude de mutiler les cadavres de leurs victimes ennemies, de couper la main droite et les parties sexuelles des hommes, et de les emporter avec eux. Les parties génitales servaient dans la fabrication d'une pommade à laquelle on attribuait de grandes vertus. Il continue : "Les Azande sont cannibales. Quand ils ont pu se procurer un fusil, ils s'abstiennent de chair humaine, persuadés qu'en en mangeant ils détruiraient toutes les vertus et toutes les qualités de leur arme. La chair des ennemis tués à la guerre est rapportée au village pour faire ripaille en compagnie des indigènes de tribus amies, alliées ou soumises, dont les bandes azande sont presque toujours accompagnées. » Il nous signale que les Azande Adio mangeaient les prisonniers exécutés, mutilaient les corps des ennemis tués à la guerre et qu'au moment où il écrivait, on pratiquait encore secrètement le cannibalisme (p. 58). Tout ceci n'est que ouï-dire.

Parmi les descriptions des Azande, l'une, sans conteste, des plus dignes de foi est celle de mon ami Mgr Lagae, dominicain qui passa bien des années chez les Azande, et le premier de nos témoins à pouvoir facilement converser avec eux dans leur propre langue. Dans un passage sur le sort des esclaves, il parle de cannibalisme (1926, pp. 49-50). Après avoir cité les propos de von Wiese, Anderson, Milz, sur le cannibalisme des Azande, il donne son point de vue : «Les Azande ont certes dans leur langue un terme qui désigne la chair humaine : kawa, et les mangeurs de chair humaine : alikawa. Il y aurait toutefois une forte exagération à dire que tous les Azande sont ou furent anthropophages. Les Avongara ne le furent certainement pas. On ne peut perdre de vue que ceux que nous appelons les Azande sont en somme un agglomérat de peuplades assez diverses. Les Avongara sont d'accord pour dire que le groupe primitif zande a toujours détesté l'anthropophagie. Parmi les peuplades acculturées, d'origine étrangère, quelques-unes n'ont pas renoncé à leur prédilection pour la chair humaine, qu'ils avaient jadis. Nous pouvons citer les Azande Bamboy du Nord-Est au Soudan, les clans étrangers (auro) des Abunge et des Asibale, les Basiri et les Apambiya, ainsi que quelques clans parmi les Abandiya, sans oublier les clans d'origine Madi et Barambo. Le groupe primitif zande avait l'anthropophagie en horreur. » Pour la première fois, le cannibalisme des Azande est considéré par rapport à l'histoire et par rapport aux différentes ethnies qui composent l'amalgame zande. Mon vieil ami, le major P. M. Larken, qui vécut de nombreuses années parmi les Azande et qui parlait couramment leur langue, écrit (1927, p. 115), dans le même sens que Mgr Lagae : «Les purs Azande dénient que leurs ancêtres aient été cannibales et disent que seules l'étaient les tribus qu'ils avaient conquises. La grande tribu des Abarambo mangeait certainement de la chair humaine autrefois ; ses membres l'admettent d'ailleurs, mais ils ajoutent n'avoir jamais mangé quelqu'un de leur propre famille. Ils accusent les Abangminda d'être également cannibales en précisant que ces derniers creusaient, dans leur maison, un trou qu'ils recouvraient de feuilles, puis ils invitaient un passant à s'asseoir à cet endroit ; lorsque celui-ci était tombé dans le trou, ils versaient de l'eau bouillante pour le faire mourir. Mais ce me semble une manière si compliquée de se procurer une victime que l'histoire est à peine vraisemblable. » Dans ce passage, le major Larken répète ce qu'il avait écrit quelques années auparavant (1920, p. 78). Craffen et Colombo, dont j'ai déjà cité le témoignage, vont dans le même sens que Lagae et Larken quand ils écrivent (1906, p. 792) que les Azande refusent catégoriquement qu'on les appelle Niam-Niam ou, ce qui est la même chose, auro, étrangers ; et quiconque les appelait ainsi « courait le risque de se voir assailli ou tout au moins de s'entendre répliquer, « où m'astu vu manger de la chair humaine »?» 
C'est entre les années 1927 et 1930, pendant quelque vingt mois, que j'effectuai mes propres recherches parmi les Azande, presque entièrement dans cette partie du vieux royaume de Gbudwe qui devint la région administrative de Yambio. Je n'ai publié que deux passages relatifs au cannibalisme. Le premier (1937, p. 292) est un texte qui raconte comment, à la mort de Gbudwe, son fils Gangura exécuta l'oracle de son père, Mbikogbudwe, de peur qu'il ne livre les secrets de son défunt maître. Lorsqu'il fut tué, deux hommes, Bangozanga du clan Abamburo et Ngbani du clan Agiti, demandèrent le cadavre à Gangura qui le leur accorda. Ils le coupèrent en deux, puis en prirent et en mangèrent chacun la moitié. "Ainsi Bangozanga mangea l'oracle de Gbudwe, un très beau Baka à la peau noire et aux belles dents blanches. » L'autre allusion au cannibalisme est un texte $(1956$, p. 73$)$ dicté par le même informateur que celui qui me renseigna sur le jeune et beau Baka, Kuagbiaru, témoin digne de foi, très au courant des affaires publiques avant l'établissement de l'administration européenne en 1905. Le texte rapporte qu'autrefois les Azande étaient comme des bêtes de proie, comme des lions, des léopards et des chiens sauvages qui mangent la chair humaine. On y décrit comment ils la préparaient et la mangeaient, réservant à cet usage une marmite spéciale. Les gens qui mangeaient de la chair humaine la mangeaient entre eux. On mangeait un homme, tout simplement parce que « c'était de la bonne viande ». Kuagbiaru avait observé des pratiques cannibales dans le clan des Akpura, dans le clan des Agiti, dans le clan des Abamburo et dans beaucoup d'autres, "car, autrefois, presque tous les Azande mangeaient des hommes ». Kuagbiaru était précis et catégorique dans son affirmation. Cependant certains trouvaient cette coutume répugnante et horrible et se moquaient des mangeurs d'hommes. C'était une affaire d'habitude : ceux dont les ancêtres étaient cannibales l'étaient aussi. Je citerai encore un texte noté sous la dictée d'un témoin oculaire : «Durant la guerre contre les Derviches, les Azande en mangèrent beaucoup. Ils installaient des sortes de plates-formes pour mettre la chair à sécher. Le camp empestait la chair humaine. Ils mangeaient les Derviches parce qu'ils leur faisaient la guerre. Comme les soldats en consommaient une grande quantité, on trouvait de la chair humaine tout autour du camp des princes. Seuls les nobles s'en abstenaient. »

J'ajouterai que le travail de mes professeurs, C. G. et Brenda Z. Seligman, était presque entièrement basé sur mes notes personnelles, bien qu'ils le rédigèrent dans leur propre style. Ils n'avaient jamais visité le pays zande. Ils notent (1932, p. 497) que les Avongara, l'aristocratie régnante, ne pratiquèrent jamais le cannibalisme, que dans le peuple c'était une affaire de goût personnel, que l'approvisionnement en chair humaine dépendait de la guerre, que les Ambomu (les Azande véritables, distincts des éléments étrangers assimilés) avaient certainement été cannibales dans le passé - soixante ou soixante-dix ans avant que M. et Mme Seligman écrivent - et qu'Ongosi, un de mes amis avait vu, dans sa jeunesse, des paniers de bras et de jambes humains préparés pour être mangés.

Voici ce que l'on sait, pour le moment, du cannibalisme zande. Citer d'autres auteurs ne servirait qu'à augmenter le nombre des déclarations sans renforcer les preuves. Quelques-uns d'entre eux sont nommés dans un petit ouvrage que Renzo Carmignani a consacré au cannibalisme zande: Il Cannibalismo degli Asandè (1954), avec pour sous-titre: Un problema controverso dell'Etnografia africana, ainsi que dans un article intitulé « Gli Asandè o Niam-Niam e il problema del loro cannibalismo » (1955), qui recoupe en grande partie l'ouvrage ci-dessus. Dans cet article, Carmignani fut le premier à mettre l'accent, comme nous le verrons plus loin, sur les aspects juridiques et ce qu'il croyait être les aspects magiques du cannibalisme zande. 
Personne ne niera, je pense, qu'examinées séparément, les preuves fournies par les voyageurs sont, ou bien douteuses, ou bien sans valeur ; mais il n'y a pas de fumée sans feu, et si nous les considérons dans leur ensemble, nous pouvons conclure qu'il est très probable que le cannibalisme fut pratiqué au moins par certains Azande. En outre, j'estime assez concluants les témoignages d'hommes tels que Lagae et Larken, bien que fondés non pas sur des observations personnelles, mais sur des faits rapportés. J'ajouterai mes propres informations inédites. Ce que je considère comme décisif, c'est qu'aucun ancien Zande de ma connaissance, j'entends par là un homme bien informé, une personne digne de foi, n'a jamais nié, sauf en ce qui le concerne, en dépit de l'embarras parfois ressenti, que des hommes aient été mangés autrefois. Tous cependant s'accordent à dire qu'on ne mangeait que les victimes de guerre et les criminels; mais j'ai aussi entendu dire qu'en temps de famine, certains hommes sont supposés avoir échangé leurs filles pour qu'elles soient mangées (il s'agit là, bien entendu, d'une tradition historique et personne ne prétend avoir été témoin d'un tel échange); on m'a aussi raconté que parfois on tuait et mangeait un gros Buguru, plus ou moins hors la loi, membre d'une tribu étrangère assujettie. Si nous poussons plus avant, et si nous nous interrogeons sur le nombre de gens qui furent mangés, le nombre de ceux qui mangeaient de la chair humaine, la fréquence de ces festins, l'identité des victimes et celle des cannibales, les questions restent sans réponse précise. Les textes cités conduiraient à croire à la pratique générale, mais, quiconque a opéré des recherches sur le terrain n'ignore pas le risque de généraliser à partir de quelques incidents. Aussi, quand on m'eut dit que les gens avaient l'habitude de manger de la chair humaine, je cherchai à découvrir, à travers l'expérience personnelle de mes informateurs, qui, en fait, en avait mangé. Au cours de discussions sur ce sujet, je n'ai réuni, dans le royaume de Gbudwe, qu'une quinzaine de cas où l'on avait réellement vu couper des cadavres destinés à être mangés. Il y eut les deux sujets de Kipa, petit-fils de Gbudwe, qui mangèrent le jeune et beau Baka, puis Zangwa de la tribu des Abangbinda, qui mangea de la chair humaine lors de la guerre de Gbudwe contre le roi Ngangi, Maikondo (du clan akowe) qui mangea les cadavres des victimes lors de la guerre contre les Derviches. Maikondo était chef de l'une des compagnies militaires royales, et Gbudwe lui avait donné un couteau de cérémonie qui l'autorisait à prendre ses repas dans la cour intérieure, mais il en fut exclu par ses camarades, car ses habitudes cannibales « gâtaient les marmites de Gbudwe ». On avait vu deux hommes, Iyanda (du clan akowe) et Nzagu (du clan agiti), manger des morts lors de la guerre de Gbudwe contre son neveu Renzi à Birisi. Il y en avait d'autres: Ginegate (du clan abanzuka), Naparakondo (du clan agbutu), Buliya (du clan akpura), qui tous mangèrent des Derviches tués à la guerre, de même que Basuru (du clan amuzungu) qui portait, dit-on, un collier de dents humaines (kpakasa meme linde aboro), et quelques autres. Je n'ai pas procédé à des recherches approfondies sur le cannibalisme. Les cas notés étaient ceux dont avaient été témoins quelques individus, les plus âgés parmi mes informateurs les plus intimes, dans une partie seulement du pays zande. Si j'avais poussé mes recherches, si j'avais interrogé un plus grand nombre d'informateurs, sur une plus vaste étendue, il est probable que d'autres noms auraient pu s'ajouter à la liste. Même alors, je ne crois pas qu'on aurait dépassé une fraction minime de la population. Si le cannibalisme avait été une habitude régulière et courante, mes informateurs n'auraient pas cité ces exemples isolés comme des épisodes horribles du passé. Mais il est vraisemblable aussi qu'un individu qui a mangé des morts une fois a pu récidiver. Si tout le monde, ou si la plupart des gens, ou même si un bon nombre d'entre eux mangeaient les morts lors des campagnes, pourquoi ne mentionner qu'un nom ou deux ? Ceci nous laisse supposer qu'on ne mangeait les morts qu'exceptionnellement. D'autre part, si, comme on le prétend et comme il 
est vraisemblable, on ne mangeait que les victimes de guerre et les criminels, le cannibalisme ne pouvait être une pratique régulière et universelle. De surcroît, si les habitudes cannibales avaient été universelles, les voyageurs comme Junker et Casati, qui séjournèrent le plus longtemps au pays zande, en auraient certainement remarqué des indices précis. Enfin, si le cannibalisme avait été habituel et largement répandu, on ne voit pas pourquoi il aurait cessé dès le début de l'occupation européenne, tout au moins tant que la répugnance des Européens ne se fût manifestée par des sanctions. Pourtant, je n'ai vu aucun rapport attestant qu'on ait condamné un individu pour cannibalisme. Je n'ai trouvé aucune déclaration d'officiers belges, à part celle de Delanghe déjà citée, mentionnant que quelqu'un ait été mangé au cours des sanglantes campagnes menées au Congo belge contre les Azande ou avec eux. Ces campagnes devaient pourtant offrir d'excellentes occasions de festin pour les cannibales.

Même en admettant l'existence de pratiques cannibales, au moins en certains cas, il est difficile de déterminer l'origine ethnique des cannibales, étant donné la complexité de l'amalgame social et politique zande, comme l'a si justement noté Mgr Lagae. Celui-ci et Larken, ne l'oublions pas, affirment que l'aristocratie vongara n'était certainement pas cannibale (en dépit des dires de Piaggia, Casati et Czekanowski), que les Avongara et les «purs » Azande prétendent que les premiers Azande (les Ambomu, par opposition aux éléments étrangers qui s'y sont mêlés) n'étaient pas cannibales, à l'inverse des étrangers et de certains peuples voisins. Selon nos sources, auraient été cannibales: les Mangbetu, les Ababua, les Akare, les Bamboy, les Abunge, les Asibali, les Abaka, les Basiri, les Apambia, les Nzakara, certains clans des Abandiya, les Amadi, les Abarambo, les Babukur (Abuguru), les Abangbinda, les Huma, les Mabode, les Abangba, probablement les Mundu, les Pygmées et les Mobanghi. Aujourd'hui, le témoignage des Azande confirme, en bien des cas, celui des Européens. En d'autres termes, de nombreux peuples, qui contribuèrent à former la nation zande d'aujourd'hui, ont été accusés de cannibalisme. Nous nous trouvons en face des mêmes difficultés que celles auxquelles nous nous sommes confrontés lorsque nous avons tenté de peser les accusations contre les Azande en général. Ici encore, elles se fondent sur de simples ouï-dire et sur de simples affirmations. Malgré tout, le témoignage des Azande confirme si bien les dires de Lagae et de Larken, que je serais prêt à accepter, comme très probable, que certains de ces peuples étaient cannibales, qu'ils continuèrent leurs pratiques, lorsqu'ils en eurent l'occasion, au cours du processus d'assimilation sous l'autorité vongara, que le groupe des Avongara-Ambomu n'était pas cannibale bien que quelques-uns de ses membres aient adopté la coutume étrangère. Ainsi se trouvent conciliés les dires des voyageurs en général, ceux d'écrivains plus récents et ceux des Azande eux-mêmes. Si l'on attribue au terme Azande son sens restreint d'Azande d'origine, c'est-à-dire d'Avongara-Ambomu, les Azande n'étaient pas traditionnellement cannibales, mais si l'on entend par Azande tous les peuples maintenant assimilés et classés sous ce nom, alors on peut affirmer que les Azande étaient cannibales dans une certaine mesure. Les faits que nous connaissons nous conduisent eux aussi à cette conclusion, car il n'est pas possible d'expliquer, dans une société, l'existence de deux sentiments si différents vis-à-vis du cannibalisme, sans tenir compte des divergences d'ethnies et de classes. Nous saisissons maintenant le pourquoi de certaines divergences d'opinion entre nos auteurs ainsi que de certaines contradictions apparentes dans les récits eux-mêmes. Par exemple, Piaggia parle de cannibalisme à la Cour et dans le royaume du roi Tombo, mais n'y fait pas allusion dans ses autres voyages au pays zande. A cette époque, les sujets du roi Tombo devaient être surtout des étrangers, principalement des Babukur. Ce peuple, en partie désagrégé par l'incorporation dans son armée d'éléments amadi et abarambo, n'est pas, aujourd'hui encore, complètement assimilé. Quant à Schweinfurth, la 
question est de savoir s'il entra en contact avec beaucoup de purs Azande. Il en vit certainement quelques-uns, mais il traversa une région qui, maintenant encore, est en majorité peuplée d'éléments d'origine étrangère, donc à plus forte raison à cette époque, et, qui plus est, d'étrangers comme les Abarambo, les Amadi, les Abangbinda, etc., qui ne devaient être alors que très partiellement assimilés culturellement et socialement, et qui conservaient maintes habitudes et coutumes d'autrefois. Même si nous ratifions l'exactitude de ses observations, il est évident que Schweinfurth n'avait pas conscience de la grande complexité ethnique du peuple zande de cette région, et qu'en conséquence, il ne pouvait opérer la distinction entre les vrais Azande et les peuples soumis. Ce qu'il dit des habitations nous laisse supposer qu'il s'agissait des cases d'étrangers. En effet, les poteaux (ambama) qui se dressaient devant elles et sur lesquels on pendait des crânes humains et d'autres trophées étaient, en fait, bien qu'il l'ignorât, des monuments érigés aux esprits par les étrangers, auro, mais non par les vrais Azande qui en élevaient de très différents. En outre, les Azande m'ont formellement assuré que c'était l'habitude du peuple cannibale amadi d'accrocher les têtes des victimes tuées à la guerre aux poteaux décrits par Schweinfurth. Ces répartitions ethniques peuvent expliquer de la même façon que Schweinfurth insiste sur les crânes humains, les pieds et les mains pendus aux arbres, alors que Junker, entre autres, n'y fait pas allusion. Junker séjourna au centre et à l'ouest du pays zande. Lorsqu'il traversa la partie est qu'avait visitée Schweinfurth, il avait hâte d'atteindre le Nil ; de graves désordres troublaient alors le pays après une attaque égyptienne contre le royaume de Gbudwe. Il nous faut donc, en examinant les récits des divers auteurs, non seulement nous préoccuper de la valeur de leurs observations, de leurs possibilités d'enquête, mais aussi de leurs itinéraires.

Une telle circonspection parait élémentaire à qui connaît la complexité ethnique des Azande. C'est d'ailleurs un sujet sur lequel le F. Giorgetti, qui vécut plus de trente ans parmi les Azande, critiqua Carmignani, et sur lequel j'ai moi-même insisté (1955, p. 203). Voici ce que dit Giorgetti du principal observateur Schweinfurth. Après avoir signalé (p. 181) que sa description d'une case niam-niam à Diamvonoo, près de laquelle on remarquait des crânes humains suspendus à un arbre mort, présente les caractéristiques de l'habitat madi et mangbetu et non zande, il poursuit : «In tutte le altre illustrazioni nel libro del grande scienzato noto : le lance di guerra proprie degli Amadi, dette « Andigbo » e adottate poi dagli Azande ; gli spilloni per i capelli, di fibule umane, dette Nguma in lingua Madi (termine poi entrato nella lingua Zande e applicato alle fibule di scimmia); le collane di denti di cane e di babbuino, e quelle di avorio lavorato, fatte tutte secondo il costume Barambu-Madi ; le capanne a cono molto alto, in uso ancora ove trovansi degli Abarambu, anche se azandeizzati. Il costume del vestito di pelli, appese alla cintola, che egli descrive, è costume Barambu. Il denti incisivi limati a punta erano costume Madi-Barambu e di altri ex-cannibali Bantu. Gli Azande si distinguono dagli altri popoli per l'usanza che hanno di limarsi, a V rovesciata, solo i due incisivi superiori di mezzo. Le poche parole che Io Schweinfurth riporta corne lingua Niam Niam, sono realmente quasi tutte di lingua Barambu e non Zande. L'Esploratore passò dunque, come egli stesso ammette a oriente del territorio Zande, ove vivevano propio gli ex-Arambu ed ex-Amadi; e questo da sè spiega tutte le scene di cannibalismo di cui fu testimonio. Lo Schweinfurth passé nel vero ex-territorio dei Niam Niam più feroci, i quali, anche se azandeizzati e sotto capi Vungara che cercavano di impedire il cannibalismo abituale, non potevano in pochi anni disabituarsi da vizi secolari. »

Giorgetti note également que la région où Schweinfurth rencontra des signes de cannibalisme n'était pas, à cette époque, ous le contrôle des Avongara, mais sous celui des 
représentants de son ami nubien 'Abd al-Samad. Il conclut que les cannibales dont Schweinfurth et d'autres font mention n'étaient pas des Azande au sens strict du terme, mais des Amadi et des Abarambo soudanais ainsi que des peuplades bantu comme les Bangbinda, les Babukur et les Huma. Lorsque les Ambomu-Azande eurent conquis et absorbé ces peuples qui devinrent alors des Azande désignés sous le sobriquet commun de Niam-Niam, on conféra aussi aux conquérants la réputation de cannibales comme par antonomase. Pour différentes raisons, je ne suis pas totalement l'explication de Giorgetti. Par exemple, il est extrêmement difficile de déterminer avec certitude, dans la complexité culturelle zande, l'apport du peuple zande ou mbomu d'origine et l'apport des races étrangères. La région sur laquelle 'Abd al-Samad exerçait quelque contrôle avait subi, plusieurs années avant la visite de Schweinfurth, la domination des Avongara et le représentant d"Abd al-Samad était un prince vongara. De plus, si incontestablement la population de cette région se constituait en majorité d'éléments étrangers, elle incluait certainement aussi de vrais Azande, et rien ne nous assure que les éventuels actes de cannibalisme soient imputables aux étrangers, aux purs Azande ou bien aux deux à la fois. Néanmoins, l'argumentation de l'auteur paraît valable et va dans le sens de l'opinion zande rapportée par Lagae et Larken. Je suis 'aussi en accord avec ses principales conclusions, à savoir qu'en pays zande le cannibalisme était une habitude rare, qu'il n'était généralement pratiqué qu'en temps de guerre et, probablement, surtout par les étrangers. Comme Carmignani soutient aussi ce point de vue (1957, pp. 397-399), les critiques de Giorgetti n'étaient pas nécessaires.

Si nous pouvons conclure que certains individus, une petite minorité, mangeaient de la chair humaine, mais seulement en certaines occasions rares et précises, il nous faut encore nous poser la question de savoir pourquoi ils en mangeaient. A mon sens, nous devons accepter les déclarations des Azande, transmises par nos meilleurs auteurs et confirmées par mes propres recherches : en temps normal, on ne mangeait que les victimes de guerre et ceux qu'on exécutait, c'est-à-dire des individus sans statut juridique et sans famille pour les défendre. Carmignani (1954, p. 64) dégage cette idée avec pertinence, mais lorsqu'il tente d'expliquer le cannibalisme des Azande par leur mentalité magique et par leur désir de s'approprier les qualités du mort, les preuves lui font défaut. Personne ne fait écho à la déclaration de Hutereau selon laquelle les parties génitales des victimes ennemies étaient utilisées dans la fabrication d'une pommade à laquelle on attribuait de grandes vertus ; son récit même ne suggère pas qu'aucune vertu puisse s'acquérir en mangeant la chair des morts, au contraire, il rapporte que les pratiques cannibales détournaient de son but, disait-on, la balle du tireur. Cependant, aucun autre auteur, sauf Anderson et peut-être Geyer, mais y compris ceux qu'avantageait leur connaissance de la langue zande, ne laisse supposer que le cannibalisme ait eu un caractère rituel magique de quelque espèce. Ces auteurs montrent clairement, qu'à leur avis, la chair humaine était consommée seulement en tant que viande ; certains insistent sur le fait que, dans les campagnes, les guerriers avaient souvent très faim. J'ajouterai que j'ai fréquemment discuté du problème avec des Azande d'un certain âge et que, pour expliquer le cannibalisme, ils ne m'ont jamais donné d'autres raisons que la faim ou, plus souvent, une prédilection pour la chair humaine.

En prenant le terme Niam-Niam comme équivalent de Azande au sens le plus large, nous pouvons maintenant dresser un tableau des informations examinées. J'ai déjà essayé d'en tirer les conclusions et d'en dégager la valeur. Parfois, il faut plus ou moins deviner la pensée d'un auteur. Lorsque aucun motif d'explication du cannibalisme n'est donné, j'ai noté « viande ». 


\section{Qui mangeait?}

M. et Mme

PETHERICKS :

PIAGGIA :

PONCET :

HEUGLIN :

SCHWEINFURTH :

JUNKER:

CHAILLÉ LONG :

MARNO :

POTAGOS:

CASATI:

GESSI :

FELKIN

SCHNITZER (Emin

Pasha) :

BURROWS:

REYNOLDS:

CRAFFEN et

COLOMBO :

ANDERSON :
Tout le monde (y compris les Abarambo).

Les guerriers, le roi Tombo et ses femmes.

Les guerriers (Makaraka)

?

Presque tout le monde.

Habitude courante.

Habitude courante (Makaraka).

Tout le monde (Makaraka).

Tout le monde.

Habitude courante.

Habitude courante.

Habitude courante.

Presque tout le monde.

Tout le monde.

Habitude courante.

Quelques personnes.

Habitude courante.
Les victimes ennemies, les esclaves repris, les vieillards, tout individu sur le point de mourir.

Les victimes de guerre, les criminels.

Les victimes de guerre.

$$
\text { ? }
$$

Les victimes tuées à la guerre,

notamment les vieillards ; les solitaires,

les sans défense.

Les victimes de guerre.

Les victimes de guerre.

? N'importe qui.

Les morts, les victimes de guerre.

Les victimes de guerre.

Les victimes de guerre, tous les morts, les enfants qu'on laissait mourir.

? N'importe qui.

Seulement les victimes de guerre.

Occasionnellement les prisonniers de guerre.

Les victimes et les prisonniers de guerre, les malades.
Viande.

Vengeance, viande, inspirer la crainte.

Viande.

Viande.

Viande.

Viande.

Viande.

Viande (les cannibales mangent aussi les chiens).

Viande.

Viande.

Viande.

Viande.

Viande.

Viande.

Viande.

Viande.

Manger les ennemis rendait féroce, etc. (accompagne l'homosexualité). 


\section{Qui mangeait?}

Qui était mangé ?

Pour quelle raison?

GEYER :

\section{MORONI \\ BERTOLA : \\ ZATTONI:}

CZEKANOWSKI :

HUTEREAU :

LAGAE:

LARKEN:

EVANS-PRITCHARD : Quelques individus, mais non les les Ambomu ; une telle pratique en révoltait

GIORGETTI: Avongara, ni, à quelques exceptions près, certains.

Habitude courante (y compris chez les Pambia et les Abarambo).

Tout le monde.

$$
?
$$$$
\text { ? }
$$

Habitude courante, notamment chez les princes (les Azande du Nord l'avaient abandonnée).

? Tout le monde (sauf les individus détenteurs d'un fusil).

Quelques individus, mais ni les Avongara,

ni les Ambomu. Étaient cannibales : les

Azande-Bamboy, les Abunge, les Asibali,

les Basiri, les Pambia, les Madi, les

Abarambo.

Les peuples conquis comme les Abarambo

et les Abangbinda, mais non les purs

Azande.

Les Amadi, les Abarambo, les Abangbinda, les Babukur, les Humba, mais non les vrais Azande. Pratique peu courante.
Les prisonniers et les victimes ennemis.

$?$
$?$
$?$

Les prisonniers de guerre, les esclaves,

les gens sans défense.

Les victimes de guerre, les prisonniers exécutés.

Les victimes de guerre, les criminels.

Les victimes de guerre.
Viande.

Viande.

Vengeance, viande, rite religieux, toutes sortes de raisons.

Viande.

Viande.

Viande.

Viande.

Viande ; les testicules servaient à fabriquer une pommade magique. Viande.

Viande. 
Résumons-nous par les conclusions suivantes: 1) Quelques individus pratiquaient le cannibalisme, mais normalement n'étaient mangés que les victimes de guerre et les criminels, et seulement en certaines occasions ; 2) Quelques personnes seulement consommaient de la chair humaine. Aux autres, le cannibalisme semblait horrible, notamment aux Avongara de la maison royale (avec des exceptions possibles, mais assez peu vraisemblables) et aux Ambomu (au moins à la plupart d'entre eux, car il paraît certain que quelques Ambomu ont été cannibales). La majorité des mangeurs d'hommes étaient des étrangers ou des personnes d'origine étrangère diversement assimilés ; 3) La raison en était une prédilection pour la chair humaine, qu'exaspérait parfois la faim.

Je signalerai en outre que sur un tel sujet un excès de prudence ne messied pas à l'égard des témoignages basés sur des ouï-dire. Les Européens et les Arabes semblent avoir manifesté un intérêt morbide pour le problème du cannibalisme et cru la plupart des histoires qu'on leur contait. Un détail humoristique : bien des Azande étaient persuadés que les docteurs britanniques étaient cannibales et qu'ils opéraient pour se procurer de la viande afin de satisfaire leur horrible penchant. Souvenons-nous aussi qu'à une certaine époque on discuta très sérieusement la question suivante: les célèbres Niam-Niam portent-ils des queues ? Quoi qu'il en soit, nous semblons disposer de preuves suffisantes pour formuler nos conclusions.

J'ajouterai seulement que les études, qui essaient d'établir une corrélation entre le cannibalisme et un autre trait social ou culturel, réclament un examen très approfondi et très rigoureux des preuves, comme j'ai tenté d'en effectuer dans cet article, de même qu'une saisie globale de la complexité du problème.

\section{BIBLIOGRAPHIE du chapitre 6}

$\underline{\text { Retour à la table des matières }}$

ANDERSON (capt. B. G.), 1911. Some Tribal Customs in their Relation to Medicine and Morals of the Nyam-Nyam and Gour Peoples inhabiting the Eastern Bahr-elGhazal, Fourth Report of the Wellcome Tropical Research Laboralories, Londres.

ANON, 1896. MM, Nilis and de La Kéthulle on the Borders of Darfur, Geog. J., 7, pp. 428429 (Monthly Record).

ANTINORI (0.), 1868. Viaggi di O. Antinori e C. Piaggia nell'Africa Centrale, Boll. Soc. Geog. Val., 1, pp. 91-165.

AUCAPITAINE (le baron Henri), 1857. Les Yem-Yem, tribu anthropophage de l'Afrique centrale, Nouvelles Annales des Voyages, 6e sér., pp. 3-11. 
BROWNE (W. G.), 1799. Travels in Africa, Egypt and Syria from the years 1792-1798, Londres.

BURROWS (capt. Guy), 1898. The Land of the Pigmies, Londres.

1899. On the Natives of the Upper Welle District of the Belgian Congo, J. Anthrop. Inst., n.s., 1, pp. 35-47.

CALONNE-BEAUFAICT (A. de), 1921. Azande, Bruxelles.

CARMIGNANI (Renzo), 1954. Il Cannibalismo degli Asandè, Rome.

1955. Gli Asandè 0 Niam-Niam e il Problema del loro Cannibalisme, Atti della XLV Riunione della Società Italiana per il Progresso delle Scienze, Rome.

- $\quad$ 1957. Il cannibalismo degli Asandè, Africa, 27, pp. 397-400.

CASATI (Gaetano), 1891. Ten Years in Equatoria, Londres.

COURET (C.-L. du) (Hadji-Abd-el-Hamid-Bey), 1854. Voyage du pays des Niam-Niam, ou Hommes à queue, Paris.

CRAFFEN (Enrico) et COLOMBO (Edoardo), 1906. Les Niam-Niam, Rev. inter. de Sociologie, $\mathrm{n}^{\circ} 11$, pp. 769-800.

CZEKANOWSKI (Jan), 1924. Wissenschaftliche Ergebnisse der deutschen Zentral-AfrikaExpedition 1907-1908, unter Führung Adolf Friedrichs Herzog zu Mecklenburg, Bd 6, 2 : Forschungen im Nil-Kongo-Zwischengebiet, Leipzig.

ELIOT (sir C. N. E.), 1902. Notes of a Journey through Uganda, down the Nile to Gondoroko, juillet 1902, Geog. J., 20, pp. 611-619.

EMIN PASHA, 1888. Emin Pasha in Central Africa, being a Collection of his Letters and Journals (éd. Schweinfurth et al.), Londres.

EVANS-PRITCHARD (E. E.), 1937. Witchcraft, Oracles and Magie among the Azande, Oxford.

- $\quad$ 1955. Review of Renzo Carmignani's, Il Cannibalismo degli Asandè. Africa, 25, pp. 202-203.

- $\quad$ 1956. Cannibalism : a Zande Text, Africa, 26, pp. 73-74.

GESSI PASHA (Romolo), 1892. Seven Years in the Soudan, Londres.

GEYER (Franz Xaver), 1914. Durch Sand, Sumpf und Wald, Fribourg. Sumpf und Wald, Fribourg. 
GIORGETTI (Filiberto), 1957. It cannibalismo dei Niam Niam, Africa, 27, pp. 178-186.

HEUGLIN (M. Th. v.), 1869. Reise in das Gebiet des Weissen Nil and seiner westlichen Zuflüsse in den Jahren 1862-1864, Leipzig et Heidelberg.

HUTEREAU (A.), 1909. Notes sur la vie familiale et juridique de quelques populations du Congo belge, Ann. Mus. du Congo belge, sér. III, Bruxelles.

JUNKER (Wilhelm), 1890, Travels in Central Africa during the Years 1875-1878, Londres. 1891. Travels in Central Africa during the Years 1879-1883, Londres. 1892. Travels in Central Africa during the Years 1882-1886, Londres.

LAGAE (Mgr C. B.), 1926. Les Azande on Niam-Niam, Bruxelles.

LARKEN (Major P. M.), 1927. Impressions of the Azande, Sudan Notes and Records, 10, pp. 85-134.

- («Rukusuru »), 1920. Review in Sudan Notes and Records, 3, pp. 77-78.

LONG (col. C. Chaillé), 1876. Central Africa : Naked Truths of Naked People, Londres.

LOTAR (R. P. L.), 1946. La grande chronique de l'Uele, Bruxelles.

MARNO (Ernst), 1879. Reise in der Egyptlischen Aequatorial-Provinz and in Kordofan in den Jahren 1874-1876, Vienne.

MECKLENBURG-SCHWERIN (Adolf), FRIEDRICH (A. H.), HERZOG (V.), 1913. From the Congo to the Niger and the Nile, Londres.

PASSAMONTI (Eugenio), 1937. La Vita di Carlo Piaggia, Estratto dell' Annuario 1937 del R. Instituto Tecnico Commerciale a Indirizzo Mercantile «Carlo Piaggia», Viareggio.

PETHERICK (John), 1861. Egypt, the Soudan and Central Africa, Edimbourg et Londres.

PA PETHERICK (Mrs), 1869. Travels in Central Africa, Londres.

PIAGGIA (Carlo), 1941. Le Memorie di Carlo Piaggia, G. Alfonso Pellegrinetti (édit.), Florence.

PONCET (Jules), 1863. Le Fleuve Blanc, Paris.

POTAGOS (Dr), 1885. Dix années de voyages, Paris.

REYNOLDS (Harold), 1904. Notes on the Azande Tribe of the Congo, Journal of the Afr. Soc., 11, pp. 238-246. 
SCHWEINFURTH (Georg), 1873. The Heart of Africa (trans. FREWER, E. E.), Londres. 1873 [2]. Das Volk der Monbuttu in Central Afrika, Z. für Ethnol., 5, pp. 1-33.

SELIGMAN (C. G.) et SELIGMAN (B. Z.), 1932. Pagan Tribes of the Nilotic Sudan, Londres.

SPENCE (Basil), 1920. Cannibalism in the Bahr El Ghazal, Sudan Notes and Records, 3, pp. 300-302.

WILSON (Rév. C. T.) et FELKIN (R. W.), 1882. Uganda and the Egyptian Soudan, Londres, 2 vol. 


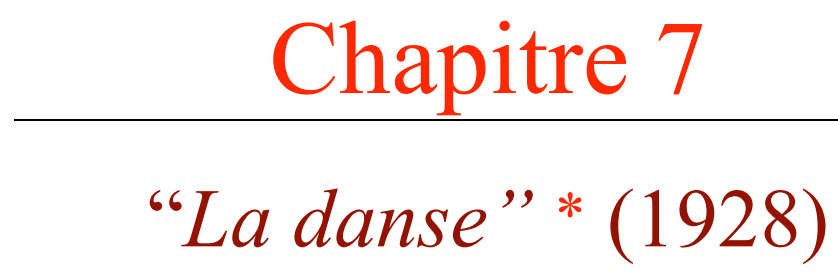

$\underline{\text { Retour à la table des matières }}$

La place habituellement réservée à la danse dans les récits ethnologiques ne correspond nullement à son importance sociale. Souvent saisie comme activité indépendante, elle est dépeinte sans référence au contexte de la vie indigène. De la sorte, se trouvent éliminés les problèmes de composition et d'organisation de la danse, et masquée sa fonction sociologique.

L'analyse sommaire d'une danse africaine prouvera que, par sa structure, elle diffère radicalement de la danse européenne moderne. Elle montrera aussi que, lorsque plusieurs personnes, et, à plus forte raison, plusieurs centaines de personnes, participent à la danse, elle exige une forme stéréotypée, un mode défini d'exécution, des mouvements coordonnés, un leader reconnu, une organisation et une réglementation élaborées. Si l'observateur ne conserve pas à l'esprit ces divers aspects de la danse, il nous en brossera peut-être un tableau intéressant, mais ne nous fournira pas un compte rendu détaillé exploitable pour le plus grand profit du théoricien.

La danse remplit aussi des fonctions physiologiques et psychologiques que seule une description complète et précise peut révéler C'est essentiellement une activité collective et

Paru dans Africa, 1928. Pour une étude complète sur les Azande, cf. LAGAE, Les Azande ou Niam-Niam, 1926, et LARKEN, An Account of the Zande, Sudan Notes and Records, juillet 1926. 
non pas individuelle, aussi nous faut-il l'expliquer en termes de fonction sociale, c'est-à-dire en déterminer la valeur sociale. A ce propos, l'observateur non expérimenté, même lorsque sa vision demeure objective, est si peu habitué à considérer les institutions et les coutumes selon leur valeur fonctionnelle, qu'il néglige souvent de signaler au théoricien l'occasion de la danse, qui seule peut permettre de juger de sa signification.

Dans cet article, j'analyserai brièvement une danse zande en suivant les grandes lignes que je viens d'évoquer. Les Azande sont scindés en trois groupes par les administrations européennes. La plupart d'entre eux vivent dans la partie nord du Congo belge, mais on les rencontre aussi dans les provinces de Bahr-el-Ghazal et de Mongalla au Soudan angloégyptien, et en Oubangui-Chari dans l'Afrique équatoriale française. Les Azande dont je parlerai dans cet article sont ceux du Soudan anglo-égyptien ${ }^{1}$.

Parmi leurs nombreuses danses, certaines sont régionales, d'autres, qui ont cessé d'être exécutées mais dont les anciens se souviennent, peuvent être reconstituées sur demande. Le tamtam ou le xylophone ou un instrument à cordes les accompagnent habituellement. Cependant, quelques-unes ne comportent aucun accompagnement instrumental. Il est des danses propres aux cérémonies de la circoncision, aux différentes sociétés secrètes, aux fils de chefs, aux femmes, aux cérémonies funéraires, aux activités économiques. Comme dans un article aussi court nous ne saurions prétendre en dresser une classification, force nous sera de nous limiter à l'analyse des caractéristiques principales d'un type de danse qu'accompagnent le gong et les tam-tams, la danse de la bière, gbere buda.

\section{La musique}

Retour à la table des matières

Il convient de distinguer dans la gbere buda trois éléments la musique, le chant et le mouvement corporel. Bien qu'on ne puisse concevoir cette danse en l'absence de l'un de ces éléments, leur agencement est difficile à comprendre.

De grands gongs de bois et des tam-tams tendus de peau aux extrémités constituent les instruments de musique. Le grand gong de bois ressemble à un buffle avec ses pattes, sa queue, sa tête et ses cornes. Lorsque le vent souffle à l'intérieur du fût évidé, on jurerait le meuglement d'un buffle. La dimension des gongs varie énormément, certains mesurent de un mètre à un mètre cinquante de haut. Ils sont taillés dans un seul tronc d'arbre et leur fabrication peut demander deux ou trois mois. Sur la partie convexe du tronc, on opère une incision à partir de laquelle on creuse inégalement chaque côté. Le batteur s'assied sur la partie imitant la queue et frappe le gong avec des baguettes dont les extrémités sont entourées de bandes de cuir qui forment une boule de façon à protéger le bois. Il tient une baguette dans

La générosité du gouvernement du Soudan anglo-égyptien ainsi que l'aide financière accordée par la Royal Society et les Laura Spelman Rockfeller Memorial Fund Trustees me permirent de mener à bien mon expédition au Soudan. 
chaque main et frappe le gong de coups brefs ou prolongés. Il peut frapper le gong simultanément avec les deux baguettes ou alternativement. Il règle le volume du son : a) En battant un côté plutôt que l'autre puisqu'ils sont inégalement creusés ; b) En frappant plus ou moins près de la fente ; c) En plaçant une jambe pliée ou allongée sur la fente pour l'ouvrir ou la boucher à volonté ; d) En frappant plus ou moins fort sur le gong.

Le tam-tam, d'environ un mètre, un mètre vingt de haut, se compose d'un tronc de bois creusé et d'une peau d'animal tendue à chaque extrémité. On frappe sur la partie la plus large, le tamtam allant en s'évasant vers le haut. Tenu souvent de biais entre les jambes, le tam-tam est battu du plat de la main par petits coups brefs ou prolongés. L'usage des deux instruments implique une division du travail dans la danse : un homme joue du gong (gugu), l'autre du tam-tam (gaza).

\section{Le chant}

$\underline{\text { Retour à la table des matières }}$

A la musique de ces deux instruments, il faut ajouter celle des voix humaines. Comme je ne disposais pas de magnétophone et que ma connaissance de la musique se borne à des rudiments, je ne parlerai du chant que de façon très générale ${ }^{1}$. Pour notre étude, nous pouvons peut-être distinguer deux ou plutôt trois éléments dans le chant: la mélodie ou succession de notes dans une suite rythmique, le sens ou les valeurs phonétiques prises dans le contexte, le chanteur, non seulement pour le timbre de sa voix, mais aussi pour la variété de ses mouvements.

Je ne m'attarderai pas sur la mélodie. La mélodie zande ne comporte qu'une variété restreinte de mesures. Pour une oreille non entraînée, les mélodies africaines semblent peu variées, ce qui est incontestablement faux : nous jugeons de la diversité des mélodies par comparaison avec les airs européens. Seul le magnétophone peut fournir des données valables. Les mélodies sont éphémères, peu d'entre elles subsistent au-delà d'une saison ou deux, après quoi elles perdent de leur popularité et sombrent dans l'oubli. Ou peut-être, seuls les chants sont-ils transitoires alors que les mélodies survivent dans des combinaisons de thèmes différents.

Tous ces chants possèdent un sens plus ou moins explicite. Dans l'esprit de leur créateur, leur signification va de soi, car ils se rapportent à des personnes et à des événements que lui connaît. Quant à ceux qui les écoutent et les chantent, tout dépend de leurs relations avec les personnes et les faits évoqués. Je me suis rendu compte qu'il n'y avait aucune difficulté à obtenir de l'auteur une explication claire de ses chants. Les autres personnes, bien qu'elles les connaissent et les chantent, n'ont souvent qu'une idée vague de leur signification. L'air et les

Pour de plus amples informations sur la musique négro-africaine, cf. l'article du spécialiste E. M. von HORNBOSTEL paru dans Africa, vol. I, no 1. Des travaux complets sur les chants requièrent l'usage d'un magnétophone. J'ai essayé, lors de mon voyage de retour, de faire noter des mélodies de chanteurs azande par deux musiciens. Ce fut un échec. 
paroles subissent bien des altérations phonétiques et grammaticales qui en modifient le sens. En général, on peut affirmer que c'est la mélodie qui importe et non le sens du texte, ou, comme on dit vulgairement, c'est l'air qui compte et non la chanson ${ }^{1}$.

Cependant, il importe de ne pas oublier quelle arme puissante le chant constitue. On respecte beaucoup un compositeur intelligent et populaire pour son talent et pour son habileté à diffamer ses ennemis. Le chant sert aussi de porte-parole de la loi, en ce sens qu'il exerce une contrainte qui joue le rôle de sanction : il fustige celui qui a offensé l'opinion publique, loue ceux qui se sont distingués, fait l'éloge des princes.

Nous avons considéré la mélodie et le chant, reste le rôle du chanteur. Celui-ci transpose la mélodie dans les gestes de ses mains qu'il tient en l'air comme s'il voulait appeler quelqu'un. A ces gestes, il ajoute une variété de mouvements corporels très différents des mouvements stéréotypés de la danse. Le chant, lors d'une danse, est inconcevable sans des mouvements corporels qui font partie de la mélodie autant que les paroles.

Les chants qui accompagnent la danse de la bière ont une structure spéciale. Comme la plupart des chants africains, ils sont exécutés en alternance par un soliste et un chœur. En fait, dans ces chants, il existe deux sortes de chœur, mais j'y reviendrai plus loin. Tous les chants se composent d'un couplet chanté par le soliste (ndu) et d'un choeur (bangwa). S'il y a plusieurs couplets, le soliste commence le ndu suivant, alors que le chœur termine le bangwa. Ce chevauchement constitue une caractéristique de ces sortes de chants. Voici un exemple qui montrera la division entre choeur et solo $^{2}$ :

ndu wili Bagurunga ke ya mi na di li mi dua kina

(solo) nderugi li angba ti re ni lengo du a du tamamu ka wira kina na Kwamba

bangwa nina ooo ooo ako ooo ooo mi bi pai mbataiyo

(chœur) gbaria tunotuno gbariai ni gbunga ba

ndu Le fils de Bagurunga dit : je l'épouserai, je lui construirai une case d'herbe. Oui, vraiment je l'aime; oh, que je l'aime en vérité.

Elle ressemble beaucoup à la mère Kwamba.

bangwa Mère, ooo ooo, hélas, ooo ooo, je vois quelque chose là-bas. La maison du gouverneur est loin, très loin; la maison du gouverneur est si éloignée.

Les Azande distinguent le sima du solo (ndu) et du chœur (bangwa). J'ai oublié le sens précis du terme sima. Je sais seulement qu'il désigne une partie reprise par le chœur après le soliste. Par exemple, dans le chant ci-dessus, si le soliste chante de wili à Kwamba et que le

E. M. von Hornbostel attira l'attention sur le placage, par les missionnaires, de paroles africaines sur les airs des hymnes européens. Le résultat est peu satisfaisant car les formes musicales européennes ou arabes semblent tout à fait bizarres à une oreille africaine. Les indigènes chantent toujours faux. Il est impossible de traduire les paroles d'un hymne dans une langue africaine sans aboutir à une stupide parodie. Enfin, les mouvements corporels sont exclus du chant des hymnes alors qu'ils représentent un élément essentiel du chant africain.

2 Comme la mélodie n'est pas transcrite, j'ai donné les paroles d'après leur valeur phonétique dans la conversation courante et non pas avec les élisions du chant. 
chœur reprend le tout, ou s'il chante le couplet en plusieurs fois et qu'à chaque fois le chœur répète ce qu'il vient de chanter, on appellera cette partie du chant le sima. Certains chants sont ainsi divisés en trois parties, ndu, sima, bangwa.

Le chant ci-dessus peut aussi s'écrire en vers de telle façon que la fin de chaque ligne soit marquée par une pause. On obtient alors :
wili Bagurunga
ke ya mi na di li
mi dua kina nderugi
li angba ti re ni lengo
du a du tamamu
ka wira kina na Kwamba

Puisque le chant est entrecoupé de pauses, on peut écrire le chœur de semblable manière, afin que les syllabes forment des vers au mètre régulier. Il en va probablement de même pour la plupart des chants.

\section{Les mouvements du corps}

\section{Retour à la table des matières}

Après avoir analysé quelques aspects de la musique et du chant, nous abordons le troisième élément essentiel de la danse, les mouvements du corps. Le Zande danse avec tout son corps. Il ne suit pas simplement le rythme de la musique avec ses pieds, mais, les bras repliés au coude et loin du corps, il agite les mains de haut en bas, secoue la tête d'avant en arrière, s'incline de droite à gauche, hausse et baisse les épaules, fait travailler ses abdominaux. Tous les muscles de son corps semblent en action et l'on croirait que sa peau abrite une multitude de serpents.

Chacun jouit d'une grande liberté pour varier ses mouvements, mais tout le monde suit le rythme, et tous les danseurs exécutent des pas stéréotypés. Cette uniformité s'avère parfois nécessaire comme nous le verrons lors de la description de la danse. Ces pas, après une certaine vogue, sont, comme les chants, remplacés par d'autres. Je ne pense pas qu'il existe de corrélation spécifique entre un type de pas et un type de mélodie. 


\section{Schéma de la danse}

\section{Retour à la table des matières}

De toute évidence, si l'on veut éviter une totale confusion, il convient que la danse suive un modèle défini. La forme déterminée de la danse zande de la bière apparaîtra mieux sur un croquis.

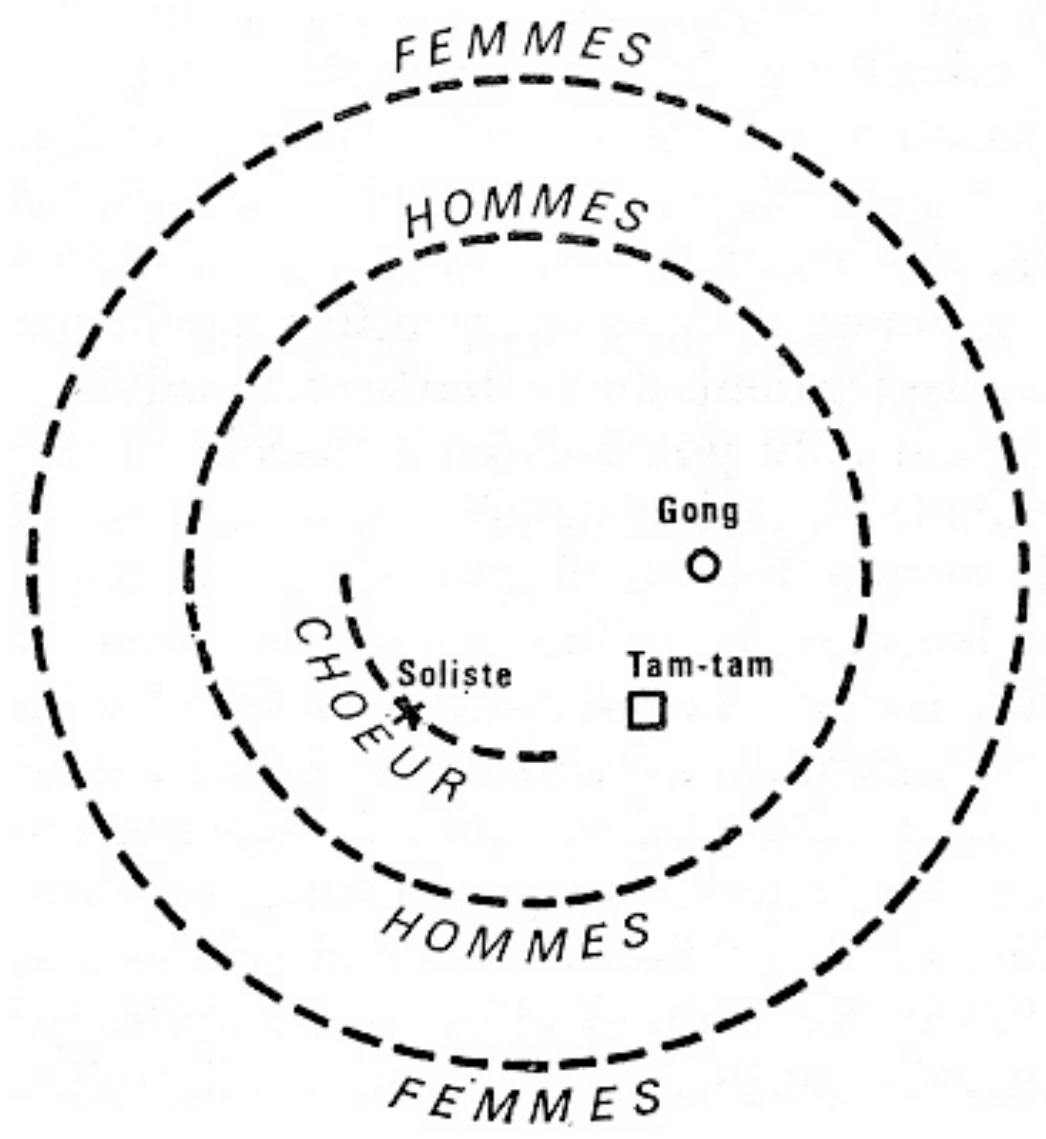

Les hommes, se tenant très proches les uns des autres, face au tam-tam et au gong, forment un cercle fermé. A l'extérieur du cercle, les femmes dansent par groupes de deux, de trois ou davantage. Habituellement les hommes dansent sur place, mais de temps à autre, ils se tournent de côté et à la queue leu leu avancent lentement jusqu'à ce que, bouclant la circonférence, chacun ait retrouvé sa place. Ils se retournent alors face au tamtam. Les femmes, toujours à l'extérieur du cercle des hommes, évoluent en rond à pas lents, les bras autour de la poitrine de celle de leur groupe qui les précède. Une certaine liberté de mouvement leur est accordée. Les jeunes désireux de se manifester quittent le cercle pour 
exécuter, seuls, un pas en direction du tam-tam. Parfois une partie du cercle s'avance vers le tam-tam puis revient à sa place. Les jeunes enfants sautent et courent à leur guise. Lorsque le nombre des participants est trop élevé et qu'un cercle ne suffit pas, on en forme parfois deux ou trois ; les femmes dansent alors souvent entre ces cercles. Lors des danses importantes, on exécute occasionnellement un autre type de danse en même temps que la danse principale de la bière.

\section{Le leadership dans la danse}

\section{$\underline{\text { Retour à la table des matières }}$}

Dans une activité collective, notamment dans la danse, où les règles de la vie sociale se relâchent quelque peu et où chacun se divertit sans frein, il existe toujours un risque de rupture et de désagrégation dû aux tendances égotistes. Certains pourraient outrepasser les limites de la sexualité autorisée ; les garçons, trop désireux de parader devant les filles, pourraient provoquer de mauvaises querelles. Si plusieurs personnes voulaient jouer le rôle de soliste, des confusions et des disputes pourraient s'ensuivre, et les hommes en armes, échauffés par la bière et excités par la danse, risqueraient de se battre ; l'individu victime des quolibets des chanteurs pourrait leur chercher noise.

Une réglementation bien définie et le principe du leadership permettent de surmonter ces risques. Tout d'abord, nous avons remarqué sur le schéma, qu'hommes et femmes sont complètement séparés. Ils ne dansent jamais ensemble. La ségrégation des sexes est une garantie, et, bien que l'amour, comme nous le verrons plus loin, se donne libre cours à l'occasion de ces danses, il n'est jamais audacieux ni provocant. La danse zande de la bière se déroule dans l'ordre et la discipline. Le voyageur, en provenance du Nord ou de l'Est soudanais, qui pénètre en pays zande, cherche toujours à comparer la discipline des Azande avec celles des autres tribus et la danse est un des éléments de sa comparaison. Les danses des Moro, des Mundu, des Baka et des Bongo semblent beaucoup plus violentes et tapageuses que celles des Azande et ne se déroulent jamais avec autant d'ordre et de précision.

Les princes considèrent qu'ils déchoiraient en participant à une danse publique. Néanmoins, leurs fils y assistent souvent et, même s'ils ne sont encore qu'enfants, leur décision, lors d'une dispute ou d'une querelle, fait autorité. Mais la souveraineté de la noblesse n'intervient pas dans la structure même de la danse qui possède son propre leadership. Le conducteur des chants est appelé bayango. Dans une province, il n'existe généralement pas plus de deux ou trois bayango de grande renommée. Le postulant à la charge de bayango doit d'abord apprendre le métier auprès de l'un deux, dont il sera le suali. Les suali accompagnent leur maître lors des danses, ils se tiennent en face ou à côté de lui et soutiennent les choeurs. Les suali ont bientôt appris tout le répertoire de leur maître qu'ils remplacent le cas échéant. La préséance est accordée au principal suali d'un conducteur de 
chants réputé, et, aujourd'hui, il est parfois désigné par des termes arabes utilisés pour les grades de la police gouvernementale : sergent, caporal, soldat de première classe.

Le conducteur des chants ou l'un de ses principaux suali jouit d'une autorité suffisante, en cas de litige, pour attribuer le poste envié de batteur de gong. Des rivalités surgissent toujours lorsqu'il s'agit de jouer du tam-tam lors d'une danse, car le jeune batteur se sait observé par toutes les jeunes filles. Son rôle est le plus désiré après celui de conducteur des chants. S'il n'y a pas de batteur de tam-tam particulièrement qualifié, il n'est pas rare qu'éclate une bagarre. Cependant, en présence d'un joueur de tam-tam renommé (ba ta gaza), nul ne discute ses droits. Le disciple d'un conducteur de chants ou d'un joueur de tam-tam offre, de temps à autre, de petits présents à son maître.

La danse, comme toutes les activités collectives, engendre nécessairement l'apparition d'un leader dont la fonction est d'organiser l'action. Le problème de l'attribution des rôles dans la danse est résolu par l'autorité conférée au statut de conducteur des chants. Si des querelles naissent, le conducteur des chants se pose en arbitre. On lui remet la nourriture et la bière pour qu'il les distribue. On aurait cependant tort de croire que le conducteur des chants dispose d'un large pouvoir ou qu'il appartient à un rang élevé; il ne jouit d'un grand prestige et d'une grande renommée que dans cette activité particulière où il joue un rôle social défini.

Le prestige du leader dépend bien sûr de son adresse à composer et à chanter des chansons, mais, aux yeux des Azande, il doit son talent à la possession du charme adéquat. Quiconque ne posséderait pas ce charme ne pourrait être ni un bon compositeur, ni un bon chanteur. En échange de petits présents, une pointe de lance par exemple, le conducteur des chants fournit des «médicaments » à ses disciples. L'absorption de ces «médicaments »a deux effets. Tout d'abord, elle procure à l'apprenti la confiance qui lui permet de composer et d'exécuter des chansons, et elle lui attire des disciples, suali. Deuxièmement, elle lui sert de diplôme et lui acquiert le droit de prendre la première place dans une danse. Un des hommes à mon service avait l'habitude de se quereller, lorsque nous voyagions dans un district étranger, car il se considérait comme un excellent soliste et essayait de monopoliser ce rôle. Les gens lui demandaient d'où il tenait son charme; en répondant qu'il l'avait reçu d'un conducteur de chants renommé, il désarmait, en quelque sorte, les critiques.

Il en va de même pour le tam-tam. Un jeune homme qui voyage en dehors de son district demande la permission de jouer du tam-tam et explique qu'il est l'élève de tel ou tel batteur de tam-tam connu et qu'il en a reçu un charme.

Lorsque le conducteur de chants donne un charme à l'un de ses disciples, il prend la racine d'une plante et la fait cuire dans l'huile. Tandis qu'il remue les ingrédients, il prononce la formule suivante :

«Tu es le «médicament» de la chanson. Je vais te faire cuire. Ne m'apporte pas la malchance. J'ai chanté bien des chansons. Empêche mes chants de s'affadir. Nous allons chanter avec les conducteurs de chants qui exécutent mes chœurs. Je vais chanter. Les anciens se préparent à danser. Empêche-moi de devenir un mauvais chanteur. En vérité, je continuerai à chanter longtemps. Je vieillirai et je chanterai toujours. Tous mes disciples, tout le monde m'accompagnent lorsque je chante. Tous mes disciples m'offrent des présents. Tu es le «médicament» de la chanson. Si tu es un bon «médicament», tu bous comme l'eau. 
Tu es le «médicament» d'Andegi (conducteur de chants réputé). Je ne t'ai pas volé. J'ai demeuré avec Andegi pendant bien des années et il m'a apprécié. Il m'a conduit à travers le pays et m'a montré où trouver mes «médicaments » et mon charme. Ne dresse pas contre moi les conducteurs de chants. »

Cette traduction de la formule demeure très approximative. Si le conducteur de chants possède un sifflet magique creusé partiellement à une extrémité, avant d'aller à une danse, il s'adresse à son sifflet de la façon suivante :

« Tu es le sifflet de la chanson. Je m'en vais chanter. Le public soutient bien mes chants. Empêche l'assistance de demeurer silencieuse pendant mes chants. Qu'ils ne tombent pas dans le vide. Je chanterai pour les gens, pour les jeunes et les vieilles, pour les anciens et pour tout le monde. Incite-les à venir. Ils vont chanter toutes mes chansons. Ne laisse pas un autre soliste perturber mes chants. Je vais siffler avec toi, sifflet de la chanson. Je ne t'ai pas pris, je t'ai acheté. Ainsi donc je vais siffler. Je siffle, fia. »

Ces deux formules illustrent les rites magiques qui préparent le chant. Il en est d'autres qui servent à assurer le succès d'une chanson. Il existe aussi probablement autant de «médicaments» pour attirer la gloire sur le joueur de tam-tam. Je ne citerai aucune des formules utilisées dans ce dernier cas, car elles ressemblent à celles présentées ci-dessus, seul leur sens diffère.

\section{La fonction sociale de la danse}

Retour à la table des matières

Nous venons d'examiner les éléments de la danse zande, c'est-à-dire la musique, le chant, les mouvements. Nous avons décrit une danse type, montré le besoin d'une organisation et d'un leader, et la manière dont on satisfaisait à ce besoin. Tout au long de notre discussion, nous avons posé des questions concrètes auxquelles nous n'avons souvent pu apporter qu'une réponse incomplète. La formulation de ces questions nous permettra de réexaminer les faits avec une plus grande chance d'obtenir des réponses précises.

Posons-nous d'abord les questions qui doivent toujours prévaloir dans l'esprit de l'ethnographe : quelle valeur attribuer à la danse dans la société primitive ? à quel besoin répond-elle ? quel rôle joue-t-elle? Les récits dont nous disposons sur la danse, chez les peuples primitifs, nous fournissent si peu d'informations sociologiques à son sujet que nous sommes incapables de répondre à ces questions.

Nous nous limiterons à déterminer les éléments de ces problèmes qui doivent susciter l'intérêt de l'observateur, en décrivant plusieurs aspects de la danse zande de la bière. La gbere buda est une activité locale. Ne participent à cette danse que ceux qui habitent à quelques heures de marche de l'endroit où elle a lieu. Les gens se connaissent, ont grandi et 
joué ensemble, travaillé et combattu côte à côte. Beaucoup d'entre eux sont unis par le sang ou par d'autres liens sociaux, tels que le mariage, la consanguinité, la circoncision, des associations animistes, etc. Ils appartiennent tous à un même groupe politique et dépendent d'un même prince. Il importe de se souvenir que la danse est une activité sociale à laquelle prennent part des personnes liées par une expérience commune, basée sur la proximité de l'habitat, et que cette relation se consolide par des sentiments familiaux et d'autres forces socialisantes.

Les gens se rendent à la danse par petits groupes ; amis et parents se rassemblent dans la même section du cercle. Ils viennent de partout pour revoir leurs amis, leurs amants, leurs parents, pour danser, bavarder et s'amuser. Les mères amènent leurs bébés, La danse est la première occasion pour l'individu de faire connaissance avec une société beaucoup plus vaste que le groupe restreint de la famille. Dès que les petits enfants savent marcher, ils courent et sautent, entraînés par le rythme, tout autour des danseurs ou, au centre, près des tam-tams. La danse contribue énormément à élargir le monde de l'enfant et à transformer les sentiments d'exclusivité vis-à-vis des parents, acquis durant la petite enfance.

Lorsqu'ils grandissent, garçons et filles ne manquent jamais une danse. Aux deux sexes, elle offre une possibilité de parader, désir particulièrement vif au moment de la puberté. La danse constitue l'un de ces milieux culturels qui favorisent l'exhibition sexuelle, et encourage la sélection. L'aspect sexuel de la danse n'est pas évident pour un observateur. Garçons et filles viennent danser pour flirter, et le flirt conduit souvent à des rapports sexuels, mais la société insiste pour que tout se passe sans vulgarité ni ostentation. En même temps, elle permet ces rapports sexuels, dans la mesure où ils sont discrets et relativement cachés. Le garçon qui fait ouvertement des avances a une jeune fille se voit réprimandé et injurié. Mais personne n'intervient s'il retient son attention, peut-être par un petit coup de coude, alors qu'elle danse avec ses amis, et quand, s'apercevant que ses sentiments sont payés de retour, il lui dit : «Viens mignonne ! ( mo ye gude). Ils s'en vont tranquillement dans la brousse ou dans une case proche et s'accouplent. Le problème est différent avec les femmes mariées. Les maris, toujours jaloux lorsqu'elles vont danser, les accompagnent généralement. Les hommes craignent de flirter avec les femmes mariées, sous peine de devoir payer des dédommagements élevés aux maris ; autrefois, ils risquaient la mutilation.

La danse appartient donc à ce groupe d'institutions sociales qui permettent à la sexualité de s'exprimer avec discrétion et mesure, qui ont pour fonction de canaliser les forces sexuelles vers des formes d'expression inoffensives pour la société et, simultanément, d'aider le processus de sélection et de protéger les institutions du mariage et de la famille.

Pour les adultes, la danse ne présente pas le même attrait que pour les jeunes. C'est elle qui constitue pour eux, comme pour tous ceux qui se rendent à un bal, la principale attraction. Mais les adultes se laissent moins facilement distraire par le flirt et portent toute leur attention sur le rythme. Habituellement, les personnes âgées ne participent pas à la danse.

Nous n'avons mentionné que quelques éléments importants de la gbere buda, faute de pouvoir aborder ici tous les problèmes intéressants que soulève le spectacle de la danse, ce qui exigerait qu'on la décrive de façon complète et détaillée, dans tous ses aspects. 
Nous voudrions cependant nous poser la question de savoir quelle est la fonction sociale de la danse ? Cette question nous conduira à une réponse générale applicable à toutes les danses, dans toutes les communautés, et distincte de celle applicable aux fonctions spécifiques des danses dans telle communauté particulière et dans telle occasion. Nous ne pensons mieux faire que de résumer l'excellent travail de Radcliffe-Brown sur le sujet. Nous n'entrerons pas dans les détails de son argumentation, mais chacun pourra les lire dans le chapitre $\mathrm{V}$ de The Andaman Islanders ${ }^{1}$.

1. La danse est une activité communautaire à laquelle participe toute la personnalité du danseur : tous ses muscles s'excitent, son attention se concentre, ses sentiments personnels entrent en jeu.

2. Dans la danse, la personnalité tout entière de l'individu se soumet à l'action communautaire. Le rythme et la coutume le contraignent à prendre part à une activité collective où il doit conformer ses actes aux exigences qu'elle pose.

3. L'allégresse, l'énergie et l'amour-propre de chacun des danseurs s'harmonisent avec les sentiments de ses compagnons. De ce concert de sentiments et d'actes individuels découlent une unité et un accord parfaits de la communauté, intensément ressentis par chacun de ses membres.

Dans l'ensemble, ce que nous avions observé à l'occasion de la danse zande de la bière s'accorde avec l'analyse que propose Radcliffe-Brown, de la danse Parmi les Insulaires Andaman.

Chez le danseur, elle entraîne une activité de tout le système musculaire, elle exige l'attention de l'œil et de l'oreille, elle crée un sentiment de vanité. L'expression collective accroît l'intensité de telles expériences. Rythme et coutume poussent certainement l'individu à participer à la danse. Le danseur, obligé de conjuguer ses gestes avec ceux de ses compagnons, tire un certain plaisir de cette coordination obligatoire. La danse tend aussi à augmenter la bienveillance et à créer un sentiment de concorde.

L'analyse de Radcliffe-Brown fournit une base et encourage la recherche. Nous aimerions faire quelques autres observations avant de ratifier sa thèse. Aux réflexions précédentes sur la danse zande de la bière, nous voudrions ajouter quelques suggestions qui modifieraient ces vues en les épurant.

La contrainte qu'exercent le rythme et la coutume n'est pas particulièrement forte chez les Azande. On voit fréquemment des Azande robustes qui ne participent pas à la danse. La coutume ne les y oblige pas, et ils ne semblent pas ressentir une quelconque gêne en ne répondant pas au rythme des tam-tams et de la mélodie. Certaines personnes n'aiment pas la danse et préfèrent rester chez elles au lieu de s'y joindre. Il est vrai qu'il s'agit de personnes parvenues à leur automne, et probablement l'influence du rythme varie-t-elle considérablement avec l'âge : les jeunes enfants y sont plus sensibles que les adultes. Chacun jouit d'une grande liberté par rapport aux autres danseurs qui se comportent souvent

A. B. RADCLIFFE-BROWN, The Andaman Islanders, 1922. 
indépendamment les uns des autres. Là encore, c'est une affaire d'âge : les adultes gardent habituellement leur place à l'intérieur de la danse, alors que les enfants ne tiennent souvent aucun compte de l'organisation d'ensemble. Il faut aussi noter que si l'atmosphère générale de la danse est d'amitié, de tels rassemblements, néanmoins, comportent des risques de rupture pour l'unité et l'harmonie de la cérémonie. Nous avons déjà mentionné certains dangers : les chants diffamatoires, les écarts de conduite sexuelle, l'ivrognerie, les rivalités (l'exhibition devient agressive si on la contrarie), etc., et nous avons essayé de montrer comment les mécanismes sociaux préviennent ces désordres. Les hommes aiment donner libre cours à leurs griefs lors des rassemblements publics. Quiconque a assisté à plusieurs danses de la bière y a vu naître des querelles, et ne souscrirait probablement pas à l'opinion selon laquelle la danse est une activité où règne toujours une parfaite harmonie et où la vanité et lez passions individuelles sont complètement socialisées par les forces contraignantes de la communauté. Radcliffe-Brown n'a pas pris en considération la complexité des motifs de la danse. Nous avons noté quelques points qui nécessiteraient de plus longues observations. Tous les détails sont importants. Il nous faudrait des films présentant les danses dans leur contexte social.

\section{Le rôle de la danse dans les cérémonies religieuses}

\section{$\underline{\text { Retour à la table des matières }}$}

Surtout, il convient de déterminer les occasions de ces danses, de savoir si elles appartiennent à un ensemble de cérémonies, si les danseurs jouent éventuellement un rôle, et lequel, dans l'exécution des rites. Peut-être les danses azande de la bière sont-elles exécutées en diverses occasions, mais en brousse, je ne les ai observées qu'en liaison avec le cycle des cérémonies funéraires ${ }^{1}$.

La danse zande de la bière a lieu lors des festivités en l'honneur des esprits des morts. C'est une obligation sacrée, pour les parents d'un défunt, d'ériger sur sa tombe, entre la première et la cinquième année qui suit sa mort, un monument formé d'un tas de pierres.

Environ un an avant la fête mortuaire, se déroule une cérémonie à caractère économique et religieux, durant laquelle un grand nombre de femmes battent le mil destiné à la fabrication de la bière pour cette occasion. A partir de ce moment, commence ce que nous pouvons appeler le cycle des festivités, qui se poursuit durant un an, jusqu'aux cérémonies finales. Au cours de cette période, on danse, de temps à autre, dans le seul but, semble-t-il, d'indiquer le temps qui reste avant les festivités mortuaires et de rappeler au voisinage que les préparatifs de la fête progressent.

Il existe cependant une exception : cette danse est exécutée lors de la 'Visite des fonctionnaires européens. Parmi les Azande, confinés, par le gouvernement, dans des zones réservées, apparaît une tendance croissante à considérer la danse comme un amusement dénué de tout caractère rituel. 
Le soir, vous êtes assis autour du feu, lorsque vous entendez le roulement lointain des tam-tams, vous en demandez la signification. On vous informe qu'il s'agit d'une fête (pumbo). Dans la fraîcheur de la nuit, vous traversez les hautes herbes humides pour assister à la cérémonie, et vous découvrez, avec déception, que cette petite fête regroupe seulement quarante ou cinquante danseurs et que le maître de la concession n'offre pas de bière. La coutume veut que l'on exécute de semblables danses, de temps à autre, entre le battage du mil et la cérémonie principale en l'honneur des morts.

A cette dernière occasion, la danse revêt une grande importance et plusieurs centaines de personnes peuvent y prendre part. J'ai vu au moins cinq ou six cents personnes y assister, et l'on m'a signalé que des participations supérieures étaient fréquentes. Ces danses mettent le point final au cycle des festivités.

La danse ne doit donc pas être considérée comme simple distraction, mais comme élément d'une activité sociale importante, associé à un cérémonial religieux. Néanmoins, les danseurs ne participent pas aux cérémonies rituelles en l'honneur des esprits des morts. La charge de ces rites intimes revient aux parents du défunt et aux personnes qui entretenaient avec lui d'étroites relations sociales. Les parents ne s'associent pas aux festivités. Leur rôle diffère nettement de celui des amis et voisins venus dans le but de s'amuser. La danse est une importante réjouissance qu'aucun jeune de la région ne voudrait manquer. Ils y accourent avec une humeur de liesse. Mais les activités qui constituent le rituel particulier des esprits et les cérémonies d'échange avec les membres de la belle-famille ne sont pas sans lien avec les activités plus bruyantes et plus profanes de la danse. Même si les émotions des parents du mort et celles des danseurs diffèrent, la danse doit cependant être regardée comme partie intégrante de l'ensemble des cérémonies.

Le roulement des tam-tams attire un grand nombre de voisins à la concession de celui qui se considère comme responsable de l'exécution des devoirs rituels à l'égard des morts. La foule sert de toile de fond à l'accomplissement des rites. Non seulement le grand nombre des participants à la fête flatte celui qui la donne, mais leur présence empreint les cérémonies d'un plus grand sérieux. La foule témoigne que la société reconnaît l'accomplissement des devoirs sacrés dus au mort et des cérémonies d'échange obligatoire entre le maître du festin et sa belle-famille. Une foule imprime au transport banal et déplaisant des pierres sur la tombe, ainsi qu'aux querelles indécentes des échanges sur le nombre de lances et la quantité de bière, un caractère impressionnant et mémorable. Elle transforme les longs préparatifs désagréables de la fête et les obligations fastidieuses des parents en une cérémonie pleine de dignité, en l'honneur des esprits. Telle est, à mon sens, la fonction de la danse, considérée comme partie de l'ensemble d'un cérémonial religieux. 


\section{Chapitre 8 \\ "Signification sociale de la dot en particulier chez les Azande"* (1934)}

\section{Retour à la table des matières}

Dans cet article, j'ai recensé un ensemble de problèmes que j'ai traités succinctement. Dans la mesure où la dot n'exerce une influence que sur les relations matrimoniales, elle peut être considérée comme un moyen de créer de nouveaux liens sociaux durables et fréquents entre des personnes. Elle constitue le processus par lequel se crée le groupe matrimonial, lequel est un groupe réel et non abstrait, dès lors qu'il inclut un certain nombre de personnes qui entretiennent des relations réciproques définies par la coutume. Il ne se compose pas seulement du mari et de sa femme, mais aussi de leurs parents, et se distingue du groupe familial qui naît des relations entre un homme, sa femme et leurs enfants.

La dot a partout une valeur économique. Chez les Azande, elle est constituée par divers outils de fer, lances pour la chasse, houes pour la culture, etc., qui peuvent être techniquement utilisés, ou échangés, soit dans un simple but économique, contre de la bière, de la viande, soit, lors d'une cérémonie rituelle, contre de la bière à l'occasion des fêtes

Résumé d'une communication faite au congrès de la British Association, à Leicester, 1933. Paru dans Man, 1934. 
mortuaires. Chez les Nilotes, la dot est composée de vaches d'une très grande valeur économique, comme productrices de lait entre autres. Du fait que la dot possède une valeur productive et une valeur d'échange, il ne faut pas conclure que sa psychologie correspond à une psychologie de l'achat dans notre propre culture : c'est un argument qu'il suffit d'avancer pour que tout le monde en reconnaisse l'absurdité. Il sera plus profitable de comparer l'aspect sociologique de la dot, en Afrique, avec d'autres techniques économiques dans des régions de culture semblable, et de s'attarder particulièrement sur les sociétés qui ont longuement été en contact avec l'idéologie et le système économique européens, et où la polygynie a disparu, car les relations matrimoniales dépassent celles qui s'expriment en valeur marchande. Les relations entre souverain et citoyen, entre père et fils, entre amoureux et bien-aimée, pour ne citer que trois exemples azande, s'accompagnent aussi de dons ou de versements qui définissent les liens entre les personnes concernées, et leur permettent de prévoir le comportement d'autrui dans certaines situations données, car le paiement engage la conduite sociale. Une étude exhaustive de la dot doit considérer celle-ci comme l'un des nombreux moyens d'établir, de définir, d'exprimer, de conditionner un comportement social, par l'utilisation des richesses sous forme de dons ou de versements.

En général, les personnes qui reçoivent une dot au mariage de leur fille doivent contribuer à doter son frère lors de son mariage. Mais cette obligation ne constitue qu'un aspect mineur de leur droit à recevoir, qui implique d'autres charges bien plus importantes. Le groupe qui reçoit la dot se compose en fait de tous ceux à qui elle est distribuée. Il est utile de connaître la composition de ce groupe avant d'entreprendre une recherche sur le terrain, car elle fournit immédiatement une indication sur l'importance de la structure de parenté dans une société. Ce groupe peut ne comprendre que le père de la jeune fille, comme dans la société zande, ou bien le père et ses parents, ou bien le père, ses parents et ses alliés par le mariage. Il est important de noter que la distribution de la dot s'effectue en vertu des obligations vis-à-vis des parents par le sang et par alliance, et qu'elle est l'expression des relations d'un homme avec ses frères et ses beaux-frères. On la considère généralement, à tort à mon avis, comme étant surtout la reconnaissance d'une parenté entre une jeune fille et ses oncles paternels et maternels.

Si le mariage s'accompagne d'une remise de biens, il engendre aussi un système compliqué d'interrelations qui s'étalent sur une longue période. Chez les Azande, on verse des parties de la dot de temps à autre pendant de nombreuses années avant la création d'un nouveau foyer et on continue à la verser tant que subsiste la nouvelle famille qui a pris naissance par l'établissement de relations matrimoniales. Comme elle est reconnaissance d'un statut social et moyen de conditionner des modes de conduite, elle doit être payée tant que subsiste le statut exprimé dans ces modes de conduite. Le versement renouvelé de la dot peut ne pas apparaître aussi clairement chez les pasteurs, mais les propos des Nuer, par exemple, nous prouvent son existence : tandis que la femme remplit ses devoirs envers la famille de son mari, les vaches donnent des veaux à la famille de la femme, ce qui est très expressément formulé dans les lois du divorce. Les Nuer disent aussi que les parents de la femme ne touchent qu'une partie de la dot au moment de son mariage et qu'ils reçoivent le reste lors du mariage de ses filles. Chez certains peuples, des versements complémentaires sont effectués à la naissance des filles, chez les Fadjelu, par exemple (Whitehaid), et en d'autres occasions, lors d'unions léviratiques. Chez les Azande, ce paiement répété de la dot apparaît plus nettement et nous pouvons le suivre depuis la naissance d'une petite-fille jusqu'à son mariage. Celui-ci n'est d'abord qu'un projet, puis, lorsqu'il prend corps, le paiement de la dot 
commence, et toutes les autres traditions coutumières entre les membres d'un groupe matrimonial acquièrent de l'importance. Si l'un des partenaires meurt, les nombreux liens sociaux tissés n'en sont pas pour autant rompus. Si le mari meurt, sa femme va vivre avec l'un des frères du défunt qui continue d'opérer occasionnellement des versements à sa famille par alliance. Si la femme meurt, ses parents donnent au mari affligé une autre fille, si possible sa sœur ; quant à lui, il continue à verser la dot. Lévirat et sororat sont des moyens de maintenir des relations matrimoniales. Si nous voulons comprendre le rôle de la dot, il nous faut la considérer dans son ensemble et non pas cesser d'en tenir compte, comme on le fait souvent, à partir de la création du nouveau foyer.

Les auteurs négligent fréquemment cet aspect de continuité parce qu'ils envisagent les relations matrimoniales comme les relations qui unissent mari et femme, alors que je considère le groupe matrimonial comme l'ensemble de toutes les personnes qui entretiennent des rapports déterminés en raison du paiement de la dot. Il n'est pas possible de traiter ici tous ces rapports, et je me bornerai à quelques observations sur ceux qui lient le mari et sa belle-famille. L'homme ne paie pas la dot à sa femme, et leurs modèles de comportement l'un envers l'autre se conforment aux normes sociales qui ne sont qu'indirectement liées à l'idéologie de la dot. Il paie la dot à ses parents par alliance. Ce paiement conditionne directement sa conduite envers eux et réciproquement. Leurs relations sont généralement exprimées en référence à la dot (lances de la dot, prestations de travail pour la dot, etc.).

Il n'existe pas de corrélation obligatoire ou constante entre l'idéologie du mariage et la psychologie des personnes en jeu. Lorsqu'un Zande parle du mariage, il s'exprime en termes de dot, une jeune fille dira, par exemple : on a offert des lances à mon père, c'est-à-dire : je suis mariée ; un garçon : je suis l'enfant, des lances, c'est-à-dire, je suis un fils légitime. Il serait naïf de croire que les liens conjugaux ne subsistent que pour des raisons économiques. Pourtant cette supposition sous-tend l'affirmation si souvent exprimée, selon laquelle la fonction de la dot est d'assurer une stabilité au mariage. Le mot «fonction » n'a aucun sens dans ce contexte. Est-il vrai, en outre, que les relations entre mari et femme ne se maintiennent qu'en raison de ce qui est, en fait, un chantage économique ? Aucune preuve n'a pu justifier qu'il existait, comme on le croit parfois, une relation fonctionnelle entre le montant de la dot payée à la famille de la femme et la durée de l'union avec son mari. Personne ne niera que la difficulté de rendre une grande quantité de biens ne motive la pression exercée par les parents d'une femme pour l'inciter à demeurer avec son mari. Mais considérer ce fait comme une explication de la dot revient à déformer les réalités sociales. Il est bon de noter que, chez les Azande, lorsqu'une grande quantité de biens a été donnée, la stabilité de l'union se trouve pratiquement assurée, et les biens traduisent en fait la reconnaissance répétée de cette stabilité. En outre, aussitôt la naissance d'une fille, le divorce n'entraîne plus la restitution de la dot, ce qui infirme l'explication invoquée, laquelle oublie aussi que les relations entre mari et femme ne présentent qu'une importance relative et que la stabilité du mariage repose sur le bon vouloir du beau-père du mari ou de ses beaux-frères. Ajoutons que les désaccords surgissent à propos du versement de la dot, et qu'ils entraînent fréquemment le divorce par voie de conséquence, alors que sont bonnes les relations entre mari et femme. La stabilité de leur union n'est pas si précaire qu'elle dépende de la simple difficulté de restituer la dot, puisque cette difficulté est relativement mineure durant les premières années du mariage. Le mariage et le divorce ne consistent pas seulement à recevoir et à rendre des lances ou du bétail, bien que l'introduction de la monnaie et des échanges européens puissent les influencer dans ce sens. En fait, il appert que la stabilité de la famille 
n'est pas réellement fonction de motifs économiques, mais qu'elle repose sur des normes morales et légales, actuellement en vigueur en pays zande, car, en dépit du paiement de la dot, le divorce est courant. C'est, d'une part, la morale qui censure le divorce, et, d'autre part, la loi qui refuse de reconnaître des motifs de divorce, qui assurent la stabilité de l'union entre mari et femme. Celle-ci découle donc de la contrainte imposée par la loi et la morale, et non d'un chantage économique. Autrefois, les Azande considéraient le mariage comme l'union indissoluble d'un homme et de sa femme, et comme un ensemble de liens permanents entre leurs parents. On n'acceptait le divorce qu'au cas où le mari manquait, de manière flagrante, à ses obligations envers son beau-père ou ses beaux-frères. Torday a constaté le même phénomène chez les Bantu.

Un autre argument est fréquemment avancé : la dot permet aux parents de la fiancée de surmonter leur hostilité vis-à-vis de l'homme qui leur prend leur fille. Aucune preuve ne confirme cette assertion, et on pourrait assurer, au contraire, que le réel danger pour l'union d'un mari et de sa femme n'est pas l'hostilité de la famille de la femme, mais la grande familiarité que des contacts fréquents et les liens de parenté peuvent créer. La dot, un ensemble de tabous - l'évitement de la belle-mère, par exemple -, de modèles de comportement -, la soumission dont la mari doit faire preuve -, sont à l'origine de relations nouvelles qui engendrent l'hostilité et l'opposition, car elles imposent des rapports isolés et distants. Le rôle de la dot est de détourner le ressentiment qui en découle, par des versements complémentaires qui contribuent à l'apaisement. Les époux sont tenus à distance par la famille de la femme, jusqu'à ce que des liens familiaux soient établis, et ce n'est qu'après la naissance des enfants que ces tabous disparaissent.

Laissons de côté les explications psychologiques, car je doute qu'elles puissent beaucoup nous aider, et examinons comment la dot conditionne la conduite sociale. Aussitôt le premier versement de la dot, le comportement des personnes les unes envers les autres se trouve déterminé et fixé d'avance ; il cesse d'être fortuit et imprévisible. En examinant l'intervention répétée de la dot, tout au long de l'histoire des relations matrimoniales, nous pouvons étudier le rôle compliqué qu'elle joue en obligeant les deux groupes à faire face à leurs obligations. Il n'y a pas un versement préliminaire qui entraîne des obligations à venir, mais celles-ci sont déterminées, suivant que les occasions le requièrent, par de nouveaux versements. La dot n'a pas le caractère d'un simple contrat, car la répétition des versements, durant toute la durée du mariage, impose de perpétuels correctifs.

Un autre aspect de la dot doit être mentionné. Dans la mesure OÙ elle constitue un moyen d'établir des relations sociales, il importe peu de l'utiliser de telle ou telle manière pour qu'elle atteigne son but. Mais, en fait, on considère souvent que ceux qui l'ont reçue doivent s'en servir pour obtenir une femme ; dans la mesure où elle est ainsi employée, elle devient le symbole de la valeur de la femme dans le contexte du mariage. Dans toutes les sociétés, le mariage avec certains parents est prohibé ; les plus primitives d'entre elles ont un système social qui permet à l'homme de trouver une compagne en dehors du cercle interdit. Dans bien des sociétés existe le mariage préférentiel : les épouses sont choisies à l'intérieur de la structure de parenté et en vertu de cette structure. Par là même, leur nombre demeure limité ; et les relations sociales ne forment plus que les variantes d'un même type, ou bien se meuvent à l'intérieur d'une même sphère étroite dominée par la structure de parenté. Lorsque les relations matrimoniales se créent hors de la parenté, le nombre des compagnes possibles augmente et de nouvelles relations sociales naissent, indépendantes de la structure de parenté. 
Elles tendent à briser son contrôle exclusif. Des relations sociales nouvelles, notamment des relations politiques, deviennent possibles. La dot et l'exogamie sont alors fonction l'une de l'autre et forment un système interdépendant. Les règles de l'exogamie dépendent du système de mariage en dehors du cercle tabou, et l'efficacité du système de la dot dépend des règles de l'exogamie. La façon la plus directe et la plus simple de se procurer une femme, autrement qu'en vertu du statut de parenté, consiste à échanger une sœur ; mais cette méthode présente des inconvénients, car elle implique que les deux femmes aient à peu près les mêmes qualités. Le mécanisme de la dot permet un choix de femmes beaucoup plus varié que l'échange de la sœur ou le mariage préférentiel. Même dans un mariage préférentiel, on peut payer la dot au sein d'une société où les deux phénomènes coexistent, chez les Hehe (Brown), ce qui pose d'autres problèmes dont j'espère traiter bientôt.

Toutes ces méthodes sont basées sur le principe d'équivalence : en échange d'une femme, on reçoit une femme. L'estimation de la valeur d'une femme par rapport à une certaine forme de biens rend possibles un plus grand choix et une plus grande liberté dans les relations sociales. Pour comprendre le rôle de la dot, il ne nous faut pas considérer un seul groupe matrimonial, mais étudier le transfert de la dot à deux autres groupes. Chez les Azande, le groupe A donne des lances au groupe B en échange d'une femme. Le groupe B transmet les lances reçues au groupe $\mathrm{C}$ pour obtenir une femme. Ni le groupe $\mathrm{A}$, ni le groupe $\mathrm{B}$ n'ont de lances, chacun perd une fille et gagne une femme. Il y a échange indirect, et les lances équivalent à une femme dans le contexte du mariage. Chez les Azande, on ne considère le cycle d'équivalence comme complet qu'à la naissance d'une fille. Quand la femme que le groupe $\mathrm{A}$ a reçue du groupe $\mathrm{B}$ donne le jour à une fille, les personnes du groupe $\mathrm{A}$ savent qu'au mariage de cette enfant elles recevront un nombre de lances équivalent à celui qu'elles avaient donné lors du mariage

de sa mère. Dans le cycle complet, chaque groupe reçoit des femmes en échange de ses filles et des filles en échange de ses lances. La loi zande reconnaît ce cycle, car, lors de la naissance d'une fille, l'obligation de retourner les lances en cas de divorce disparaît. Dans une famille qui ne donne pas naissance à une fille, le garçon peut obliger son oncle maternel à lui procurer, lors de son mariage, les lances qu'il aurait reçues au mariage de sa soeur, s'il en avait eu une. 
La femme dans les sociétés primitives (1963)

Chapitre 9

\section{"La vie quotidienne des Nuer dans les campements de saison sèche"* (1936)}

Retour à la table des matières

Trop souvent, dans les récits anthropologiques, l'attention se concentre sur des concepts sociaux et politiques, tels que la tribu et le clan, et sur des événements particuliers, tels que les cérémonies magiques et religieuses, tandis que l'on néglige le cycle des tâches quotidiennes qui occupent plus régulièrement et plus continûment, on peut même dire plus profondément, les peuples primitifs. Le lecteur peut alors s'imaginer que les activités rituelles tiennent une place plus importante que dans la réalité, et ne pas saisir que c'est justement leur rareté et la rupture qu'elles apportent dans la routine quotidienne qui leur confèrent une signification. Marett a mis en relief cet aspect, en divisant le domaine des activités en un

Article paru dans Custom is King, 1936. J'ai effectué, chez les Nuer, deux expéditions pour le compte du gouvernement du Soudan angleégyptien, et une troisième en tant que chercheur de la fondation Leverhulme. J'ai décrit l'organisation sociale et politique des Nuer dans The Nuer, Tribe and Clan, Sadan Notes and Records, 1933, 1934, 1935, et dans Les Nuer, Gallimard, 1968, traduction française de Louis EVRARD. 
monde prosaïque et un monde d'exception. Pour saisir la valeur du rituel, il faut d'abord connaître le monde prosaïque.

En quelques pages, je vais essayer de décrire la vie quotidienne des campements nuer en saison sèche. Les modes de vie diffèrent légèrement dans les diverses parties du pays nuer. Je parlerai de la vie dans un campement de la tribu Lou à Yakwac sur la rivière Sobat, au milieu et à la fin de la saison sèche 1931.

La vie de campement est monotone et décevante pour l'anthropologue, car rien ne s'y passe. Les Nuer ne sont guère désireux de se battre à cette période de l'année. Les mariages, les cérémonies d'initiation et les cérémonies mortuaires ont habituellement lieu pendant ou à la fin de la saison des pluies. J'ai passé des semaines dans les campements d'éleveurs où se reproduit chaque jour le même cycle d'activités. Décrire une journée, c'est décrire toutes les autres.

La faim est en partie responsable du rythme lent de la vie. Les Nuer Lou cultivent très peu de maiis et de mil, et bien que la disette soit habituellement localisée, dans la plupart des régions, il faut économiser le grain, si l'on ne veut pas qu'il soit épuisé longtemps avant la prochaine récolte. Les Nuer laissent la majeure partie de leur grain dans les villages qu'ils habitent durant la saison des pluies. Ce grain sert de semence, ou de nourriture aux vieillards qui n'ont pu se rendre aux nouveaux pâturages. On consomme, avec ménagement, celui que l'on a emporté. Si les campements ne sont qu'à un jour ou deux de marche du village, les femmes y retournent parfois refaire leurs provisions. Vers la fin de la saison sèche, les vaches, qui ne trouvent pas un pâturage d'assez bonne qualité, maigrissent et ne donnent que peu de lait. Les Nuer qui campent à proximité d'un étang peuvent ajouter du poisson à leur menu, mais, sur la rivière Sobat, nous avions seulement ce que tuaient au javelot, dans les bas-fonds, quelques jeunes garçons, et ce que nous harponnions de notre unique barque. De temps en temps, nous réussissions à abattre quelque bête sauvage, gazelle, cob, tiang, girafe. A part cela, notre approvisionnement en viande se limitait à quelque bœuf ou vache crevée et au produit des saignées pratiquées au cou des vaches, le soir. Les adultes nuer considèrent comme une honte de manger les oiseaux. Parfois, on ramasse des fruits sauvages qui constituent, avec le lait, le menu des Nuer en temps de famine.

La vie de campement est réglée par les exigences du bétail, et les Nuer désignent les phases de la journée par rapport aux tâches pastorales plutôt que par rapport au soleil, ils disent accomplir telle ou telle chose au moment de la traite ou après la conduite des bêtes aux pacages. L'emploi du temps varie peu d'un jour à l'autre. Il est approximativement le suivant

Matin :

- 6.00 Réveil.

- 6.45 Traite des vaches.

- 8.00 Conduite du gros bétail aux pacages et nettoyage du kraal. Repas du matin.

- 8.15 Traite des chèvres, conduites ensuite aux pâturages avec les moutons.

Soir :

- 4.30 Retour des veaux, des moutons et des chèvres au kraal.

- 5.00 Rentrée des vaches qui ont récemment mis bas. 
- 5.30 Retour du gros troupeau et traite des vaches.

- 7.30 Repas du soir.

Tout, le monde s'éveille à l'aube, mais, pendant environ trois quarts d'heure, les hommes, protégés par leurs brise-vent, restent assis près du feu, tandis que les femmes, à l'intérieur de leurs huttes d'herbe en forme de ruche, bavardent, fument et surveillent les bêtes qui dorment encore et ruminent, le museau sur le flanc, ou qui se frottent les unes contre les autres et se lèchent, offrant aux yeux un mélange irrégulier et nuancé de couleurs : fauve, gris, noir, marron, bleu, rouan-noisette.

Les huttes et les brise-vent sont habituellement disposés en demi-cercle, quelquefois en cercle quasi fermé, quelquefois en ligne droite, le dos au vent dominant. Les brise-vent sont de construction simple. Sur les trois quarts d'un cercle, on creuse une rigole étroite. Dans cette rigole, on plante des bottes d'herbe, racines à l'intérieur, que l'on serre les unes contre les autres pour former un écran solide. Au lieu d'herbe, on utilise parfois des tiges de mil. L'extrémité des herbes se courbe sous le vent, mais ne rompt pas. Tout l'espace intérieur est occupé par des foyers de cendres, où les hommes, abrités par le brise-vent, dorment. On ne laisse jamais le feu s'éteindre, et, chaque jour, la hauteur des foyers s'élève par l'accumulation des cendres. Les femmes construisent leurs huttes de la même façon, mais elles rassemblent et attachent ensemble les extrémités des herbes, puis enduisent les parois de bouse de vache. Les Nuer passent plus de la moitié de l'année dans ces abris précaires qui contrastent avec les étables et les solides cases de leurs villages. Parfois, comme à Yakwac, le campement est érigé en bordure d'une rivière dans un village appartenant à des parents. Dans ce cas, les hommes abandonnent à midi leur brise-vent pour se réfugier dans les intérieurs frais des étables, et les femmes passent la nuit dans les cases de terre plutôt que de bâtir des huttes. Mais, même lorsque les pâturages ne manquent pas, comme en certaines régions du pays nuer, les gens, qui ne s'éloignent que très peu de leur village, construisent cependant des brise-vent et dressent un campement. Puisque c'est la vieille coutume de la saison sèche, ils auraient honte de ne pas se déplacer, ne serait-ce que d'une faible distance.

Les bêtes sont attachées devant les brise-vent. Lorsqu'elles sont aux pâturages, le kraal se présente comme un cercle parsemé de piquets et couvert de bouse séchée. Quand elles rentrent le soir, on les attache aux piquets. Les bêtes adultes occupent le centre du kraal, les veaux sont placés près des brise-vent d'où leurs propriétaires peuvent les surveiller, tandis que chèvres et moutons forment de petits groupes entre veaux et vaches. D'énormes bœufs aux cornes immenses, parfois artificiellement déformées, dressent leur masse léthargique et docile au milieu des autres bêtes. Les hommes attachent leur bœuf préféré face à l'entrée de leur brise-vent afin de pouvoir l'observer sans bouger.

Avant et pendant la traite, les jeunes gens paradent en compagnie de leur bœuf favori. Des garçons promènent les bœufs autour du campement et leurs jeunes propriétaires suivent derrière, brandissant leur lance, sautant avec grâce, criant et chantant. C'est une coutume particulière à la saison sèche, qui ne se pratique pas dans les villages durant les pluies. Souvent les jeunes continuent à parader jusqu'à ce que les bêtes partent aux pacages.

La traite commence entre $6 \mathrm{~h} 30$ et $7 \mathrm{~h}$. En cas de brume, elle peut être légèrement retardée. Comme les hommes n'ont pas le droit de traire, ils demeurent derrière les brise-vent, tandis que les femmes et les garçons non initiés s'y emploient. Ce travail prend environ une 
demi-heure, après quoi les bêtes restent attachées un moment. Chaque famille trait ses propres vaches. S'il est des jeunes garçons et filles assez forts, ils accomplissent cette tâche, laissant aux femmes le soin de cuisiner et de s'occuper de leurs bébés. Dans le cas contraire, la femme trait les vaches; elle se charge toujours des bêtes difficiles qui réclament de la poigne et de l'expérience. Les hommes n'interviennent que lorsqu'une vache se montre particulièrement rétive et a besoin d'être fermement tenue.

Entre $7 \mathrm{~h} 30$ et 8 h, on conduit les bêtes aux pacages. Si l'on en détache quelques-unes avant la fin de la traite, elles ne quittent pas le troupeau, mais attendent dans le kraal ou à proximité jusqu'au départ du bétail. Le soleil est alors haut dans le ciel. Lorsqu'un bon pâturage se situe près du campement, les animaux n'y sont pas conduits avant 9 ou $10 \mathrm{~h}$, car ils ont encore toute possibilité de se rassasier avant le coucher du soleil. F. D. Corfield m'a signalé que, chez les Nuer Gaajok et Gaajak de l'Est, dont le pays est moins aride que celui des Nuer Lou, l'on emmenait habituellement les bêtes aux pacages, seulement à cette heure tardive. Personne ne donne l'ordre de détacher le bétail, mais, lorsque toutes les vaches sont traites, quelqu'un commence à détacher les siennes et les autres suivent le mouvement, en terminant par les bœufs. Les bouviers emmènent le troupeau pour la journée. Généralement, plusieurs hommes les accompagnent sur une courte distance, mais il en reste rarement plus de deux ou trois pour garder les bêtes. Chaque famille, à tour de rôle, envoie l'un de ses hommes, et celui qui a gardé le bétail une journée s'arrange avec quelqu'un de la famille voisine pour qu'il s'en occupe le lendemain. Tous les adultes possesseurs d'un brise-vent doivent, chacun leur tour, passer une journée à garder le troupeau.

Après avoir été détachés, certains animaux se dirigent directement vers le point d'eau le plus proche, tandis que les autres attendent d'être rendus aux pâturages pour boire, car, vers midi, les bouviers les conduisent à une mare. Si l'eau est proche, les bêtes y vont d'ellesmêmes ; si elle est éloignée, on les y conduit. On considère la garde du troupeau comme un travail pénible à la charge des jeunes adultes. Au plus chaud de la journée, ils surveillent attentivement le bétail qu'ils peuvent avoir à défendre contre les bêtes sauvages ou les pillards. Souvent, ils rompent la monotonie et la solitude en composant et en chantant des chansons. Avant de quitter le campement, ils prennent un repas frugal, et, à moins de trouver par bonheur des fruits sauvages, ils ne mangent rien jusqu'au coucher du soleil. Malgré la faim qui peut les tenailler, aucun bouvier ne trairait une vache. Ce serait un crime impardonnable.

Dans la région lou, on mène les bêtes paître le long de dépressions marécageuses remplies d'eau au moment des pluies et apparemment à sec pendant la saison sèche. En fait, elles conservent, en profondeur, une humidité suffisante pour que l'herbe y soit verte. L'eau y stagne parfois dans les bas-fonds. Certaines herbes repoussent aussi après le feu de brousse annuel, et conservent une saveur agréable durant la majeure partie de la saison sèche. Pour les trouver, les bouviers conduisent parfois le bétail à plusieurs kilomètres du campement.

Après le départ du gros bétail, et jusqu'à son retour au kraal le soir, les hommes en ont plus ou moins terminé avec leurs tâches routinières, mais les femmes et les jeunes garçons continuent à travailler. Les femmes traient les chèvres et préparent le repas du matin. Les garçons étendent le fumier qui s'est accumulé pendant la nuit, afin qu'il sèche au soleil et serve de combustible le soir. Ils aplanissent aussi la partie du kraal qu'occupent les animaux, en répandant sur le sol des cendres, de l'urine et de la bouse. Ils aident à traire les chèvres et à 
attacher les jeunes veaux à l'ombre. Ils emmènent paître les veaux plus âgés, les moutons et les chèvres.

Les garçons d'un certain âge s'occupent des veaux qui quittent le kraal peu après le gros troupeau. Ils les mènent dans une direction opposée à celle prise par ce dernier, afin que les veaux ne puissent téter leur mère. Les plus jeunes garçons gardent les chèvres et les moutons à proximité du kraal, et passent la majeure partie de la journée à jouer. Si un jeune garçon a très faim, il attrape une chèvre par les cornes et trait un peu de lait dans sa main, ou bien tète à même les trayons de l'animal. On laisse faire ces enfants, car, jeunes, ils ont besoin d'être bien nourris pour devenir des adultes vigoureux.

Tous les hommes adultes ne demeurent pas oisifs, tandis que les femmes et les garçons traient les vaches et nettoient le kraal. La plupart du temps, par groupes de deux ou trois, en compagnie de leurs chiens, ils quittent le campement à l'aube, à la recherche d'une gazelle ou de petit gibier, tandis que certains partent vérifier leurs pièges à girafes. Tous rentrent une fois la traite terminée. Après le départ du bétail, les hommes flânent pendant quelques heures, généralement ils fabriquent des outils, des ornements, ou bien se coiffent.

Vers 10 h, la plupart des hommes dorment derrière leurs brise-vent, sous les arbres, ou dans les étables si le campement est installé près d'un village. A midi, l'activité masculine est réduite à néant, à moins que quelque gibier ne soit en vue. Les hommes saisissent alors leurs lances et, avec leurs chiens, gagnent l'animal de vitesse, l'encerclent, l'acculent. Après les premières pluies, tous les hommes valides quittent le camp à la recherche des traces de girafe. S'ils débusquent des animaux, ils les poursuivent jusqu'à épuisement, les rejoignent et les tuent d'un coup de lance. Les Nuer chassent parfois la girafe pendant une journée entière ; leur endurance est incroyable et particulièrement remarquable quand on sait qu'à cette période de l'année le manque de nourriture les affaiblit. En pleine saison sèche, il est inutile de poursuivre la girafe, trop rapide sur un sol ferme. Tandis que les hommes dorment, les femmes vaquent à leurs besognes quotidiennes. Elles battent le lait de la veille au soir et placent à l'ombre de leurs huttes, jusqu'à la fin de l'après-midi, la portion de la traite du matin destinée à la fabrication du beurre

Vers midi, jeunes gens et jeunes filles vont se baigner à la rivière ou à l'étang, dans un endroit sablonneux et limpide, Ils s'assoient dans l'eau, s'éclaboussent, flirtent et jouent ensemble. Vers 2 h 30, ils rejoignent le campement. Les jeunes filles s'occupent alors du lait, pilent et moulent le mil pour le repas du soir. Les hommes retournent le fumier qui sèche, avec de longs bâtons, puis, n'ayant rien d'autre à faire, ils réintègrent leurs brise-vent.

Vers 4 fi 30, on va chercher les jeunes veaux à l'endroit ombragé où ils ont passé la journée, on les attache dans le kraal et on les force à boire de l'eau. Les garçons reviennent avec les autres veaux, les chèvres, les moutons. Chaque famille attache ses propres bêtes. Un ou deux hommes se portent alors généralement à la rencontre du gros troupeau. D'autres rassemblent, dans des peaux, le fumier séché, précédemment entassé par les enfants, et chaque propriétaire d'un brise-vent prépare le feu dans son foyer. Il n'est pas nécessaire de le réallumer, car toute la journée il a couvé ; aussi suffit-il d'enlever les cendres qui le recouvrent et d'amonceler le nouveau combustible sur les braises découvertes. D'épais nuages de fumée s'élèvent alors et enveloppent le campement. 
Vers $5 \mathrm{~h}$, les vaches qui ont récemment mis bas reviennent en courant, annonçant l'approche du troupeau qui arrive un quart d'heure ou une demi-heure plus tard. Les hommes adultes, dans chaque famille, attachent le bétail, tandis que femmes et garçons se mettent immédiatement à la traite qui se termine au coucher du soleil. Le soir, on ne trait pas les chèvres qui n'ont pas été séparées de leurs chevreaux durant le jour. Les femmes préparent ensuite le repas du soir et s'occupent du lait. Les hommes, près de leur brise-vent, attendent le repas du soir que l'on sert entre $7 \mathrm{~h}$ et $8 \mathrm{~h}$. Les Nuer mangent deux fois par jour; le matin et le soir. Leur repas habituel se compose d'une bouillie de mil, semblable à du porridge, arrosée soit de lait et de beurre, soit de l'un ou de l'autre. Au repas du matin, on mange les restes du soir réchauffés ou arrosés de lait chaud.

Dans les campements importants, on danse souvent le soir. Jeunes gens et jeunes filles viennent alors des campements voisins. Dans les campements plus restreints, les jeunes gens ne se soucient guère de danser avec les rares jeunes filles, leurs cousines pour la plupart. Les habitants des campements bavardent alors près de leurs brise-vent ou sur le seuil de leurs buttes, pendant une heure ou deux après le repas du soir, puis se couchent. Ils parlent parfois jusqu'à minuit, et il arrive qu'un homme particulièrement gai se promène au milieu du troupeau en sonnant une clochette et en chantant. Lorsqu'un animal s'est égaré chacun en éprouve de la tristesse, et les propriétaires passent une partie de la nuit à le chercher dans la brousse.

Si les anciens préfèrent la saison des pluies avec son abondance de nourriture et de bière, les jeunes, dans l'ensemble, aiment mieux la saison sèche. L'absence de moustiques, de boue, d'humidité, accroît le plaisir de dormir en plein air, qui constitue l'un des attraits de la vie de campement. Mais la possibilité de vivre constamment avec ses camarades, de bavarder avec d'autres jeunes filles que ses cousines, représente pour l'homme l'avantage essentiel. Donc, lorsque je parle de la monotonie de la vie de campement, il ne s'agit pas d'une impression ressentie par les Nuer, même si les imprévus sont rares dans la succession quotidienne des activités.

Les jours se ressemblent dans les campements nuer. On mesure le temps à la croissance des veaux qui naissent. A cette période de l'année, la vie s'écoule sans beaucoup de variantes. Lorsque la rivière baisse, on peut pêcher dans les marigots, les bas-fonds et se baigner dans son lit sablonneux. Puis les marigots se dessèchent. Quand la rivière monte, les poissons nagent entre les herbes et les joncs, et, dans certains cours d'eau, de la rive, on les tue facilement à la lance. Trop abruptes, les rives de la Sobat ne le permettent pas. Il n'est plus alors que quelques baignades hâtives en raison des eaux trop profondes.

On change de pâturages de temps à autre, quand l'herbe s'épuise et que cours d'eau et marigots se dessèchent. A Yakwac, à la fin de la saison sèche, la pâture ne devenait possible que là où les herbes des marais conservaient encore l'humidité, à proximité des bas-fonds. En pays lou, c'est souvent un problème d'assurer la survie des bêtes jusqu'à la chute des premières pluies et à l'apparition de nouvelles herbes. Les premières pluies tombent habituellement fin avril, début mai, et partout, pâturages et brousse reverdissent, tandis que la terre, cuite par le soleil, se transforme en bourbier. Les marigots desséchés deviennent des cours d'eau qui s'écoulent vers l'intérieur des terres et le soleil brûlant se voile de nuages. 
La migration des bêtes sauvages loin des rives de la Sobat annonçait les premières chutes de pluie. Les girafes s'en allaient vers les pâturages intérieurs où des mares d'eau de pluie leur permettaient de se désaltérer sans craindre les chasseurs. D'autres animaux se déplaçaient également vers le sud, même les gazelles. Cependant, sur la rive nord, les buffles venaient encore à la rivière où les Nuer affamés les attaquaient. Hommes et bêtes suivent le même rythme des saisons, et lorsque étangs et marigots constituent les seuls points d'eau, il leur faut boire aux mêmes endroits. A la chute des pluies, tous se dirigent vers de nouveaux pâturages.

En mai, non seulement le ciel nuageux et les migrations du gibier, mais les activités ellesmêmes du campement, annoncent le retour au village. Les femmes partent les premières, et on les rencontre, le long des sentiers, qui emportent leur matériel dans des paniers de jonc rectangulaires. De bonne heure le matin, les jeunes gens quittent le campement afin de couper, pour attacher les bêtes, des pieux, qu'ils passent une bonne partie de la journée à tailler. Ils tressent aussi de nouvelles cordes. Comme le temps est plus frais, le besoin de sommeil ne se fait pas autant sentir à midi. Tout le monde s'agite et souhaite rejoindre les villages. Au début de juin, les campements les plus proches du nôtre, ceux de Dengatwor et de Wecgin se dispersent, et nos compagnons de Yakwac partent quelques jours plus tard.

La saison sèche (mai) est terminée, la saison des pluies (tot) a commencé. Les campements (wec) se dispersent et les villages (cieng) retrouvent leurs habitants. C'est la fin des longues journées oisives; hommes et femmes s'occupent maintenant aux durs travaux des champs. Chaque année, les mêmes migrations saisonnières, des villages aux campements, et des campements aux villages, se reproduisent. 


\section{Chapitre 10}

\section{"Les noms de personnes chez les Nuer" * (1948)}

$\underline{\text { Retour à la table des matières }}$

Cet article étudie, chez les Nuer Nilotes du Soudan anglo-égyptien, les différentes manières de s'adresser à une personne, exception faite des termes de parenté. Les titres de politesse que nous allons mentionner s'emploient tous comme salutations. Ils peuvent aussi être utilisés pour attirer l'attention de quelqu'un ou au cours d'une conversation, bien que les Nuer ne répètent pas habituellement, dans la conversation courante, le nom d'une personne, ni ses autres titres de politesse.

L'étude des noms et titres de politesse revêt une certaine importance, car ils symbolisent, comme nous le verrons plus loin, la position sociale d'un homme par rapport à ceux qui l'entourent, si bien que l'emploi de telle ou telle formule permet de connaître immédiatement le statut des personnes en présence. Comme les termes de parenté, ces manières de se désigner ou de s'interpeller mettent en relief les rapports sociaux, conditionnent la réponse à la relation particulière impliquée dans la salutation, et remplacent souvent les termes de parenté, notamment entre proches parents.

Article paru dans The Uganda Journal, 1948. 
Chaque Nuer possède un nom particulier ou nom de naissance, cotdu pany, son vrai nom, qui lui est attribué, sans cérémonie, peu après sa naissance, par son père et sa mère, parfois à la suggestion d'un parent, mais sur décision finale du père. Sa vie durant, il conserve ce nom qui se répercute à travers la façon de s'adresser aux enfants, en particulier aux garçons, souvent désignés ou interpellés comme «fils d'un tel». En définitive, ce nom devient un point de repère dans la structure lignagère.

Dans le village de son père, un garçon est appelé par son nom de naissance. Lorsqu'il devient adulte, les membres de sa famille, ses parents proches du côté paternel, les amis de sa classe d'âge, ainsi que ceux de la classe qui le précède et de la classe qui le suit immédiatement, continuent d'utiliser ce nom. L'emploi de ce nom par un individu beaucoup plus jeune que l'homme auquel il s'adresse pourrait être considéré comme un excès de familiarité. Quiconque s'adresse à un homme plus âgé utilise le terme gwa, "père », qu'à leur tour les plus jeunes emploient également à son endroit. Lorsqu'on s'adresse à des femmes mariées ayant des enfants, ou qu'on les désigne, l'usage du nom de naissance est rare, bien qu'il ne soit en rien inconvenant. Par respect, on utilise généralement la forme technonymique. Pour parler aux jeunes filles, on emploie leur nom de naissance. Quant aux enfants, jusqu'à ce qu'ils connaissent les usages, ils appellent tous les gens, y compris leurs parents et les anciens, par leur nom de naissance.

IL C. Jackson ${ }^{1}$ nous cite un certain nombre de ces noms. Ils se rapportent en général ou bien à des événements qui se passèrent au moment de la naissance, ou bien au lieu de la naissance. Chacun connaît habituellement l'origine de son nom. En voici quelques exemples : Reath, sécheresse ; Nhial, pluie ; Nyuot, violent orage ; Pet, nom de mois ; Ngaluaak, nya, préfixe féminin ; Luaak, étable; (Nya)wec, campement; (Nya)puol, mare ; (Nya)pun, riz sauvage ; Duob, sentier ; Ruei, crachat (quand une femme n'avait pas eu d'enfant, les parents de son mari crachaient dans une gourde, puis on lui frottait le ventre avec ces crachats en priant pour sa fécondité) ; Deng (un prophète habité par l'esprit Deng exécutait un semblable rite) (Nya)gaak, querelle (l'enfant naquit au moment d'une querelle) Met, tromper (lorsque le père de l'enfant courtisait sa mère il se vantait du nombre de bêtes qu'il offrirait en dot, mais, quand vint le moment de les donner, il commença à dire que la vache noire était morte, que la vache blanche avait été mangée par un lion, etc.) ; les noms de la catégorie appelée juoli sont donnés aux enfants dont les frères et sœurs aînés sont morts, par exemple : Bath, perdu ; Cuol, compensation ; Bilieu, tu mourras ; Mun, terre ; Lut, pleur; Gac, découragement ; (Nya)mer, larme.

Les grands-parents maternels d'un enfant lui attribuent souvent un second nom. On s'attend à ce qu'ils élèvent le premier enfant de leur fille, et c'est une habitude courante de sevrer un enfant en le laissant quelques mois chez sa grand-mère maternelle. Lorsqu'une personne se trouve dans le pays de sa mère, on l'appelle, comme le font d'ailleurs habituellement ses parents maternels, par son second nom. C'est pourquoi hommes et femmes ont généralement deux noms, l'un employé par la famille paternelle, l'autre par la famille maternelle. Leur signification se ressemble fréquemment: Lul, pleur; Wia, cri

Dans Sudan Notes and Records, vol. VI, 1923, pp. 142 et 171, JACKSON prétend que les sobriquets ne sont pas rares, mais je ne me souviens pas avoir entendu aucun nom qui méritât cette appellation. 
d'avertissement ; Mun, terre; Tiop, mélange de terre, de fumier et de cendres ; Nhial, pluie (ciel) ; Deng, esprit d'en-haut.

Dans la généalogie d'une famille, on retrouve parfois les mêmes noms. Je n'ai rencontré qu'un seul homme qui portât le nom de son père probablement décédé au moment de sa naissance. Un homme reçoit occasionnellement le nom de son grand-père ou de son arrièregrand-père paternel, de sorte que l'on se remémore le nom de l'ancêtre dans la conversation courante. Dans ce cas, les Nuer disent du garçon « qu'il se tient sur la tête de son grand-père (ou de son arrière-grand-père) », ce cung ni wic gwandongde. Peut-être donne-t-on aussi aux femmes le nom de leur grand-mère ou de leur arrière-grand-mère, mais les informations à ce sujet me manquent.

Les jumeaux portent des noms particuliers qui permettent de les reconnaître immédiatement. L'aîné s'appelle généralement Both (Nyaboth), « celui qui vient d'abord », et le deuxième Duoth (Ngaduoth), « celui qui suit ». On les nomme aussi Gwong, «pintade », Ngec, « francolin », ou simplement Dit (Nyadiet), « oiseau », car les Nuer prétendent que les jumeaux sont des oiseaux. Les enfants qui naissent d'une même mère, après des jumeaux, reçoivent aussi des noms stéréotypés : celui qui vient immédiatement après s'appelle Bol (Ngibuol) ou Bicok et le suivant Tot (Nyatot) ou Cuit.

Chaque enfant hérite d'un col paak, nom propre à son clan et titre d'honneur. Bien qu'on puisse appeler un homme ou une femme par ce nom, en fait, seules les femmes l'utilisent parfois, principalement à l'occasion de cérémonies. Lorsque les femmes s'adressent ainsi aux hommes, c'est généralement en des occasions solennelles, par exemple au retour d'une longue absence, aux mariages et aux cérémonies d'initiation. Ainsi, lorsqu'un jeune homme revient d'un long voyage, il est bien que sa mère et les femmes les plus âgées de la maison s'adressent à lui, en réponse à son salut, par le nom honorifique de son clan, tandis que les jeunes femmes l'appelleront par son «nom de boeuf» (cf. ci-dessous). Les femmes utilisent plus souvent leur nom de clan entre elles, bien qu'il demeure d'un emploi solennel. Pour une belle-mère s'adressant à la femme de son fils, il constitue un titre normal de politesse. J'ai entendu des femmes donner ce nom à leurs petits-enfants, lorsqu'elles étaient très contentes d'eux. J'ai recensé autre part ${ }^{1}$ un certain nombre de ces noms.

Dans le pays de son père, les femmes s'adressent à un individu par le nom honorifique de son clan paternel, dans le pays de sa mère, elles emploient celui du clan maternel. Ainsi, un homme du clan Gaatgankiir né d'une femme du clan Gaatnaca sera appelé, dans la région Jikany où domine le clan de son père, gat (fils de) you et dans la région Lou où domine le clan de sa mère, gat (fils de) nya (fille de) gun. En pays Jikany, on appellera une femme nya you, et en pays Lou, nya nya gun.

Les noms particuliers sont donnés peu après la naissance. Quant aux noms honorifiques du clan, ils sont hérités des parents. Plus tard, hommes et femmes reçoivent d'autres noms. Les garçons sont souvent désignés, par leurs camarades, par référence aux veaux mâles des vaches qu'ils traient. Ce nom, tu en présence des anciens, n'est d'ailleurs jamais utilisé par eux. Au moment de l'initiation, un garçon reçoit, de son père ou de son oncle paternel, un bœuf, ou un jeune taureau qu'il castre plus tard, et qu'il appelle thak gareda, « bœuf de mon

Sudan Notes and Records, vol. XVII, 1934, pp. 28-29. 
initiation ». L'animal devient son favori. Le garçon prend alors son col thak, son «nom de bœuf », évoquant la répartition des taches, la forme des cornes, ou quelque autre particularité de l'animal. S'il acquiert un nouveau bœuf qu'il préfère, ou lorsque meurt le premier, il peut s'attribuer un autre nom qui remplacera l'ancien ou s'y accolera. La plupart des hommes semblent conserver leur vie durant le «nom de bœuf» de leur initiation, par lequel s'adressent habituellement à eux les gens de leur famille, à moins qu'ils ne désirent être appelés par un «nom de bœuf » de leur choix. Cependant, si l'on remarque qu'un garçon s'occupe particulièrement d'un beau bœuf de son troupeau, par exemple qu'il lui frotte des cendres sur le dos, que le matin il chante et parade derrière lui dans les campements de la saison sèche, on lui attribue le nom de ce boeuf. Au début, seuls ses compagnons de même classe d'âge connaissent le «nom de bœuf» d'un garçon initié, mais bientôt les vieux l'apprennent dans les campements qui ont lieu après l'initiation. Dans ces campements, le jeune s'exhibe souvent avec son bœuf et proclame son nouveau nom à qui veut l'entendre ; ses compagnons d'âge l'emploient aussi en présence des anciens. Un homme crie le nom qu'il a emprunté à son bœuf préféré (thak moc) lorsque, d'un coup de lance, il blesse ou un ennemi à la guerre, ou un animal à la chasse, ou un poisson à la pêche. Les jeunes gens du même âge ou d'âges voisins s'apostrophent aussi par leur «nom de bœuf », auquel ils ajoutent des fioritures, lors des danses qui suivent un duel à coups de bâtons ; les jeunes gens se tiennent sur deux files, l'une en face de l'autre, et se renvoient mutuellement leurs « noms de bœuf » avant d'effectuer ensemble un saut spectaculaire.

Les compagnons d'âge d'un homme s'adressent à lui, notamment lorsqu'ils le saluent, par son «nom de bœuf» qu'ils ont le privilège de pouvoir utiliser. Néanmoins, des hommes d'âge sensiblement égal peuvent aussi se servir, entre eux, de leurs «noms de bœuf », s'ils appartiennent à des classes d'âge immédiatement voisines. Il serait inconvenant d'appeler par son « nom de bœuf » un homme beaucoup plus âgé que soi. Les plus jeunes lui disent gwa, «père », et lui les appelle gatda, «mon fils ». Si un étranger désire saluer un homme de son âge par son «nom de bœuf » et qu'il l'ignore, il peut employer l'un des «noms de bœuf » les plus courants, Kurjok, par exemple. A la mort d'un homme, on oublie généralement son «nom de bœuf ». C'est habituellement son nom de naissance qui passe à la postérité. J'ai inséré dans mon ouvrage sur les Nuer ${ }^{1}$ un paragraphe sur la formation des «noms de bœuf ».

Les jeunes filles prennent aussi des «noms de bœuf », ceux des veaux mâles des vaches qu'elles traient, et elles emploient ces noms entre elles. Les jeunes garçons appellent parfois les jeunes filles avec lesquelles ils flirtent par leur «nom de bœuf » et réciproquement, mais jamais en publie. Un homme s'adresse rarement à ses sœurs ou aux autres jeunes filles de la maison par ce nom, à moins de vouloir leur manifester sa satisfaction. Les «noms de bœuf » des jeunes filles ne sont donc guère utilisés qu'entre elles et ne sont que rarement entendus, sauf lors des danses où elles s'interpellent par ces noms en leur apposant des qualifications fantaisistes : toar mathda, filtreuse de bière, mon amie ; gwith mathda, fierté, mon amie ; thiau, bracelets (pour une jeune fille qui en porte). Les femmes mariées prennent les noms de leur vache préférée qu'elles utilisent entre elles. Jamais les hommes ne s'adressent de la sorte aux femmes.

Nous avons noté que, lors des danses, les hommes et les jeunes filles s'interpellent respectivement entre eux par leurs «noms de bœuf» auxquels ils ajoutent de nombreuses

The Nuer, 1940, p. 46; trad. franç., 1968, pp. 64-65. 
fioritures et des qualifications fantaisistes. Fioritures et qualifications sont des noms de danse. Le nom de danse (col buol) précède le «nom de bœuf » qui l'accompagne pratiquement toujours. A cette occasion, les femmes mariées ajoutent aussi des noms de danse à leurs «noms de vache ». Aucune relation de sens ne semble habituellement exister entre les noms de danse et les noms de bétail. Les noms de danse sont parfois des surnoms (usités seulement à l'occasion des danses) donnés par des amis, mais plus fréquemment des noms traditionnels choisis pour de simples raisons d'euphonie, par exemple, pour les hommes, Yar (boudeur) Joklieth («nom de bœuf»), Rang (bonne lance) Boipar «( nom de boeuf »); pour les jeunes filles, Pun (riz sauvage) Rolnyang («nom de bœuf»), Keat (buisson bordant la rivière) Nyinyar («nom de bœuf»); pour les femmes mariées, Biet (silencieuse) Wityan («nom de vache »), Rier (moelle blanche des roseaux) Kuaar Nyang (« nom de vache »).

On appelle souvent un homme d'un certain âge gat, «fils de » suivi du nom de son père. C'est la meilleure manière de saluer, pour les jeunes s'adressant à leurs aînés lorsqu'ils n'utilisent pas gwa, "père », de même que pour quiconque veut témoigner du respect envers quelqu'un, parent ou non. Une telle salutation s'emploie particulièrement lorsqu'un individu est trop jeune pour qu'on l'appelle gwa, et trop vieux pour qu'on utilise son « nom de bœuf ». Rien n'empêche cependant un ancien d'adresser, s'il le désire, cette salutation flatteuse à un jeune homme, comme il le fait parfois. Lorsqu'on parle de quelqu'un, on peut accoler directement à son nom celui de son père : tandis que l'on saluera Wia, fils de Cam en utilisant gat (fils de) Cam, on le désignera, mais peu couramment, comme Wia Cam. Il est aussi possible de s'adresser à quelqu'un par le « nom de bœuf » de son père qui remplace alors son nom de naissance.

Comme je l'ai expliqué ailleurs ${ }^{1}$, un homme (ou une femme), suivant son lieu d'habitation, la qualité de son interlocuteur et l'occasion de leur conversation, peut être désigné par le nom de son pater, de son genitor, de son père nourricier, ou de son père adoptif dans les mariages avec l'esprit d'un mort, et dans les mariages léviratiques, bien que ce soit le nom du pater qui, dit-on, survive dans la structure lignagère. Ainsi, un garçon qui vit avec sa mère et l'amant de celle-ci dont il est le fils, peut être appelé par le nom de son genitor et père nourricier dans le pays de celui-ci, mais, dans le pays de son pater, il portera le nom de ce dernier. Un garçon né dans un mariage avec l'esprit d'un mort est désigné par le nom de son père adoptif, sauf lors des cérémonies où le nom de son pater permet d'indiquer son rang dans la structure lignagère.

On s'adresse plus rarement aux jeunes filles par le nom de leur père. Cette manière de saluer les femmes est réservée à certaines situations et a valeur de compliment. Par exemple, il serait correct, bien que non obligatoire, qu'un homme, s'il n'emploie pas les termes de parenté, appelle l'une des femmes de son père, autre que sa mère, nya, « fille de », suivi du « nom de bœuf » du père de cette femme.

Si un homme vit chez ses parents maternels ou s'il leur rend visite, ceux-ci le saluent du nom de sa mère et non pas de celui de son père, à moins qu'ils n'utilisent les deux

Some Aspects of Marriage and The Family among the Nuer, RhodesLivingstone Papers, ${ }^{\circ}$ 11, 1945, pp. $39-42$. 
simultanément. Dans les mêmes circonstances, on s'adresse aux femmes par le nom de leur mère.

L'usage technonymique, désignation d'un homme par le nom de son fils aîné - gwan (père de) un tel - n'est pas aussi courant que l'usage patronymique, désignation d'un homme par le nom de son père. Il est pourtant aussi flatteur, et il constitue la manière correcte de saluer une personne de la belle-famille ; les beaux-parents notamment s'adressent à leur gendre de cette façon. On évite généralement l'emploi des noms de naissance lorsqu'on parle à sa bellefamille, surtout au cours de la période qui suit immédiatement le mariage. J'ai ouï dire que certaines personnes qui se considéraient comme encore jeunes ne tenaient pas à ce qu'on les appelle du nom de leur fils aîné pour ne pas paraître âgées.

La formule parallèle - man (mère de) un tel - constitue la manière normale de s'adresser à une femme mariée, ou de parler d'elle. On emploie généralement le nom de son aîné, fille ou garçon, parfois celui du cadet, si l'aîné est une fille et le second un garçon. Si un homme ignore le nom de l'aîné, il n'est pas inconvenant de lui substituer le nom de l'enfant qu'il connaît.

En comparant l'usage technonymique et l'usage patronymique, on comprendra aisément comment les formules de politesse révèlent le statut social des interlocuteurs. Les Nuer ont un mode de descendance patrilinéaire, et leur résidence est habituellement patrilocale. La femme acquiert son statut au domicile de son mari en lui donnant un enfant, aussi les gens du groupe social du mari s'adressent-ils normalement à une femme mariée par le nom de son fils aîné qui constitue le lien entre elle et eux. Avant la naissance de son premier enfant, il n'existe, au domicile du mari, aucune manière de saluer la femme, mais, comme elle vit avec ses parents jusqu'à son premier accouchement, il n'en résulte aucun inconvénient. Un homme est appelé en référence au nom de son fils aîné par la famille de sa femme, car l'enfant qu'il a engendré par leur fille représente le lien qui les unit, mais dans sa propre famille cette salutation s'emploie rarement. D'autre part, un homme est attaché au domicile et à la lignée paternels par son père, il est donc normal qu'on s'adresse à lui par un patronyme ; les femmes appartiennent à la maison de leur mari et à sa lignée, il est donc compréhensible que l'emploi des patronymes soit d'un usage peu courant en ce qui les concerne. De préférence, on parle d'un adulte mâle comme fils d'un tel et d'une femme adulte comme mère d'un tel. Mais chez ses grands-parents maternels, l'individu est lié aux gens par sa mère, d'où l'usage habituel d'un matronyme quand on s'adresse à lui. Les liens entre un homme et sa famille maternelle sont encore accentués par l'emploi d'un nom de naissance spécial et du nom honorifique du clan. Un garçon qui vit dans le village d'un homme qui le traite comme son fils, sans être luimême son pater, est appelé, dans ce village, du nom de cet homme, car c'est par lui qu'il est socialement lié à cette communauté, alors que dans le village de son pater, il porte le nom de ce dernier. Les différences de statut attachées à l'âge s'expriment aussi dans les titres de politesse, notamment dans l'usage des «noms de bœuf» par les compagnons du même groupe d'âge, et leur évitement entre gens d'âge différent. Ces titres de politesse fournissent aussi, comme nous l'avons vu, des indications quant au sexe, quant à certaines relations propres à la naissance des jumeaux, et quant à des situations sociales particulières. 


\section{Chapitre 11 \\ "Tribus et clans Luo" (1949)}

$\underline{\text { Retour à la table des matières }}$

Grâce à la fondation Leverhulme, j'ai eu en 1936 l'occasion de passer quelques semaines au Kenya. Étant donné les conditions particulières de cette époque, le gouvernement du Kenya jugea peu opportun que je me rende chez les Masai ou chez les Turkana, comme j'en avais l'intention. Aussi, décidai-je de m'occuper au mieux en rédigeant une étude sommaire sur les Luo, d'autant que les écrits consacrés à ce peuple étaient peu nombreux et souvent de qualité médiocre ${ }^{1}$.

Article paru dans le Rhodes-Livingstone Journal, 1949.

1 C. W. HOBLEY, Kavirondo, The Geographical Journal, 1898; Sir Harry JOHNSTON, The Uganda Protectorate, vol. Il, chap. XVIII, 1902; C. W. HOBLEY, Eastern Uganda, Occasional Papers of the Royal Anthropological Institute, $n^{\circ}$ 1, 1902; G. A. S. NORTHCOTE, The Nilotic Kavirondo, J.R.A.I., 1907; le Rév. N. STAM, The Religious Conceptions of the Kavirondo, Anthropos, 1910 ; C. M. DOBBS, Fishing in Kavirondo Gulf, J.E.A.U.N.H.S., 1927; le Rév. H. HARTMANN, Some Customs of the Luwo (or Nilotic Kavirondo) living in South Kavirondo, Anthropos, 1928; K. C. SHAW, Luo Marriage Customs, J.E.A.U.N.H.S., 1932; archidiacre W. E. OWEN, Food Production and kindred Matters amongst the Luo, J.E.A.U.N.H.S., 1933. 
J'ai passé quelque six semaines en pays luo. En raison du peu de temps dont j'ai disposé, abrégé en outre par une mauvaise fièvre, j'ai limité mes recherches à certains aspects de l'organisation sociale luo. Je me suis orienté plus spécialement vers une étude de la structure politique des Luo, afin de découvrir des similitudes possibles avec la structure politique des Lango, des Nuer, des Anuak et des autres peuples nilotiques. On connaît maintenant certains résultats de cette recherche. Ils auraient été publiés plus tôt si la guerre ne m'avait empêché de me consacrer à mes recherches anthropologiques. Les informations fournies dans cet article datent de 1936.

J'espérais que l'archidiacre W. E. Owen, qui connaissait les Luo mieux que quiconque, lirait cet article, mais il est mort en 1945. Je lui suis très reconnaissant ainsi qu'à Mme Owen, car, sans leur aide, je n'aurais pu mener à bien mon étude aussi rapidement. Je dois aussi remercier bien des Luo qui m'aidèrent dans mes recherches.

Le peuple nilotique des Luo du Kenya compte environ 500000 personnes, implantées en bordure du lac Victoria Nyanza, au nord et au sud du golfe Kavirondo. Quelques groupes isolés vivent au Tanganyika. Les zones du pays luo situées loin du lac, par exemple les régions Alego et Ugenya, sont vallonnées. Les maisons y sont construites sur les pentes (thuce, sing. thur qui signifie aussi «maison») des collines. Le pays qui borde le lac est moins accidenté. Partout les chutes de pluie sont importantes et bien réparties. La population, à dominante agricole, s'occupe aussi de l'élevage du bétail, des moutons et des chèvres, car la majeure partie du pays convient à la vie pastorale. La pêche joue un grand rôle dans l'économie des tribus habitant en bordure du lac. La chasse est peu pratiquée aujourd'hui, mais autrefois, on organisait régulièrement des battues.

La concession, résidence d'une famille restreinte ou étendue, est généralement désignée par le mot bantu dala ; mais, dans les régions peuplées plus particulièrement de Luo, le terme nilotique pacho (pl. mier) est utilisé, notamment par les anciens. Elle est habituellement entourée d'une haie épaisse d'euphorbes ; une ouverture étroite mène au kraal autour duquel se dressent les cases et les greniers. Les problèmes de la vie domestique et de la vie familiale dans la concession ne sont pas traités dans cet article visant seulement à décrire la nature des groupes structurellement plus importants.

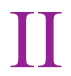

Retour à la table des matières

Le pays luo est actuellement divisé en un certain nombre de régions administratives, délimitées dans la carte (ci-contre). Leur surface et leur population varient considérablement. D'après les rapports officiels, la plupart comptent moins de 20000 habitants, deux seulement dépassent 30000 : la région Alego (53 604, densité 100 habitants au kilomètre carré) et la région Samia (41011, densité 77). La population atteint sa plus forte densité dans certaines régions fertiles du nord, dans le nord Ugenya (29 363, densité 206), le sud Ugenya (7 497 , densité 136), le nord Gem (29 014, densité 155). Les densités des plus faibles se rencontrent 
dans quelques régions situées au bord du lac, au sud du Kavirondo, dans le Kaksingiri (1 904, densité 5), le Kasigunga (2 041, densité 8) et le Gwasi (4 934, densité 17).

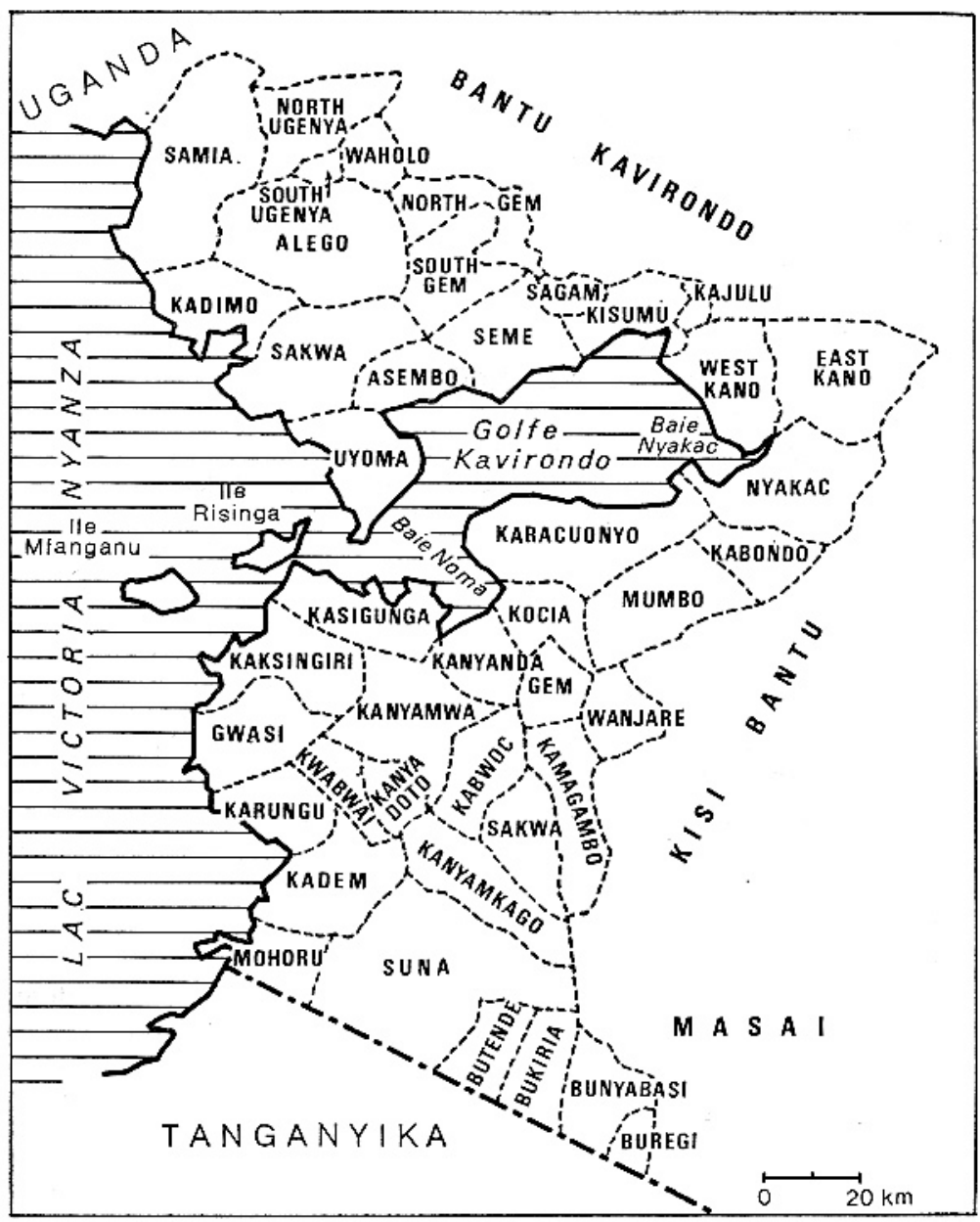

Distribution géographique et administrative des tribus luo et assimilées

Distribution géographique et administrative des tribus Luo et assimilées

Ces régions correspondent à peu près aux zones habitées par les anciennes tribus luo, à l'exception, au sud du Kavirondo, des régions Karacuonyo (28207, densité 67), Mumbo (26465, densité 70) et Kabondo (7 926, densité 50) occupées par une seule tribu, ainsi que l'étaient, au nord, les régions nord Gem et sud Gem (19 442, densité 95), nord Ugenya et sud Ugenya, ouest Kano (28 617, densité 106) et est Kano (25 730, densité 63).

Il est hors de doute que le pays luo fut autrefois entièrement habité par des tribus bantu et des tribus nilo-hamitiques. La pression continue exercée, presque jusqu'à nos jours, 
notamment contre les Bantu, aux confins nord, sud et est du pays luo, résultent d'un phénomène progressif d'expansion et d'assimilation, pendant lequel les Luo subirent très fortement l'influence de la culture bantu.

La progression des Luo vers le sud eut pour conséquence leur division en deux groupes reliés par l'étroite région Kisumu : les Luo du centre Kavirondo, appelés Joiye ou "gens de l'intérieur», et les Luo du sud Kavirondo, appelés Jooko ou "gens de l'extérieur». La migration vers le sud semble s'être effectuée en traversant le golfe, et non pas en le contournant, depuis les régions Uyoma et Asembo, en direction du Karacuonyo. Elle aurait débuté il y a environ sept générations, si l'on en croit les généalogies. De l'autre côté du golfe, les Luo se heurtèrent aux tribus bantu (Mwa) et nilo-hamitiques (Langu). La conquête et l'assimilation culturelle de ces tribus n'étaient pas encore achevées au moment de l'occupation européenne qui en ralentit le processus. Les tribus qui, aujourd'hui, au sud du golfe, seraient d'origine luo et en parlent la langue, sont les Kano, les Nyakac, les Karacuonyo (Mumbo, Kabondo et Karacuonyo), les Kocia, les Kanyada, les Gem, les Kanyamwa, les Karungu, les Kadem, les Kwabwai, les Kanyadoto, les Kabwoc et les Sakwa. Les autres régions indiquées sur la carte sont occupées par des tribus bantu dont certaines ne parlent que le luo, comme les Kagan (établis dans la région Kocia) et les Mohoru, tandis que d'autres connaissent à la fois le luo et leur propre langue, comme les Kasigunga, les Kaksingiri et les Gwasi. Par contre, la tribu des Wanjare, parente des Karacuonyo, est une tribu luo qui a adopté, dit-on, un dialecte bantu en remplacement de sa propre langue, car, selon le mythe luo, l'ancêtre de cette tribu tua son père dans un accident de chasse. Aussi, en raison de leur origine, les Luo considèrent les Wanjare avec plus de respect que les autres tribus de langue bantu. Les Kamagambo et les Kanyamkago seraient des tribus nilohamitiques bien que leurs membres s'expriment aujourd'hui en luo. Les tribus qui ne parlent désormais que le luo et qui ont adopté les coutumes de l'ethnie sont considérées comme Luo ; il serait injurieux d'appeler un de leurs membres Jamwa ou Langu. Les mariages entre Luo d'origine et Bantu partiellement ou totalement assimilés sont fréquents. Actuellement, les Luo épousent aussi des Bantu Kisii, mais, m'a-t-on dit, un Luo n'accepterait pas comme première femme une jeune fille de cette tribu. Dans le centre Kavirondo, l'assimilation dans les zones de conquête et de colonisation a été beaucoup plus profonde, et, mis à part le cas des Waholo qui parlent le luo et le bantu, la délimitation du clivage linguistique est beaucoup plus rigoureuse.

L'expansion luo aux dépens des Bantu pourrait être comparée à une manœuvre de wagons : chaque tribu poussait devant elle une tribu qui en poussait elle-même une autre, cette dernière habituellement bantu. Ainsi, quand les tribus Uyoma et Asembo occupèrent ensemble ce qui est maintenant le territoire uyoma, elles y trouvèrent les tribus Karacuonyo et Gem (du sud Kavirondo) qu'elles refoulèrent au-delà du golfe, dans ce qui était alors le pays bantu. Plus tard, les Uyoma chassèrent les Asembo dans leur territoire actuel. Ces mouvements provoquèrent une forte dispersion des lignages, de sorte que les lignages d'un même clan se rencontrent aussi bien dans le centre que dans le sud Kavirondo. On retrouve des lignages du même clan dans la tribu Gem du sud Kavirondo et dans la tribu du même nom du centre Kavirondo, des lignages de la tribu Kanyada possèdent une parenté agnatique avec le lignage Jokakan de la tribu Alego, et les éléments luo des tribus Kowak et Kamageta du Tanganyika proviennent, dit-on, des régions Kajulu et Kadimo où ils ont des parents. 
Rien de surprenant, par conséquent, à ce que les derniers venus qui refoulèrent les autres tribus luo en contact avec les Bantu avant de les conquérir, aient été les moins influencés par la culture, la langue et le mode de vie des Bantu. Les occupants des régions Uyoma, Seme et Asembo appartiennent à ce groupe. La plaine habitée par ces tribus est une savane épineuse, entrecoupée de zones dénudées, marécageuses et herbeuses, de laquelle le mot Seme tire son origine ; cette région souffre de sécheresse saisonnière, et rappelle le pays de certains Nilotes du Soudan, parents des Luo.

\section{III}

$\underline{\text { Retour à la table des matières }}$

Chaque tribu luo (piny) qui occupait, dans l'ensemble, comme je l'ai déjà noté, le territoire correspondant à une région administrative actuelle, constituait une unité politique auto

\section{LA FEMME DANS LES SOCIÉTÉS PRIMITIVES}

nome. L'une des principales caractéristiques de la tribu luo, comme d'ailleurs de la tribu nuer, et qui peut servir à définir son autonomie, apparaissait dans la manière de considérer et de traiter l'homicide avant l'arrivée des Européens. Il existait des particularités locales, mais, en général, le principe était le suivant: on ne payait une compensation, cul (une jeune fille ou un animal qui, offert à un parent du mort, lui servait à acquérir une femme dont les enfants héritaient du nom du défunt), que pour le meurtre d'un membre de la tribu. Et l'on ne versait rien pour le meurtre de membres des autres tribus, que ce soit lors des guerres, des raids ou des démonstrations rituelles, buru ${ }^{1}$, car ils étaient des ennemis, wasigu, et une hostilité permanente régnait entre les lignages, comme parfois un état de guerre entre les tribus. De même, aucune compensation ne pouvait être versée pour le meurtre d'un membre du clan, mais un parent du meurtrier fournissait un bœuf que mangeaient les représentants du clan tout entier, qui pouvaient contraindre le coupable à épouser une jeune fille au nom du défunt. Le sacrifice de ce bœuf servait d'amende honorable. Ainsi, l'obligation ou la non-obligation de payer une compensation ou d'offrir un sacrifice de réparation, en cas d'homicide, permettait, aux yeux des Luo eux-mêmes, de délimiter les clivages politiques entre tribus. Par exemple, tant que les peuples Uyoma et Asembo vécurent sur le même territoire, ils se payèrent mutuellement une compensation en cas de meurtre. Cette obligation disparut lorsqu'ils se séparèrent.

Si quelqu'un tuait un individu d'une autre tribu au cours d'une querelle personnelle, en dehors d'une guerre, d'un raid, d'une discorde ou d'une démonstration rituelle, il pouvait payer une compensation en bétail par exemple, lorsqu'un Karacuonyo tuait pour une raison particulière un Kocia qui venait amener du bétail à sa belle-famille habitant le territoire Karacuonyo. 
Dans les tribus dont tous les membres, à l'exception de quelques petits lignages d'étrangers (jodak) mêlés au clan dominant, appartenaient au même clan, comme dans la tribu des Karacuonyo, on ne versait que très rarement de compensation pour les homicides. Les étrangers y étaient étroitement liés au clan dominant, et, m'a-t-on dit, en compensation, ils auraient accepté une jeune fille mais refusé du bétail. Ce faisant, ils auraient cependant accentué la distinction qu'ils étaient désireux d'effacer. Le paiement du cul pour homicide n'était donc régulièrement effectué que dans les tribus qui comptaient, outre le clan dominant, d'autres puissants lignages (habitant le domaine tribal), comme les Jokakan et les Kaluo en pays alego. Le paiement pouvait alors être exigé par recours aux armes. Autrement dit, là où régnait une grande cohésion politique, exprimée en termes d'appartenance à un clan, l'homicide était considéré comme une faute; lorsque s'opposaient des intérêts particuliers à l'intérieur de l'unité politique de la tribu, on le traitait comme un préjudice ; enfin, dans le cas de liens sociaux combinés avec une indépendance politique, à moins qu'il ne s'agisse d'un acte individuel et gratuit, on le jugeait comme une provocation d'hostilité, mais sans désapprobation morale ni moyen de règlement légal.

La frontière politique, qui moralement était synonyme d'hostilité équilibrée entre deux groupes tribaux, s'exprimait matériellement dans le thim. Le thim était une portion de brousse entre une tribu et ses voisines, une sorte de no man's land qui devenait le domaine du buffle, du lion, du léopard et de l'éléphant. On rencontrait ces thim entre les territoires de deux tribus, par exemple entre la région Alego et la région Gem, mais non pas entre les divers segments d'une tribu, même lorsqu'ils appartenaient à des clans différents, par exemple, il n'y avait pas de thim entre les Joseje, les Jokakan et les Jokaruoth de la tribu Alego. Le thim, parfois très vaste, pouvait atteindre 15 kilomètres de large.

Les tribus opéraient des raids les unes contre les autres à travers le thim qui les séparait, c'est là qu'avaient lieu les démonstrations rituelles du tero buru. Quand mourait un haut dignitaire de la tribu, un notable ou un guerrier renommé, ou lorsqu'un homme était tué à la guerre, on ne l'enterrait pas avant que toute la tribu, ou au moins une portion importante de celle-ci, n'ait traversé le thim, fait une démonstration devant les concessions d'une tribu voisine, exhibé son bétail, injurié et insulté les hommes afin qu'ils sortent se battre. La présence du bétail avait pour but d'amener l'ennemi à combattre en lui offrant un butin à portée de la main, mais aussi de forcer le groupe buru a ne pas perdre de terrain si on l'attaquait car il était indigne d'un pasteur de ne pas défendre son troupeau jusqu'à la mort. Ainsi, les Uyoma avaient l'habitude de marcher contre les Sakwa; les Seme contre les Asembo, les Kisumu et les Gem; les Gem du sud du golfe contre les Kamagambo, les Kabwoc, les Karacuonyo et les Kanyamwa ; les Karacuonyo contre les Kocia, les Gem, les Nyakac, les Bantu Kisii et même les Lumbwa. Lorsqu'une tribu luo jouxtait une tribu bantu, elle pratiquait, contre cette dernière, le buru, de la même façon que les tribus luo entre elles ; les tribus bantu du Kavirondo possédaient une structure du même ordre que celle des tribus luo. La tribu qui se manifestait n'était pas habituellement attaquée, sauf lorsque deux groupes buru de tribus opposées se rencontraient et déclaraient leur intention de défi réciproque, ou lorsque la démonstration se transformait en un raid contre le bétail, ou lorsque les concessions étaient mises à sac. Cette coutume a été conservée, mais elle se réduit à une escarmouche factice; on conduit le bétail dans le thim où l'on écrase des brindilles et des herbes. On rapporte des poignées de plantes qui représentent le bétail capturé et on les jette sur le toit de la case du défunt. 
Il n'existait aucune fonction que l'on pût qualifier de politique dans une tribu luo. Le ruoth n'était qu'un homme influent, sans plus. Il possédait plus de femmes et de bétail que ses compagnons et les gens venaient souvent chez lui pour y manger et pour y discuter les affaires du jour. Un pauvre, m'a-t-on dit, pouvait solliciter de lui une vache qui l'aiderait à acquérir une femme, et on considérait comme un honneur d'épouser quelqu'un de sa famille. Tout homme qui atteignait une position sociale supérieure était appelé ruoth, mais il appartenait, la plupart du temps, au clan dominant de la tribu. Certains guerriers (thuondi, taureaux) acquéraient une renommée de courage et d'efficacité à la guerre, et on leur demandait de conduire des raids en territoire ennemi. Même s'il était admiré, un chef guerrier, appelé parfois thuon jatela, n'était pas nécessairement un ruoth, c'est-à-dire un notable aisé, car il ne recevait qu'une faible part du butin de la guerre, une seule vache par exemple. Cette position n'était pas héréditaire, elle découlait des exploits et des initiatives personnels. Le won gweng, «père de la terre », semble avoir été, au moins en certaines régions du pays luo, un homme influent du district. C'était un ancien du lignage du clan dominant de la tribu, qui réglait les litiges fonciers. Avant une guerre tribale, le représentant de la branche aînée du clan offrait un sacrifice à la lance sacrée de ce clan et la pointait en direction de l'ennemi.

Parmi les nombreux spécialistes des rites, le plus important, en même temps la figure la plus influente de la tribu, semble avoir été, chez les Luo, le jabilo, équivalent du guérisseur (medecine-man) de la littérature africaine. Il en existait un dans chaque segment de tribu, mais, dans chaque tribu, l'un d'eux possédait une influence supérieure aux autres. Si l'on avait besoin des services d'un jabilo exceptionnellement renommé, on pouvait faire appel à celui d'une tribu même très éloignée. Ses fonctions

qui tendaient à se perpétuer de père en fils consistaient à prévoir l'issue de la guerre, à jeter sa malédiction sur l'ennemi, à fabriquer des talismans pour assurer la victoire et la sécurité des guerriers. Il gardait son talisman de guerre, bilo, à l'intérieur de cornes remisées dans un grenier. Il ne s'associait pas aux raids, mais il recevait, en échange de ses services rituels, une partie du butin, et, selon certaines informateurs, on lui confiait le soin du partage. En conséquence, c'était un ruoth, un homme riche. Si quelquefois le jabilo assumait les fonctions de faiseur de pluie, jokoth ou won koth, parfois une autre personne en remplissait l'office. On réclamait aussi les services des faiseurs de pluie des peuples bantu voisins. Les gens devaient, dit-on, attendre que le jabilo donnât la permission de semer. Il avait des fonctions rituelles en cas de meurtre à l'intérieur de la tribu. Lors des combats, il jouait un rôle de médiateur, en plaçant à même le sol, entre les deux partis opposés, sa peau de léopard. 


\section{IV}

$\underline{\text { Retour à la table des matières }}$

Dans chaque tribu, domine un clan ou un lignage. Les caractéristiques du clan luo sont sensiblement les mêmes que celles du clan nuer.

Le clan luo (dho ot) possède une structure lignagère, c'est-à-dire que chaque membre d'un clan peut retrouver sa relation généalogique exacte avec n'importe quel autre membre de son clan. Les lignages (dho udi) se différencient par l'adjonction, au nom d'un clan ou d'un lignage plus important, du copulatif ma, suivi du nom d'une branche secondaire, ainsi, chez les Lango, les Joseje ma Jokayila constituent la lignée principale Jokayila du lignage-souche Joseje à l'intérieur du clan JoAlego. Les lignages collatéraux sont parfois appelés kanyiego, terme qui tire probablement son origine de nyieka, c'est-à-dire coépouse.

Les clans les plus importants associés aux territoires tribaux ne sont pas toujours, à l'heure actuelle, exogames, mais on pense qu'ils l'étaient autrefois. Il serait déroutant de désigner deux groupes de structure identique par des termes différents, sous prétexte que, dans l'un, les lignages principaux ne peuvent contracter de mariages entre eux, tandis qu'ils le peuvent dans l'autre, aussi j'utiliserai le mot «clan » pour les deux groupes, après avoir signalé la modification apportée à l'usage habituel du terme. Le mariage est généralement interdit entre membres d'un même clan, cependant, la distance entre les lignages principaux peut devenir telle, tant du point de vue généalogique que politique et social, que les mariages soient autorisés. C'est ce qui se produisit dans le clan JoAlego des Alego, dont les membres de deux lignages principaux, JoSeje et KaDenge, se marient entre eux après s'être querellés. D'autre part, le mariage n'est pas autorisé à l'intérieur de clans importants, comme ceux des KaNyikal du Seme, des KaRacuonyo du Karacuonyo, des JoOwila de l'Uyoma; on ne l'approuve pas non plus, malgré quelques exceptions, entre les clans frères, par exemple, entre les clans dominants du Karacuonyo, du Kisumu, du Kadem, du Karungu et du Kabwoc. Les jeunes se révoltent contre cette interdiction. De même, bien que les JokOwila contractent des mariages dans le clan des Omia (Asembo), on craint le peu de fécondité de telles unions.

Cependant, à l'intérieur de ces vastes clans où le mariage est interdit, ainsi qu'entre les clans frères qui le désapprouvent, la coutume du codo est autorisée. Cette coutume sociale du codo permet aux jeunes gens de courtiser les jeunes filles, de les emmener dans la case des célibataires, simba. Là, ils couchent ensemble et ont des rapports sexuels, intra crura. La pratique du codo est acceptée entre les lignages KaAbudha et KoOkelo du clan JoOwila de l'Uyoma depuis deux générations, tandis qu'entre les lignages KaGumba et KaDepir du clan KaNyikal du Seme, elle remonte à la dernière génération seulement, ainsi qu'entre les JokOwidi et les Jok0gol du clan KaRacuonyo du Karacuonyo. Si une jeune fille se trouvait alors enceinte, elle ne pourrait épouser son amant. 
Quand deux lignages d'un même clan deviennent très éloignés l'un de l'autre, et qu'ils commencent à pratiquer le codo, ils le font d'abord avec discrétion, car les anciens s'opposent à cette innovation, puis ouvertement dans la case des célibataires. Pour finir, le mariage est envisagé, puis autorisé, d'abord sous sa forme non sanctionnée, par enlèvement (por), puis sous sa forme habituelle (meko). Lorsque les unions jusqu'alors interdites commencent à se produire, à plus forte raison quand elles reçoivent l'approbation des anciens, ce peut être le signe d'une fission politique, car on a remarqué que, chez les Luo comme chez les Nuer, les valeurs politiques sont évaluées en termes de parenté et que le système politico-territorial se reflète dans le système lignager.

Un homme ne peut épouser une fille de sa sœur, nyakewo, au sens classificatoire, si la mère de cette jeune fille est membre du lignage principal auquel lui-même appartient, mais il peut l'épouser si la mère fait partie d'une branche collatérale du lignage principal de son clan, et même si les anciens n'approuvent pas le mariage. Le principe général d'exogamie est le suivant: un homme ne doit pas épouser une femme qu'il considère comme une parente cognatique, wat, et, pour les Luo, ce principe de parenté empêche un individu d'épouser les femmes du clan de sa grand-mère maternelle et celles du lignage principal de son arrièregrand-mère maternelle. Les désaccords abondent, surtout entre hommes de générations différentes, à propos de l'autorisation de se marier avec des parentes éloignées, en particulier dans les cas limites. En dépit de quelques divergences d'opinion, on peut dire que, pour les Luo, les gens sont ou bien des parents, wat, ou bien des ennemis, wasigu. Un homme ne peut épouser ses parentes, il doit donc épouser une «ennemie ». Dès lors, si le mariage est autorisé entre deux groupes, c'est que n'existe entre eux aucune parenté, ou bien que cette parenté n'est plus reconnue du point de vue de l'exogamie. La compatibilité matrimoniale constitue un signe d'opposition politique, comme je l'ai déjà mentionné, si bien que l'on entend souvent les Luo dire, en parlant d'une tribu voisine, par exemple un Karacuonyo parlant des Kocia : «Ce sont nos ennemis, nous les épousons. » En d'autres termes, des tribus voisines sont des segments structurellement opposés dans le système tribal, mais, à travers leurs frontières communes, des relations interpersonnelles et des liens de parenté entre individus se nouent, qui sont nécessaires étant donné les règles de l'exogamie en vigueur dans la société luo.

Plusieurs clans et lignages principaux possèdent des lances et autres objets sacrés qui ont appartenu, croit-on, à leurs fondateurs. La lance sacrée la plus célèbre (long ma lisua, lance du sacrifice) est la lance d'Alego, le fondateur du clan JoAlego. En pays ugenya, les JokaGer détiennent plusieurs lances similaires, de même que, au sud du golfe, les lignages descendant de Cuanya. Les principaux lignages étrangers d'une tribu peuvent aussi avoir leurs objets sacrés, en pays alego, les KaLuo et les JokOgelo conservent des lances sacrées ; quant aux JoAgoro, ils possèdent une pierre et une marmite sacrées transmises par leurs fondateurs. Ces objets sont les symboles de l'unité d'un clan ou d'un lignage, et, par extension, de la tribu ou du segment tribal dans lequel ce clan ou ce lignage prédomine. Ils étaient utilisés dans des rites en temps de guerre ou de désastre collectif comme la sécheresse ou l'invasion des criquets. Il semble qu'ils aient généralement été détenus par la branche aînée (dho ot ma duong) d'un lignage ou d'un clan, et qu'ils aient été, soit conservés dans un grenier, soit cachés dans la brousse. La branche aînée est souvent sollicitée par les branches cadettes collatérales pour accomplir des sacrifices en leur nom. C'est elle qui doit fournir les animaux du sacrifice lors des cérémonies communes. Tandis que tous les lignages ont leur «père de la terre », won gweng, le won gweng de la branche aînée jouit d'un prestige supérieur. 
Certains clans et lignages ont des interdits rituels (kwer), surtout alimentaires, touchant notamment la consommation de rognons et de foie. Les Luo ne semblent pas y attacher une grande importance, et une identité de tabou pour un homme et une femme ne constitue pas un empêchement au mariage. Je dois aussi faire allusion aux noms honorifiques des clans et des lignages. Le nying paak provient généralement du nom d'un ancêtre paternel qui sert de nom de famille au lignage, par exemple Okwiri (cf. le tableau généalogique du clan JoOwila) peut être appelé, selon les circonstances, wud (fils de) KoOkelo, wud KaJeje, wud Owila. On emploie beaucoup ces noms lors des réunions communes, spécialement aux fêtes de la bière.

Un trait du système clanique, qui semble revêtir une certaine importance, est la lutte annuelle entre lignages. Malheureusement, je ne pris connaissance de cette coutume que peu avant mon départ du pays luo, et n'eus par la suite aucune occasion de compléter mon information, ni de déterminer si sa pratique était générale ou propre à quelques régions tribales seulement, telles que l'Uyoma, où les lignages collatéraux proches parents luttent pour s'entraîner avant les combats entre grands lignages. Ainsi, les KoOkwiri luttent contre les KoObong, puis ces deux lignages s'unissent et se battent contre les KaAtuenga et les KaAbudha, etc. Les lignages envoient un messager pour défier l'autre parti. Lors de la rencontre, un ancien arbitre les assauts et les exhibitions entre les plus célèbres lutteurs des deux partis. Les matches provoquent une intense excitation parmi les spectateurs.

Cette description du clan luo ne constitue qu'une esquisse dégageant quelques caractéristiques. La section suivante ajoute des détails sur le clan JoAlego de la tribu Alego, le clan JoOwila de la tribu Uyoma, le clan KaNyikal de la tribu Seme et le clan KaRacuonyo de la tribu Karacuonyo. Il convient de conserver en mémoire, à propos de ces exemples et des règles de l'exogamie mentionnées ci-dessus, que ces clans sont associés avec des territoires tribaux, qu'il existe beaucoup de petits lignages, la plupart d'origine bantu, à l'extension territoriale plus réduite et de moindre profondeur lignagère; et aussi que les principaux clans descendent tous, en définitive, des mêmes ancêtres mythologiques Podho et Ramogi et qu'ils pourraient donc tous figurer sur un même tableau généalogique. Comme les lignages étrangers de moindre importance ont aussi des points d'implantation sur les arbres des clans dominants, le peuple luo tout entier pourrait être représenté sur un seul tableau. Les clans que j'ai décrits forment cependant les clans généalogiques les plus importants rattachés à des ancêtres éponymes que les Luo considèrent comme les limites à partir desquelles les données généalogiques exactes disparaissent dans les brumes de la tradition, où une seule lignée d'ancêtres mythologiques se perd finalement dans les origines du monde. Ils constituent les points de départ de la structure généalogique. 


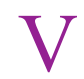

$\underline{\text { Retour à la table des matières }}$

Dans la tribu Alego, dominent les clans JoAlego dont les lignages principaux sont les JoSega et les KaDenge. Il existe, sur le territoire tribal, hormis quelques petits groupes d'étrangers, un certain nombre d'autres clans et quelques vastes lignages. Les deux clans étrangers les plus puissants sont les Karuoth et les Kakan (JokAnyango). Il faut citer, parmi les autres clans ou lignages principaux : les KaLuo, les JoMar, les JoAgoro, les JokOgelo, les KaNyinek, les KaRapol (JoKato), les JoUmala, les KaLikada, et les JoUyawa. La présence de ces clans et lignages en pays alego, leurs relations intertribales avec les JoAlego, se manifestent à travers nombre de traditions et de mythes. Ces mythes relatent qu'Alego, venant de l'ouest, avec son peuple, des pays Yimbo ou Kadimo, pénétra dans la région, y trouva un peuple nommé Kombekombe et qu'ils vécurent dans une entente mutuelle. Puis les Kombekombe commencèrent à se disperser et disparurent. Le pays tout entier revint alors à Alego et à ses fils. Lorsque d'autres clans voulurent le suivre dans ce pays, Alego les combattit et les contraignit à s'installer ailleurs. Les fils aînés de Mulijuok traversèrent ensuite le golfe et se fixèrent dans ce qui est maintenant le sud Kavirondo. Mulijuok resta avec ses plus jeunes fils, Seje et Nyidenge, et ses trois filles, Nyiwat, Anyango et Ajonga.

D'autres clans, autorisés par les Alego (selon leur propre version) pénétrèrent dans le pays, tout d'abord Ragak qui épousa Nyiwat et Anyango, et qui fonda le clan des Kakan, considéré comme matrimonialement allié (orche) des JoAlego. Puis vint Ager (de Kadhola ou de Budama) qui demanda à Mulijuok la permission de s'établir. Celui-ci l'autorisa à occuper, avec son peuple, la colline Akara. Ager, à la mort de Mulijuok, décida de supprimer ses fils, Seje et Nyidenge, pour s'emparer de leurs terres. Seje eut vent du complot et fit appel à Nyinek, Uyawa, Agoro, Ragak et d'autres (dans le récit, les lignages sont personnifiés), pour combattre Ager et son peuple qu'ils vainquirent. Ager demanda, plus tard, la main d'Ajonga dont les enfants sont à l'origine du clan des Karuoth considérés eux aussi comme parents par alliance des Alego. La généalogie ci-dessous illustre ce récit. De Mulijuok à nos jours, on compte neuf ou dix générations. D'autres histoires, que je m'abstiendrai de relater, expliquent les rapports entre les Kakan, les JoUyawa et les autres clans du territoire tribal alego. Dans ces mythes, la structure tribale apparaît en termes de lignages personnifiés.

La généalogie JoAlego a été présentée sous sa forme la plus simple, dépouillée de toutes les branches secondaires. La description plus complète du clan JoOwila illustre mieux la structure lignagère d'un clan Luo typique. Les JoOwila constituent le clan dominant en pays uyoma (pop. 11694, densité 36,5). L'ancêtre du clan, Owila, vint du pays alego en amenant avec lui les divers clans qui vivent maintenant en pays Uyoma, ainsi que les peuples qui occupent le pays asembo (dont le clan dominant est celui des Kale). Ces derniers furent repoussés en pays asembo, par les peuples de l'Uyoma, lors d'une querelle. Bien que les 
lignages étrangers soient nombreux en pays uyoma, la population y est probablement moins mélangée que dans la plupart des territoires tribaux.

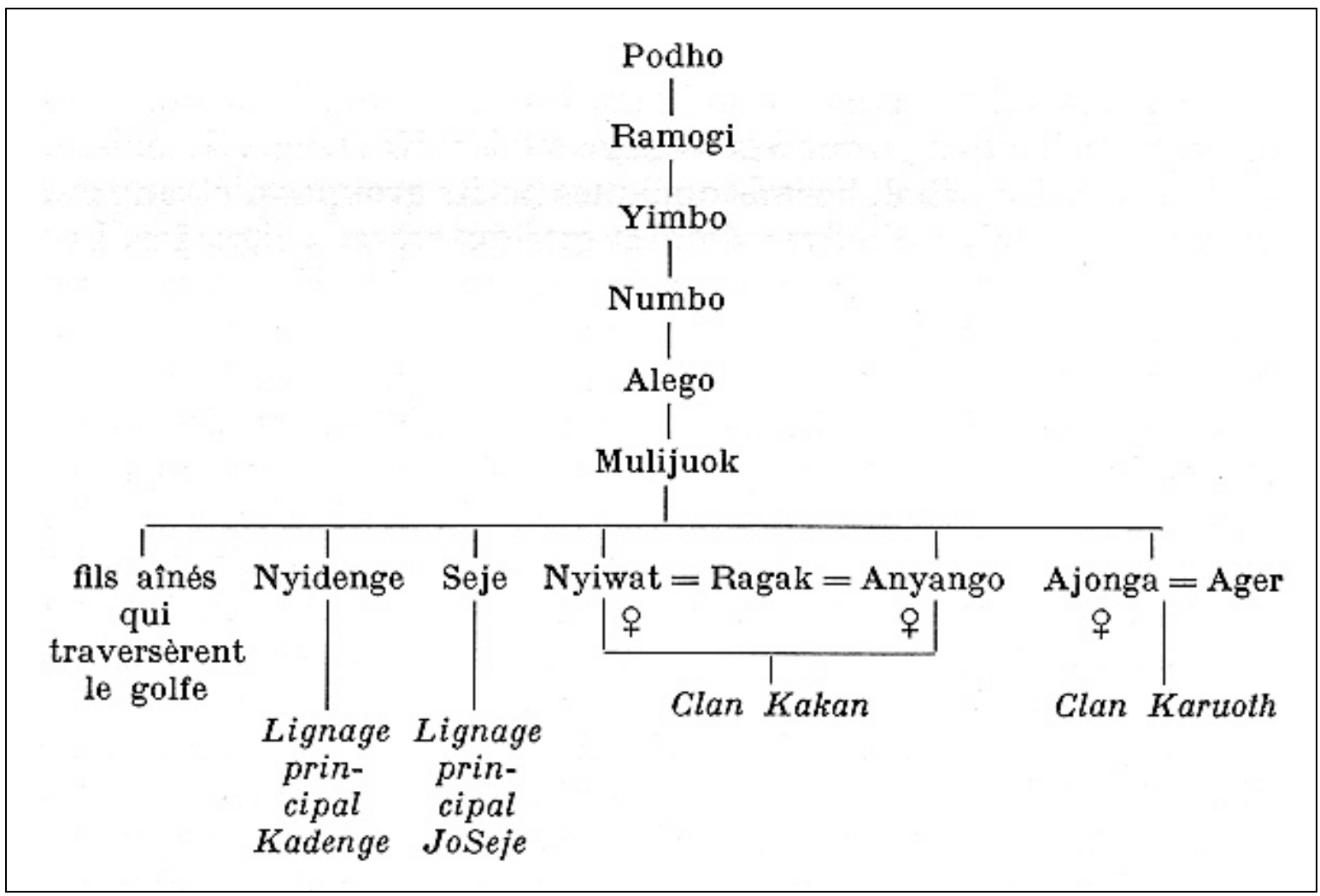

Le clan JoOwila se divise en quatre branches importantes ou lignages principaux, comme le montre le tableau ci-dessous, les JokOkelo ou JokObong constituent la branche aînée. Les membres de ces quatre lignages ne se marient pas entre eux. Chacun possède une portion du territoire tribal, bien que l'on rencontre souvent des membres d'un lignage vivant sur le domaine d'un autre. Les KaAtuenga et les KaAbudha qui ont une même aïeule se considèrent comme un seul segment, face aux KoOkelo et aux KoOkwiri. Le tableau ne fait pas état des liens généalogiques créés par des femmes de lignages étrangers vivant au sein du clan.

L'exemple du clan JoOwila peut servir à illustrer la relativité des valeurs lignagères à laquelle je me suis intéressé dans mon ouvrage sur les Nuer. Les noms de lignages sont en italique. Ce tableau ne présente qu'un seul lignage complet. Si on demande à Okwiri à quel lignage de son territoire tribal il appartient, il répondra probablement dho odwa KoOtual, « au lignage des KoOtual ». Si l'on poursuit : « A quelle branche des KoOtual ? (KoOtual ma dho ot mani), il répondra: KoOtual ma KaJeje, et s'il s'agit, dans le premier exemple, d'un cas où Kajeje s'oppose à JokOrua, il donnera sans doute le lignage Kajeje comme le sien. Si l'on se réfère aux JokAkech ou à un autre lignage médian, il se dira KoOtual, tandis que si l'on considère les KaAbudha ou un autre lignage principal, il se désignera comme KoOkelo. Cette caractéristique des systèmes lignagers est trop connue pour qu'on s'y attarde davantage. 


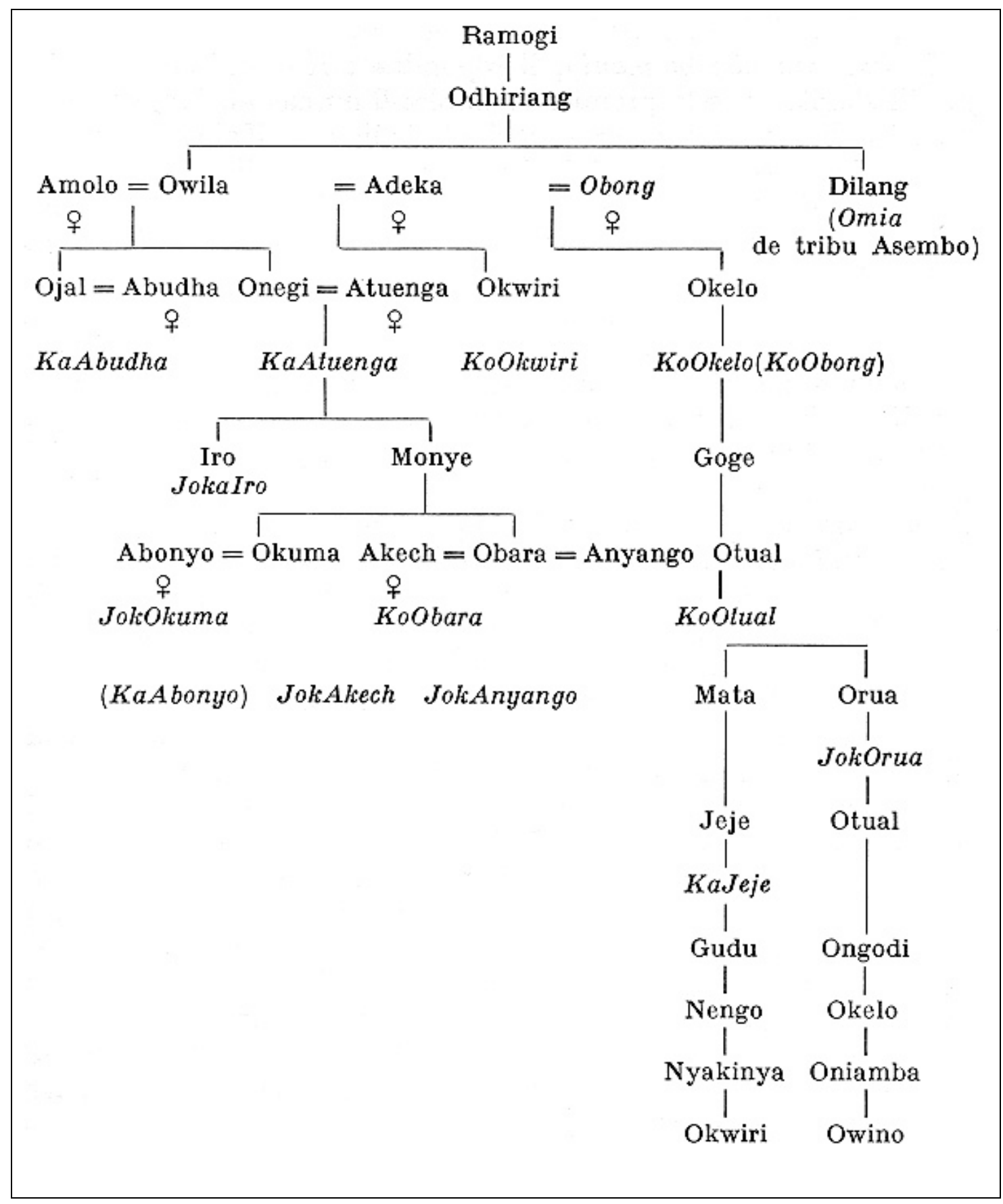

En pays Seme (pop. 29 919, densité 124,6), le clan dominant est celui des KaNyikal subdivisé en deux lignages principaux, les Kadepir et les KaGumba. Gumba était un enfant trouvé, adopté et élevé par Nyikal. Faute de place, je ne dresse pas ici la généalogie de ce clan, mais je voudrais, en passant, attirer l'attention sur une caractéristique commune des systèmes lignagers, à savoir, le processus de greffage des lignages étrangers, tel que le présentent les mythes, dans la structure du clan dominant de la tribu. Au lignage fort puissant 
des KaGumba, dont je viens de parler, s'en ajoute un autre important en pays seme, celui des KaYila. D'après la mythologie, Yila appartenait à la tribu des Alego. Il emmena, dans la hutte des célibataires, la fille de Nyikal, Amolo, qui se retrouva enceinte. Yila quitta alors le pays. Amazone de fort caractère, Amolo partit plus tard à la recherche de son amant qu'elle ramena en pays seme avec les fils d'une femme épousée durant son exil, et qui furent à l'origine de nouveaux lignages. Un autre clan du Seme est celui des Kolunje. Nyikal avait un serviteur nommé Anam qui conduisait régulièrement ses fils au nord du lac afin d'y ramasser du fourrage pour les veaux. Un jour, ils y trouvèrent un homme appelé Onyango. Nyikal adopta cet étranger avec Anam, et le nom de Kolunje désigne collectivement leurs descendants, les KaAnam et les KoOnyango.

Le dernier clan dont je vais donner un aperçu est celui des KaRacuonyo. Jok, qui semble s'identifier avec le Mulijuok du mythe alego, engendra deux fils. L'un d'eux, Cuanya, est l'ancêtre des principaux lignages des territoires tribaux du Kisumu, du Kanyamwa, du Kabwoc, du Karungu et du Kadem. L'autre est l'ancêtre du clan des KaRacuonyo qui domine dans les régions Karacuonyo (Kaduong), Mumbo et Kabondo. Selon le mythe alego, existaient, comme nous l'avons noté, deux autres fils, Seje et Nyidenge qui ne figurent pas dans les généalogies des peuples du sud du golfe. D'après certains, l'ancêtre des Wanjare, qui parlent maintenant bantu, était un autre fils de Jok.

Selon l'histoire orale du sud du golfe, Jok aurait été tué par ses fils lors d'une chasse à l'éléphant. Un homme nommé Cien prit avec lui Racuonyo en tant que domestique et, après diverses aventures communes, l'adopta comme son fils. A la mort de Cien, Racuonyo épousa l'une de ses veuves, Omiri, qui lui donna Sepol, ancêtre des lignages dominants dans les sections tribales du Kabondo et du Mumbo, les JokOwidi, et les JokOmala. D'après la tradition luo, Sepol était le fils du défunt Cien et non de Racuonyo, mais les fils de Cien refusèrent qu'il portât le nom de leur père, aussi fut-il appelé du nom de Racuonyo. Les descendants de Cien et d'une autre de ses femmes prétendent que les terres Mumbo leur appartiennent, mais ils ne constituent qu'un peuple de faible dimension, parce que, dit-on, ils insultèrent leur père qui revint les hanter après sa mort. Les descendants de Racuonyo apparaissent sur le tableau ci-après. Seuls figurent les principaux lignages à l'exception d'un seul cas servant d'illustration.

Selon le contexte, Rapemo peut se désigner comme appartenant aux lignages KaMuma, JokOnyango, KaNdaya, KoOkoth, JokOwidi, KaSepol et KaRacuonyo.

Chacun des grands lignages du clan KaRacuonyo possède sa propre portion de territoire tribal, et, à l'intérieur de la section occupée par un vaste lignage, les domaines des différentes branches se distinguent nettement. Si le mariage à l'intérieur du clan n'est pas autorisé, les mariages interclaniques provoquent la disparition relative des segmentations territoriales.

Il existe un certain nombre de lignages étrangers en pays karacuonyo (les Wagwe, les Kamwala, les Kakmuno, les Jokadero, etc.), mais de peu d'importance, et la fréquence des mariages avec le clan dominant efface toute distinction territoriale ou politique. On doit maintenant accorder quelque attention au statut des lignages étrangers face au lignage dominant à l'intérieur d'une tribu. 


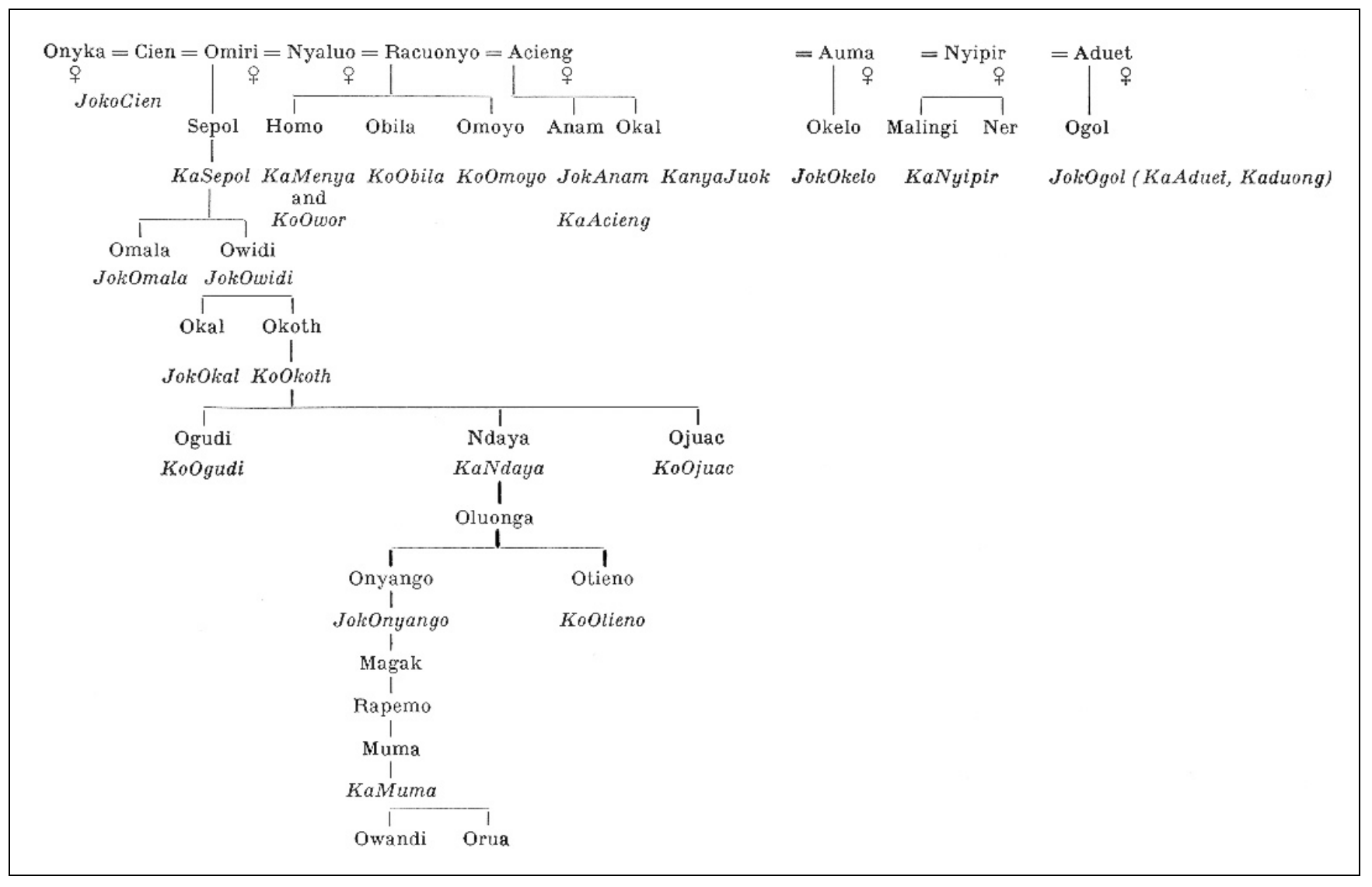

\section{VI}

$\underline{\text { Retour à la table des matières }}$

Nous avons noté le petit nombre et le peu d'envergure des lignages étrangers en territoire karacuonyo. Les membres du clan dominant constituent de beaucoup la grande majorité de la tribu. Certains territoires tribaux offrent un mélange clanique plus large, et la présence des lignages étrangers puissants et numériquement importants se reflète dans les mythes tribaux. Un Européen ne perçoit pas toujours immédiatement l'existence de ces éléments étrangers, à moins que quelque querelle territoriale n'attire sur eux l'attention, car le clan dominant est, en général, linguistiquement identifié à la tribu. Le terme JoAlego désigne à la fois les membres de la tribu occupant le territoire tribal alego et les descendants patrilinéaires d'Alego, de même que le mot KaRacuonyo désigne les membres de la tribu habitant le territoire tribal karacuonyo et les descendants patrilinéaires de Racuonyo. Donc, si l'on demande à un homme du lignage étranger JoMur du territoire alego, alors qu'il se trouve en dehors de ce 
territoire, à quel peuple il appartient, il répondra qu'il fait partie du peuple alego, mais si cette question lui est posée à l'intérieur du territoire alego, il dira qu'il est un JoMur, car les JoMur constituent un groupe agnatique distinct qui vit sur son propre domaine territorial en pays alego.

Sous un autre angle, seuls les membres du clan dominant dans un territoire tribal se considèrent comme les jopiny ou jogweng, c'est-à-dire les possesseurs du sol, les autres sont les jodak, les étrangers ; cependant, les membres du clan dominant appelleront les étrangers jebedo si ces derniers vivent depuis longtemps parmi eux, le mot jodak étant péjoratif.

Ces lignages étrangers se greffent sur la structure lignagère du clan dominant, de telle sorte que si l'on tient compte des liens créés par les femmes, la tribu tout entière peut être considérée comme un système lignager cognatique. Les Luo désignent les lignages affiliés par le terme kaorce, ou lignages par alliance, et par le mot kaner les lignages des oncles maternels, c'est-à-dire les lignages d'origine de leurs femmes et des mères de leurs enfants. Comme les lignages affiliés prennent leurs femmes dans le clan dominant, ils appartiennent à la catégorie des okewa, les fils de la soeur. Ainsi tout lignage d'une tribu, qu'il fasse partie du clan dominant ou qu'il soit d'origine étrangère, a une position structurelle dans le système des lignages qui composent la tribu et dont ceux du clan dominant constituent le cadre.

Il semble que, dans le passé, les lignages du clan dominant, possesseurs du territoire tribal, et les lignages affiliés aient vécu en égaux du point de vue social et aient agi en coopération dans les affaires tribales d'intérêt commun, particulièrement lors des guerres contre les autres tribus. Les différences n'intervenaient que dans les problèmes de rituel, de régulations des mariages, et, comme nous l'avons vu plus haut, dans la manière de traiter les cas d'homicides. Cependant, au cours des dernières années, dans les régions où la densité de la population est la plus forte, notamment parmi les tribus les plus au nord du centre Kavirondo, les différends graves ont surgi, à propos de la terre, entre les divers lignages, parfois entre un lignage du clan dominant et un lignage étranger, parfois entre deux lignages étrangers.

Dans le passé, aucune difficulté sérieuse ne semble s'être manifestée au sujet de la terre ; celle-ci abondait et il était Possible d'en acquérir par la conquête ou l'émigration. Les gens étaient heureux, dit-on, d'accueillir des étrangers qui augmentaient la force combattante de leurs communautés et pourvoyaient leurs fils en femmes. Les étrangers s'installaient sous le nom d'orce, parents par alliance, et devenaient des oncles maternels ou des fils de la sœur. Les relations personnelles entre les membres d'un lignage dominant et les étrangers qui s'établissaient parmi eux et avec lesquels ils contractaient mariage, étaient toutes des relations de parenté. Elles forment aujourd'hui les nœuds structurels où les lignages affiliés se greffent sur l'arbre généalogique du clan dominant. Les relations personnelles cèdent la place aux relations structurelles. Les gens ne se querellent pas facilement, pour de la terre, avec leurs propres parents par alliance, leurs oncles maternels, les fils de leurs sœurs, mais quand la pression de la population se fait trop vive, il se peut qu'ils se disputent avec les membres des lignages par alliance, des lignages des oncles maternels, des lignages des fils de leurs sœurs, c'est-à-dire lorsque la relation structurelle s'est substituée à la relation intime de parenté, car les mariages, et donc la formation de nouveaux liens de parenté, entre un lignage et un autre vivant sur son territoire tribal, doivent aller en régressant pour des raisons d'exogamie. L'appellation « fils de la sœur », employée par un membre du lignage JoSeje des Alego vis-à- 
vis d'un individu d'un lignage étranger, diffère énormément selon qu'elle indique un rapport personnel ou un rapport structurel. Dans les régions tribales surpeuplées, on observe aujourd'hui de nombreux litiges fonciers entre lignages. Bien qu'il ne s'agisse pas d'un trait nouveau dans la vie luo, il faut reconnaître que l'influence européenne l'a certainement accentué. La cohésion tribale a souffert de la paix, et l'appât de la terre a accru les dissensions.

Les jopiny réclament la terre en tant que propriétaires du territoire tribal, et montrent leurs anciens lieux d'habitation, gundini, dont les générations se souviennent longtemps, et les tombes de leurs ancêtres, dans le domaine revendiqué. Mais les lignages étrangers, dont le nombre et la puissance se sont accrus, peuvent se prétendre jopiny du même domaine, car ils y ont résidé pendant de nombreuses générations, et peuvent aussi y montrer leurs lieux d'habitation ancestraux et les tombes de leurs aïeux. Il arrive qu'un second lignage étranger ait pris de l'ampleur aux dépens d'un premier qu'il a chassé de ses terres, si bien qu'alors, trois lignages différents font valoir leurs droits sur un territoire. Lors de ma visite en pays alego, des problèmes de ce genre excitaient les passions, car la terre y devient rare. Ces problèmes peuvent encore se compliquer par le fait que l'actuel clan dominant a parfois, dans le passé, évincé du territoire le clan dominant d'alors, qui a donc, lui aussi, sur cette terre, ses lieux d'habitation et ses tombes. Chez les Luo, on considère que les anciens lieux d'habitation et les tombes ancestrales constituent les titres de propriété, et qu'une personne a le droit de demander une terre à cultiver dans le pays où vécurent et où sont enterrés ses ancêtres. Cette règle n'implique pas qu'un homme puisse quitter le domaine tribal sur lequel vit sa famille depuis plusieurs générations pour revenir sur celui qu'occupèrent ses ancêtres lointains, et réclamer la terre sur laquelle l'habitation, gunda, de l'un d'eux était située ; mais elle signifie qu'il a le droit de cultiver la terre quelque part dans ce pays. Il y a donc chevauchement des droits dans un même territoire.

Parmi les Luo, la propriété de la terre cultivable est assignée à un lignage, et chaque membre du lignage qui possède un domaine a le droit d'y travailler. L'individu n'a que les droits d'usage, héréditaires tant que la terre est cultivée ; mais il ne peut la vendre, car tous les membres de son lignage et peut-être ceux d'autres lignages y ont aussi des droits; il ne peut non plus la réclamer, s'il l'a abandonnée pendant quelques années et qu'un autre l'a fait fructifier. Les pâturages (pap) sont toujours communs. Un homme peut partager sa portion de terre cultivable avec ses parents, son gendre ou le fils de sa sœur par exemple, et c'est par ce lien personnel qu'un lignage étranger s'implante d'abord sur une terre. Parfois, un lignage étranger se développe aux dépens d'un lignage dont les ancêtres lui ont autrefois accordé la permission de cultiver la terre, il acquiert une emprise sur cette terre et commence à y recenser ses propres lieux d'habitation et ses propres tombes. Ou bien, le lignage propriétaire de la terre s'accroît, et lorsqu'il désire agrandir son domaine, il s'aperçoit que la terre est cultivée par les descendants des étrangers qui la considèrent maintenant comme leur appartenant de droit. Il conteste alors leur occupation, mais comme il ne peut directement protester contre leur usage de la terre, ni simplement les évincer de leur territoire puisqu'ils le font fructifier, il empiète sur leurs cultures et essaie de les en chasser en exerçant une pression continuelle. Les étrangers ont la ressource d'en appeler aux cours européennes constituées, mais lorsqu'ils se savent faibles, ils ne s'en soucient pas, sachant que, même s'ils gagnent, ils devront continuer à vivre au milieu de leurs puissants voisins qu'ils ont poursuivis en justice. Ces situations ne peuvent trouver de modes de règlement dans les coutumes ancestrales, et lorsque des querelles de ce genre éclatent, l'issue dépend de divers 
facteurs : la puissance des lignages étrangers, leur richesse, leur influence sur les chefs et leur ruse vénale.

\section{VII}

\section{$\underline{\text { Retour à la table des matières }}$}

Le caractère superficiel de l'enquête à l'origine de cet article explique son manque de précision. Ceux qui étudient les Luo actuellement fourniront sans doute un compte rendu plus complet, plus juste et plus nuancé, de leur organisation sociale, Néanmoins, cette recherche atteint le but qu'elle s'assignait. à savoir, montrer que la structure politique luo, avec son cadre lignager, reproduit, pour l'essentiel, celle que j'ai décrite chez les Nuer. Il existe quelques différences dont j'espère bientôt traiter dans une étude comparative sur les lignages nilotiques, dans le cadre de travaux sur les systèmes lignagers, que les recherches, basées sur des données africaines plus vastes et sur des données mondiales, font lentement progresser. Cependant, il ne sera peut-être pas facile de déterminer si les différences sont significatives, tant qu'une étude approfondie des Luo n'aura pas été menée à bien. La seule ambition de cet article consiste à montrer la similitude de structure chez les Luo et les Nuer. Des recherches récentes ont prouvé que les Anuak et les Shilluk avaient une structure lignagère, combinée cependant avec des caractéristiques apicales, telles que chefferie et royauté. D'autre part, les Lango semblent posséder un système lignager semblable à celui des Luo, et constituer comme eux une société acéphale. Malheureusement, le rapport de Driberg ne nous permet pas de juger du degré de similitude existant entre les lignages luo et les lignages lango, ni la fonction politique du lignage lango. Il serait nécessaire d'effectuer des recherches complémentaires avant de classer, en fonction de cet indice, les Lango dans la série typologique des peuples nilotiques. 
La femme dans les sociétés primitives (1963)

\section{Chapitre 12 \\ "Le mariage coutumier chez les Luo du Kenya" · (1950)}

\section{$\underline{\text { Retour à la table des matières }}$}

Cet article s'intègre dans un bref compte rendu sur les Luo, et exploite une enquête effectuée en 1936 avec l'aide de la Fondation Leverhulme. Il constitue la suite de l'analyse des structures politiques luo ${ }^{1}$ (chap. 11).

A l'exception des renseignements fournis par le P. Hartmann ${ }^{2}$ et d'un récit plus complet de K. C. Shaw ${ }^{3}$, les études sur le mariage luo sont rares, et en certains cas erronées. Le récit de Shaw recoupe en grande partie le contenu de cet article, mais il est utile de posséder deux rapports indépendants, d'autant que les informations ont été recueillies à l'aide d'interprètes. Lorsque je me rendis au Kenya, je ne comptais pas travailler sur les Luo et n'avais donc pas lu l'article de Shaw. Nous sommes en désaccord sur un certain nombre de détails, ce qui ne signifie pas que l'un de nous ait tort, car, comme Shaw le fait remarquer, il existe des variantes dans les coutumes des différentes tribus du pays luo. Mes renseignements sur ce sujet particulier se rapportent surtout à la tribu alego du centre Kavirondo. Dans l'ensemble, je me suis inspiré du récit d'un pasteur de cette tribu, le pasteur Ezekiel, en omettant bien des détails.

Article paru dans Africa, 1950.

E. E. EVANS-PRITCHARD, Luo Tribes and Clans, Rhodes-Livingstone Journal, 1949.

2 H. HARTMANN, Sorne Customs of the Luwo (or Nilotic Kavirondo) living in South Kavirondo, Anthropos, 1928.

3 K. C. SHAW, Some Preliminary Notes on Luo Marriage Customs, J.E.A. et U.N.H.S., 1932. 


\section{$S I M B A$}

\section{$\underline{\text { Retour à la table des matières }}$}

Faire la cour et se marier sont deux actes intimement liés à la coutume du codo (rapports sexuels entre jeunes garçons et filles) dans la case des garçons célibataires (simba), la plus proche de l'entrée dans la concession luo. Les jeunes gens se concertent avec les jeunes filles pour qu'elles leur rendent visite le soir, et lorsqu'un garçon reçoit une de ses bien-aimées, les autres jeunes gens de la case vont dormir ailleurs. Le jeune garçon s'amuse avec la jeune fille entre les cuisses de laquelle il éjacule, puis ils dorment ensemble. Il ne doit pas la pénétrer pour éviter le déshonneur qui s'ensuivrait le jour de son mariage. Si une jeune fille vient de loin voir son ami, elle peut passer plusieurs nuits avec lui dans la simba. Une jeune fille peut avoir, en différents endroits, plusieurs amis auxquels elle rend visite, de temps à autre, à son gré. Pas plus que les jeunes gens ne se querellent entre eux à son sujet, son père et ses frères n'interviennent dans ses affaires de cœur. Elle peut continuer ainsi pendant les diverses phases préparatoires de son mariage, jusqu'à la cérémonie finale du riso. Une jeune fille ne se rend pas habituellement à la simba plus d'une ou deux fois par mois, m'a-t-on dit. Lors d'une de ses visites, le garçon loue les services d'un joueur de harpe pour distraire amants et amis qui chantent et dansent. Entre deux chants, les garçons, voire les filles, se lèvent, puis se glorifient de leurs qualités, du nombre de leurs amis et de leurs bien-aimées, de leur richesse, de leur famille et de leur lignage; lorsqu'ils ont terminé, ils lancent un pourboire au musicien, un shilling par exemple. Des concurrents redoublent alors de fanfaronnades et de prodigalité.

\section{POR}

Retour à la table des matières

Ce texte a trait au mariage luo tel qu'il se pratique habituellement. Il existe une autre forme de mariage, le mariage par enlèvement, por, jugé honteux et ridicule. Un garçon amène une jeune fille à la simba et la pénètre. Il la garde ensuite chez lui, où elle fait la cuisine, ce qui indubitablement signifie qu'elle se considère comme sa femme. Ou bien, un homme et sa bienaimée s'enfuient et vivent ensemble dans une tribu éloignée. Les parents de la jeune fille peuvent essayer de la faire revenir, mais si elle refuse, ils régularisent le mariage en acceptant quelques bestiaux en compensation. Cependant, l'amant n'en donne pas autant que si le mariage avait été négocié selon l'usage courant. Aujourd'hui, une jeune fille qui se marie selon les rites chrétiens est parfois appelée avec mépris dhako ma por parce qu'elle fait la cuisine chez son mari immédiatement après son mariage. 


\section{MEKO}

\section{$\underline{\text { Retour à la table des matières }}$}

Le terme meko désigne la forme normale du mariage. Au sens restreint, il s'applique à sa première phase, l'enlèvement de la fiancée et sa défloration devant témoins. Lorsqu'un jeune homme a obtenu le consentement de son père, il se met en quête d'une femme qui lui convienne. Il se peut qu'il ait déjà fixé son choix sur une jeune fille du pays, mais souvent il se rend, avec un ami, au domicile d'un parent éloigné, dans le voisinage duquel il trouvera, hors de sa parenté, de nombreuses jeunes filles. Quand il entend parler d'une charmante jeunette, il se rend chez elle et demeure dans la simba jusqu'à ce qu'elle vienne bavarder avec lui en compagnie des autres jeunes filles de la concession. Si elle lui plaît, il lui rend de nouveau visite, accompagné de deux amis, dont l'un, jagam ou jatelo, ami des deux familles, a pour mission de servir de négociateur entre elles. Le jeune homme demande à la jeune fille de l'épouser. Si elle refuse il est rare que le mariage ait lieu, même si les parents y sont favorables. Si elle accepte, elle prie le jeune homme d'en discuter avec ses parents. Lors des débats avec les parents, le prétendant s'assoit entre ses deux amis, de sorte que les habitants de la concession peuvent, en jetant un regard dans la simba, savoir immédiatement qui postule le mariage. Le prétendant laisse parler le jagam. Il suffit au père de la jeune fille de savoir l'entremetteur ami de la famille pour être assuré que le soupirant dispose d'assez de bétail pour payer une dot dont il n'est pas encore question à ce stade.

La phase suivante est celle de l'enlèvement de la fiancée. Les jeunes garçons de la maison du prétendant viennent, de bonne heure le matin, chez la jeune fille et attendent à l'extérieur du kraal. Le jagam pénètre dans la concession et va au dortoir des filles (suwindhi) que surveille une matrone (pim) à laquelle il réclame à grands cris la fiancée. Dès qu'il a promis un cadeau, la vieille femme ouvre la porte. Aussitôt, le jeune fille commence à s'agiter, elle se cramponne à la vieille femme, crie, se débat tandis que le jagam l'entraîne. Les jeunes gens du village accourent à l'aide de la jeune fille et un combat fictif s'engage. Il est rare qu'une jeune fille s'oppose à l'idée de se rendre chez son fiancé dont elle peut être la maîtresse depuis longtemps, mais elle se doit de résister aussi bruyamment que possible, pour montrer qu'il s'agit d'un véritable mariage (meko) et non d'un enlèvement (por). Dès qu'on ne peut plus l'entendre de sa concession, elle suit tranquillement les jeunes gens. Arrivée à la maison de son fiancé, elle va droit à la simba où les frères et les soeurs du jeune homme la reçoivent. Les sœurs la taquinent et lui assurent que leur maison est beaucoup plus agréable que celle qu'elle a quittée. Les deux fiancés se tiennent à distance, timides et silencieux en publie.

Après le départ de la fiancée, les jeunes filles de sa concession sortent en chantant. Leur groupe, appelé jodariya ou omower, arrive le soir chez le fiancé. C'est alors qu'a lieu la défloration de la fiancée, en présence de sa sœur (jadong), éventuellement accompagnée d'une autre jeune fille de sa famille, et d'un des frères mariés du jeune homme. La jadong étend une peau de bœuf sur le sol de la case et y entraîne les deux jeunes gens nus. Elle éteint le feu, et le mariage est consommé dans l'obscurité, mais sans orgasme. Les témoins raniment le feu, et à la lumière, examinent le membre de l'homme pour y déceler l'hymen (ring ruok). 
Quand la jadong le trouve, elle annonce la nouvelle aux jeunes filles qui chantent à l'extérieur. Celles-ci entrent alors et frappent le fiancé en disant : «Tu as tué notre soeur. » Il se relève promptement et sort. Les visiteuses chantent toute la nuit avec les garçons de la maison et la fiancée reste avec eux dans la simba. Si la fiancée n'est pas reconnue vierge, la honte paralyse les réjouissances. Cependant, cela ne change rien à la validité du rite, et si plus tard il y a divorce, les parents du garçon doivent donner une vache, dher duong nyako, la vache de l'honneur de la jeune fille, qu'elle ait été vierge ou non.

De bonne heure le lendemain, les jeunes filles repartent en chantant, avec l'hymen. Quand elles entrent dans la concession de la fiancée, tous les gens de la maison jettent des cendres vers son père et sa mère. Les jeunes filles très affamées pour n'avoir rien mangé chez le fiancé prennent alors un repas. Les parents du fiancé n'ont manifesté aucune sollicitude envers elles : «Ce sont des ennemies. » La fiancée reste chez le fiancé avec la jadong qui lui tient compagnie. Aucune dot n'a été payée et il n'en a pas encore été question.

Peu après la consommation du mariage, la famille du marié remet à la jeune épouse une ceinture (cieno) munie d'une sorte de queue de fibres, symbole de son nouveau statut. Elle lui offre en même temps une chèvre et un petit cache-sexe.

\section{DIERO}

$\underline{\text { Retour à la table des matières }}$

Un groupe important, jodiro, se rend - généralement le lendemain de la défloration, je crois - en chantant, du domicile de la mariée à celui du marié. Ce groupe peut compter plus de cent jeunes gens et jeunes filles. Les garçons portent des gourdins, des boucliers et des coiffures en plumes d'autruche. Ils s'arrêtent devant la concession et envoient le frère de la mariée la chercher. Quand elle arrive, son frère lui demande : «Qu'en penses-tu ? Es-tu d'accord pour que nous réclamions un taureau ? Désires-tu rester avec ton mari et devenir sa femme ? » La jadong sortie avec elle, et au courant de ses intentions, répond pour elle. Le frère transmet le consentement de la jeune femme à ses compagnons. Ils pénètrent alors dans le kraal et désignent un bœuf de leur choix. Il se peut que le père du marié se contente de leur promettre un animal, mais généralement il leur en donne un sur-le-champ, non pas cependant celui qu'ils ont choisi, car c'est le meilleur de son troupeau. Après discussion, ils acceptent celui qu'on leur offre et l'emmènent avec eux. Le père de la mariée le reçoit comme partie de la dot. Deux ou trois jours plus tard, les sœurs de la mariée lui rendent visite et lui apportent de la bouillie que leurs parents accompagnent souvent d'une chèvre qu'ils ont tuée et fait cuire. L'épouse et ses belles-sœurs consomment viande et bouillie. Les sœurs de la mariée rentrent le jour même si elles habitent auprès, ou le lendemain dans le cas contraire. Après cette visite, la mariée revient habituellement chez ses parents. 


\section{LUPO}

$\underline{\text { Retour à la table des matières }}$

Environ une semaine plus tard a lieu la cérémonie du lupo. La famille de la mariée tue un taureau, deux peut-être si elle est riche, quelquefois aussi une chèvre ou un mouton et quelques volailles. A l'exception d'une patte avant, de la tête et de la peau conservées pour les anciens de la maison, le corps de l'animal est transporté cru au domicile du mari, par les jeunes cousins de la mariée que suivront plus tard ses jeunes cousines, avec les intestins cuits, un peu de viande prélevée sur les côtes et de la bouillie. Les jeunes gens portent, dans la case de la mère du marié, la viande que l'on répartit traditionnellement entre les membres de la maisonnée. Après avoir déposé la viande, les cousins de la jeune femme lancent leurs gourdins sur les volailles jusqu'à ce qu'ils aient tué un coq qu'ils rôtissent sur un feu de bouse pour le manger. Comme mets d'accompagnement, ils demandent de la bouillie aux parents du mari, sans obtenir toujours satisfaction, et s'en retournent alors chez eux, croisant en chemin les jeunes filles qui apportent la viande cuite.

A quelques centaines de mètres de la maison du marié, les jeunes filles s'arrêtent et envoient l'une d'elles à la simba inviter la mariée à venir manger. Celle-ci demande à la messagère si les autres jeunes filles de la maison ont été averties, ce qui lui vaut la réponse : « Non, c'est à toi de rassembler tes gens. » Elle appartient désormais à la maison de son mari et non plus à celle de ses parents. La jeune femme appelle les jeunes filles de la maison en plaisantant : "Venez manger. Nous avons faim. On ne voit jamais de bonne viande dans cette maison. Maintenant que vous avez la fille d'un homme riche parmi vous, vous mangerez de la bonne viande. » La mariée sort de la concession avec sa fidèle accompagnatrice, la jadong, et avec les sœurs de son mari. Toutes s'assoient et consomment la viande. La mariée n'en absorbe qu'un morceau. Pendant ce temps, les visiteuses chantent dans le kraal où les autres jeunes filles les rejoignent une fois leur repas terminé, pour fixer le jour de la prochaine cérémonie du duoko, c'est-à-dire du retour de la mariée chez ses parents. Les visiteuses repartent ensuite chez elles.

\section{DUOKO}

\section{$\underline{\text { Retour à la table des matières }}$}

Au sud du golfe Kavirondo, on appelle cette cérémonie ter. Elle se déroule quelques jours après celle du lupo. Les parents du mari et leurs amis vont en groupe (joduoknyako ou joternyako) rendre visite aux parents de la mariée. Avant de partir, à l'entrée de la hutte de la mère du mari, les femmes enduisent de beurre l'épouse et la jadong, puis elles leur jettent du sésame. La sœur du mari apporte une petite branche qu'elle tend à son frère en lui ordonnant de battre sa femme ; il s'exécute. Puis elle la présente à la femme qui en frappe son mari. On 
se met en route, généralement en emmenant une chèvre. La sœur du mari ouvre la marche, suivie de la mariée et de sa jadong. Quand le cortège atteint le domicile de l'épouse, les femmes de la concession sortent et barrent le passage. Une vieille femme, de préférence la grand-mère de la mariée, transperce la partie inférieure du bec d'une volaille, y passe une corde qu'elle attache au cou de l'épouse. Celle-ci sectionne alors la partie inférieure du bec et jette la bête à terre. Les jeunes garçons de la maison la tuent et la mangent. Les visiteurs pénètrent alors dans le kraal et présentent leurs respects à la mère de la mariée. On passe la nuit à manger, à boire, à chanter, à flirter. Le matin, le chef de la concession, selon ses moyens, peut tuer un bœuf pour que les visiteurs festoient. Ceux-ci s'en retournent ensuite chez eux en emmenant l'épouse seule, sans sa jadong. Après avoir passé plusieurs jours avec son mari, la femme revient chez ses parents.

Jusqu'ici il n'a pas été question de bétail, mais après cette cérémonie, le mari ou son père commence à payer la dot, et les parents de la femme, pour accélérer le paiement, exercent sur lui une pression en encourageant leur fille à revenir fréquemment chez eux. Le mari ne peut ni l'empêcher de partir, ni la faire revenir, mais il a le droit d'envoyer ses frères s'en saisir et la ramener chez lui, hurlante et trépignante, s'il l'aperçoit à un rassemblement publie, à un marché par exemple. Le mari offre un bœuf en contrepartie. A nouveau la femme repart chez ses parents; pour la récupérer il lui faut donner un autre animal, etc., jusqu'à ce que les parents soient satisfaits et lui laissent leur fille pour de bon.

\section{POWO}

$\underline{\text { Retour à la table des matières }}$

Le mari commence à payer la dot après la cérémonie du duoko, en envoyant à ses beauxparents un jeune taureau appelé dher powo parce qu'avant de le leur expédier, le père du jeune homme frappe l'animal avec une branche de powo. Après ce versement, la mariée reste environ un mois avec ses parents, puis a lieu le duoko powo, le retour de la branche, quand l'épousée revient chez son mari en compagnie de sa jadong. Elle y demeure quelques jours, puis elle repart chez ses parents.

\section{RISO}

$\underline{\text { Retour à la table des matières }}$

Comme le mari souhaite ardemment posséder son propre foyer, et que les retours constants de sa femme chez ses parents ne le lui permettent pas, il fournit le bétail aussi vite qu'il le peut. Lorsqu'il a versé de six à douze têtes de bétail en paiement, il dépêche son jagam, l'ami qui a négocié le mariage, et ses frères, pour informer son beau-père qu'il a maintenant payé la dot et qu'il souhaite que s'accomplisse la cérémonie du riso. Il promet de donner plus tard le reste du bétail. Après cette cérémonie, sa femme demeurera avec lui en tant qu'épouse au plein sens légal et domestique du terme. Elle fera sa cuisine, et, en cas d'adultère de sa part dans la simba, son mari recevra une compensation. Le riso constitue l'acte d'engagement du mariage. Quand le jagam et les frères de l'époux exposent leur 
requête, les parents de l'épouse se moquent d'eux : «Que sont douze têtes de bétail (par exemple) ? Vous voulez rire ! Allez dire à votre frère de revenir chercher ses bœufs. » Le jagam refuse d'effectuer d'autres versements, aussi lui dit-on de retourner chez son ami pour lui enjoindre de remmener son bétail. Au su d'une telle réponse, le mari convoque ses parents et amis pour l'accompagner chez ses beaux-parents. Afin de se les concilier, ils emmènent une génisse ou un jeune taureau avec eux. Le mari affiche un air déprimé et prend une attitude pitoyable. Il ne s'égaie même pas quand ses beaux-parents lui envoient de la bière dans leur simba. Les visiteurs réclament l'épouse. Le père de celle-ci peut consentir à la laisser aller s'il reçoit l'assurance que le reste des bêtes sera prochainement livré, mais il se montre habituellement obstiné. «Allez, dit-il, me chercher cette vache. Vous voulez rire. Je ne veux pas garder ma fille ici. C'est vous qui fixez la date de son départ. Vous pouvez l'emmener aujourd'hui si vous voulez, mais il faut que vous ameniez le reste du bétail d'abord. » Le jagam et le père discutent. Tout le monde sait plus ou moins ce qui va se passer. Si le mari est vraiment pauvre, pour finir, le père de la jeune femme cède. Si, au contraire, c'est un riche éleveur, le jagam accepte de donner une bête supplémentaire. Il est rare qu'à ce stade un mariage soit rompu pour une question de bétail. En cas d'entêtement du père, les parents et amis du mari s'en vont, laissant l'infortuné à son chagrin dans la simba de son beau-père. Le marié refuse la nourriture que lui envoie sa belle-mère, et de temps en temps mange dans le voisinage avec des amis. Après plusieurs jours, son beau-père l'interpelle : «Tiens, tu es encore ici ? Que fais-tu ?» Le jeune homme répond : «Je veux mourir dans votre kraal. » Le beau-père s'exclame : «Tu ne peux pas faire cela. Sors d'ici ! » Mais comme il refuse de s'en aller sans sa femme, à la fin, le beau-père déclare : "Je suis fatigué de vous deux. Sors et emmène ta femme avec toi, mais veille à m'envoyer rapidement cette vache », puis il appelle sa fille et lui enjoint de partir avec son mari. Las de leur présence, il les fait sortir tous les deux de la concession.

La cérémonie du riso se déroule alors chez le mari où sont rassemblés tous ses parents et amis. C'est jour de liesse. L'épouse et un groupe de jeunes filles de sa maison se rendent en chantant au domicile du mari. A quelques centaines de mètres de la concession, l'épouse et deux de ses amies se cachent. Les autres arrivent ensemble, entrent dans le kraal et se dirigent vers la simba. Un des frères du mari demande le reste du groupe, c'est-à-dire l'épouse. La soeur aînée de celle-ci répond : «Je ne comprends pas ce que vous voulez dire. Personne ne manque. Nous sommes toutes ici.» On envoie quelques jeunes gens en reconnaissance aux alentours chercher la jeune femme. Ils avancent à pas feutrés, l'œil et l'oreille au guet. Dès qu'ils l'ont trouvée, ils l'amènent à l'entrée du kraal où elle apostrophe son mari : «Je ne veux pas entrer dans ta maison. Donne-moi ce que tu sais. » Il promet de le lui donner plus tard, dans la simba, mais elle refuse d'entrer sans rien recevoir en échange. Il lui offre alors un bracelet (manyonge en pays alego, onyonge au sud du pays luo). En arrivant à la simba, elle accepte de se pencher pour entrer, à condition de recevoir un autre cadeau qu'on lui accorde généralement. Cependant, il se peut qu'un frère un peu brutal du mari l'attrape par la peau du cou et la pousse à l'intérieur. Dans la case, elle se déclare très fatiguée et désireuse de se reposer. Un des hommes la force alors à s'asseoir avant qu'elle ne réclame quoi que ce soit.

Les compagnes de la jeune femme passent la nuit chez le mari, certaines peut-être avec des jeunes gens du pays, dans leur simba. Le lendemain matin, la famille du mari amène un taureau ou un bœuf, appelle la plus âgée des jeunes filles et lui demande d'accepter l'animal. Elle l'estime toujours trop petit, mais le prend en définitive lorsque le mari allègue sa 
pauvreté. Le jour suivant, les visiteuses remportent quelques morceaux de viande chez elles, mais elles en consomment la majeure partie le soir dans la simba, après que les parents du mari ont offert une parure à l'épouse pour avoir accepté d'entrer. Ils doivent lui octroyer un autre cadeau pour qu'elle veuille s'asseoir. Tous les parents du mari y vont de leur offrande. La mariée s'assoit les jambes repliées sous elle, et il faut lui donner un nouveau présent pour qu'elle les allonge. Veut-elle cracher, elle le fait moyennant un autre don. Elle remarque que la suie peut lui tomber du toit dans les yeux; pour compenser le risque, on lui offre quelque chose. La famille du mari lui apporte beaucoup d'autres présents dont chacun possède un nom : ra yanyi maro, une chèvre pour compenser d'avance les remarques insultantes que le mari pourra émettre sur sa belle-mère ; ra luor tawo, une chèvre, pour que la femme place respectueusement devant son mari sa bouillie quotidienne; gir bangala higa, une parure en perspective des brûlures que la femme pourra subir en faisant la cuisine ; gir ote, une parure en échange des courses qu'elle accomplira à la demande de son mari ; gir rigolo olawo, une parure en prévision du nettoyage que nécessiteront les crachats du mari sur le sol ; gir logo, un cadeau en paiement de l'eau que la femme apportera chaque jour à son mari pour qu'il se lave les mains ; gir teno wic, un présent pour permettre au mari de dormir sur le bras de sa femme ; gir tngo kom, un don pour que la femme apporte le tabouret de son mari ; diend then, une chèvre pour qu'elle puisse cuisiner chez lui, etc. Ces cadeaux sont offerts à la mariée par les parents paternels du marié (yuro), notamment par ses frères. On doit nommer chaque don en le lui présentant. La femme ne garde aucun de ces cadeaux; les jeunes filles qui l'ont accompagnée et ses autres cousines les emportent.

Le lendemain matin, le mari taquine ses belles-sœurs et leur demande où elles ont passé la nuit. Elles répondent : "Vous êtes méchants. Vous nous avez fait du mal. Vous êtes des ennemis, vous et votre femme. » Après le repas de midi, les jeunes filles partent en emportant les cadeaux du riso et les reliefs de viande. Au sud du golfe, les parents du mari envoient la viande chez sa femme avant la cérémonie, à l'exception de ce qui servira à nourrir les jeunes filles qui l'accompagneront.

\section{SEPO}

\section{Retour à la table des matières}

Après la cérémonie du riso, la femme reste avec son mari. Elle peut rendre visite à ses parents après deux ou trois mois de vie conjugale, mais elle ne retourne pas chez eux sans cesse, comme précédemment. De même, la famille de son mari ne peut plus s'en saisir lorsqu'elle se trouve dans un lieu publie, car elle est, non plus la fiancée, miaha, mais l'épouse, dhako. Quelques mois plus tard, se déroule la cérémonie finale du mariage, la cérémonie du sepo, nommée gango au sud du pays luo. Un jour, le mari demande à sa femme de lui préparer personnellement sa nourriture, plutôt que de cuisiner dans la case de sa bellemère comme elle le faisait jusqu'à présent tandis qu'elle vivait avec son mari dans la simba. Les jeunes frères du mari ont, entre-temps, construit une nouvelle simba. Le mari commence alors, habituellement après la naissance de son premier enfant, à bâtir une véritable case d'habitation, ot. La femme retourne chez ses parents pour leur annoncer la décision de son mari. Nul ne soulève d'objection. Le père du mari lui-même donne son consentement. 
Derechef, l'épouse se rend chez ses parents pour réunir les ustensiles de cuisine nécessaires à son nouveau foyer. On lui donne des marmites, des gourdes, une cuiller en bois, etc. Si ses parents sont riches, ils ajoutent un bœuf, s'ils sont pauvres, un mouton ou une chèvre, quelques volailles et de la farine. Seules quelques jeunes filles raccompagnent la jeune femme chez son mari pour l'aider. A leur arrivée, la femme du frère du mari apporte trois pierres pour servir de trépied et propose à la jeune mariée de lui apprendre à faire la cuisine. Elle commence à rassembler le nécessaire, et quand tout est prêt, la mariée fait cuire le repas et le sert à ses cousines venues l'accompagner. Celles-ci ont-parfois amené avec elles un mouton ou une chèvre pour profiter d'un mets carné.

\section{MILOHA}

$\underline{\text { Retour à la table des matières }}$

A travers les différentes cérémonies décrites, le mariage a été mené à bien. Si le prétendant n'avait pas offert une quantité suffisante de bétail, les parents de la jeune fille n'auraient pas laissé s'accomplir les rites. Il semble qu'il existe chez les Luo plusieurs termes pour désigner la dot : miloha, dho nyuombo et dho keny. Le nombre d'animaux verses en paiement de la dot varie suivant les circonstances du moment. Selon les renseignements fournis par les premiers auteurs (Johnston, Hobley, Northcote, Stam et Hartmann), il semble qu'avant la peste bovine qui décima le cheptel luo à la fin du siècle dernier, le montant de la dot se situait entre vingt et quarante têtes de bétail. Depuis, et jusqu'à une date récente, elle oscillait de deux ou trois à six ou sept. Le récit de Shaw suggère qu'en 1932 on considérait vingt bêtes comme une dot satisfaisante. Selon mes propres recherches de 1936, je crois qu'à cette époque on pouvait l'évaluer à quelque quinze ou vingt bêtes.

Le bétail se divise en deux parties, le dho keny et le dho i keny. Les animaux du dho keny, qui constituent la première partie de la dot, portent un nom spécial et sont réservés à certains parents de la fiancée. Ils représentent le minimum nécessaire pour que le mariage puisse être conclu. Ce sont : dher maro gi nyatheni, la vache et le veau destinés à la mère de la jeune fille dher wuoro gi nyatheni, la vache et le veau destinés au père dher aluni, la vache de l'arbalète, une génisse destinée au frère du père ; dher ciewo, la vache au lait frais ; thuon dhok, un taureau pour le père ; ruath pala, le veau du couteau - si un animal de la dot meurt chez les parents de la fiancée, sa chair est envoyée au fiancé qui doit remplacer la bête, afin que ses beaux-parents aient malgré tout de la viande à consommer ; ruath dier, le boeuf sacrifié lors de la cérémonie du diero, et le ruath ringo, le bœuf de la viande - cet animal est immolé par la famille du fiancé afin d'approvisionner la mère de la fiancée en viande - on le tue chez elle, mais il n'est pas de moment déterminé pour l'offrir. A l'exception de la vache, accompagnée parfois d'un veau, que reçoit l'oncle paternel, et du boeuf tué pour nourrir les visiteuses à la cérémonie du diero, tous les animaux reviennent aux parents de la fiancée. Les termes par lesquels on les désigne varient suivant les différentes régions du pays luo.

Je crois qu'au sud du golfe Kavirondo, on ne distingue pas entre les bêtes désignées par un nom spécifique et les bêtes sans dénomination particulière, mais en pays alego la distinction est très importante. Lorsqu'ils conduisent les bœufs qui portent un nom chez le père de la fiancée, le jagam et les frères du mari veillent à ne laisser aucun doute quant à la 
dénomination et à la destination des animaux. Ces précisions préviennent toute réclamation ultérieure. Le mari n'envoie qu'une ou deux têtes de bétail du dho keny à la fois, et il lui faut habituellement deux ans pour liquider sa dette. Il doit en outre fournir les bêtes du dho i keny dont le nombre ne sera déterminé qu'au cours des négociations entre la famille du mari et son beau-père. Cependant, il existe une moyenne conventionnelle : en 1936 elle variait entre cinq et sept têtes de bétail. C'est à propos des bêtes supplémentaires, une ou deux, qu'ont lieu d'interminables discussions. Certaines sont offertes peu après le dher powo (compris dans le dho i keny), mais jusqu'à ce qu'il ait versé la totalité du dho keny, le mari n'octroie des animaux supplémentaires que s'il ne peut faire autrement, sinon il risquerait de devoir en accorder davantage qu'il n'escomptait. En plus du gros bétail, la famille du mari donne entre cinq et dix chèvres. Il se peut que le mari ne puisse s'acquitter de son dû en quelques années ; si son beau-père est compréhensif, il ne l'obligera pas avec trop d'insistance à régler la dette en suspens. Le dernier animal peut n'être offert qu'au mariage de la fille de la femme.

Bien que le père de la fiancée reçoive l'ensemble de la dot, la plupart des animaux seront employés à l'acquisition de femmes pour ses fils ; peut-être en donnera-t-il un ou deux à ses frères. L'oncle maternel de la fiancée obtient généralement une génisse, dher nyakewo, la vache de la fille de la sœur, mais je ne pense pas qu'on la considère comme faisant partie de la dot, et elle ne concerne en rien le père de la fiancée. On l'offre à l'oncle maternel en échange de ses dons : bélier, poulets, éleusine, et de son hospitalité à l'égard du mari, car un bon oncle maternel invite chez lui son futur neveu. Légalement l'oncle maternel n'a aucun droit à la génisse. Les Luo disent que le ner n'est qu'un ora, c'est-à-dire l'oncle maternel n'est qu'un parent par alliance (du père de la fiancée).

Il semble que le dernier animal offert soit le ruath ringo, le boeuf de la viande auquel j'ai déjà fait allusion. On l'appelle aussi hedho. Parfois ce boeuf n'est livré que plusieurs années seulement après le rite final du mariage. Un matin de bonne heure, avant le réveil du village, les parents du mari le conduisent chez sa belle-mère. En outre, ils prennent la précaution de sectionner le tendon d'une des pattes de l'animal en l'amenant dans le kraal, pour que les jeunes gens ne puissent l'accaparer, car il s'agit d'un boeuf à abattre, destiné à la mère personnellement, et qui sera tué devant sa case et non pas d'un bœuf à ajouter au troupeau. En retour, les parents de la femme offrent généralement un mouton à la famille du mari qui en mange une partie sur place et réserve le reste pour les absents de la famille.

Il faut noter que la famille de la fiancée doit aussi offrir des cadeaux à la famille du fiancé et que ceux-ci sont également désignés sous le nom de miloha. Il s'agit, en certains cas, d'animaux tués au cours des rites du mariage, mais il semble que les parents de la femme doivent aussi accorder, à divers moments, une ou deux bêtes aux parents du mari. Ils donnent, m'a-t-on dit, au moins deux ou trois têtes de bétail en tout, jusqu'à cinq s'ils sont riches, y compris celles qui sont sacrifiées durant les cérémonies. Ce détail requiert plus ample information.

Tout ce qui est versé par la famille du mari à celle de la femme entre dans la dot, et si divorce il y avait, le mari pourrait demander la restitution du tout, augmenté de la progéniture des animaux de la dot. Cependant, les Luo considèrent le divorce comme un grand malheur, et si la rupture est due à la mauvaise conduite de la femme ou à sa disparition du domicile conjugal, ses parents font tout leur possible pour maintenir l'alliance qu'ils ont contractée avec la famille du mari, en remplaçant la promise par une de ses sœurs ou par une cousine. 
Au cas où la femme meurt, il en va de même (le retour de la dot étant regardé comme la solution ultime et la plus indésirable), ses parents essaient aussi de la remplacer par sa sœur ou une cousine. On désigne ce processus sous le nom de apongo dhuonga, le remplissage du panier. Le mari devra peut-être attendre plusieurs années avant d'obtenir sa nouvelle femme. Il lui faudra revivre avec elle toutes les cérémonies du mariage et payer la dot, moins importante cependant : quatre ou cinq bêtes peut-être. En cas de retour de la dot, pour chaque enfant que la femme a donné à son mari, la famille de l'épouse conserve deux ou trois têtes de bétail selon les circonstances et selon le sexe de l'enfant. Tout enfant reste avec son père. Je pense qu'aucune bête ne doit être rendue après la naissance du second enfant. Cependant, même si une femme meurt en laissant plusieurs enfants, les parents de la défunte essaient de marier le veuf avec une cousine de son ancienne épouse, pour maintenir l'alliance.

\section{LIEND}

$\underline{\text { Retour à la table des matières }}$

Lorsque le mariage a été totalement réalisé, c'est-à-dire lorsqu'est né un enfant, les Luo estiment que l'union ne devrait être brisée en aucun cas. Si la femme meurt, elle doit être remplacée par sa sœur. Le pendant de cette coutume du sororat est le lévirat : c'est-à-dire l'élevage d'une famille par un des frères du défunt, au nom de celui-ci. Un des frères du mort se charge habituellement de la veuve, cependant si elle exprime le désir de vivre avec un parent plus éloigné de son mari, ses vœux sont respectés. Quelquefois, elle est épousée par un fils de son mari, né d'une autre union. Comme on croit ces mariages inféconds, le fait est rare, et si on ne l'interdit pas, on ne l'encourage pas non plus. Une telle union ne peut se produire que lorsqu'il s'agit d'une jeune femme récemment mariée, et parfois en réponse au vœu exprimé par le défunt. Selon la coutume, les frères du mari sont les compagnons habituels des veuves et les tuteurs légitimes de leurs enfants. Il est courant qu'un Bantu, jamwa, ou un étranger au clan, jadak, passe ses nuits avec la veuve avant que le frère du défunt ne s'en charge ; au cas où l'esprit du mort chercherait à nuire, le Bantu ou l'étranger en serait alors la victime.

Il convient de préciser que le lévirat ne constitue pas un remariage. Aucune dot n'est versée, et si des enfants naissent de l'union, ils prennent le nom du mari défunt et non celui de son frère. Le mari défunt est leur pater. Son frère n'est que leur genitor. Si des filles viennent au monde, ce sont leurs frères, et non pas celui qui les a engendrées, qui reçoivent leurs dots. Le genitor ne se voit attribué que les deux animaux dus au frère du père, dont l'un porte alors le nom de «vache de la procréation » (dher nysolo ou dher oding). En outre, personne n'utilise le terme habituel kendo, se marier, pour désigner le mariage léviratique, mais on emploie les mots atero ou alago qui signifient prendre une femme pour vivre avec elle. La veuve est ci liend, la femme de la tombe, c'est-à-dire la femme du défunt. 


\section{$\boldsymbol{L U R}$}

$\underline{\text { Retour à la table des matières }}$

La coutume d'évitement est de pratique courante chez les Luo, elle fait partie d'une attitude générale de respect (lur) envers les parents de la femme, que le mari appelle kaorca (mes parents par alliance). Si un homme rencontre quelqu'un de sa belle-famille le long d'un sentier, il fait un détour, et s'il doit lui parler, il tourne le dos, notamment lorsqu'il s'adresse à sa belle-mère qui doit aussi tourner le dos. Même actuellement, alors que les hommes portent des vêtements, un beau-fils ne se rend pas chez sa belle-mère sans se recouvrir les parties génitales d'une bande de peau, Celui qui ne se préoccupe pas de ces normes est appelé wangatek, l'homme au regard indiscret, et il lui faut offrir un cadeau en compensation de sa négligence. Un mari doit respect à tous les membres du lignage dans lequel il est entré. Il peut regarder les jeunes en face, mais non les anciens. Il respecte également les ascendants de l'oncle maternel de sa femme, car ce sont aussi ses parents par alliance, orce. Les frères du mari ne doivent déférence qu'aux beaux-parents de leur frère et à ses cousins proches de la même génération.

Lorsqu'une femme a accouché de son premier enfant, les règles d'évitement se relâchent, et au fur et à mesure que les années passent et que le nombre des enfants augmente, les relations entre un homme et ses beaux-parents perdent de leur formalisme. Probablement après la naissance du premier enfant, a lieu une cérémonie qui permettra au mari, au lieu de rester dans la simba, d'entrer dans la case de sa belle-mère et d'y prendre son repas, lorsqu'il lui rendra visite. Les parents de la femme envoient le jagam chercher le mari, ses parents et ses amis, tous ceux que la femme appelle kacuoga, les proches de mon mari. Quand ils arrivent dans la case de la belle-mère, on place devant eux un grand plat de poisson, de la bière, du beurre fondu et la viande d'un bœuf tué et cuit pour l'occasion. Lorsqu'on les invite à se servir, le mari ne répond rien, mais ses frères déclarent ne manger que si on leur donne une chèvre. On les presse, ils refusent. On leur apporte donc une chèvre, on la leur montre, et le frère aîné du mari la prend. Ils mangent alors. La belle-mère et le beau-père offrent une pleine gourde de bière au mari après en avoir bu eux-mêmes une gorgée. Le mari boit et passe la gourde à son frère aîné. A son tour, le frère du mari présente une gourde pleine de bière au beau-père. Une fois le festin termine, les parents et amis du mari rentrent chez eux.

\section{CIENO}

\section{$\underline{\text { Retour à la table des matières }}$}

Nous avons noté qu'après la défloration, on offrait à la mariée un petit cache-sexe et une ceinture munie d'une «queue», cieno, laquelle est le signe de son nouveau statut matrimonial. J'aimerais en dire quelques mots en conclusion de cet article. Jusqu'à une date récente, filles et garçons sortaient nus, à l'exception d'un petit cache-sexe qui servait de 
parure dans les cérémonies publiques, et ce n'est qu'après la consommation du mariage que la femme portait une «queue ». Celle qu'on lui présente après sa défloration est empruntée à la femme du frère de son mari. Plus tard, on lui en offre une autre. Jusqu'aux cérémonies finales du mariage, la fiancée peut enlever son cieno chez ses parents, bien qu'elle doive le conserver chez son mari. Par la suite, elle ne doit jamais l'ôter ; même aujourd'hui, alors que les femmes s'habillent, elles portent aussi leur «queue ». Autrefois une femme mariée n'avait rien d'autre sur elle que son cieno. En fait, m'a-t-on dit, un homme pouvait divorcer si sa femme ne le gardait pas toujours. Les femmes ont deux cieno, l'un pour tous les jours, et l'autre plus joli pour les cérémonies. Une femme divorcée conserve son cieno si elle a eu un enfant de son mari, sinon elle l'enlève. Le premier acte d'une veuve en apprenant la mort de son mari est d'ôter son cieno et de le jeter sur le toit de sa case. 


\section{Chapitre 13}

\section{"Symbolique des noms de bœufs chez les Dinka Ngok" · (1934)}

\section{$\underline{\text { Retour à la table des matières }}$}

Les Nilotes désignent leur bétail de différentes manières, entre autres par sa couleur ou par référence à un animal, un oiseau, un reptile dont la couleur est mentalement associée à celle de telle ou telle vache. On obtient ainsi, par analogie de couleur, la vache crocodile, la vache aigle pêcheur, la vache léopard, etc. Les Dinka vont même plus loin et utilisent une double analogie dans la dénomination de leurs bœufs par des termes qui suggèrent soit une activité, soit un attribut de l'animal de couleur semblable à celle du bœuf en question. Lors de son initiation, le jeune garçon adopte un nouveau nom, celui du bœuf que lui offre son père. Le processus de transfert d'un nom de bœuf à un homme se présente donc comme suit :

a) Couleurs du bœuf ; b) Animal aux couleurs analogues à celles du bœuf ; c) Activité ou attribut associé à cet animal ; d) « Nom de bœuf » du jeune homme.

Je ne revendique pas cette découverte. Le Pr Seligman et sa femme écrivent: «Les exemples qui suivent, recueillis auprès de l'archidiacre Shaw, illustreront le cheminement de la pensée. Un jeune garçon propriétaire d'un bœuf appelé Manyang, de ma, mâle, et nyang,

Article paru dans B.S.O.S., 1934. 
crocodile, parce qu'on juge ce reptile plus ou moins tacheté, prend le nom Magor, gor désignant la mangouste tachetée. Quiconque possède un bœuf du nom de Majak, jak, pélican, peut adopter le nom d'Anoklek, nok, vomir, lek, un poisson, tandis que le possesseur d'un bœuf nommé Makwel, kwei, aigle pêcheur, prend le nom Akuemuk, c'est-à-dire « celui qui a les ailes raides », par analogie avec l'oiseau qui s'abat sur sa proie... Ainsi, le nom de tous les veaux gris comporte le mot lith, Melith, jeune taureau gris, de lith, faucon gris. Shaw remarque qu'on attribue aux vaches, mais non aux génisses, un nom qu'elles conservent leur vie durant; une génisse grise, Nalith, devient Gopdit, chasseur d'oiseaux, après avoir mis bas un veau marron clair, et une vache appelée Namer devient Pelawan, «celle qui dégage une odeur », par analogie de couleur avec le fruit parfumé lang » ${ }^{1}$.

Il semble que ces exemples aient été rassemblés parmi les Dinka Bor, sur la rive est du Nil. Je les complète ici par d'autres, recueillis près d'un garçon qui fut à mon service pendant une brève période de mon séjour en pays nuer, Biel du clan Fanai des Dinka Ngok qui vivent au sud de la rivière Sobat, près de son point de confluence avec la rivière Filus. Dans la liste ci-dessous figurent les équivalents en nuer, marqués de la lettre $\mathrm{N}$, car nous conversions dans cette langue.

1 - Mior ma ${ }^{2} \mathrm{ku}$ (N. thak mabor), bœuf blanc. Second nom, ruil pei (lune brillante) ; la nuit, son pelage reluit comme la lune.

2 - Mior ma jaak (N. thak ma jaak), boeuf au corps blanc, mais à la tête, au cou et à la croupe bruns ou fauve, évoquant le pélican (jaak, N. bong). Second nom, anoklek : le pélican vomit (nok) un poisson (lek) quand il a trop mangé ${ }^{3}$.

3 - Mior ma diing (N. thak ma diing), boeuf au dos (et peutêtre au ventre) blanc, mais aux flancs bruns (ou fauve). Second nom, deelgook ; l'oiseau marabout (deel, N. kil) rejette (gook) le poisson appelé diing (N. jwath) ${ }^{4}$. L'association poisson-boeuf provient-elle ici du son ou de la couleur? L'oiseau marabout rejette le poisson aux longues arêtes qui lui déchirent l'oesophage, lorsqu'il essaie de l'avaler. Pour cette raison, on aperçoit parfois l'oiseau marabout avec un poisson a moitié hors du bec. Le Dr Tucker suggère comme interprétation de deelgook, «le poisson est trop volumineux pour que l'oiseau marabout ne l'avale ».

4 - Mior ma thiang (N. thak ma thiang), bœuf rougeâtre teinté de mauve, couleur « châtaigne », qui rappelle celle du thiang. Tiang est un terme nilotique employé au Soudan et intégré au vocabulaire scientifique comme nom d'espèce. Second nom, akolbang : les tiang secouent (kol) l'encolure d'un côté sur l'autre (bang), lorsqu'en paissant, ils relèvent la tête.

5 - Mior ma yän (N. thak ma yän), boeuf fauve qui ressemble, par sa couleur, au campagnol jaune (yän). Second nom, ajotthiang : le lion de même couleur se saisit (jot) du tiang.

C. G. et B. Z. SELIGMAN, Pagan Tribes of the Nilotic Sudan, 1932, pp. 169-170.

Cette particule peut aussi se prononcer me ou mi.

Shaw propose une autre désignation possible, alang wer (en Dinka Bor), avaleur de la, rivière, qui se rapporte probablement au même oiseau.

4 Selon Stigand (A Nuer-English Vocabulary, 1932, p. 14), il s'agit d'un poisson qui ressemble au congre. 
6 - Mior ma lith (N. thak ma lith), bœuf gris (peut-être gris ardoise), dont la couleur rappelle celle du faucon (lith, N. nyalieth). Second nom, agoryai : le faucon disperse (gor) les petits moineaux lorsqu'il s'abat sur eux ; effrayés, ils s'envolent alors en criant yai.

7 - Mior ma lou (N. thak ma lou), boeuf gris souris ; sa couleur est voisine du plumage de l'outarde (lou). Second nom, buwut. L'association reste douteuse ; ce nom, m'a-t-on dit, évoque l'apparence lointaine de l'éléphant, semblable à un nuage, et dont la peau rappelle aussi celle de la souris ${ }^{1}$.

8 - Mior ma car (N. thak ma car), boeuf noir. Second nom, muth : nuit sans lune ou quartier de lune invisible. Par une telle nuit, on ne saurait distinguer un bœuf noir dans le kraal, mais seulement ceux tachetés de blanc.

9 - Mior ma lual (N. thak ma lual), boeuf brun rougeâtre, ressemblant à un serpent brun rougeâtre appelé lualdit ${ }^{2}$. Second nom, atukdier : l'hippopotame, de peau brune, pénètre en trombe (tuk) dans le barrage (dier) de pêche pratiquée sur la rivière.

10 - Mior ma cuor (N. thak ma cuor), boeuf moucheté dont le pelage ressemble au plumage du vautour (cuor). Second nom, awulei : la vautour s'abat (wu) sur l'animal (lei) tué dans la brousse.

11 - Mior ma nyääl (N. thak ma nyääl), boeuf blanc dont le museau, le dos et les flancs sont tachetés de marron, comme la peau du python (nyääl). Second nom, aderthok : le python s'enroule (der) autour de la chèvre (thok), la tue et l'avale.

12 - Mior ma ke (N. thak ma kär), boeuf au dos (et parfois au ventre) blanc, et aux flancs noirs (ou d'une quelconque autre couleur, à l'exception du marron, cf. no 3 ma diing). Cette appellation dériverait-elle de râle ${ }^{3}$ ? Second nom, thoukiit: à la saison des pluies, les grenouilles coassent toute la nuit dans les mares. Elles ont un maître de chant (kiit) dont la mort (thou) survient toujours à l'aube. La nuit suivante, un nouveau maître conduit le chœur des grenouilles. Comme le bœuf, la grenouille porte des taches.

13 - Mior ma wea (N. thak ma wea), bœuf aux taches couleur poivre. Second nom, apokwea : la nuit, le ciel (pok) est parsemé (wea) d'étoiles.

14 - Mior yom lou (N. thak ma kwe looka), bœuf au pelage gris souris (cf. no 7) et au museau blanc. Second nom, ajulgiet. L'origine en serait la suivante: malgré des bains fréquents, la couleur du pelage ne passe jamais.

15 - Mior ma kol (N. thak ma kul), bœuf brun ou fauve avec une tache blanche au milieu du flanc ou de la croupe, qui évoque le soleil (akol en dinka). Le mot nuer kul remonterait alors à une période où le nuer et le dinka ne se différenciaient pas, car le terme nuer actuel

\footnotetext{
Buwut pourrait aussi se traduire par «piétine le kraal », éventuellement applicable à l'éléphant.

Selon STIGAND (ibid., p. 21) il s'agit d'une sorte de cobra.

Dans son vocabulaire nuer, STIGAND (ibid., p. 16) note « kér, râle; râle brun au cou blanc qui vit sur la végétation à fleur d'eau ». Il n'existe aucun terme dinka pour désigner cet oiseau.
} 
pour soleil est cang ${ }^{1}$. Le second nom suggère cette association et vraisemblablement kul est une variante de kol. Second nom, aruilbeny : le chef (beny) est brillant. Il luit (ruil) comme le soleil. Mon informateur ajoutait que le soleil n'a peur de rien, il se manifeste dans toute sa splendeur.

16 - Mior ma nyang (N. thak ma nyang), bœuf tacheté comme le crocodile (nyang). Second nom, akomgel : le chasseur saisit sa proie loin des hautes herbes (kuth en nuer, akom en dinka) de la rive, comme le crocodile quand il bondit sur les animaux ou sur les hommes.

17 - Mior ma bil nyang (N. thak ma bil nyang), bœuf moucheté avec une tache blanche sur le flanc (cf. no 16). Second nom, gwicatiep : le crocodile (nyang) surveille (gwic) sur l'eau l'ombre (atiep) de l'homme qui se tient sur la rive.

18 - Mior ma ngok nyang (N. thak ma yil nyang), bœuf gris bleuté, avec des mouchetures. En nuer, ngok se rapporte au héron, ce qui laisse supposer une signification semblable en dinka. Qu'en est-il au juste ? Le seul mot yil que je connaisse en nuer veut dire graine de nénuphar. Second nom, kokdhim : quand on filtre (dhim) la bière, on heurte le fond du filtre tressé, ce qui produit le son «kok, kok, kok» qui s'entend de loin. La bière fermentée répandue sur le sol prend une couleur gris-bleu qui ressemble à celle du boeuf.

19 - Mior ma ngok (N. thak ma yil), bœuf gris bleuté (cf. no 18). Second nom, wacluau : wac signifie acide, et le nom se rapporte à une sorte d'orge germée de couleur gris-bleu (cf. no 18$)$.

20 - Mior ma cok rol (N. thak ma cok rol), bœuf à la patte de devant blanche et dont la tache s'étend sous le ventre. Cok désigne la patte. Second nom, acokjiuth : le bœuf ressemble à une jeune fille qui porte, à la jambe (cok), un anneau brillant (jiuth, N. ciek).

21 - Mior ma lek (N. thak ma bor lek), boeuf blanc dont les taches imitent la répartition et la couleur de celles de la girafe. Cette association avec la girafe trouve son explication dans le second nom, mais il est difficile de savoir pourquoi le boeuf ne porte pas le nom de cet animal. Il apparaît plus vraisemblable que ce nom dérive d'un certain poisson désigné par le mot lek (perche du Nil ?) en nuer et en dinka, sans que l'on sache s'il s'agit d'une analogie de couleur. Second nom, ajotdhu : la girafe se fait prendre (jot) dans un piège à roue hérissée de pointes (dhu $)^{2}$.

22 - Mior ma lek lou (N. thak ma lek looka), boeuf gris souris avec des taches semblables à celles du no 21. Le second nom, kombai, « boiteux », fait allusion aux hyènes qui courent à la manière des estropiés. L'association réside probablement dans l'analogie entre la fourrure tachetée de l'hyène et la peau du bœuf.

23 - Mior ma göök (N. thak ma luil). Bien que je ne sache définir la couleur de ce bœuf, je la suppose, d'après le nom dinka, apparentée à celle du babouin (göök). Second nom, aroktim : le babouin grimpe (rok) aux arbres (tim).

Dans le no 2, on peut noter un autre exemple de cette subsistance probable du mot ancien dans les noms de bétail, le terme nuer actuel pour pélican étant bong.

2 Selon Shaw, il existe, en Dinka Bor, un autre nom, adier kwec, couvert d'écailles, comme le poisson. 
24 - Mior ma bil (N. thak ma bil). Ce boeuf de n'importe quelle couleur (sauf brun, ce qui lui vaudrait d'être appelé ma kul en nuer et ma kol en dinka, cf. no 15) se distingue par une grande tache blanche au milieu des flancs. Second nom, acongbang : la grue couronnée, dont le plumage porte une tache blanche comme le pelage du boeuf, danse (cong) d'une patte sur l'autre (bang).

25 - Mior ma jok (N. thak ma jok), bœuf blanc ou d'une autre couleur (sauf brun fauve, qui lui vaudrait d'être appelé ma jak, cf. no 2), mais au cou, à la tête et à la croupe noirs. Second nom, midai : quand un vapeur du gouvernement passe, tous les habitants se précipitent vers la rive pour le regarder (dai). On suppose que les Dinka trouvent une ressemblance entre les couleurs du vapeur gouvernemental et celles du boeuf, mais, dans cet exemple, je reste sceptique sur l'association.

26 - Mior ma rial (N. thak ma rial), bœuf au corps blanc, avec la tête, la croupe et quelques taches sur les flancs et le dos, d'une couleur différente, noir habituellement. Second nom, babur: les Dinka remarquent une ressemblance entre le bœuf et le vapeur gouvernemental (babur). Variante du no 25. Mon informateur m'assura qu'il n'existait pas, dans la nature, de mouchetures similaires.

27 - Mior ma rol (N. thak ma rol), boeuf de n'importe quelle couleur avec une large bande blanche sur le cou, l'épaule et la patte de devant. Second nom, kacbeegh : calao (rialbegh en nuer), dont le plumage présente les mêmes teintes que la peau du boeuf.

28 - Mior ma yom (N. thak ma kwe) ${ }^{1}$, boeuf qui se distingue par un museau totalement ou partiellement blanc, contrastant avec la couleur du corps. Il ressemble au bel aigle pêcheur, au plumage noir, au cou et à la tête blancs (telle est au moins la signification du mot nuer kwe, usité aussi, selon toute vraisemblance, par les Dinka Bor). Second nom, gwangnhial : l'aigle pêcheur attend qu'un poisson pointe la tête à la surface de l'eau (gwang) en direction du ciel (nhial).

29 - Mior ma kwac (N. thak ma kwac), boeuf, quelle que soit sa couleur, tacheté à la manière du léopard (kwac). Second nom, adimkwac, dérivé du terme « moucheté » (dim).

Ces dérivations pourront paraître contestables, d'autant que je ne connais pas la langue dinka et que je me suis fié aux informations d'une seule personne. En outre, l'absence d'un dictionnaire dinka-anglais m'a empêché de vérifier la signification des mots. Néanmoins, je me suis aventuré à publier cet article, car, pour l'instant, les noms de bœufs chez les Nilotes sont encore mal connus, alors qu'ils présentent un grand intérêt sociologique. Ils illustrent l'usage du langage comme technique de relations économiques et montrent la formation des symboles qui se rapportent aux couleurs et à leur répartition. Ils contribuent au progrès des études comparatives sur les langues du groupe nilotique, car plusieurs mots nuer ne se comprennent étymologiquement que par référence aux termes dinka qui désignent les mêmes animaux. Tant que l'on ne connaîtra pas mieux les noms de bœufs des peuples nilotiques, notamment ceux employés par les Shilluk, on ne saurait estimer à sa juste valeur la

Selon Shaw, dans le dialecte Bor, ma kwe et ma yom désignent deux animaux au pelage différent, car le possesseur d'un bœuf ma yom adopte comme nom arei gor, « abîme les nénuphars ». 
signification exacte de ce phénomène. Une étude, accompagnée de dessins, sur les noms de bœufs chez les Nuer, paraîtra dans Sudan Notes and Records ${ }^{1}$.

1 Je suis au regret de dire que cette étude n'a pas encore été menée à bien (1964). Quelques-uns des noms les plus courants, accompagnés de dessins, figurent dans mon ouvrage sur Les Nuer (1940). 


\section{Chapitre 14}

\section{"Note sur la vengeance des esprits chez les Anuak du Soudan anglo-égyptien" * (1953)}

\section{$\underline{\text { Retour à la table des matières }}$}

Chez les Anuak, à l'instar de la plupart des peuples nilotiques, la magie (gyath) n'occupe qu'une place fort restreinte. Néanmoins, il existe chez eux un certain nombre d'experts que l'on pourrait dénommer magiciens. Ils portent le nom d'ajua et des branches fichées en terre signalent souvent leurs habitations. Le premier groupe se compose d'hommes ou de femmes indifféremment, qui, connus comme devins sous le nom d'ajuan wäri, exercent leur activité en jetant en l'air les lanières (wäri) ornées d'anneaux de cuivre, découpées dans le cuir de divers animaux, et en observant la façon dont elles retombent sur une peau de chèvre. Un second groupe, celui des ajuan awal, est formé en majorité de femmes dont le rôle est de guérir les malades en extrayant, après en avoir déterminé la position exacte, des objets introduits dans leurs corps par les sorcières. Ces femmes massent et enduisent d'huile le corps du patient, sur lequel elles placent une gourde contenant de l'eau. Elles entourent la gourde de

Article paru dans Man, 1953. 
leurs mains, la frappent et finalement sortent de l'eau les pièces à conviction : morceaux de peau, débris de coquilles et autres menus objets. Un troisième groupe d'ajua guérit les maladies dues à la vengeance des esprits, acieni. Cette croyance, comme le montre l'usage de l'une ou l'autre forme du mot cien, est commune à tous les peuples nilotiques sur lesquels nous possédons quelques informations. Mais, par comparaison, il semblerait, selon la brève analyse du colonel Bacon sur les Anuak, parue dans Sudan Notes and Records (1922), qu'elle joue un rôle plus important et plus évident dans ce peuple que dans tous les autres peuples nilotiques, car la maladie et la mort lui sont communément, peut-être même habituellement, imputées. Mon dessein se limite ici à noter le peu que je sais afin de faire progresser l'étude comparative de ce phénomène chez les peuples nilotiques.

La vengeance des esprits est une notion complexe qui n'implique pas uniquement la vengeance d'un homme mort à l'endroit d'une personne qui lui a fait du tort ou à l'endroit d'un parent de cette personne. Elle s'exprime aussi, au moins en certains cas, par une sorte de haine des esprits entre des familles et des groupes de parents, par exemple, A meurt et son esprit tue B, l'esprit de B tue alors un membre de la famille ou un parent de A, etc. Chacun venge sa propre mort. Les parents d'un défunt, m'a-t-on dit, découvrent par divination le nom de l'esprit qui a provoqué le décès ; ils attendent ensuite une vengeance. Quand un parent de l'esprit meurt, ils sont satisfaits, Les Anuak croient, semble-t-il, que les hommes meurent de cette façon et qu'ils se vengent ensuite de la même manière. Vraisemblablement, cette notion s'appliquerait aussi aux décès survenus au combat. En effet, en 1935, on m'a raconté que la mort récente Par maladie de nombreuses personnes, dans le village de Pocala, était due aux combats qui s'étaient déroulés l'année précédente, à la suite de différends entre nobles, pour la conquête du pouvoir, La plupart des participants à l'attaque du village avaient été tués, aussi leurs esprits se vengeaient-ils. Comme je l'ai expliqué dans Les Anuak (p. 73), au lieu d'enterrer un cadavre, on le place parfois sur un arbre, afin de hâter sa vengeance.

Quand une personne est atteinte d'acieni, on appelle immédiatement le guérisseur pour la soigner, le plus souvent une femme, appartenant au troisième groupe d'ajua mentionné cidessus. On craint qu'elle ne meure sans un traitement immédiat, car l'une des caractéristiques de la maladie due à l'acieni est sa rapidité fatale, par opposition à la lente progression de la maladie dont Jwok (Dieu, sous une forme ou une autre) est à l'origine. Cette maladie se caractérise par sa nature infectieuse, elle gagne les unes après les autres les personnes d'une même famille, sans contaminer celles qui lui sont étrangères, car elle a pour finalité la vengeance contre une famille particulière ou contre un groupe de parents. La guérisseuse opère en imposant les mains sur les différentes parties du corps du malade pour saisir l'acieni. Lorsqu'elle l'a pris, elle le frotte entre les paumes de ses mains; puis tandis qu'elle le tient prisonnier dans l'une d'elles, elle place cette main à plat sur le sommet de sa tête et la frappe avec son autre poing, ce qui a pour effet d'introduire l'acieni en son propre corps et de la rendre folle ; aussi se précipite-t-elle vers le leu où elle jette l'acieni, qui serait un moucheron, visible par l'ajua seulement.

Je fus témoin d'une «séance » de ce genre à Pocala. La concession d'Abula située près de l'endroit où j'avais dressé ma tente était touchée par l'acieni. Une jeune femme avec un bébé au sein était tombée raide sur le sol, et peu après, une autre jeune fille de la maison s'était affaissée, inanimée. La première se remit grâce aux soins d'une guérisseuse qui s'occupa ensuite de la deuxième malade. A ce moment, la première s'effondra de nouveau et les deux femmes gisaient sur le sol, les muscles raides, les bras en extension au-dessus de la tête. La 
guérisseuse appliqua le traitement décrit ci-dessus, Elle se pencha sur elles, leur suça le cou et les lèvres, puis sauta de côté avec, dans la bouche, l'acieni qu'elle rejeta dans sa main pour se l'enfoncer ensuite dans la tête. Entre-temps, les malades se mirent à geindre et manifestèrent d'autres signes d'angoisse. Peu après, elles se calmèrent, s'assirent tranquillement sur le soi cri tournant la tête d'un côté sur l'autre, l'air ahuri. Leurs parents s'adressaient à elles, leur répétant qui elles étaient, pour tenter de leur faire retrouver leur sens. Ils placèrent le bébé dans les bras de sa mère qu'ils encouragèrent à lui donner le sein.

Sur ces entrefaites, la guérisseuse, elle-même contaminée par l'acieni, dut se retirer rapidement. Nous entendîmes un craquement de branches brisées et nous la trouvâmes inanimée derrière une case. Entre-temps, une seconde guérisseuse arriva et essaya de soigner sa collègue. A ce moment, la première patiente sauta en l'air et retomba raide sur le dos. La seconde guérisseuse se précipita pour la soigner et fut bientôt aidée par sa collègue remise sur pied.

Quand je revins à Pocala quelques jours plus tard, les deux femmes étaient encore en traitement. Leurs parents consultaient un devin nuer de la région Gaajok, alors en visite chez les Anuak. Celui-ci utilisa les objets habituels du devin nuer, des coquilles de moules et une gourde. Il déclara que les jeunes femmes allaient mieux, qu'elles ne mourraient pas, et il demanda à Abula de sacrifier une chèvre. Quand Abula répondit qu'il n'avait pas de chèvre, le devin nuer lui conseilla de soigner les malades à la manière habituelle chez les Anuak, Cette nuit-là, près de la concession d'Abula, de nombreux habitants du village se réunirent et formèrent un cercle d'environ deux cents personnes. Ils frappaient des mains, tandis que les deux guérisseuses se relayaient à l'intérieur du cercle et le contournaient en dansant et en secouant des gourdes de graines. Au bout d'un certain temps, elles se précipitèrent vers les malades, arrachèrent l'acieni de leurs corps et coururent le jeter sur les braises d'un grand feu. Une demi-heure plus tard, à force de danser, les deux guérisseuses parvinrent à un tel état de frénésie qu'un homme dut les tenir fermement par la taille, tandis qu'elles dansaient autour du cercle et saisissaient l'acieni sur le front de plusieurs spectateurs. Parfois, elles attrapaient, par la tête, certains membres de l'auditoire et les entraînaient vers le feu. Quand elles jetaient l'acieni dans le feu, leurs gardes du corps devaient les retenir, de crainte qu'elles ne se précipitent dans les flammes; quelquefois, ils les portaient vers le feu et leur tenaient la tête en bas pour qu'elles puissent déposer l'acieni. Plus avant dans la nuit, les chants, les danses, les battements de mains devinrent plus intenses ; les hommes de l'assistance se levèrent. Les guérisseuses se déchaînèrent tant et si bien que leurs gardes du corps renoncèrent à se charger d'elles, sauf lorsqu'elles repartaient à la poursuite de l'acieni. L'excitation atteignait son comble, de temps à autre, quand l'une des guérisseuses se précipitant vers le feu en éparpillait les braises ou brandissait un tison enflammé au milieu des spectateurs qui fuyaient pour se protéger, jusqu'à ce que certains aient réussi à lui attacher les bras derrière le dos. A plusieurs reprises, elles tombèrent prostrées sur le sol. La «séance 7) se prolongea jusque vers les minuit.

Fin du texte 